\title{
A Behavioral Theory of the Merger Dynamics of the post-merger integration process
}

\section{Terrill L. Frantz}

May 2014

CMU-ISR-14-104

Institute for Software Research (ISR)

School of Computer Science (SCS)

Carnegie Mellon University (CMU)

Pittsburgh, PA USA

PhD Thesis Committee:

Chair: Dr. Kathleen M. Carley, PhD (SCS, CMU)

Dr. Linda Argote, PhD (Tepper School of Business, CMU)

Dr. James D. Herbsleb, PhD (SCS, CMU)

Dr. Richard M. Burton, PhD (Fuqua School of Business, Duke Univ.)

Submitted in partial fulfillment of the requirements

for the degree of Doctor of Philosophy

\begin{abstract}
This work is supported in part by the Air Force Office of Sponsored Research (AFOSR) (FA9550-11-1-0179) for multi-level modeling, the Office of Naval Research (ONR) (MMT N00014-06-1-0104) for dynamics, and the National Science Foundation IGERT in CASOS (NSF) (DGE 997276). Additional support came from Carnegie Mellon University's Center for Computational Analysis of Social and Organizational Systems. The views and conclusions contained in this document are those of the authors and should not be interpreted as representing the official policies, either expressed or implied, of the Air Force Office of Sponsored Research, the Office of Naval Research, the National Science Foundation, or the U.S. government.
\end{abstract}


Keywords: organization behavior, post merger integration, organization learning, organization performance, organizational culture, computer simulation, computational organization theory 


\section{ABSTRACT}

Numerous organizational mergers fail because the post-merger integration process fails: this research responds by investigating the concealed relationship of the known, premerger organizational characteristics, on the unknown, post-merger consequences. Various structural and behavioral characteristics of the merging organizations are studied to measure their association with two decisive outcomes: the integration of the distinctive cultures and the combined organization's performance. Considered are varied antecedent organizational characteristics, which are believed to have an unknown effect on the organizational communication and learning process, and therefore have impact-also to some unknown degree - on post-merger outcomes. This study employs computational organization theory (COT) techniques, such as agent-based modeling and computer simulation, to experimentally manipulate these organizational characteristics in a series of controlled experiments - all conducted within a virtual laboratory. Precise quantitative linear models, derived from the simulated data, are constructed that involve characteristics, such as: (a) organization- and team-level complexity, (b) individuals' predisposition for seeking and sharing information, (c) individual's task focus, (d) the structure of work teams, (e) task-specialization of the workforce, and (f) the level of infrastructure support provided by the organization. This study is comprised of three distinct sub-studies that each report on separate, though closely related, experiments, which are then combined and integrated into a unifying and comprehensive Behavioral Theory of the Merger. Broadly, the studies find and the theory puts forth that there is a higher consequence arising from pre-existing structural characteristics, over those that are cultural. Results also support the common expectation that organizational complexity is a powerfully influential characteristic on integration and performance; there is clear evidence that of the numerous characteristics studied, complexity is the most predominate pre-existing feature associated with the organization's performance and cultural integration- note that it is correlated negatively. The extent to which the organization provides infrastructure support is also quite pronounced; as expected, it has a positive correlation with both of the post-merger outcomes. Counterintuitively, individuals' willingness to share information has little association with the performance and integration of the organization. Many more meticulous 
findings of this sort are reported and analyzed, leading to a cohesive "A Behavioral Theory of the Merger", which is thus formally put forth. An adjunct of this study is the development of a comprehensive, computational model of post-merger integration dynamics. This mathematical model, and the contemporary methods utilized in this research, paves a way for scholars to study integration dynamics at granularity impractical using traditional organization-research methods. Furthermore, the findings provide qualitative guidance to support managers engaged in the practice of post-merger integration. By considering the theory and using these mathematical models, such managers can ground and inform their actual post-merger integration strategies according to these tools, which are uniquely informed by scientific study and can be tailored to specific real-world circumstances. 


\section{Dedication}

This thesis, my career, and my life are dedicated to the very lovely Darin and Nikita the finest and most supportive family in the world - which also includes Mom and Dad who taught me well by their supreme example. Of course, this body of research is dedicated to Kathleen Carley, who gave me the unbelievable opportunity to insert myself into the most progressive research community and university that exists - world-class indeed! 



\section{Acknowledgments}

Goodness, I cannot add yet another 50 pages to this thesis manuscript, so these eight sets of acknowledgments will be short and simple. First, I must acknowledge you (the unknown reader) for being brave so as to be taking a look at this manuscript- thanks! Second, third, and fourth, Dr. Kathleen Carley, well.... just can't put it all into words, frankly; instead, I'll just promise you that I will indeed publish in an "A" journal someday in my career. Fifth, I owe my world-class thesis committee, Drs. Linda Argote, Richard Burton, Kathleen Carley (again), and James Herbsleb: well, I promised you all a world-class thesis, so I am thankful everyone just "settled" along with me; but, thanks to you, I'll live to research another day...you're certainly my scholar-role models! Sixth, the awesome gang - past and current, near and far - at the Center for Computational Analysis of Social and Organizational Systems (CASOS) and at the broader, School of Computer Science at Carnegie Mellon University — is it quite simple: all are just awesome people with incredible minds! Seventh, as many of us aim to influence unmet, future scholars, thank you to Richard Cyert, James March, and Herbert Simon; you've done just that. Lastly, "Hey Connie [Herold]...it's finally done!"

Okay...now that this manuscript is done, I'm now on to working on doctoral thesis \#3 - Not! 



\section{TABLE OF CONTENTS}

TABLE OF CONTENTS

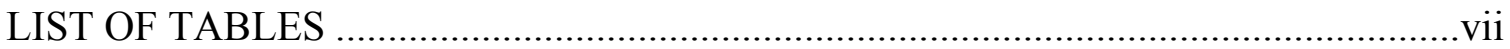

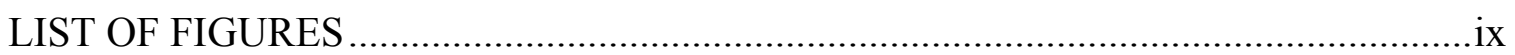

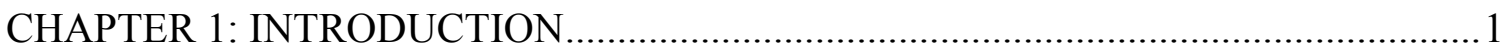

1.1 Overview …………

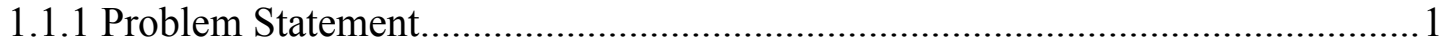

1.1.2 Phenomenological Exploration …………………..........................................

1.1.3 Methodological Matters...............................................................................

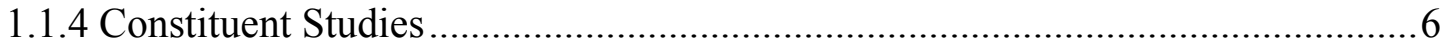

1.1.5 Outcomes: Scholarly \& Practice …………………....................................

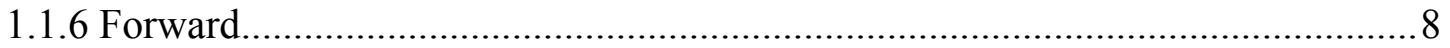

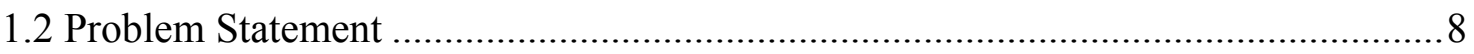

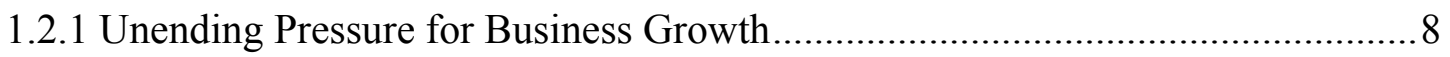

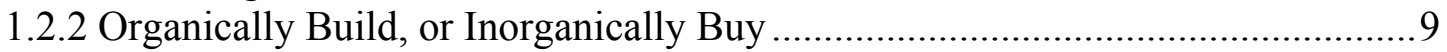

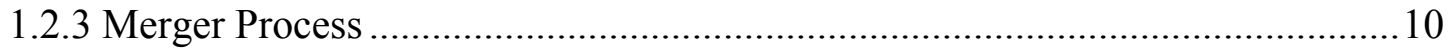

1.2.4 Chronic Failure of Mergers ........................................................................ 10

1.2.5 Forecasting Post-Merger Outcomes: A Quagmire …………………………......12

1.2.6 Post-Merger Dynamics: Little Understood .......................................................12

1.2.7 Overarching Research Question ..................................................................

1.2.8 Post-Merger: Uncertainty ……………………………................................ 14

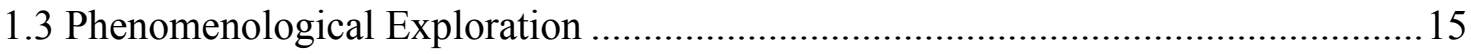

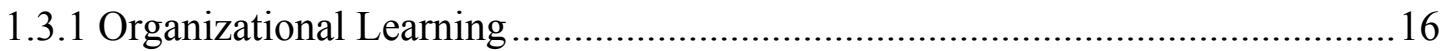

1.3.2 Post-Merger Integration Outcomes ................................................................ 18

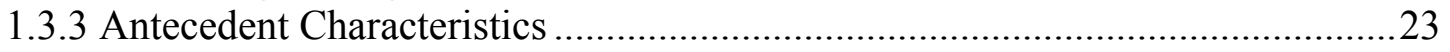

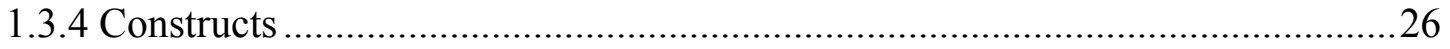

1.4 Methodological Matters ................................................................................

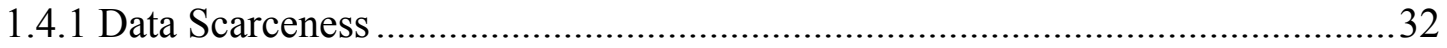

1.4.2 Computer Simulation \& Computational Models..................................................33

1.4.3 Controlled Experiments....................................................................................

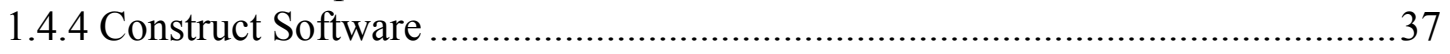




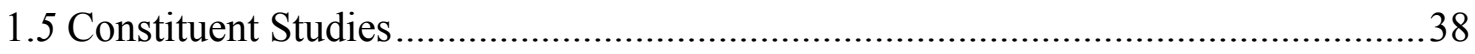

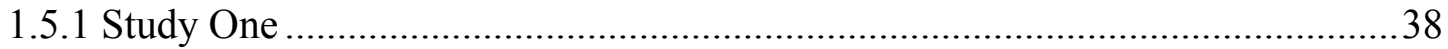

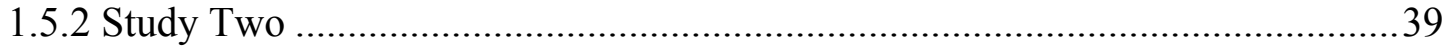

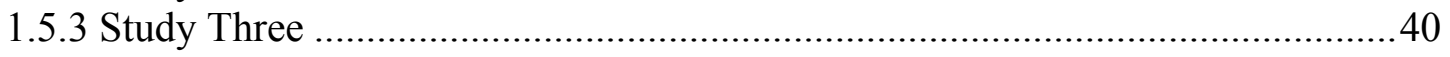

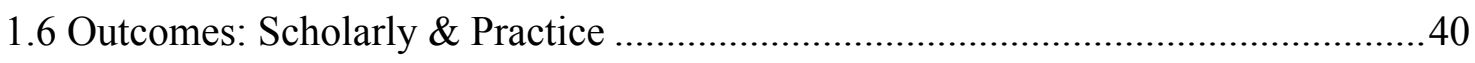

1.6.1 For Scholars: A Behavioral Theory of the Merger.............................................41

1.6.2 For Practitioners: A Forecasting Tool ..............................................................4

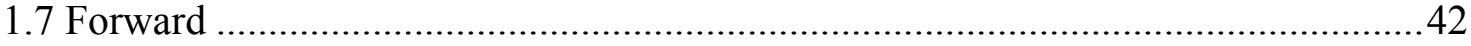

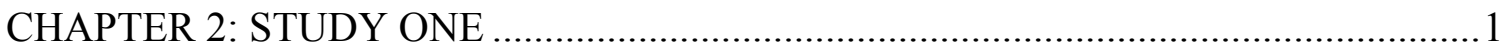

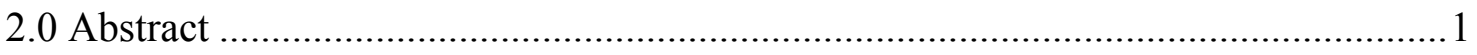

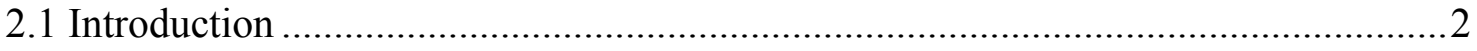

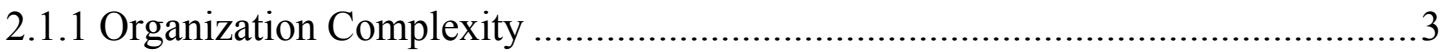

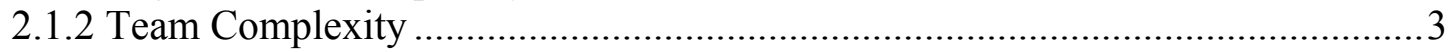

2.1.3 Infrastructure Support...............................................................................

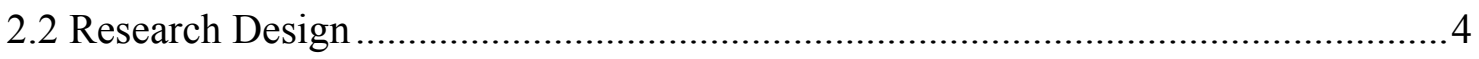

2.2.1 Simulated Case Scenarios.............................................................................

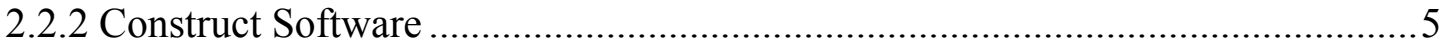

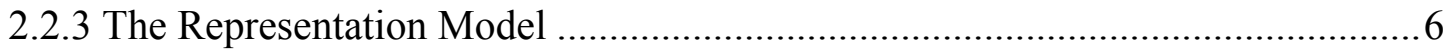

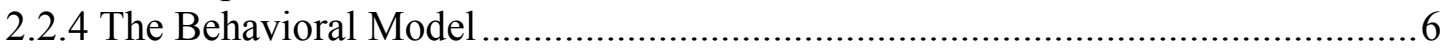

2.2.5 The Merger Transaction Scenario .................................................................

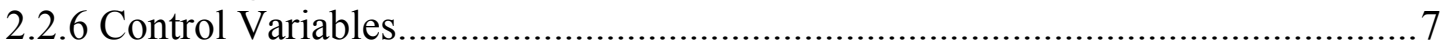

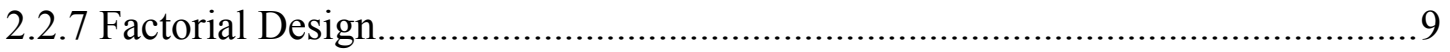

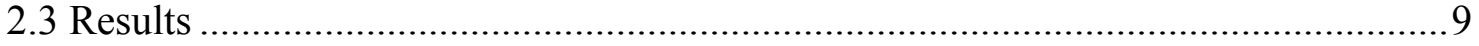

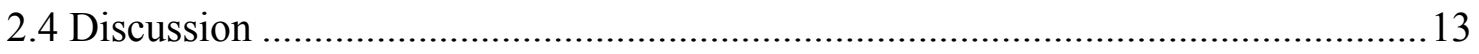

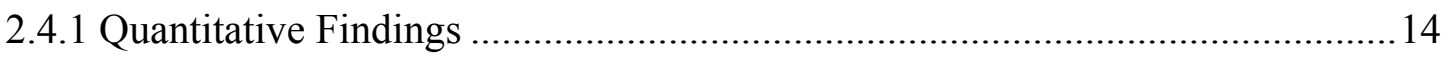

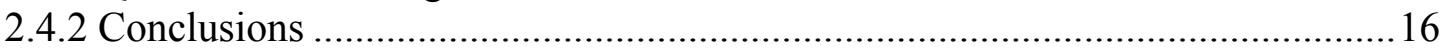

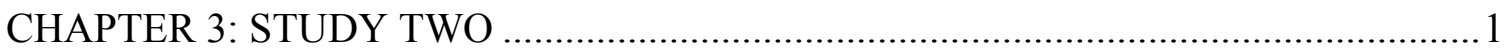

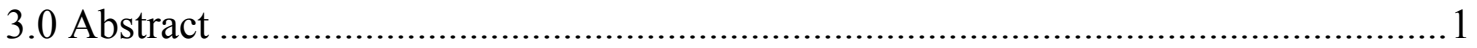

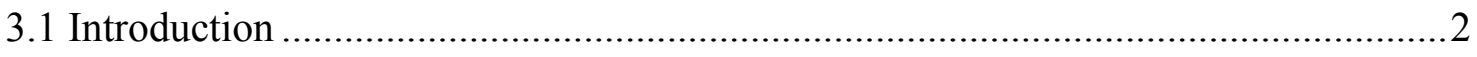

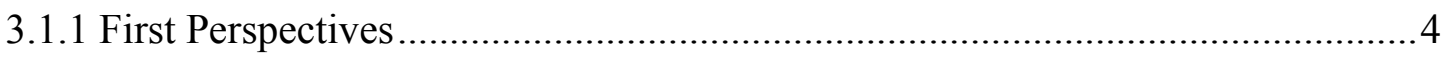

3.1.2 Research Questions \& Propositions .................................................................. 


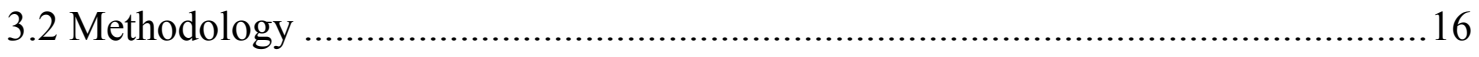

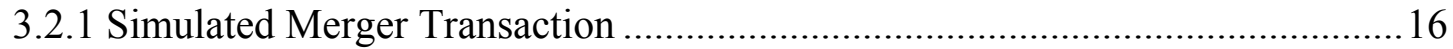

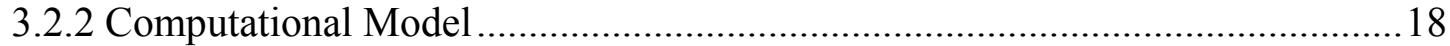

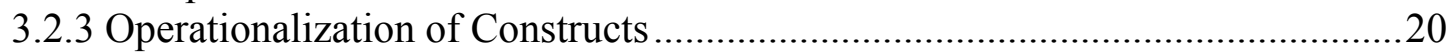

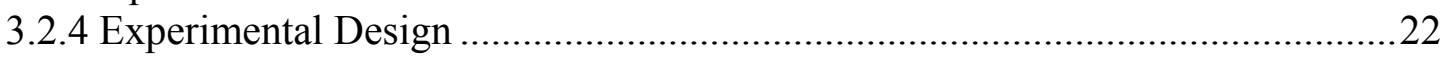

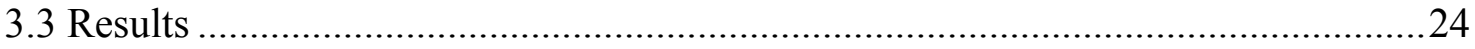

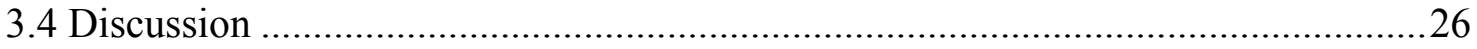

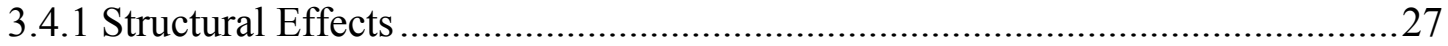

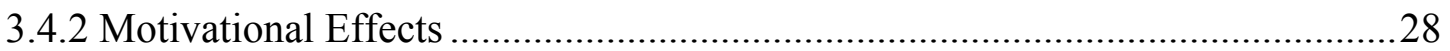

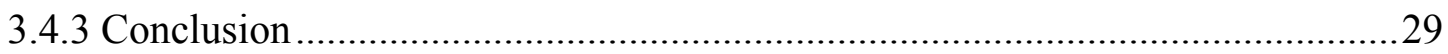

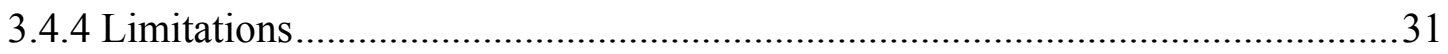

3.4.5 Executive Summary ....................................................................................

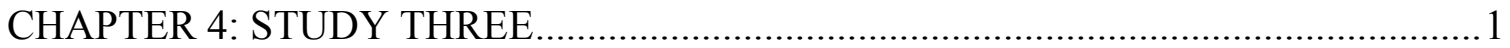

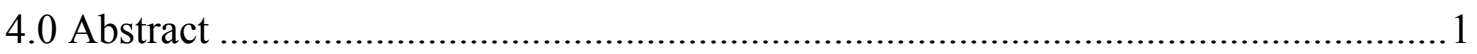

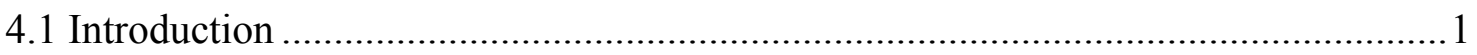

4.1.1 First Perspectives ....................................................................................

4.1.2 Research Questions .................................................................................

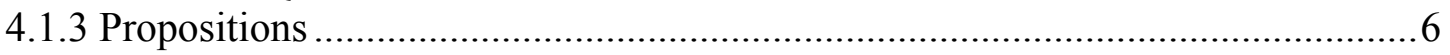

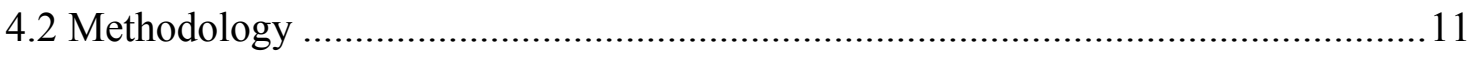

4.2.1 Simulated Case Scenario .............................................................................

4.2.2 The Computational Model..........................................................................12

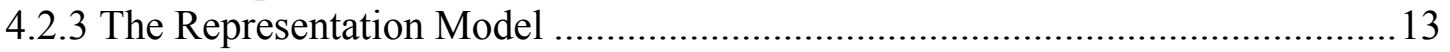

4.2.4 The Behavioral Model ..............................................................................13

4.2.5 The Merger Transaction Scenario .................................................................. 14

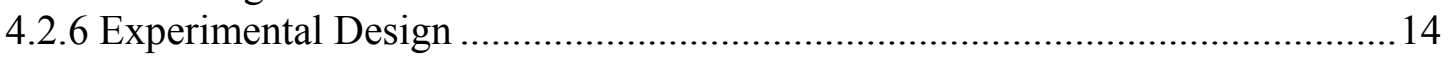

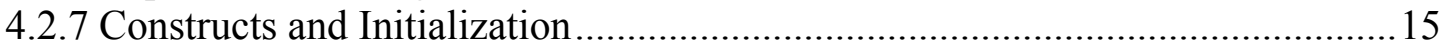

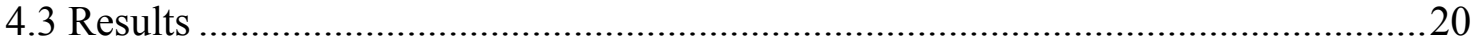

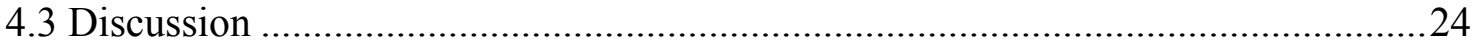

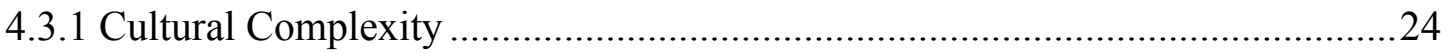

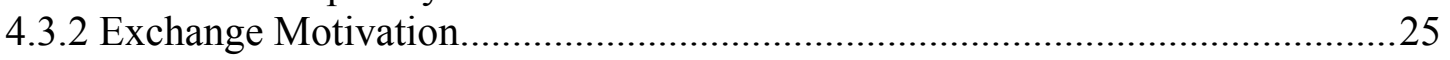

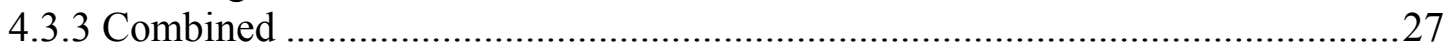

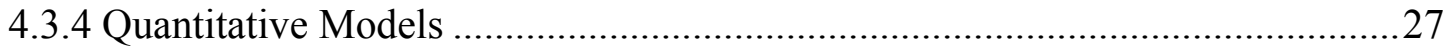

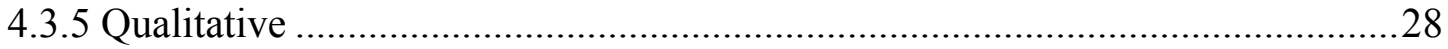

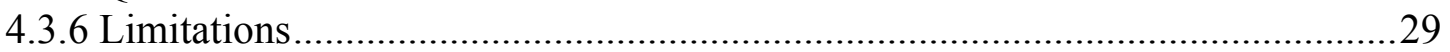




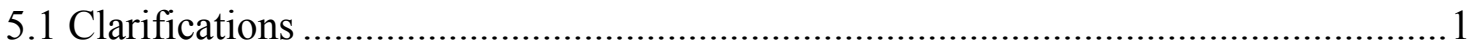

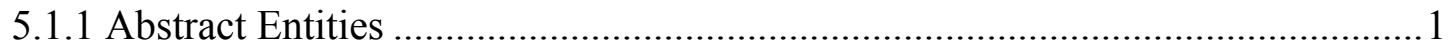

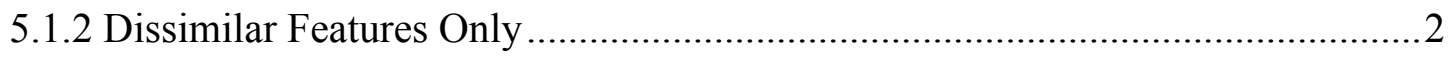

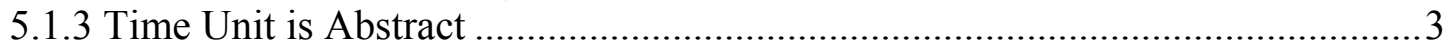

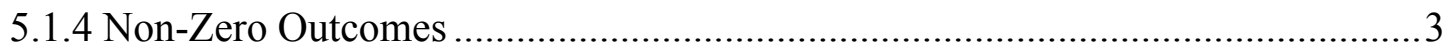

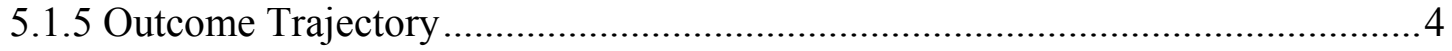

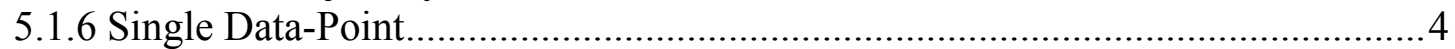

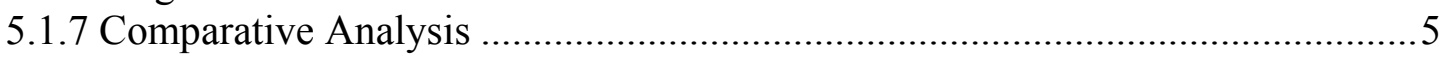

5.2 Models From Constituent Studies ...................................................................... 5

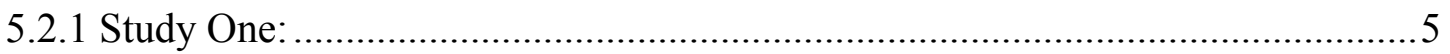

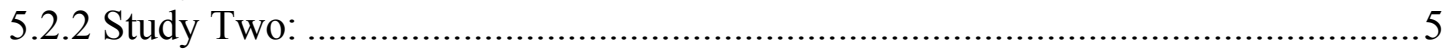

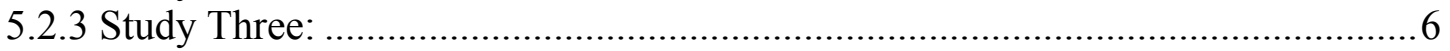

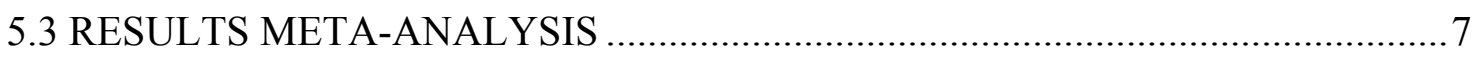

5.3.1 Interaction Effects ............................................................................. 7

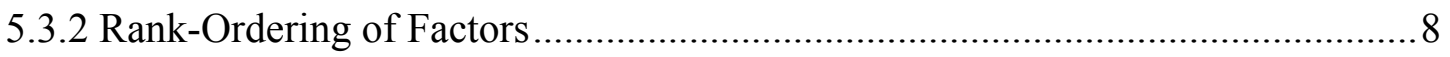

5.3.2 Structural Factors Outweigh Cultural........................................................... 9

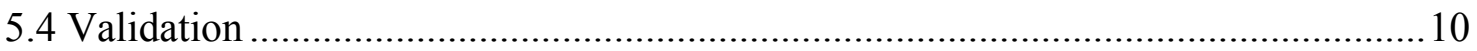

CHAPTER 6: QUALITATIVE FINDINGS ........................................................... 1

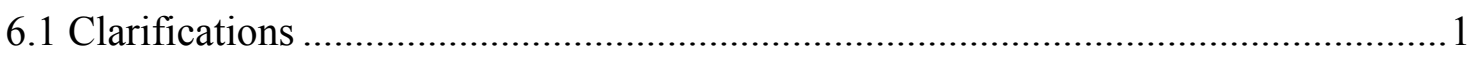

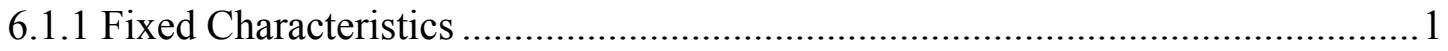

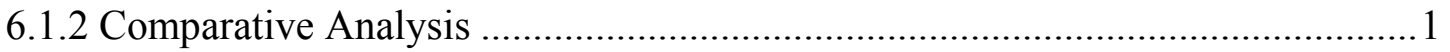

6.2 Synopsis of Constituent Studies ................................................................ 2

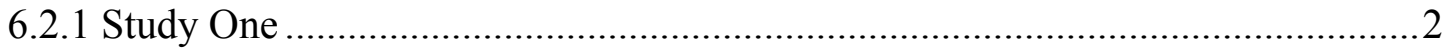

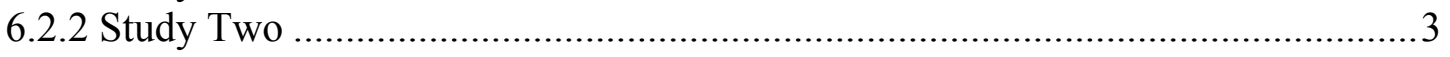

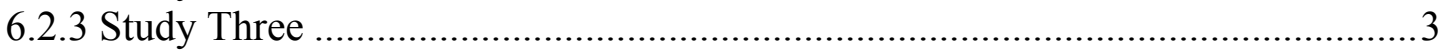

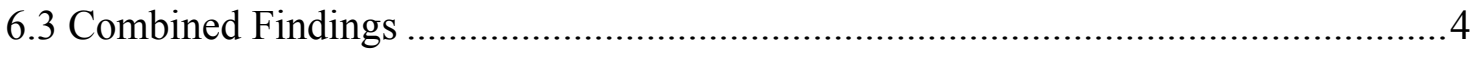

6.3.1 Rank-Ordering of Factors .......................................................................... 4

6.3.2 Structural Factors Outweigh the Cultural ..................................................... 5

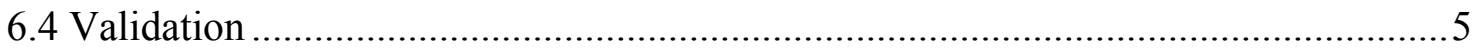

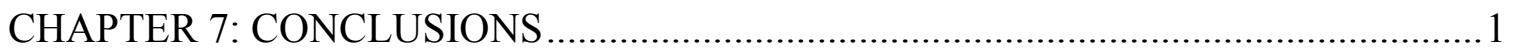


7.1 A Behavioral Theory of the Merger ........................................................... 1

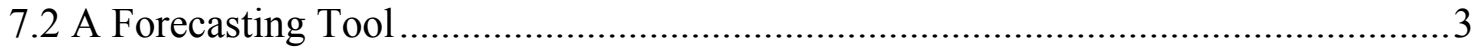

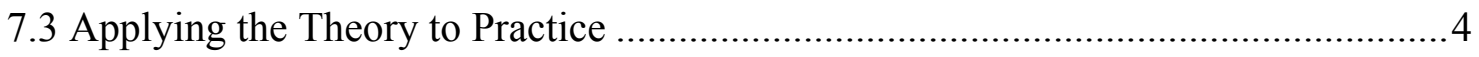

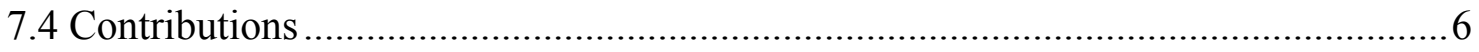

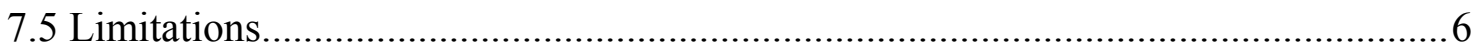

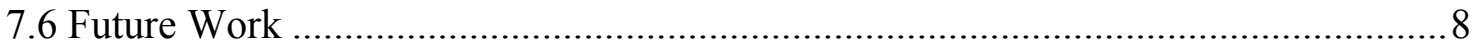

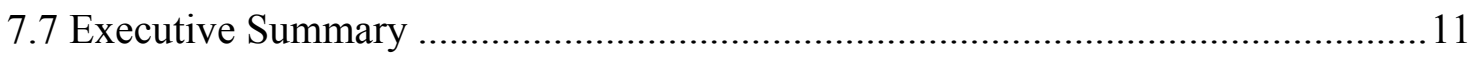

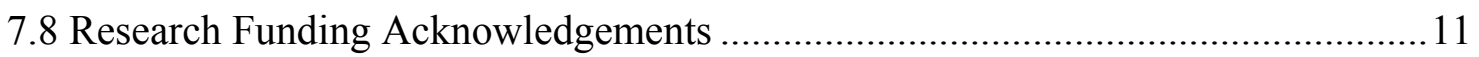

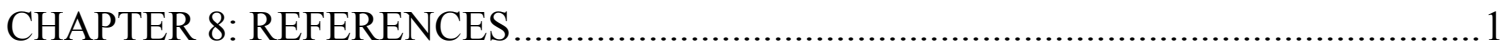

APPENDIX A: SYNOPSIS OF COMPUTATIONAL MODELS ................................. 1

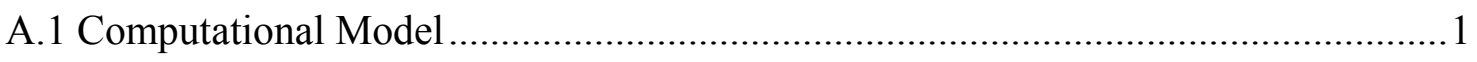

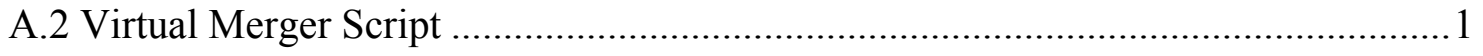

A.3 Representation Model............................................................................. 2

A.4 Agent Behavioral Model ...................................................................... 3

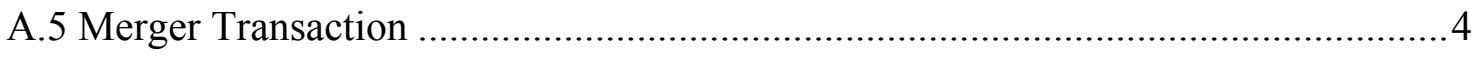

APPENDIX B: FIRST PERSPECTIVES OF THE ORGANIZATIONAL MODELS ...... 1

B.1 Organization as Information Processing Actors ............................................. 1

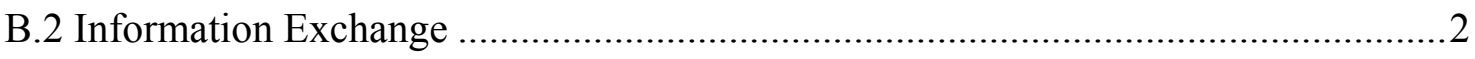

B.3 Factors affecting the Transfer of Knowledge ................................................. 4

B.4 Organizational Knowledge ................................................................. 4

B.5 Uniqueness of Organizational Knowledge .................................................

B.6 Shared Knowledge is Shared Culture ......................................................... 6 
B.7 Task Knowledge is Task Performance

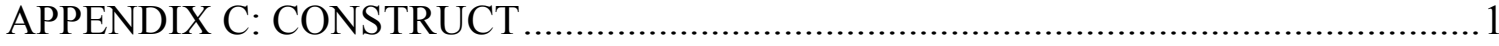

C.1 The Organizational Meta-network Representation Model .....................................2

C.2 The Organizational Actor as Behavioral Agent............................................... 3

C.3 The Organizational Actor as an Information Processor .......................................4

C.4 The Organizational Actor as an Adaptive Agent............................................... 4

C.5 The Organizational Actor Situated in the Organizational Meta-network.................6

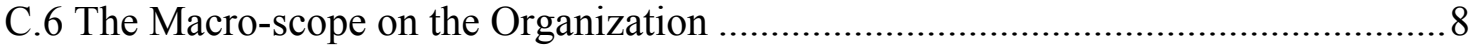

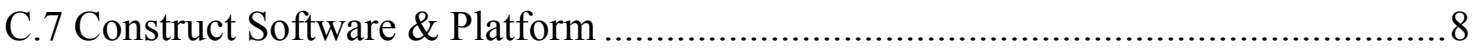

APPENDIX D: CONSTRUCT SOFTWARE \& CONFIGURATION ............................. 1 


\section{LIST OF TABLES}

Table1-1. All-inclusive Listing of Constructs Involved ............................................. 1-27

Table 2.1. Control Variables...................................................................................... 3-8

Table 2-2. Relationship between pre-existing factors and Knowledge Transfer:

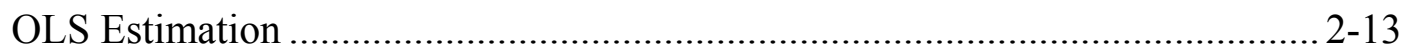

Table 3-1. Initial settings of control variables ......................................................... 3-22

Table 3-2. Descriptive statistics for response variable, Percentage of Task Knowledge (PTK) at time period 999; $\mathrm{n}=9,720$ each experiment .................................. 3-24

Table 3-3. Regression results (Ordinary Least Squares) .............................................. 3-24

Table 4-1. Summary of Propositions (Proposition id \& expected directional influence)....... 4-6

Table 4-2. Glossary of Constructs ............................................................................... 4-15

Table 4-3. Parameter Values of Control Variables ....................................................... 4-19

Table 4-4. Descriptive statistics for response variables, $n=9,720$ each experiment ........... 4-20

Table 4-5. OLS Regression Results ............................................................................. 4-20

Table 5-1. Rank order of Beta coefficients (standardized) for independent factors, gathered from all experiments (Absolute value; ordered greatest to least) ...........5-8

Table 5-2. Z-Test: Two Sample for Means................................................................... 5-10

Table A-1. Organizational Representation: Ontologically coded socio-technical networks

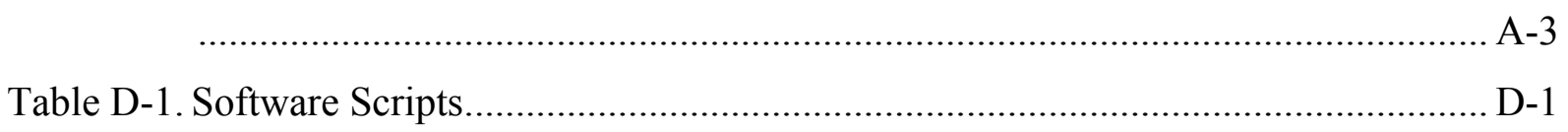




\section{LIST OF FIGURES}

Figure 1-1. Motivation for this Research ..................................................................... 1-5

Figure 1-2. Architecture of Simulation and Experiment Model ................................... 1-38

Figure 2-1. Comparison of average values for Organization Complexity variable over

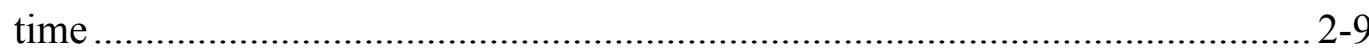

Figure 2-2. Comparison of average values for Team Complexity Alpha variable over time.

Figure 2-3. Comparison of average values for Team Complexity Beta variable over time

Figure 2-4. Comparison of average values for Infrastructure Support Alpha variable over time.

Figure 2-5. Comparison of average values for Infrastructure Support Beta variable over time

Figure 3-1. Construct Relationship Model (Standardized coefficients as a percentage) ....3-26

Figure 4-1. OLS-based Construct Relationship Model

Figure 5-1. Pearson correlation of two independent variables: Combined dataset..... $5-8$ 


\section{CHAPTER 1: INTRODUCTION}

Post-merger integration may be the most challenging organizational process to study. Understanding this intensely sensitive process is critical to organizational success, yet it remains under-studied by scholars; its inherent complexity can be overwhelming. This study embraces this challenge by systematically unraveling the complexities of post-merger integration. It signifies a start to a much deeper understanding of post-merger integration dynamics, at a level of fidelity not previously available.

This chapter presents the motivation for the study by explaining its rationale from a broad range of standpoints. To follow are several diverse sub-sections involving: a condensed overview of the entire chapter, a rationalization of the research and the problem statement, a survey of the relevant phenomenological support, a presentation of essential methodological matters, a synopsis of the three constituent studies, and a portrayal of the expected outcomes arising from the study.

\subsection{Overview}

This Overview Section provides a condensed form of Chapter 1. It presents only the essential facets of the study which are necessary to understand the central theme and some of the critical subtleties of this research. Foremost is the research problem statement.

\subsubsection{Problem Statement}

Organizations are under intense pressure to continually grow (Penrose, 1959). According to the resource-based view of the organization (Wernerfelt, 1984), organizational growth entails adding to organization's pool of existing resources, tangible or intangible. These resources can be added either gradually through organic development, or rapidly through inorganic extension. The inorganic approach typically involves a merger with, or an acquisition of, another organization (Capron \& Mitchell, 2012) that holds the sought-after resources. A merger-or acquisition-has high-degree of execution-risk inherent in its boundless complexity. Successful mergers are infrequent (Harrison, 2007); failure rates are 
markedly high. Optimists claim as many as 50\% are unsuccessful (Porter, 1987), while others assert as many as $80 \%$ are failures (Marks \& Mirvis, 2001). A poor post-merger integration process is often to blame (Marks \& Mirvis, 2001). Moreover, it is a problematic human integration - commonly labeled as a "culture conflict" - that is frequently the underlying reason for a troubled integration (Marks \& Mirvis, 2001).

Planning and managing the post-merger integration process poses an immense challenge (Harrison, 2007) to managers. Organizational stakeholders anxiously anticipate the post-merger outcome as the underlying organizational processes of the period are crucial to meeting the strategic goals of the merger, but these processes are extraordinarily complex and unstable - perhaps, the outcomes may even be somewhat random. Managers are expected to respond confidently and precisely to stakeholder questions, answers of which are comprised of highly complex forecasts of the organizational future. Will the two cultures endlessly clash or will they effortlessly coalesce into one? Will the combined organization actually perform at the expected level, that is, when will it reach its synergetic potential? Presently, there is inadequate understanding and scientific theory of post-merger integration dynamics to make science-based forecasts about such future outcomes.

To respond to the calls for forecasts, managers are left to rely only on their personal experience from previous mergers, and on often-minimally-informed, situational guesswork. In most situations, managers are cognizant only of a few, basic and observable, structural and cultural characteristics of the two organizations; in addition perhaps, they may know some of perceptible organizational history. Given the availability of only limited information, to forecast what actually will transpire after the two organizations merge is left to just crude speculation. Recognizing the limits of this ill-fated setting, this research endeavors to offer a scientifically-based response to a basic and highly-practical question, formalized as:

To what extent do antecedent, structural and cultural characteristics of two merging organizations relate to the post-merger outcomes of the combined organization? 
While the answer to this particular question may prove to be highly circumstantial, the practical problem that this study highlights, and scientifically addresses, is our present inability to provide a scholarly- and scientific-based response to a reasonable stakeholder question. This study endeavors to enable managers to provide such responses. Figure 1-1 graphically illustrates an overview of the basic structure and motivation underlying this research.

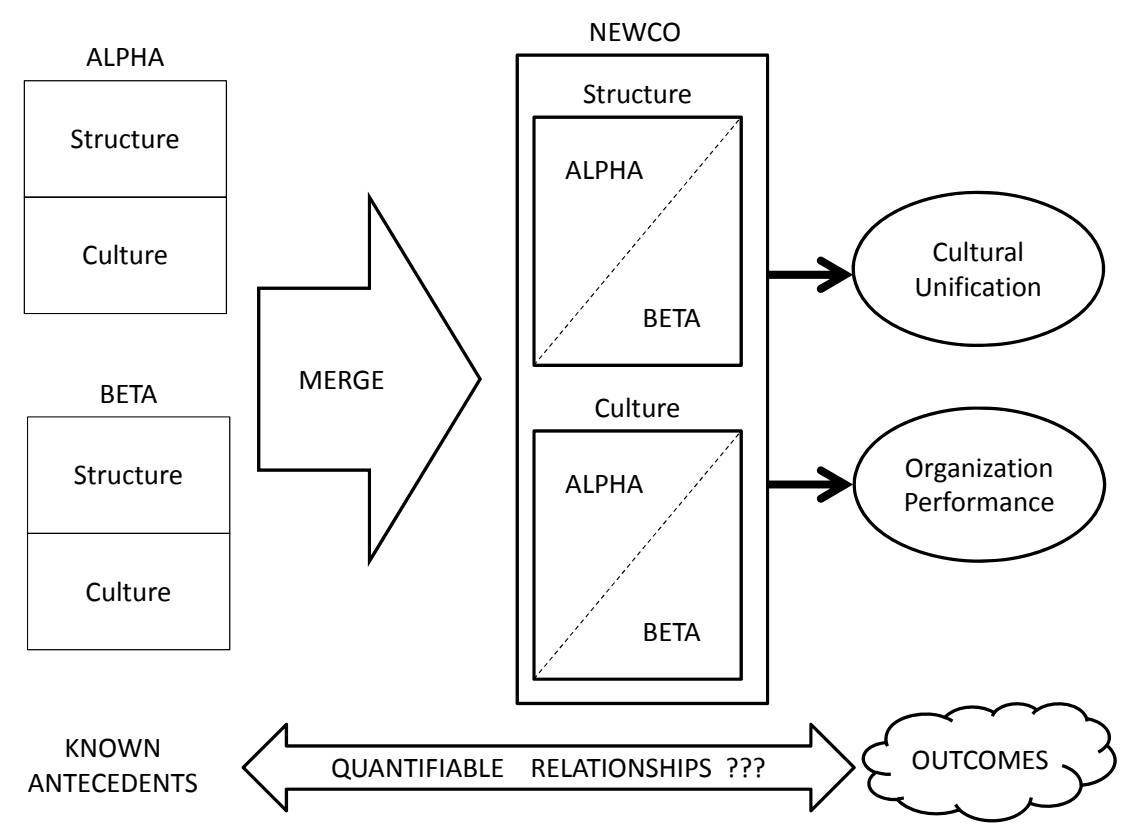

Figure 1-1. Motivation for this Research

\subsubsection{Phenomenological Exploration}

Both sets of phenomena, the post-merger integration outcomes and the antecedent characteristics of the pre-merger organizations, are complex concepts that require scholarly exploration of their own. With regard to post-merger outcomes, this study concentrates on two prominent concerns: (a) the degree of cultural unification, and, (b) the organization's performance. In regard to the antecedent features, in focus here are characteristics that can be classified into two types: (a) structural, and (b) cultural. In an empirical study, scholars identified the task integration and human integration as being two distinct processes of the post-merger process (Birkinshaw, Bresman, \& Hakanson, 2000); this study supports such a 
finding. Herein, Structural consists of straightforward, managerially direct-controlled features such as: (a) organization- and team-level complexity, (b) the structure of work teams, (c) taskspecialization of the workforce, and (d) the level of infrastructure support provided by the organization. Cultural includes more complex characteristics that are less directly-controlled by management and are revealed in individuals' behaviors, such as: (a) individuals' predisposition for seeking information, (b) sharing information, and (c) the individuals' focus on the assigned task.

These phenomena - the post-merger outcomes and the pre-existing characteristicsare integral facets of an organization's learning system. The characteristics of the organization affect, to some unknown degree, the dynamics of organization's learning process. Moreover, the learning system ultimately affects the performance of the organization. Thinking more abstractly, the learning system is supported by the organization's information exchange network, which also structurally supports, to an extent unknown, the transmission of culture throughout the organization.

A fundamental driver of the two post-merger outcomes is the dynamics of information - or knowledge - diffusion within the combined organization; this process is therefore a critical phenomenological underpinning of this research. A collective and unified culture is conjectured to manifest itself in a group of people having the same set of knowledge, while performance can be inferred to be manifest as the degree to which one holds the information — or knowledge — necessary to perform an assigned task.

Culture is learned shared knowledge in a group (Reyes-Garcia et al., 2003) and is preserved through a socialization process (Schein, 1990) that involves transferring cultural information, through behavioral cues and artifacts, etc. The anthropological and cognitive views of culture (Duranti, 1997), regard culture as the sum total of a group's knowledge (Linton, 1940), consisting of the facts of the cultural norms, traditions, expectations, customary behaviors, etc. 
Learning can also involve knowledge that is relevant to the performance of a specific task. In this study, such task relevant concepts are referred to as task-knowledge. Like many other aspects of this learning model, exactly what constitutes a relevant concept of knowledge is highly abstract, although concretely it is specific information that is necessary to perform the given task, expertly. Herein, the performance level of a task is measured by the extent to which the performer holds the knowledge necessary to perform that task.

In previous research, knowledge transfer in mergers has been recognized for creating additional synergetic value in the combined organization (Haspeslagh \& Jemison, 1991; Capron, 1996); it has also has been pointed out that knowledge transfer can be the sole purpose of other organizational combinations such as joint ventures (Kogut, 1988). For example, in the Robbers Cave experiment (Sherif, Harvey, White, Hood, \& Sherif, 1961), the manufactured merger of two groups - teenage students on a camp outing — was found to have resolved into the creation of an entirely new and distinct culture arising from the previously two detached and dissimilar cultures.

\subsubsection{Methodological Matters}

Scholars have evaded taking on comprehensive research directed on the grand question addressed by this study, possibly, because the granularity of data necessary to forge a precise response is impractical to collect in a laboratory, or in the real-world. Moreover, mergers are a sensitive time in any organization and just having access to an organization during this time is a privilege available to only a very lucky few scholars. However, by employing contemporary research methods, this study overcomes such obstacles. The contemporary methods designed into this study provide a rich and extensive amount of data that serve as a viable proxy for the real-world, which allows this study to overcome the barriers presented to traditional researchers.

Given the extreme circumstances of an organizational merger, obtaining abundant amounts of useful and detailed data is impracticable using a case methodology; therefor in this research, computer simulation is employed to generate representative data as a substitute. 
The theory-grounded, computational organization model employed in this computer simulation is based on the view that an organization is an information system sustained by a social network of organizational actors. This combined model is a proxy for real-world organizations and supports the notion of information being the critical feature of an organization.

Using CONSTRUCT (Carley, Martin, \& Hirshman, 2009; Carley, 1990; Carley 1991; See Appendix C) computer simulation software, controlled experiments are carried out that allow for organizing the antecedent characteristics as control variables, and the two outcomes as dependent variables. After running multiple replications of each factorial-designed experiment, the data can be transformed into mathematical models via linear regression techniques. These regression models implicate the strength of each control variable vis-à-vis the dependent variables, namely the cultural integration and organization performance.

\subsubsection{Constituent Studies}

This study is presented as a unified body of work, though it is comprised of three constituent studies that are each similarly designed, but are reported separately as independent research articles; each focuses on specific aspects of the more comprehensive, A Behavioral Theory of the Merger, provided in this overarching study. The three studies have each have different tactical research questions, though each has an identical methodology and experiment design; fundamentally, only the investigational control variables differ across the respective experiments.

The first study (Study One; presented as Chapter 2) titled, "Post-Merger Knowledge Transfer: Examining Antecedent Structural Complexity", examines the performance of the merged organization as it involves the pre-established structural-specific characteristics such as organizational complexity, team complexity and infrastructure support. It explores the consequence of the organizational size and the size of work teams, as well as the availability of technology to assist the information flow throughout the human-based information 
network. The findings of this study establish the strength of these complexity-related antecedents influence on the organizational performance outcome.

The second study (Study Two; presented as Chapter 3) titled, "Post-Merger Integration: Effects of Task Structure and Motivation to Exchange Information on Organization Performance", examines the performance of the merged organization as it involves both structural and cultural characteristics, such as the degree to which employees specialize, the level of team interdependence, employees' willingness to seek information, and the employees' willingness to share information. The findings of this study establish the strength of these cultural antecedents on the organizational performance and cultural unification outcomes.

The third study (Study Three; presented as Chapter 4) titled, "The Effects of Legacy Organization Culture on Post-Merger Integration”, examines the performance of the merged organization and the cultural integration as it involves the pre-established structural-specific characteristics such as organization- and team-level cultural complexity, and motivational characteristics such as exchange-motivation outreach and task-orientation. The findings of this study establish the strength of these motivational antecedents on the organizational performance and cultural unification outcomes.

\subsubsection{Outcomes: Scholarly \& Practice}

This study formulates a comprehensive A Behavioral Theory of the Merger, which offers a wide-ranging, scientifically derived description of the quantifiable relationships between pre-existing structural and behavioral characteristics of the merger partners and the cultural and organizational outcomes, post-merger. This theory provides not only a rankordering of the focal characteristic according to the direction and level of the structural and motivational features, but it also offers a comprehensive set of mathematical models, which allow for further scholarly experimentation and analysis. Moreover, these mathematical models can provide direction, if not an entire framework, for empirical study of the postmerger integration process at a later time and by others in the academic community. 
This A Behavioral Theory of the Merger study goes well beyond its scholarly origin and theoretic priorities; it provides tangible benefit to post-merger-integration practitioners. Since the theory includes a mathematical model that is scientifically valid for prediction, practitioners can utilize the model in a real-world setting. By recognizing a mathematical correlation between this array of features and the outcome during a post-merger, managers can better assess a proposed merger and better structure a plan for the integration stage. Taking what is known and effectively mapping it to what is wanted to be known, is invaluable. To date, this linkage has not been adequately accomplished and most certainly, a quantifiable link is not available to scholars.

\subsubsection{Forward}

The following sections in this chapter continue to serve as an introduction to this study. The ensuing discussion delves deeper and more extensively into each of the chief components of the study, beginning with a discussion of the problem being addressed.

\subsection{Problem Statement}

This study aims to address a persistent problem affecting organizations during a time of extraordinary complexity and pressure: the chronic failure of post-merger integration. This problem is described, in detail, in this section, which also accentuates the distinctive complexities of achieving effective post-merger integration. Moreover, an admission of the present incapacity to adequately forecast post-merger integration outcomes will be provided. This section concludes with a declaration of the study's formal research question.

\subsubsection{Unending Pressure for Business Growth}

Organizations are under immense pressure to grow continually (Penrose, 1959). Largely this pressure originates from shareholders' need for financial returns from their investment to exceed the market return-rate; however, other influences are less clearly defined - but can be just as forceful—, such as managements' self-interests, industry competition, or external mandate (Capron \& Mitchell, 2012). Towards their performance 
goal, organizations seek to accomplish growth in an efficient and effective manner (Burton, DeSanctis, \& Obel, 2006); and, in accordance with the lightning speed of today's capital and investment markets, organizations need to show evidence of their growth - quarterly.

To support organizational growth-regardless of how it may be measured-requires adding resources to the organization's asset-base, be they tangible or intangible resources. Employing the resource-based view of the organization (Wernerfelt, 1984), the bundle of assets under its control greatly affects the organization's competitive advantage. Such resources include capital investments such as machinery and buildings; and, intangibles include computer software, patents, etc. Of course, human resources - the people as well as their knowledge and skills - are also included in this bundle of resources (Lin, Hung, \& Li, 2006). In the case of human resource accumulation, management focus on associated risks is essential (Bryson, 2003) and is critical to procurement success. One influential measure of success is the ability of management to positively distribute and integrate organizational knowledge among the employees. Attention to risks associated when amassing hard assets and resources is more deterministic and thus poses much less business risk than acquiring soft resources, such as innovative thinking, which have a greater risk profile.

\subsubsection{Organically Build, or Inorganically Buy}

Additional resources can be amassed either gradually through organic development, or swiftly through inorganic expansion (Capron \& Mitchell, 2012). Organic growth is a slower option than inorganic as a merger or acquisition can quickly resolve the resource requirement. After analysis of the three generic capacity-growth options: building, borrowing, or buying (Capron \& Mitchell, 2012); organizations occasionally decide to buy capacity in the form a merger or acquisition as it is an immediate satisfier and often can even be a least expensive option.

Implementing the inorganic approach usually involves an organizational merger or acquisition. The risk-reward profile for an accelerated strategy such as a merger is highly 
leveraged. The downside risks are far-reaching and can affect all types of stakeholders in momentous ways.

\subsubsection{Merger Process}

In the two-stage view, the phases of a merger are the deal process, and the post-merger integration process; each has a vastly dissimilar operational-risk profile. When insurmountable problems arise, the deal stage has the inherent advantage of being retractable, whereas the integration stage is not - after the deal, the new organization is fully committed to the integration process. If during negotiations, a deal sours it can be abandoned, though there can be significant financial and reputational loss; which nevertheless, typically can be amortized and likely dissipates over time. However, when problems arise in the integration stage there is no going back; the post-merger integration must succeed or the merger fails.

The two-stage process of carrying out a merger has high-degree of execution-risk inherent in the complexity and uncertainty of the second stage, the post-merger integration process. Post-merger integration comprises the melding of the various systems: financial, technology, human, work, value-chain, socio-technical, etc. These systems can be quite deterministic and thus have a high degree of certainly associated with them; however, the human system is exceedingly stochastic and has an immense level of uncertainly associated with them.

\subsubsection{Chronic Failure of Mergers}

Successful mergers are infrequent (Harrison, 2007): Success rates are low; Optimists claim as many as 50\% fail (Porter, 1987), while others assert as many as $80 \%$ are judged as a failure (Marks \& Mirvis, 2001). Failure can be costly, both in financial and human terms. Mergers fail for the same reasons that any organization fails to accomplish its objectives. There are a multitude of factors that contribute to failure, or in contrast, to merger success.

Casually referring to academic literature and news accounts, there are as many reasons for failure as there are case studies. Empirically, assuming the financial terms of the merger 
were sound, most often a conflict between the two organizational cultures is blamed. Indeed, by definition, every culture differs in their respective, abundant characteristics, both minor and major. In the business-as-usual case of an everyday organization, cultural conflicts within the subgroups, e.g., between the sales department and the marketing department, indeed cultural failure can be anticipated in most cases, merger or not. While spreadsheets can calculate the rather deterministic financial outcomes, the human dimension of a merger is much more complex, stochastic and, thus, less foreseeable.

Designing or overseeing the post-merger integration process can be an immense challenge for management (Galpin \& Robinson, 1997; Harrison, 2007): unsuccessful integration is frequently the reason cited for a failed merger (Marks \& Mirvis, 2001). In the distant past, little was yet known about the factors affecting the integration merging organizations (Shrivastava, 1986). More recently, numerous factors have been acknowledged by scholars as - to some unspecified degree-leading to failure, or success, of the postmerger integration process. Aside from the economic, merger-type, and financial issues affecting the merger outcome (Wilamson, 1971), Bert, MacDonald, and Herd (2003) suggest that urgency and execution are imperatives to successful post-merger integration. Shrivastava (1986) views post-merger integration as having three interrelated tasks: coordination, control, and conflict resolution that must operate in harmony for success. Galpin (2010) offers that the deliberate, or accidental, process that management follows during the post-merger integration period is primary to the determination of integration success or failure. Mottola, Bachman, and Gaertner (1997) found that the integration patterns highly affected the commitment of the respective groups to the merged organization, and thus affects the outcome. Olie (1994) focused on integration in the international context, and found that the level of compatibility of the cultures is paramount and that the degree to which the two groups wish to maintain their organizational integrity is a primary factor of a successful cultural integration. In Weber and Camerer's (2003) laboratory experiment, it was confirmed that cultural conflict leads to merger failure. Epstein (2004) suggested several drivers of merger success that include socially-related factors such as a coherent integration strategy, a strong integration team, communication, speed of implementation, and aligned measurement. Moreover, Vaara (2002) 
reported numerous narratives that actors employ to recount their merger experience, including: rational, cultural, role-bound, and individualistic; this is indicative of the complexity and lack of clarity around just what factors lead to integration success or failure. In the research community, the question of identifying the drivers to success seems still comprehensively unresolved.

\subsubsection{Forecasting Post-Merger Outcomes: A Quagmire}

Planning and managing the post-merger integration process is an immense management challenge (Harrison, 2007). Stakeholders anxiously anticipate the post-merger outcome as the underlying organizational processes are essential to meeting merger goals, but are also extraordinarily complex and unstable - the outcomes may even be suspected as being random. Managers agonize over responding to stakeholders' basic and straightforward, yet critical and complex questions. Will the two cultures persistently clash or will they effortlessly coalesce into one? Will the combined organization actually perform at the expected level, that is, when will it reach its synergetic potential? Today, there is insufficient understanding and available scientific theory of post-merger integration dynamics to make reliable and sciencebased forecasts about such future outcomes.

Managers are left to rely only on their personal experience from previous mergers, and on often-minimally-informed, situational guesswork. In most situations, managers are cognizant only of a few, basic and observable, structural and cultural characteristics of the two organizations; in addition perhaps, they may know some of perceptible organizational history. Given the availability of only limited information, to forecast what actually will transpire after the two organizations merge is left to just crude speculation.

\subsubsection{Post-Merger Dynamics: Little Understood}

Presently, there is scarce understanding and scientific theory of post-merger integration dynamics for to forecaster to reasonably make a rational prediction about outcomes. Little is precisely known about to root causes of why merger integration fail, even 
in hindsight. Empirically, the mismatch of organizational cultures is blamed. The standpoint of this research is that beneath the vagueness of such statements, the fundamental foundation for failure may be that information is not flowing through the organization as would be the case in a healthy and productive organization.

Typically a merger requires the integration of human assets. Naturally, these assets are supplemented by precious skills and knowledge at the individual, group and organizational level (Lin, 2006). Ordinarily, management focus on these human resource assets and any intrinsic liabilities is vital to the outcome (Bryson, 2003), both near- and long-term. One approach to manage this task is to concentrate on the information diffusion throughout the new organization. Research indicates that knowledge and information exchange processes and dynamics during the post-merger integration period differ from normal times (Bresman, Birkinshaw, \& Nobel, 1999). It may be this information exchange aspect of the human resources that may be the underlying cause of integration and M\&A failure. Assuming the two organizations are entirely effective at information flow prior to the merger, which is a low probability assumption, the merger brings to the fore the communication conflicts that may arise between an in-group and outsiders in an out-group.

Organizations are comprised of multiple sub-systems that will experience an abrupt shock when two whole-level systems unify. The constituent sub-systems are thus perturbed and must endeavor to seek out a new equilibrium, optimal or otherwise. Complexity theory specifies that the initial starting point of any system greatly influences the future trajectory of that system. Mergers do present a shock to the organizational human-sub-system in numerous ways. There is an abrupt in-group out-group condition as well as sudden aura socio-technical uncertainty.

In the multifaceted case of an organizational merger, the initial starting points of the combined organization and its constituent sub-systems have buried within them a legacy and history that cannot be immediately forgotten; organizational memory lingers. 


\subsubsection{Overarching Research Question}

This research is motivated by the by recognition of the immense consequence of these human resources and the significance of the informational aspect of their day-to-day activities. Consequently, the theoretic and practical, information-based view of the organization is embraced herein. This implies that to evaluate the effectiveness of an organization one assesses the dynamics of the information flow in the organization. A goalseeking organization is considered effective to the extent that the information necessary for performing a task is held by those persons performing that task. Subsequently, a merger is deemed successful if the newly formed organization is able to get information necessary to accomplish tasks to those that are performing them. This research is future grounded on the notion that specific information necessary to perform some tasks deviates from legacy requirements during organizational disruption, particularly if the entire organization changes as a result of a merger.

\subsubsection{Post-Merger: Uncertainty}

To provide a responsive forecast to inquisitive stakeholders, managers are left to rely only on their personal experience from previous mergers, and on often-minimally-informed, situational guesswork. In most scenarios, managers are cognizant only of a few, basic and observable, structural and motivational characteristics of the two organizations; in addition

perhaps, they may know some of perceptible organizational history. Given the availability of only strictly limited information, to forecast what actually will transpire after the two organizations merge is left to just crude speculation.

Integration managers typically focus on two primary indicators of integration success: Organization performance and cultural integration. Organization performance is paramount both in the immediate and longer-terms, while reaching congruence with respect to cultural integration usually is allowed a longer time-horizon. Appropriately, this research specifically concentrates on these two indicators of post-merger integration progress and success. 
Researching post-merger integration presents systemic practical and logistical challenges to academics. Financial and economic aspects are rather straightforward to study as quantitative data are ready-made and easy to access, gather and analyze. On the other hand, human social aspects are extremely difficult to study. The human dimension is overly complex and constantly evolving below the surface. The structure of the human group changes, as do aspects of the individuals within. Moreover, the newly merged organization is entirely new, without a tractable history that can be used to estimate the future. The time in which a merger is in progress is a sensitive time for the organization and results-driven managers are reluctant to allow a researcher into their systems to investigate, so empirical and case-based research is difficult to carry out.

Recognizing the limits of this ill-fated setting, this research endeavors to offer a scientifically-based response to a basic and highly-practical question, formalized as:

To what extent do antecedent, structural and motivational characteristics of two merging organizations relate to the post-merger outcomes of the combined organization?

While the exact answer to this particular question may prove to be highly circumstantial, the practical problem that this study highlights, and scientifically addresses, is our present inability to provide a scholarly- and scientific-based response to it. This study endeavors to provide such a response.

\subsection{Phenomenological Exploration}

This research is fundamentally grounded in exploring the dynamics of organization learning during the special situation of post-merger integration. The manner by which the factions of the new-combined organization learn from one another once they formally merge is conjectured to be no different than under usual circumstances; the learning processes do not change during the integration process. However, the circumstances surrounding the learning during post-merger integration are vastly different. There is a more distinct organizational fault-line inherently present at the opening moments following the consummation of the 
merger. The two sides of the organization may have very different antecedent characteristics that to some varying degree may affect the learning occurring during the integration process. By following the cultural unification and organization performance outcomes, there is a window into these dynamics that can be used to study the inner workings of the organization.

This section provides an introductory, scholarly discussion on perspectives of organization learning, the two outcome metrics under examination, and the focal antecedent characteristics of the merging organizations.

\subsubsection{Organizational Learning}

An organization can be regarded as a repository for a body of knowledge (e.g., cultural tendencies, operational procedures, etc.) that is unique to any other organization. This body of knowledge is an important influence on the performance of the organization (Baum \& Ingram, 1998; Darr et al., 1995; Epple, Argote, \& Murphy, 1996) and can result in having a competitive advantage (Argote \& Ingram, 2000; DeLong \& Fahey, 2000). Moreover, an organization is a social construct designed as an instrument fashioned to handling information; abstractly, perhaps it is an information processing machine. Within its boundaries, an organization is fundamentally an information processing system, with highly complex internal dynamics.

The organizational processes supportive of information exchange, or knowledge transfer, are "a basis for competitive advantage" (Argote \& Ingram, 2000, p. 150; Zander \& Kogut, 1995). Consequently, information exchange should be a chief concern of management during post-merger integration, since mergers are usually consummated for the purpose of improving competitive advantage (Bruner, 2004). The exchange of information among the members of an organization is critical to individual and organizational performance from both social and actionable perspectives, and supports central concepts such as organizational learning (Argote, Gruenfeld, \& Naquin, 2000). In the usual sense, knowledge transfer refers to the transferal of existing "reservoirs of knowledge" (Argote \& Ingram, 2000, p. 153) across organizationally-bounded groups; however, this convention is changed to refer to such 
transfer occurring "within a newly-combined group" rather than across. Pre-merger groups will hold unique reservoirs of knowledge such that the subset of the constituent elementse.g., the tactic and extrinsic skills, contextual practices, and routines-, are particular and specific to their group; this holds nonetheless, even if two groups perform the same functional tasks; every organization has unique variances in its work processes, procedures, etc.

Considering the information processing facet of an organization, actors interact with one another as a matter of routine, i.e., they socialize. In the course of this repetitive socialization process, actors purposefully exchange information with one another, intentionally or unintentionally. They pass information either through direct person-to-person communications or through more indirect and contemporary forms, such as electronic emails and broadcast media (Dooley \& Corman, 2004). Keeping with our abstract understanding for information, the process by which information is exchanged with others, e.g., verbally, visually, even subconsciously, should also be held as being highly abstract.

Subscribing to the Carnegie School of Organizational Theory (Cyert \& March, 1963; March \& Simon, 1958; Simon, 1957), consider that the organizations information-processing capability is a critical underpinning for decision making and performance. There have been many attempts at precisely defining the term information without settlement on any concrete consensus (Case, 2007); herein, only abstractly modeling the real-world, the meaning of the term is preserved, as remaining highly-abstract. For example, Chaffee's (1991, p. 9) suggestion that information is "anything that exists psychologically for a person" would be a practical guidepost to idealizing the notion of information within the confines of this study.

Effectively combining the organization's human resources, including their embedded knowledge, is essential to establishing post-merger success in the immediate and longerterms. One approach to this task is to focus on the information diffusion throughout the new organization. Research specifies that knowledge and information exchange processes and dynamics, during the post-merger integration period, differ from that of normal times (Bresman, Birkinshaw, \& Nobel, 1999). It may be this information exchange aspect of the human resources that may be the underlying cause of integration and M\&A failure. 
The process of diffusing this knowledge throughout the various actors in the organization varies widely (Argote, 1999). In the case of an organizational merger, success often requires that the pre-existing knowledge held by both organizations must be diffused widely throughout the new organization (Heo \& Yoo, 2002; Peltoniemi, 2007) to capture and maximize post-merger performance.

In the special setting brought about during post-merger integration, effective knowledge transfer is paramount. In order to realize the newly expected post-merger performance level, the previous disjoint knowledge reservoirs must ultimately meld into a single pool of shared knowledge - a post-merger knowledge reservoir united within a single group. As part of the pre-merger planning, finances can easily be combined in an orderly and predictive matter; however, in the realm of an organization's knowledge-base, this integration of human knowledge is more of a complex and dynamic --perhaps chaotic-process (Szulanski, 2000), as opposed to the obviously-important but comparatively less-dimensional legal and accounting transactional events.

Recognizing culture as consisting of the sum total of a group's knowledge (Linton, 1940) - involving of the facts of the cultural norms, traditions, expectations, customary behaviors, etc- , the learning process itself can lead to a change in the culture itself. For example, learning may spread a minority-held custom among the larger group-as what occurs in popular social culture-, thus changing and redefining the accepted norms of the majority and even the entire group. In this study, the learning process does indeed change the culture of the entire group as the actors transmit their culture to dissimilar others, though what a group culture actually is can be a highly subjective matter.

\subsubsection{Post-Merger Integration Outcomes}

This research focuses on two outcomes of managing an organizational merger: (a) the integration of the cultures, and, (b) the performance of the organization. These two metrics are often indicative of merger success. The first outcome is an indication of cultural unification of a group, its cohesiveness as a work unit. Just as new member must assimilate into a group 
when being first introduced into the group, two merging organizations must integrate by either: (a) staying separate, (b) forming an entirely new culture, or (c) melding into one of the original cultures. Regardless, any social interaction among members of two groups will lead to exchange of culture and perhaps, over some length of time, some morphing of the cultures will result into an entirely new culture. The second outcome is organization performance which is a central metric for evaluating any organization; it is often the principal metric used to gauge the value, worth, or success of an organization. Its importance is clear and cannot be overestimated. For this study, the concept of integration of cultures is grounded in the notion culture-knowledge, and the performance of the organization is based on the notion of taskknowledge; these two ideas are explained below. Both are artifacts of the underlying organization learning occurring in the newly merged organization.

\section{Integration as Culture-Knowledge}

A collective and unified culture is conjectured to manifest itself in a group of people having the same set of knowledge, while performance can be inferred to be manifest as the degree to which one holds the information — or knowledge — necessary to perform an assigned task. A central feature of an organization is its unique culture, which can be the root cause of failure (Vaughn, 1996), "indigestion” (Schein, 1990, p. 117), or success. Furthermore, an organization-level culture can have within it differing sub-cultures at the work-group level (Van Maanen \& Barley, 1985), consisting of both similar and dissimilar characteristics. The culture of the merging organizations affect the post-merger integration outcome; for example, an organization's formal structure has been shown to affect these dynamics (Frantz \& Carley, 2010), although the full impact of the informal structure remains an open area for investigation (Frantz, 2012).

Culture is learned shared knowledge in a group (Reyes-Garcia et al., 2003) and is preserved and strengthened through a socialization process (Schein, 1990) that involves transferring cultural information-implicitly or explicitly—verbally or through behavioral cues and artifacts, etc. The anthropological and cognitive interpretations of culture (Duranti, 1997), regards culture as the sum total of a group's knowledge (Linton, 1940) consisting of 
the facts of the cultural norms, traditions, expectations, customary behaviors, etc. A contemporary example of such a norm in the organization setting is when and in what circumstances it is acceptable to use email instead of using direct verbal communication (ElShinnawy \& Markus, 1998; Rowe \& Struck, 1999), or even submit to using social media. This knowledge of one's culture is held by each member of a group or organization in the form of numerous, specific knowledge concepts (D’Andrade, 1995; Kroeber \& Kluckholm, 1952; Romney, 1999). The members embedded in a culture intimately know detailed aspects about their culture which are implicitly and explicitly shared and reinforced with other ingroup persons. Often such knowledge is held closely within the group and can sometimes intentionally be withheld from out-group persons in order to exclude unwanted others from the group. The perspective that a specific culture itself is a pool of shared and distinctive cultural information that is uniquely held by members of a specific group (D’Andrade, 1981), and that such information is not necessarily known or embraced by others outside of that particular group (Cremer, 1993). Note that in practice, it may not be in the strategic interest that the combined organization meld the two pre-existing cultures into one (Vestring, King, \& Rouse, 2003).

An important characteristic of a group culture is the affect culture has on group- and individual-level motivation. It has been established in cognitive research that individual behavior is influenced by one's environment and that it follows that group culture is one dimension of that environment; scholars have found that indeed culture can influence the motivation of an actor (D’Andrade \& Strauss, 1992; Munro, Schumaker, \& Carr, 1997). Specific to an organizational setting, Moon (2000) links the group members' commitment and

the culture of the group that leads to it. O'Reilly (1989) also makes the connection in a managerial oriented study that suggests social control in an organization can affect the culture to then promote greater work motivation within the group's members.

\section{Performance as Task-Knowledge}

To evaluate the progress of the organization during the post-merger integration period, thus to measure performance, there is a focus on task-knowledge capabilities within the 
merged organization. To measure the amount of knowledge that has been transferred not from a generic perspective, but from the qualitative perspective. That is to say, this is measuring the knowledge transfer for only those concepts that have value to the specific members of the organization, necessary to perform their work tasks, given the task-assignment structure of the individual work units.

In this study, attention is directed on knowledge that is relevant to the performance of a specific task, though other information can be vitally important, e.g. to advance group innovation (Inkpen \& Dinur, 1998). These task relevant concepts are referred to as taskknowledge. Like many other aspects of this model, exactly what constitutes a relevant concept of knowledge is highly abstract although it steadfastly is some specific information that is necessary to perform the given task expertly. Utilizing a simple example used in organization learning research (Argote \& Ingram, 2000): in a pizza-making task knowledge of how to toss the dough is relevant to making pizza, as is knowledge of how to combine the ingredients making the pizza sauce; while, knowing how to clean an over may be useful to a pizza maker, it is not necessarily essential to being a world-class pizza maker (cleaning the oven might be abstracted as being a separate task, perhaps).

Members of a group each bring different expertise, knowledge, and information into their group (Jackson, 1992) and the distribution of the knowledge within a group varies (Liang, 1994). The knowledge held by a group directly influences the performance of the group (Shaw 1981; Steiner 1972; Stasser \& Stewart, 1992). The distribution of the knowledge within the group can be deliberately managed (King, 1999) and greatly affects group performance (Rulke \& Galaskiewicz, 2000). How broadly the information is distributed and the amount of information shared within the group has been shown to affect the group performance (Stasser, Taylor, \& Hanna, 1989; Stasser \& Titus, 1985, 1987). Moreover, there can be complicated social dynamics occurring within a small group when it comes to exchanging such information (Deeker, Stokman, \& Franses, 2000). Localized knowledge common to a specific group can face natural difficulties being transferred outside of that group (Almeida \& Kogut, 1999), unless there is exchange of personnel among the group, such 
as within an industry, for example; in our case herein, melding human assets within a newly combined organization.

Naylor and Dickson (1969) offer that a factor of team performance is the structure of the task itself and that the complexity of the task is then based on the amount of information required to perform that task. This similar perspective that task-performance is directly associated with task-knowledge, which is also aligned with other research (Beirly \& Chakrabarti, 1996; DeCarolis \& Dees, 1999). It follows, all other aspects being equal that the more task-specific knowledge an actor has for performing a given task, the better the actor will be at performing that task, and greater the amount of knowledge necessary for performing a given task, the higher the degree of difficulty will be for performing the task. Tasks can differ in the level of their complexity (Wood, 1986) and the number of knowledge concepts to a specific task according to the complexity of that task, thus suggesting that a more complex task takes longer to master. An illustrative example of this would be a neighborhood pizza shop: if a pizza maker has no knowledge of how to make pizza, it would clearly result in a disappointing meal, while a pizza-maker with expert knowledge of how to make pizza, you can reasonably be assured you will have a delicious pizza nearly every time. This task knowledge often can be transferred from person to person and from group to group (Darr et al., 1995).

This study focuses on the knowledge transfer in the combined, post-merger organization. In particular, the focus is on the task-specific knowledge that is unique to previously detached workgroups and that has become essential to newly formed groups, such that the knowledge is essential for workers to complete a given task. In some work teams, knowing how to access information contained in the group is essential (Stasser, Vaughan, \& Stewart, 2000). Research indicates that organization change can affect knowledge transfer, and thus performance (Lin, 2000). Since each organization has specific knowledge unique to itself, for the post-merger organization to fully integrate, this knowledge must be diffused to others in the combined entity. 
In some strategic mergers, the cultural integration of the organization may not be desirable. Often times, mergers are a way to add unique capabilities to the acquiring organization that are not present within, nor easily reproduced. These cultural capabilities can be inseparable from the target organization and could be at risk if pressures to adapt to the new host suggest altering the culture, even slightly; in this situation, the culture of the organization is the sought-after asset. Indeed, any change to that distinctive culture is undesirable for business performance reasons. Thus, the usual aim of post-merger integration being towards some level of cultural unification can be detrimental to the performance of the acquired and to larger goals of the combined organization.

A 2014 deal by the Chinese firm Geely acquiring the Swedish Volvo automobile company is an example of employing this non-integration strategy. The combined GeelyVolvo entity does not desire the integration of the two cultures. Essentially, the Volvo brand and the culture that manufactures and maintains the brand (and automobiles) are precious. The new Geely-Volvo does not plan to combine the distinctive culture of their acquisition with that of themselves, or vice versa. This example is representative of the common case where a brand-oriented merger may warrant a reversal from the typical situation where combining the cultures is desired, thus post-merger integration takes on more of a protective stance. A case where cultural integration destroyed the prized brand is the acquisition of the Snapple drink brand by Pepsi Co. in the 1980s. The Snapple drink quickly lost nearly all of its market value after the merger because the band was managed by a combined and integrated unit consisting of managers from both companies; however, this integrated team quickly destroyed the brand as the culture that brought the initial success to Snapple was integrated and thus changed to be ineffective. Had Pepsi Co., left the original Snapple organization intact and undiluted, it is likely the Snapple brand would have continued to thrive instead of wander for decades.

\subsubsection{Antecedent Characteristics}

In regard to the antecedent characteristics of the merging organizations, in focus here are numerous effectual characteristics, which are aptly classified into two types: (a) structural, 
and (b) motivational. These are predisposed factors, fixed characteristics at the time of the merger. The first type is structure, which is how an organization is organized and characteristically defines how it is unique (Mintzberg, 1979). The second phenomenon, motivation is a mediator between structure and performance. Both reflect social patterns that are known to affect information exchange within an organization (see Bresnen, 2003): (a) structure (Cowan \& Jonard, 2004; Reagans \& McEvily, 2003; Wickesberg, 1968), and (b) motivation (Szulanski, 1996). An organization's social structure situates actors who are within close social distance of one another into a position to easily exchange their knowledge with the close-by others. The specific constructs controlled in the virtual experiments will fall under one of these two categories; these are presented later in this Chapter, and in detail in the constituent articles.

\subsubsection{Structural Antecedents}

Structure is a distinguishing factor in determining an organization's performance (Burton \& Obel, 1995). As an organizational antecedent, Structural consists of straightforward, managerially direct-controlled features such as: (a) organization- and teamlevel complexity, (b) the structure of work teams, (c) task-specialization of the workforce, and (d) the level of infrastructure support provided by the organization. Information flows from actor-to-actor according to the social network in which the actors co-exist, e.g., Phillips, Mannix, Nealec, and Gruenfeld (2004).

Studies have shown that the structure of this network affects the dynamics of the flow of information throughout that social network. The social network is divided by paying attention only to the task-assignment network that the actor is a member of. The structure of this task-assignment network is a factor in the information flow and subsequence performance of the organization. The task-assignment structure in an organization is therefore an important feature of performance. For example, forming a large distributed team presents a moral hazard in the case of slacking or freeloading. The work breakdown and assignment structure is formalized as the task assignment structure for the organization. The task assignment structure is a function of the particular task that the business-line of the organization requires to be 
performed. There are two perspectives in structure (a) the actor perspective (actor specialization), and (b) that task perspective (team interdependence). The type of workers in important, e.g., independent knowledge workers (Kimberly \& Evanisko, 1981) as is the structure (Hinds \& Kielser, 1995).

\subsubsection{Motivational Antecedents}

Motivational includes more complex characteristics that are less directly-controlled by management and are revealed in individuals' behaviors, such as: (a) individuals' predisposition for seeking and sharing information, and (b) individual's task focus.

Information flows are affected by the motivations of the individuals involved in the potential exchange. The extent to which an individual is motivated, or willing, to seek information from another person, clearly affects the flow of information to that individual. Moreover, the extent to which an individual is willing to share information with another when requested also clearly affects the exchange of information between the dyad, and therefore in the aggregate, throughout the organization. However, it is unclear the extent to which the organization-level is impacted given the heterogeneity of the members within the organization. There is a dilemma people face when considering their information exchange strategy and have several strategies that can be undertaken (see Larsson, Bengtsson, Henriksson \& Sparks, 1998), that directly affects the informational and brokerage power of the individual and those they chose to share or not to share their information with. Motivation has several dimensions, but is important to knowledge transfer (Osterloh \& Frey, 2000) and to the success of the organization as a whole (Osterloh, Frost, \& Frey, 2002). "Selfish individuals want to maintain their monopolistic position as holders of idiosyncratic knowledge" (Osterloh, Frost, \& Frey, 2002, p. 66), although sharing with your competition may be a self-positive strategy (Hamel, Doz, \& Prahalad, 1989).

Knowledge sharing by individuals is critical to organizational success

(O’Dell\&Grayson, 1998; Osterloh \& Frey, 2000). Motivation is a factor in complete knowledge sharing both for the provider and recipient (Quigley, Tesluk, Locke, \& Bartol, 
2007) and the communication attitudes along with actors having task knowledge has been shown to affect performance (de Vries, van den Hooff, \& de Ridder, 2006). There is extant literature on social identity issue in a merger setting (Boen, Vanbeselaere, \& Cool, 2006; Clark, Gioia, Ketchen, Jr., \& Thomas, 2010; Hogg \& Terry, 2000; Ullrich, Wieseke, \& Van Dick, 2005; van Knippenberg, van Knippenberg, Monden, \& de Lima, 2002; van Leeuwen, van Knippenberg, \& Ellemers, 2003). Recently, the notion of a transitional identity (Clark, Gioia, Ketchen, Jr., \& Thomas, 2010) has been suggested: during the integration period, individuals may take-on a temporary social identity as they transition from their pre-existing social identity into establishing a new social identity in the combined organization. For the exchange to be effective there needs to be shared understanding and mental models (Ko, Kirsch, \& King, 2005). Moreover, knowledge sharing is positively associated with performance (Du, Ai, \& Ren, 2007).

\subsubsection{Constructs}

There are 14 phenomenological constructs involved in the experiments conducted within the three constituent studies. Each are explained in detail in the article(s) that they are used. For convenience, Table 1-1 provides a list of these constructs; for each, relevant scholarly references are provided, as well as an indication of the article(s) exploring it.

Organization complexity can be construed as the number of divisions, teams, or workgroups that subsume an organization. The greater the number of these subunits, the more coordination is likely to be necessary (and at several levels) and must therefore occur for the organization to perform its organization-level functions, i.e., complete the production of products and delivery of services. The greater number of these organizational subunits and thus the larger the number of possible cross-unit relationship, more complex an organization is; there are therefore many more coordination-related breakpoints that can cause failure, or opportunistically perhaps, create opportunities for greater efficiencies.

Team complexity can also be realized by the number of individual workers (employees, associates, members, etc) there are in one of these work units. The more 
members, the more human interaction and coordination occur within the group, which competes with the non-group members of communications bandwidth. By interacting with proportionally more members of the same work unit, the more likely it is that task-knowledge will be exchanged; however, given a greater number of work-group members, the probability that a specific task-knowledge concept that is of value to the receiver is transmitted is therefore reduced. This local effect is expected to overbear the dilution from non-same workgroup members because of the effect of a preference that an actor would communicate with another that is more similar to oneself.

Table 1-1. All-inclusive Listing of Constructs Involved

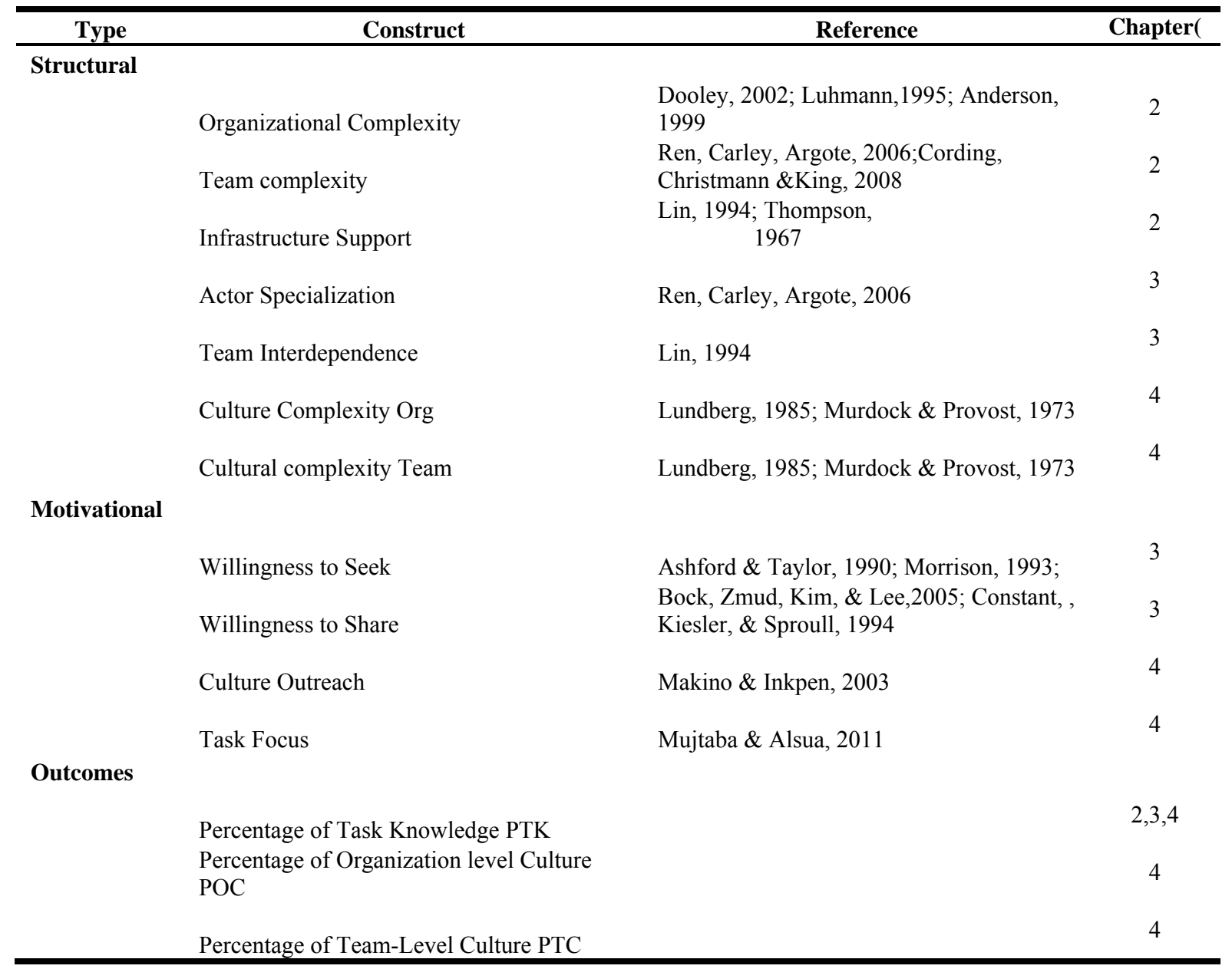

The level of infrastructure support in an organization is relevant for an investigation into task-knowledge transfer because having more technology in an organization can reduce 
the amount of task-knowledge that must be passed through person-to-person interaction for the completion of a given task. Instead, this necessary task-knowledge is stored in technological systems, such as employee manuals, web pages, posters on the walls, and computer-based training programs and databases. The exchange of existing task-knowledge from one organization to members of another, when embedded in technology, is void of many human issues that are not removed in a human-to-human knowledge exchange.

Actor Specialization is a value between 0 and 1 that indicates the fraction of the tasks that are assigned to an actor which are entirely unique to all other actors in the same team. That is, the Task node has only one tie to an Actor node in the Task-Agent matrix. Actor Specialization is the percentage of tasks assigned to the actor that are uniquely tied to them, relative to the entire number of tasks assigned to the same actor, thus a value of 0.0 implies that the actor is assigned exclusively to tasks that other actors in the same team are assigned, e.g., a track-and-field athlete who participates in the 400 meter and 1,000 meter relay races. Whereas, a 1.0 implies that the actor is assigned to solo tasks exclusively; they have no coworkers on their assigned tasks, e.g. a track-and-field athlete who runs the 100-meter and 400 meter sprint events.

Team Interdependence is a value that indicates the fraction of agents within a team that each previously unassigned task (after the actor specialization initialization process is completed) will be assigned to. A value of 1.0 indicates that each (non-actor-specialized task) task is assigned to the full set of all actors in the work-team, implying a highly collaborative task, i.e., all members of the team work together on a given task. A value of 0.5 indicates that $1 / 2$ of the work-team is assigned to work on a given task. Using the track-and-field example, this value would indicate the number of runners participating in a single relay race.

Cultural complexity can be regarded as the richness or exoticness of a culture and conceived as the number of attributes unique to a given culture; the greater the number of these unique attributes, the greater its complexity. This notion holds for culture complexity at both the organizational and the team levels. Every culture has some perceived level of complexity associated with it (Lundberg, 1985; Murdock \& Provost, 1973). Exchange- 
motivation is regarded as the members' enthusiasm for sharing information with others. Cultural exchange-motivation is the tendency for an individual to reach out to another to share information about their culture as well as their interest in accepting knowledge about a different culture from another. One's motivation to share and receive cultural knowledge is a characteristic of positive organizational citizenship (Jo \& Joo, 2011), which can be markedly influenced by the social structure of the organization (Lin, 1999; von Krogh, 2003). The tendency for sharing knowledge makes unique information accessible to others (Makino \& Inkpen, 2003); this information transfer enables cultural integration with and task performance to others in a group in which one identifies themself (Nahapiet \& Ghoshal, 1999). Task-focus orientation can be regarded as the tendency of an individual to focus their attention on, and therefore increasingly communicate about, tactical and formalized information over and above more casual non-task oriented information, such as when engaging in social gossip. In this study, the question is to what extent to which cultural complexity, at the organizational and team levels, cultural outreach, and task focus are factors in the organizational dynamics and performance during post-merger integration.

Organizational-level cultural complexity can be regarded as the richness or exoticness of a culture and conceived as the number of attributes unique to a given culture; the greater the number of these unique attributes, the greater its complexity. This notion holds for culture complexity at both the organizational and the team levels. Every culture has some perceived level of complexity associated with it (Lundberg, 1985; Murdock \& Provost, 1973). For example, the Japanese are branded as having many nuanced traditions in their behaviors, protocols, norms and social cues, while American culture might be considered as simpler and somewhat less nuanced and therefore less complex. Moreover, a metric conceived to quantify the complexity of a culture by counting the number of specific cultural traits that are specific to a given culture. Thus, the Japanese might have a value of 10, while Americans have a culture complexity value of 3 . Of course, this would be difficult and impractical in reality, so consistent with computational models that are working here within an abstract realm. Simply put, the greater the number of these underlying cultural traits, the more complex the culture is. If forced to craft specific values, an especially simple approach to expressing this metric could 
be taken: one could satisfy a metric value by using a weighed form of the Hofstede (1983) values.

Cultural complexity is of immediate relevance in the post-merger integration process. In order to unify two cultures into a single culture in NewCo, members of Alpha will be exposed to the specific cultural traits of Beta, and visa-versa-possibly spawning an entirely new culture, for NewCo itself. The relative difference (Hofstede, 1983) between the two premerger cultures, in particular the difference in the complexity, will lead to a more complex integration. This is to say that the greater the number of these concepts that must be diffused, the greater the amount of human interaction necessary for transferring these concepts from one individual to another. This may have implications for task performance because instead of an interaction exchanging task-knowledge, organizational culture concepts are transferred in its place. Therefore, the greater the number of these organizational culture concepts, the longer the time for complete culture-awareness to be reached at both the organization and team levels.

Team-level cultural complexity, similarly to organization-level culture complexity, can be regarded as a count of the specific characteristics that are unique to the culture of a specific work-team, relative to another such work-team in the organization; the greater the number of these concepts the more complex the culture is deemed to be (see the discussion above). Team-level cultural complexity has relevance in the post-merger integration process as within a specific NewCo team, the Alpha members will become exposed to the cultural concepts of Beta and vise-versa. The greater the number of these concepts that must be exchange and diffused throughout the team, the greater the number of dyadic interactions necessary for completing the total transfer of all these concepts from one individual to another. This may have implications for task performance because in a culture of high interaction exchanging task-knowledge, team culture concepts are transferred instead; thus, the greater the number of these team culture concepts, the longer the time for full dissemination to be reached.

Willingness to Seek is a fraction of the time-periods that an actor will be active in the interaction search process, i.e., the probability that a given actor will actively search for 
another actor to interact with in a given period. The variable takes on a value between 0.0 and 1.0 .

Willingness to share is the fraction of knowledge facts that an agent will consider to exchange with an interaction partner. This is a value between 0 and 1 , with the extreme value of 0 meaning that the agent will consider sharing $0 \%$ of the knowledge the agent has; to the extreme at 1.0, the actor will consider all of their known knowledge facts when interacting with a partner. From this set of knowledge, a uniformly random selection is made to determine the specific concept to be shared.

Exchange-motivation Outreach is regarded as the members' enthusiasm for sharing information with others. Cultural exchange-motivation is the tendency for an individual to reach out to another to share information they have with others. The tendency for sharing knowledge makes unique information accessible to others (Makino \& Inkpen, 2003); this information transfer enables cultural integration with and task performance to others in a group in which one identifies himself (Nahapiet \& Ghoshal, 1999). Some groups are quite proud of their culture and are self-energized to pass it on to others; they share who they are either directly, through stories, or through tangible artifacts such as posters on a wall. A high level of cultural outreach would suggest that individuals seek out others who differ from themselves and have exchanges that would have snippets such as "this is how we do it", or "we always do..." A low cultural outreach culture would result in overall less cultural transmission from the in-group to the unlike others, the out-group. For example, in an organizational culture that structures employee pay according to individual performance and is thus highly competitive, there could be less of this "free" advice being passed from one person to another, relative to a team-oriented culture supported by team bonus pay.

Task-focus orientation in a culture can be regarded as the tendency for individuals to focus their attention on, and therefore increasingly communicate about, tactical and formalized information. This would be opposed to a more relationship-oriented culture that communicates more casual non-task oriented information, such as when engaging in social gossip (Davis, 1973). One culture can be more task-oriented than another and then would be 
inclined to have more interactions involving task-knowledge exchange than other non-task knowledge. An example of a task-oriented culture might be an organization engaged in engineering design, or computer programming. A less task-focused culture might reside in an entrepreneurial organization. American culture is considered highly task-focused, while Japanese culture is considered less task-focused (Mujtaba \& Alsua, 2011). In academic research, it has been shown that a task-focus is more conducive to organization performance (Bass, 1967), though such findings are highly contextual.

Percentage of Task Knowledge (PTK) is the percentage is calculated by the number of task-knowledge concepts that have ties in the actor-knowledge network (the number of actual) divided by the number of unique actor-knowledge ties required (task-knowledge times actortask network times actor-knowledge).

\subsection{Methodological Matters}

This section presents a summary of method-related matters employed in the constituent studies. Experiment-level details are provided in the Methodology Sections each of the respective constituent articles, as per traditional research-reporting standards. Moreover, the Appendix Sections of this document provide extensive explanation and detail of the computational models and software employed in the three studies. When applicable in the discussion, the reader will be directed to these supplements.

\subsubsection{Data Scarceness}

Scholars have steadfastly evaded taking on comprehensive research concentrating on post-merger integration; feasibly, because the granularity of data necessary to forge a accurate response is essentially impossible to collect in the real-world. Moreover, mergers are a sensitive time in any organization and even just general access to the organization during this time is an opportunity to only a very few scholars. However, by employing contemporary research methods, this study overcomes such obstacles to deliver a comprehensive and scientifically-based response to this persistent, yet unexplored, and fundamental question. 


\subsubsection{Computer Simulation \& Computational Models}

In this research, computer simulation is employed to generate data as a proxy for laboratory or real-world data. Given the extreme circumstances of an organizational merger, obtaining abundant amounts of detailed data is unachievable using a case methodology; therefor in this research, computer simulation is employed to generate representative data as a substitute for analytic support. The computational organization model employed in the simulation is based on the view that an organization is an information processor supported by a social network of organizational actors. This combined model is a proxy for real-world organizations and supports the notion of information being the critical feature of an organization.

The computational model of an organization used for these experiments is grounded in the Construct theory of social behavior (Carley, 1990; Carley, 1991) as the theory is implemented in CONSTRUCT (Carley, Martin, \& Hirshman, 2009; Carley, 1990; Carley 1991). CONSTRUCT is agent-based simulation software developed and maintained by CASOS (Carley, Martin, \& Hirshman, 2009). CONSTRUCT is based on the Carnegie School of social theory: people interact with one another within a network of social-technical relationships and exchange information with others in the network. CONSTRUCT has been used extensively in academic and governmental research. For extensive detail about CONSTRUCT and the underlying computational model and theories, see Appendix I in this article; additionally, readers are referred to any of the numerous descriptions by the authors (Carley \& Frantz, 2009; Frantz \& Carley, 2009a, 2009b).

The representational model implemented within Construct is based on the metanetwork construct (Carley, 2002a, 2002b), which is a primary feature of Dynamic Network Analysis (Carley, 2003). Numerous networks are formed from three basic entity types: actors, knowledge and tasks. Broadly, the representation model is consistent with the PCANS model (Krackhardt \& Carley, 1998). From the three entity types, four types of networks are utilized. The first is the social network, which is an actor-to-actor relationship. The second is an assignment network consisting of actor-to-task relationships. The third is a task-to-knowledge 
network which represents the concepts that are necessary to perform each task. The fourth is the knowledge network which is an agent-to-task network representing what concepts each agent knows. Note: More in-depth details about this aspect of the virtual model is presented Appendix I.

The behavioral model embedded in the individual agents for this simulation also relies upon the CONSTRUCT model (Carley, 1991). The Construct model has underpinnings based on social theories such as Symbolic Interactionism (Blumer, 1969), Structural Interactionism (Stryker, 1980), and Structural Differentiation Theory (Blau, 1970). The agent behaviors in the CONSTRUCT model are grounded in the information-processing perspective of the Carnegie School (Simon, 1957; March \& Simon, 1958; Cyert \& March, 1963), which models people as being information seeking agents. Moreover, CONTRUCT incorporates socialinformation-processing theory (Rice \& Aydin, 1991; Salancik \& Pfeffer, 1978), which posits that what knowledge a person has is largely dependent on the information they have access to via their social network. In CONSTRUCT, agents purposefully engage in multifaceted discourse (Dooley, Corman, McPhee, \& Kuhn, 2003) and social interaction (Carley, 1986) to exchange knowledge with one another. Agents regularly seek out others who have knowledge that they do not yet hold, and consequently are also being sought out by those seeking their knowledge. The selection of an interaction partner is complex and independently occurring, thus the formation of social groups is a self-organizing phenomenon (Guerin \& Kunkle, 2004). This complex interaction behavior is played out continually within an organization and mimics real-world behavior at an abstract level, leading to an approximation of the information diffusion process within an organization. Note: More in-depth detail about this aspect of the virtual model is presented the appendix.

\subsubsection{Controlled Experiments}

These experiments are conducted within the realm of a computational laboratory (Burton, 2003); in short, all of the computer simulations are similarly designed but conducted independently. These simulations are based on a realistic organizational behavior and merger script. The scenario is much like a typical real-world case found in academic case-studies and 
is the script to which the computer simulation is guided and from which the virtual experiments are designed. Two independently conceived organizations, Alpha and Beta, are thrust into a merger to form NewCo. Alpha and Beta are envisioned to provide similar products and services that complete in the same market and thus it is a horizontal merger. Alpha (the acquirer) is seeking to gain a larger share of its market and hopes to gain economies of scale through various synergies by combining with Beta. Cost-cutting is not part of NewCo's forward strategy; moreover, workforce redundancies are not of concern and therefore layoffs not part of the virtual script.

At the start of a virtual experiment, when Alpha and Beta are actually merged, the components of each are commingled at the team-level task under the NewCo umbrella. Consequently NewCo consists entirely of the pre-merger characteristics and pre-existing social networks of Alpha and Beta. This situates NewCo as a possibly eclectic mixture of socio-technical sub-networks that have become intermingled and must now interact according to the proscribed social behavior theories contained in the computational model, i.e., Construct.

In this simulation two autonomous, goal-seeking organizations are combined into one under the pretense of an organizational merger. Again, the pre-merger organizations are branded as Alpha and Beta, and the post-merger organization as NewCo. Strategically, Alpha and Beta merge in order to increase market share and thus expect to gain from economies of scale and various other synergies. Cost-cutting is not part of the strategy and workforce layoffs are of no concern, so the removal of actors from the organization is not a part of this simulated case; this is to say that all of the employees from both organizations will become members of NewCo.

This fictional, yet typical, case is construed to be a horizontal merger, so structurally both organizations have corresponding functional sub-units, e.g., accounting, sales, H.R., I.T., $\mathrm{R} \& \mathrm{D}$, etc. Both organizations are established businesses and are stable in all conceivable aspects; notably, prior to the merger, each is operationally performing at its maximum level. Each has reached a state of being a coherent and effective collection of integrated work-team 
comprised of highly-skilled (relative to their assigned tasks) and high-performing actors, all working with group-level purpose towards maximizing their individual-, work-team- and organization-level performance. Also stable and maximized, is the intra-team social network (Frantz, 2012) among the actors with inter-team ties establishing a cohesive organizational unit; any actor in the organization can plausibly interact with any other.

NewCo -- thus previously Alpha and Beta as well -- is formally organized with a work-team structure that, through the efforts of individuals within the work-team unit, aims to accomplish an explicit set of tasks. Each actor, whom is assigned to a specific work-team, performs their assigned tasks to the extent in which they have the necessary knowledge to perform that specific task. Such knowledge is acquired from others who are (or may be) sharing their knowledge with other actors, via typical social methodologies such as conversation, emails, etc. While the NewCo actors all now are members of the same social and organizational setting, because of their organization of origin (Alpha or Beta), task assignments and unique work histories, there is a probabilistic expectation of a difference in knowledge being held by each individual.

At the initialization of each virtual experiment, Alpha and Beta have just recently been merged into NewCo. The actors of each are commingled into teams through their task assignments under the amalgamated NewCo structure. Consequently, NewCo initially consists entirely of the pre-merger characteristics and pre-existing social networks of the actors from Alpha and Beta, conjoined only at the point of task-assignment. This situates NewCo as an eclectic mixture of socio-technical sub-networks that have simply become fused. Individual actors, in this virtual environment, now function, within the new task assignment structure, according to the social behavior theories implemented in the ascribed computational model; this computational model is described below.

An organizational merger transaction is a single point-in-time event that involves combining the various socio-technical networks of Alpha and Beta to form NewCo. The respective organizational teams from Alpha and Beta are paired and combined at the start of the simulation run. Once NewCo is setup, as a facet of the simulation initialization process, 
the behavioral model described above is in full authority of the behaviors occurring within the simulation. This simulation does not differentiate any behaviors between running in an ordinary or a post-merger situation.

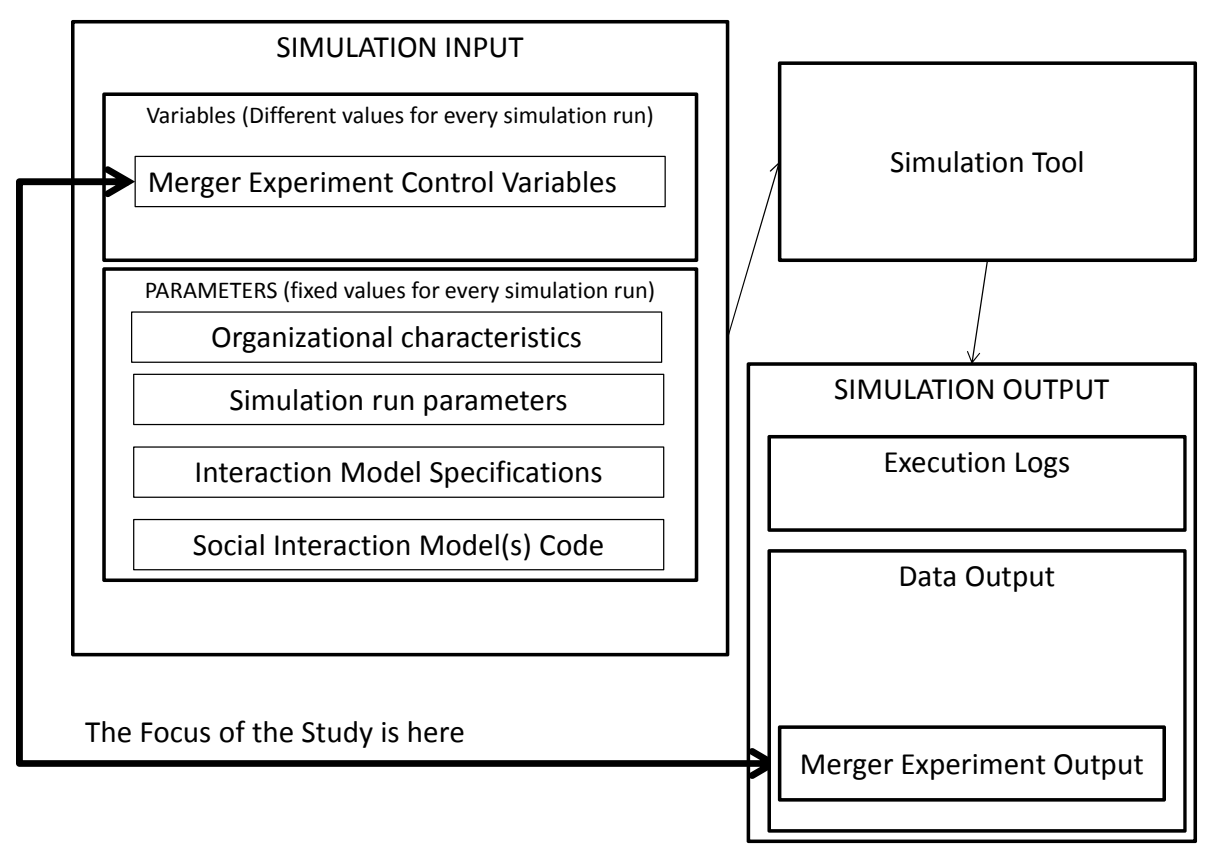

Figure 1-2. Architecture of Simulation and Experiment Model

\subsubsection{Construct Software}

The simulation software used is based on CONSTRUCT, which is a validated (Schreiber \& Carley, 2004a, 2004b) model of social behavior that has been used in numerous studies and is a powerful model for simulating organization behavior. Construct is grounded in the Carnegie School of social theory: people interact with one another within a complex network of social-technical relationships and exchange personally-held information, or knowledge, between themselves. CONSTRUCT, an agent-based model, also incorporates the notion of transactive memory in the actor-to-actor exchange. This is to say that people can transmit to their exchange partner, their knowledge of another person knowing of some particular fact or information. Note: More in-depth details about CONSTRUCT software and operational configurations are offered Appendix II. 


\subsection{Constituent Studies}

This research consists of three separate articles that, together, are the foundation of A Behavioral Theory of the Merger. Each study is a stand-alone scholarly work--though they have nearly identical methodology and experiment design--, yet they are intertwined to support the study's overarching research question. Each article centers on one of the research questions introduced below.

\subsubsection{Study One}

The first study entitled, "Post-Merger Knowledge Transfer: Examining Antecedent Structural Complexity" examines the performance of the merged organization as it involves the pre-established structural-specific characteristics such as organizational complexity, team complexity and infrastructure support. It explores the consequence of the size of the organization and the average size of work teams, as well as the availability of technology to assist the information flow throughout the human-based information network. The motivating research question for the study formally stated is:

What are the directional effects of the initial differences between two organizations, Alpha and Beta, on fulfilled task-knowledge transfer levels in the combined organization, NewCo, with respect to the relative degree of their (a) organizational complexity, (b) team complexity, and (c) infrastructure support?

Study One established five points: (1) Organizations with lower organization-level complexity will make greater, more noticeable progress in task knowledge diffusion early-on in the post-merger integration period. (2) Team-level complexity has a dynamic characteristically similar to that of organization-level complexity. (3) The relative difference in team-level complexity between the two original organizations has minimal effect on the task-knowledge transfer rate. (4) The level of infrastructure support positively affects the speed to which the organization reaches its task-knowledge saturation point. (5) The relative 
difference in infrastructure support between the two original organizations has minimal effect early on in the integration process, but becomes more prominent in later stages of the process.

\subsubsection{Study Two}

The second study entitled, "Post-Merger Integration: Effects of Task Structure and Motivation to Exchange Information on Organization Performance" examines the performance of the merged organization as it involves both structural and motivational characteristics, such as the degree to which employees specialize, the level of team interdependence, employees' willingness to seek information, and the employees' willingness to share information. The two motivating research questions for the study formally stated are:

Structure: What are the quantifiable effects of the initial differences between Alpha and Beta on Task-Knowledge Transfer in NewCo, with respect to the antecedent, relative task-assignment structure? This is meant to explore how the manner in which work is assigned to and distributed among workers affects the knowledge transfer outcomes.

Motivation: What are the quantifiable effects of the initial differences between Alpha and Beta on Task-Knowledge Transfer in NewCo, with respect to the antecedent, relative information-exchange motivation of organizational actors?

Study Two established three points: (1) managers may safely not be overly concerned about motivating the member of the acquired organization to share information with members of the acquiring organization, (2) an organization with lots of interdependent work-teams may require less attention during the post-merger integration than an organization made up of unconnected work groups, and (3) it may be just as effective to focus on information exchange only to a point of critical mass rather than attempting to attend to all members of the combined organization; the outcome may be just as positive. 


\subsubsection{Study Three}

Finally, the third study entitled, "The Effects of Legacy Organization Culture on PostMerger Integration" examines the performance of the merged organization and the cultural integration as it involves the pre-established structural-specific characteristics such as organization- and team-level cultural complexity, and motivational characteristics such as exchange-motivation outreach and task-orientation. The two motivating research questions for the study formally stated are:

Complexity: What is the effect of pre-established cultural complexity on the diffusion of culture and task knowledge in the combined organization?

Exchange Motivation: What is the effect of pre-established cultural exchange motivation on the diffusion of the culture and task knowledge in the combined organization?

Study Three established three points: (1) managers may safely not be overly concerned about motivating the member of the acquired organization to share information with members of the acquiring organization, (2) an organization with lots of interdependent work-teams may require less attention during the post-merger integration than an organization made up of unconnected work groups, and (3) it may be just as effective to focus on information exchange only to a point of critical mass rather than attempting to attend to all members of the combined organization; the outcome may be just as positive.

\subsection{Outcomes: Scholarly \& Practice}

While, post-merger integration research has previously been conducted in areas such as culture (Buono, Bowditch, \& Lewis, 1985), HR policies (Profusek \& Leavitt, 1984), structure (Mirvis, 1985), and internal social networks (Frantz, 2012), such a collection still leaves a scholarly gap. Missing is a comprehensive and predictive theory for studying, or 
rationally forecasting, the integration process. The purpose of this research is to provide such comprehensive theory in the form of A Behavioral Theory of the Merger.

The findings of this study have two chief patrons: scholars and practitioners. In a general sense, scholars seek to understand post-merger integration dynamics, and practitioners seek to forecast it. The results of this study benefit both.

\subsubsection{For Scholars: A Behavioral Theory of the Merger}

This study formulates a comprehensive A Behavioral Theory of the Merger, which offers a wide-ranging, scientifically derived description of the quantifiable relationships between pre-existing structural and cultural characteristics of the merger partners and the cultural and organizational outcomes, post-merger. This theory provides not only a rankordering of the antecedent characteristic according to the direction and level of the structural and cultural features, but it offers a comprehensive set of mathematical models, which allow for further scholarly experimentation and analysis. Moreover, these mathematical models can provide a route, if not an entire framework, for empirical study of the post-merger integration process at a later time and by others in the academic community.

A Behavioral Theory of the Merger consists of the findings from this study - some mentions previously in this Introduction chapter. For example, the conclusion that structure has more bearing on the post-merger outcome than does the cultural features is one that scholars to use to inform future research plans.

\subsubsection{For Practitioners: A Forecasting Tool}

The A Behavioral Theory of the Merger study, goes well beyond its scholarly origin and theoretic priorities, it provides tangible benefit to post-merger-integration practitioners. Since the theory includes a mathematical model that is scientifically valid for prediction, practitioners can utilize the model in a real-world setting. By recognizing a mathematical correlation between this array of features and the outcome during a post-merger this managers can better assess a proposed merger and plan for the integration stage. Taking what is known 
and mapping it to what is wanted to know, is invaluable. To-date this linkage is not known to have not been accomplished, and most certainly a quantifiable link is not available. An example of this would be to forecast that merging with one potential partner might take twice as much time to complete the integration than having merged with a different partner.

\subsection{Forward}

As presented in the earlier Introduction Section, this research endeavors to scientifically respond to the question:

To what extent do antecedent, structural and cultural characteristics of two merging organizations relate to the post-merger outcomes of the combined organization?

This question is noteworthy because while on the surface a merger is a swift and straightforward way to increase resources, most fail to accomplish their objectives. Mostly academics probe into mergers from an industrial, financial or legal perspective; meanwhile, the post-merger integration-particularly the cultural aspects - is often to blame for the failure. This study embraces the perspective that a merger is an exceedingly human endeavor, over and above being purely a financial and legal maneuver. In the financial and legal realms, a merger is rather deterministic, while the human perspective is chaotic, unpredictable, and stochastic. There is a plethora of financial, strategic and legal research on mergers while not enough research into the human aspects has been carried out; such research remains in its nascent stage.

The aim of this research is to develop scholarly theory and thus applicable managerial insight in the dynamics of the post-merger integration process. From this, practitioners can develop real-world integration strategies based on scientific and academic theoretic underpinnings, as opposed to just their empirical groundings. Moreover, practitioners can apply this research to pre-evaluate a real-world merger scenario for associated integration

risks, and perhaps opportunities; it also provides support for the adjustment of integration strategies during the actual integration process. 
This thesis investigates the post-merger integration period in the life of an organization from the human dimension. This study looks at both structural and cultural facets of the information flow in the post-merger organization as they are two foundational dimensions of any human-based organizational information system. One is forced to investigate this phenomenon by way of computer simulation experiments as similar empirical data is impossible to obtain from either the real-world or fashioned from a human-subjects laboratory.

Two distinctive outcomes from this research are a set of quantitative models that estimate features of the integration process and number of fresh perspectives on the behavioral dynamics of the post-merger integration dynamics. The models provide practitioners and scholars simple tools for making science-based forecasts of the organizational-level outcome in real-world merger scenarios. Each of the three articles presents specific research questions and propositions that drive the experiment in the article.

The remaining parts of this report are comprised of: Chapter 2, 3, and 4, which consist of the three separate reports; Chapter 5, which offers combined quantitative results; Chapter 6 containing combined qualitative results; and Chapters 7 and 8, providing the conclusions and references, respectively. This report concludes with four appendix sections (A to D). 



\section{CHAPTER 2: STUDY ONE}

\section{Post-Merger Knowledge Transfer: Examining Antecedent Structural Complexity}

\subsection{Abstract}

This paper examines the effect of prior characteristics of two merging organizations on the post-merger transfer of knowledge among organizational actors. Detailed facets of premerger organizational complexity, team complexity and infrastructure support are manipulated in controlled virtual experiments that simulate the profile of various merger scenarios. The task-specific, knowledge transfer rate of the newly merged organization is examined with regard to these antecedent control variables. Agent-based computer simulation experiments are run controlling for various structural complexity components. The organizational actors are social and are communicative. Knowledge facts are exchanged thought their interactions with other individual agents. These facts are essential for accurately performing tasks that are accomplished by individuals, work-groups, or cooperative-teams. These virtual experiments are executed in the context of an organizational merger, where two previously separate groups must now integrate into interrelated individuals and interconnected teams to perform the same tasks, but within a vastly different social and intellectual structure. The scenario for these experiments is a horizontal merger. This study investigates the postmerger integration through the lens of different metrics that capture the integration outcomes according to the levels of knowledge being held by individuals and teams assigned to specific tasks. The experiments show that each factor varies in the amount of influence each has on the transfer rate. Moreover, the combination of these factors has strong predictive power to quantitatively forecast the task-knowledge evolution in merging organizations, which can contribute to developing high-level, organization-performance projections. This study has immediate relevance to future post-merger integration research and practice by providing a theoretic and quantitative model useful for rationally estimating the post-merger knowledge transfer within a specific merger scenario. Five conclusions are reached and presented in the 
discussion. The conclusions advance the knowledge about organizational behavior during the post-merger integration period of a merger event. Moreover, they provide a baseline for an integration manager or practitioner to use as a guide to designing integration strategies and interventions.

\subsection{Introduction}

Truly-beneficial mergers--and acquisitions--(M\&A), those deemed to be an eventual success, are infrequent, and therefore the promise of a successful merger eludes many (Harrison, 2007); failure estimates have ranged starting from 50\% (Porter, 1987) upwards to $80 \%$ or more (Marks \& Mirvis, 2001). While market growth is often the primary objective or a raison d'être of a corporate merger, the deals frequently are a manifestation of an organizational strategy in which the merger is actuality an accumulation of additional human resources, i.e., the various skills and knowledge, of another organization (Lin, Hung, \& Li, 2006). In mergers with this human-asset accumulation as the driver for the combination, management focus on the human resource management (HRM) risk is essential (Bryson, 2003) and is critical to merger success. One influential measure of success is the ability of management to successfully integrate the knowledge-base among the employees.

This study focuses on such knowledge transfer in the post-merger organization. The attention is on the task-specific knowledge unique to the organization and that is essential for workers(s) to complete their assigned task(s). Since every organization has specific knowledge unique to itself, for the post-merger organization to fully integrate, this knowledge must be diffused to others in the newly combined entity. The general concepts of organization complexity, team complexity, and infrastructure support, with respect to task-knowledge transfer are employed in the formulation of the prevailing post-merger research question:

What are the directional effects of the initial differences between two organizations, Alpha and Beta, on fulfilled task-knowledge transfer levels in the combined organization, NewCo, with respect to the relative degree of their (a) organization complexity, (b) team complexity, and (c) infrastructure support? 


\subsubsection{Organization Complexity}

Organization complexity can be construed as the number of divisions, teams, or workgroups that subsume an organization. The greater the number of these subunits, the more coordination is likely to be necessary (and at several levels) and must therefore occur for the organization to perform its organization-level functions, i.e., complete the production of products and delivery of services. The greater number of these organizational subunits and thus the larger the number of possible cross-unit relationship, more complex an organization is; there are therefore many more coordination-related breakpoints that can cause failure, or opportunistically perhaps, create opportunities for greater efficiencies.

\subsubsection{Proposition 1}

The complexity of the initial organizations negatively affects the task-knowledge transfer level of the combined organization.

\subsubsection{Team Complexity}

Team complexity can also be realized by the number of individual workers (employees, associates, members, etc) there are in one of these work units. The more members, the more human interaction and coordination occur within the group, which competes with the non-group members of communications bandwidth. By interacting with proportionally more members of the same work unit, the more likely it is that task-knowledge will be exchanged; however, given a greater number of work-group members, the probability that a specific task-knowledge concept that is of value to the receiver is transmitted is therefore reduced. This local effect is expected to overbear the dilution from non-same workgroup members because of the effect of a preference that an actor would communicate with another that is more similar to oneself. 


\subsubsection{Proposition 2}

The complexity of teams in the initial organizations negatively affects the taskknowledge transfer level of the combined organization.

\subsubsection{Infrastructure Support}

The level of infrastructure support in an organization is relevant for an investigation into task-knowledge transfer because having more technology in an organization can reduce the amount of task-knowledge that must be passed through person-to-person interaction for the completion of a given task. Instead, this necessary task-knowledge is stored in technological systems, such as employee manuals, web pages, posters on the walls, and computer-based training programs and databases. The exchange of existing task-knowledge from one organization to members of another, when embedded in technology, is void of many human issues that are not removed in a human-to-human knowledge exchange.

\subsubsection{Proposition 3}

Infrastructure support within the initial organizations positively affects the taskknowledge transfer rate of the combined organization.

\subsection{Research Design}

To explore these propositions and construct a computational model for the research question, an agent-based computer simulation methodology is employed. The limited scope of real-world case and laboratory studies cannot provide the fidelity in the data that a virtual experiment can provide with relative ease. The design of the experiment is described below by presenting the general case scenario, the various characteristics of the software used, and the particulars to the designed experiment.

\subsubsection{Simulated Case Scenarios}

This simulation is based on a realistic organizational behavior and merger script. The scenario is much like a typical real-world case found in academic case-studies and is the script 
to which the computer simulation is guided and from which the virtual experiments are designed. Two independently conceived organizations, Alpha and Beta, are thrust into a merger to form NewCo. Alpha and Beta are envisioned to provide similar products and services that complete in the same market and thus it is a horizontal merger. Alpha (the acquirer) is seeking to gain a larger share of its market and hopes to gain economies of scale through various synergies by combining with Beta. Cost-cutting is not part of NewCo's forward strategy; moreover, workforce redundancies are not of concern and therefore layoffs not part of the virtual script.

At the start of the virtual experiment, when Alpha and Beta are actually merged, the components of each are commingled at the team-level task under the NewCo umbrella. Consequently NewCo consists entirely of the pre-merger characteristics and pre-existing social networks of Alpha and Beta. This situates NewCo as a possibly eclectic mixture of socio-technical sub-networks that have become intermingled and must now interact according to the proscribed social behavior theories contained in the computational model, i.e., Construct.

\subsubsection{Construct Software}

The simulation software used is based on CONSTRUCT, which is a validated model of social behavior that has been used in numerous studies and is a powerful model for simulating organization behavior. Construct is grounded in the Carnegie School of social theory: people interact with one another within a complex network of social-technical relationships and exchange personally-held information, or knowledge, between themselves. CONSTRUCT, an agent-based model, also incorporates the notion of transactive memory in the actor-to-actor exchange. This is to say that people can transmit to their exchange partner, their knowledge of another person knowing of some particular fact or information. 


\subsubsection{The Representation Model}

The representational model implemented within Construct is based on the metanetwork construct (Carley, 2002a, 2002b), which is a primary feature of Dynamic Network Analysis (Carley, 2003). Numerous networks are formed from three basic entity types: actors, knowledge and tasks. Broadly, the representation model is consistent with the PCANS model (Krackhardt \& Carley, 1998). From the three entity types, four types of networks are utilized. The first is the social network, which is an actor-to-actor relationship. The second is an assignment network consisting of actor-to-task relationships. The third is a task-to-knowledge network which represents the concepts that are necessary to perform each task. The fourth is the knowledge network which is an agent-to-task network representing what concepts each agent knows.

\subsubsection{The Behavioral Model}

The behavioral model that controls how agents operate is an operationalization of the Construct model (Carley, 1991). Construct theory has its underpinnings in social theories such as symbolic interactionism (Blumer, 1969), structural interactionism (Stryker, 1980), and structural differentiation theory (Blau, 1970). In the Construct model, the informationprocessing perspective of the Carnegie School (Simon, 1957; March \& Simon, 1958; Cyert \& March, 1963), i.e., people are information seeking, is integrated with social-informationprocessing theory (Salancik \& Pfeffer, 1978; Rice \& Aydin, 1991), i.e., what knowledge a person has is dependent on what information they have access to via their social network. Agents purposefully interact through complex discourse (Dooley, Corman, McPhee, \& Kuhn, 2003) and social interaction (Carley, 1986) to exchange knowledge and frequently seek out others who have knowledge that they do not yet hold, and are sought out by others seeking their knowledge. 


\subsubsection{The Merger Transaction Scenario}

An organizational merger transaction is a single point-in-time event that involves combining the various socio-technical networks of Alpha and Beta to form NewCo. The respective organizational teams from Alpha and Beta are paired and combined at the start of the simulation run. Once NewCo is setup, as a facet of the simulation initialization process, the behavioral model described above is in full authority of the behaviors occurring within the simulation. This simulation does not differentiate any behaviors between running in an ordinary or a post-merger situation.

\subsubsection{Control Variables}

Organization Complexity (OC) is a construct that can be partially manifested by the number of sub-parts of an organization, which implies the amount of complex interactions that are designed into the organizational structure. For the purposes here, the number of subparts in the organization is manifest as the number of teams in the organization. For this study, the underlying variable for $\mathrm{OC}$, teams/org can take on a value of 1, 5, 10, which are arbitrary settings as real-world organizations can vary greatly in the number of teams, departments, silos, etc. A value of 1 is reasoned to allow for these experiments to address an organizational merger between two organizations that do not have any sub-parts; for example, two teams merging. Unlike most other variables in this experiment, the teams/org variable is the same for Alpha and Beta; structural similarity has been found to be relevant in intra-firm knowledge transfer (Mowery, Oxley, Silverman, 1996) and this identity feature is necessary because the integration of NewCo is consummated at the team level. This is to say that at the time of the merger event, the teams in Alpha are paired 1-to-1 with teams in Beta, resulting in the same teams in NewCo, just with more members. This phenomenon of matching teams is consistent with a horizontal merger, whereas to organizations in same businesses merge

Team Complexity (TC) is a construct, as with $\mathrm{OC}$, that is a function of the number team members and is indicative of the number of interactions within a team for the team to function. This can indicate the amount of coordination and communications (among 
individuals) that must take place for the team to perform its assigned tasks and is an important determinant of complexity (Carroll \& Burton, 2000). In a manner comparable to Axelrod's (1986) meta-norms virtual experiments, Beta's team size varies relative to the fixed Alpha's team size; herein at a factor of 0.5 times, 1.0 times, or 2.0 times (Cording, Christmann \& King, 2008). It therefore follows that the team size for NewCo will be Alpha's team size plus Beta's team size since the teams are combined at the time of the merger (as mentioned in the discussion on the $\mathrm{OC}$ construct). Furthermore, the total size of an organization-the number of workers in its workforce-is determined by multiplying the values for OC and TC for a given organization.

Infrastructure Support (IS) is a construct that indicates the level of infrastructure support that each team has available to it that aids it in performing its assigned tasks. An appropriate fit of technology to the needs of the task and team is important for the group's performance (Keller, 1994). This construct is represented by the amount of knowledge concepts necessary for the team to perform their assigned task. The less infrastructure support available to the team the more relative knowledge the team will require to perform its assigned tasks. This also can be thought of as cognitive demand required of the team. The notion of knowledge per team should be thought of as a relative differential between two teams. Considering that two teams being combined perform the same tasks, thus must hold the same knowledge to perform the task, for example two teams of accountants. However, one team has access to a sophisticated accounting system that can create targeted reports that answer a specific question. On the other hand, the other team does not have the benefit of such technology and therefore, to answer the exact same question, needs to have knowledge of what data is relevant and important, and how to combine facts to construct a reply. The first team simply has an individual push a button. It is knowledge concepts such as this that IS represents. The less knowledge needed to perform the same task, is generally considered more efficient and better for the team and broader organization. This construct has a fixed parameter setting for Alpha and a variable setting for Beta because it is the relative differences between Alpha and Beta that are being modeled in this experiment. 


\subsubsection{Factorial Design}

The five control variables each have their value determined from a set of three possible values. From this, there are 243 individual experimental cells $(3 \times 3 \times 3 \times 3 \times 3)$. A single outcome variable is computed at the end of each simulation time period. Each independent run (each experimental cell) of the simulation is stopped after running for 1000 time periods. These constructs, variables and parameters are summarized in Table 2-1.

Table 2-1. Control Variables

\begin{tabular}{cc}
\hline Control Variable & Values \\
\hline ORGANIZATION COMPLEXITY & \\
Teams per organization (both) & $2,6,10$ \\
TEAM COMPLEXITY & \\
Agents per team Alpha & $4,10,16$ \\
Agents Per Team ratio, Beta & $0.5,10 ., 2.0$ \\
INFRASTRUCTURE SUPPORT & \\
Knowledge per team & $20,30,50$ \\
Knowledge per team ratio, Beta & $0.5,1.0,2.0$ \\
\hline
\end{tabular}

\subsection{Results}

There are five control variables and one response variable in this experiment; all are continuous variables, with possible values greater than or equal to zero. The control variables include: (a) the number of teams per organization (value is the same for Alpha and for Beta), which is a variable making up organizational complexity, (b) the number of agents per team for Alpha and the relative setting for Beta, which makes up Team Complexity, and (c) the number of organization-specific knowledge concepts per team for Alpha and the relative setting for Beta, which makes up Infrastructure Support. The response variable for this experiment is the task-knowledge transfer level.

Figure 2-1 shows the difference of the task-knowledge level across the three values for the Organization complexity variable. The average task-knowledge level at time period 999 is $89 \%, 72 \%$, and $66 \%$ for the three settings. 


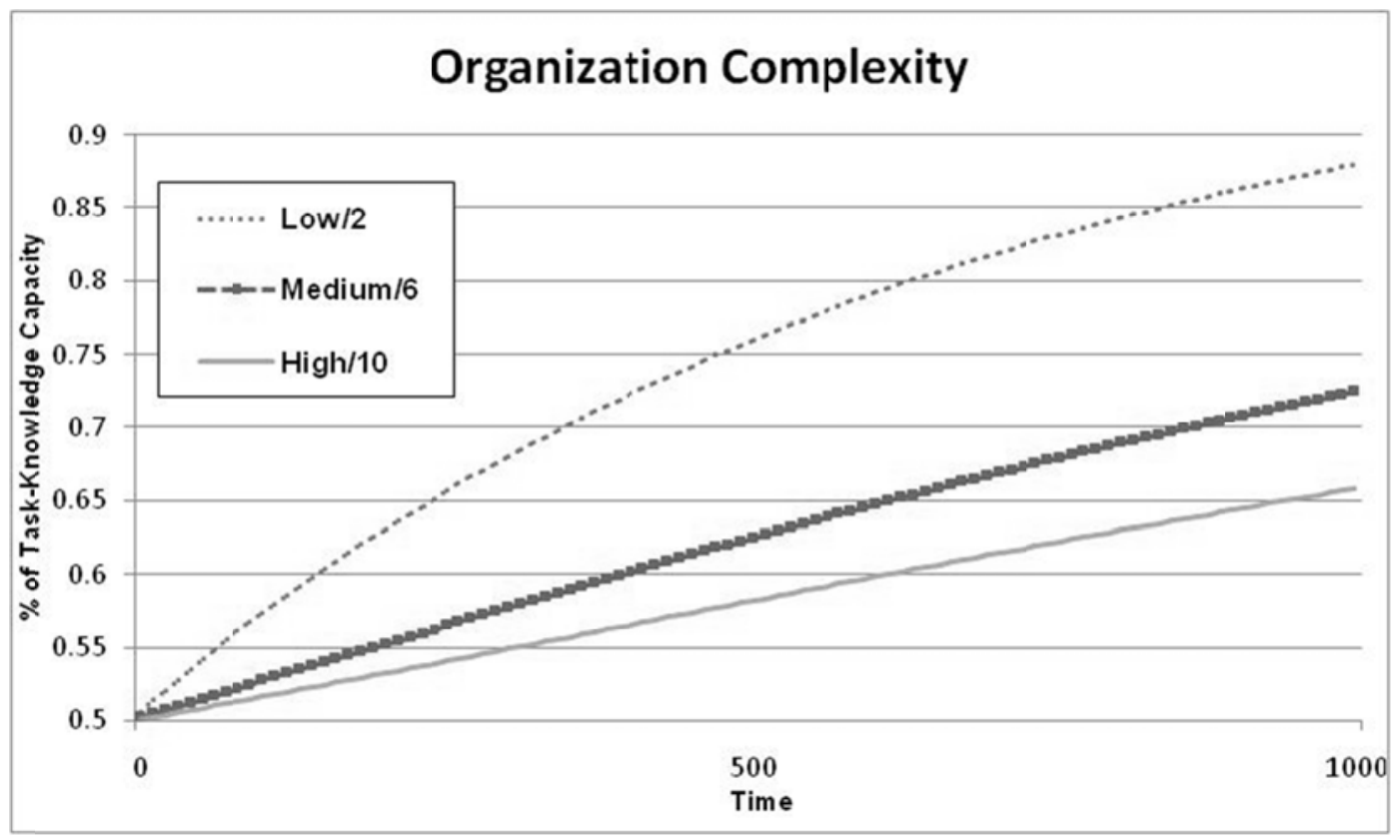

Figure 2-1. Comparison of average values for Organization Complexity variable over time.

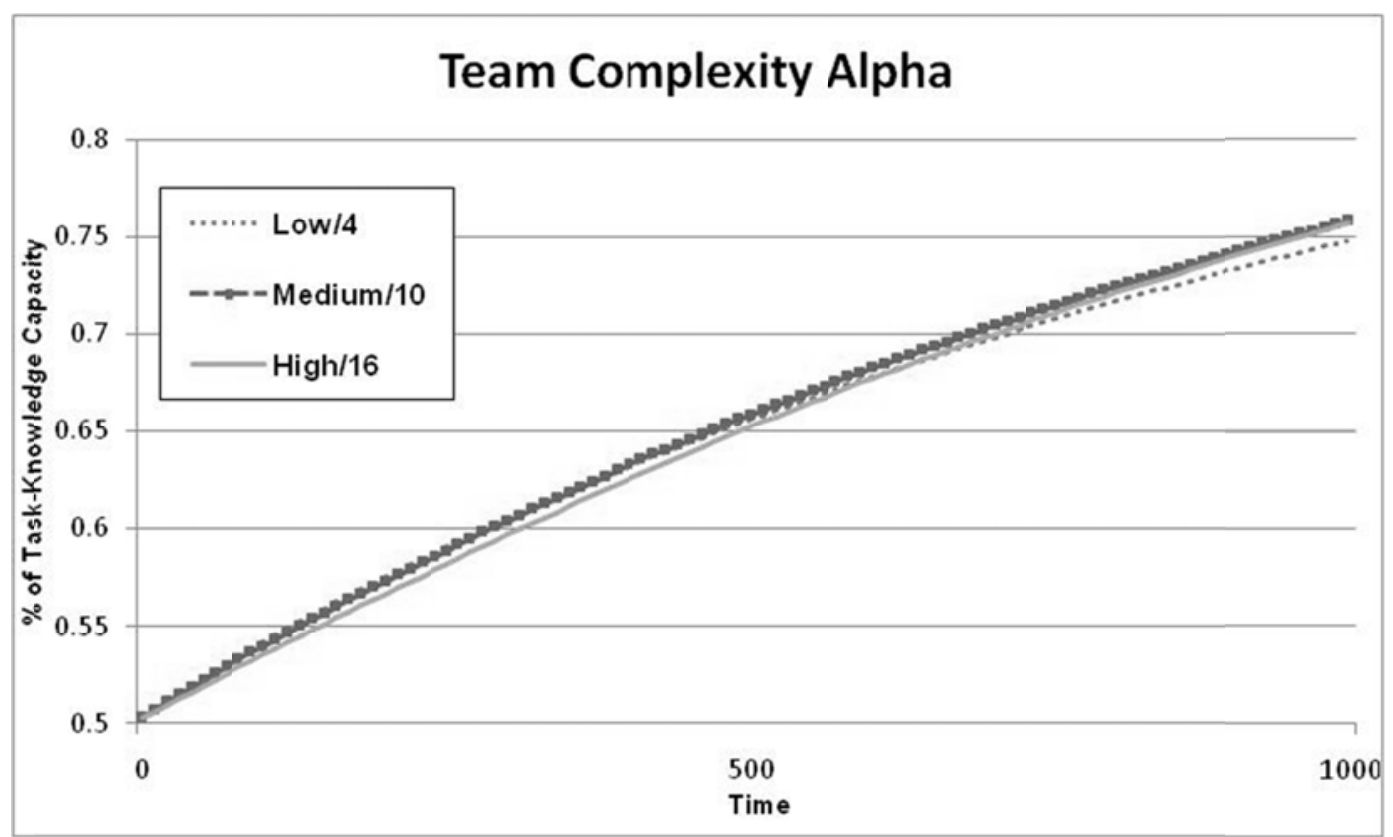

Figure 2-2. Comparison of average values for Team Complexity Alpha variable over time. 


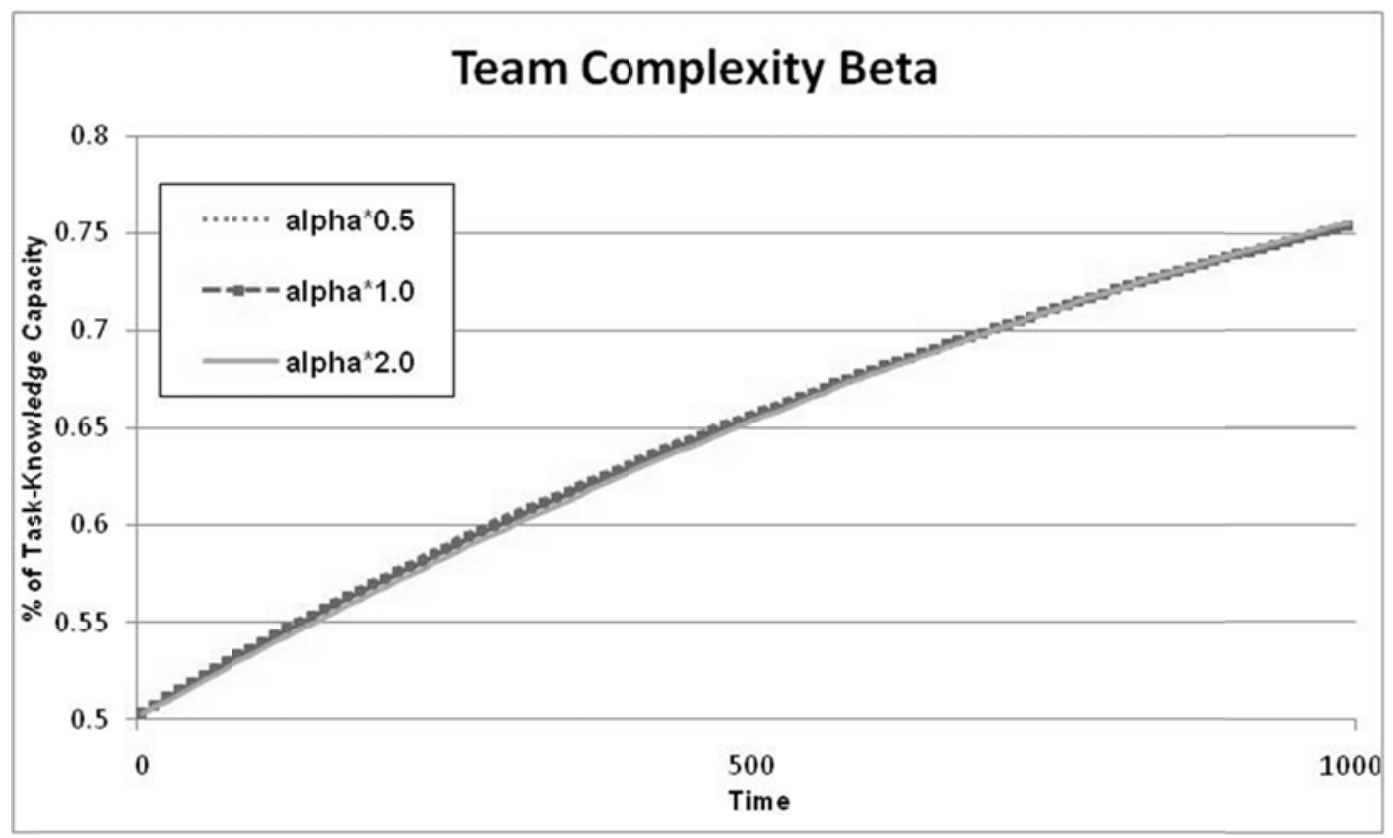

Figure 2-3. Comparison of average values for Team Complexity Beta variable over time.

Figure 2-2 shows the difference of the task-knowledge level across the three values for the Team Complexity Alpha variable. The average task-knowledge level at time period 999 is $75 \%, 76 \%$, and $76 \%$ for the three settings. Figure $2-3$ shows the difference of the taskknowledge level across the three values for the Team Complexity Beta variable. The average task-knowledge level at time period 999 is $75.3 \%, 75.4 \%$, and $75.5 \%$ for the three settings.

Figure 2-4 shows the difference of the task-knowledge level across the three values for the Infrastructure Support Alpha variable. The average task-knowledge level at time period 999 is $79 \%, 76 \%$, and $71 \%$ for the three settings. Figure $2-5$ shows the difference of the taskknowledge level across the three values for the Infrastructure Support Beta variable. The average task-knowledge level at time period 999 is $78 \%, 76 \%$, and $72 \%$ for the three settings. 


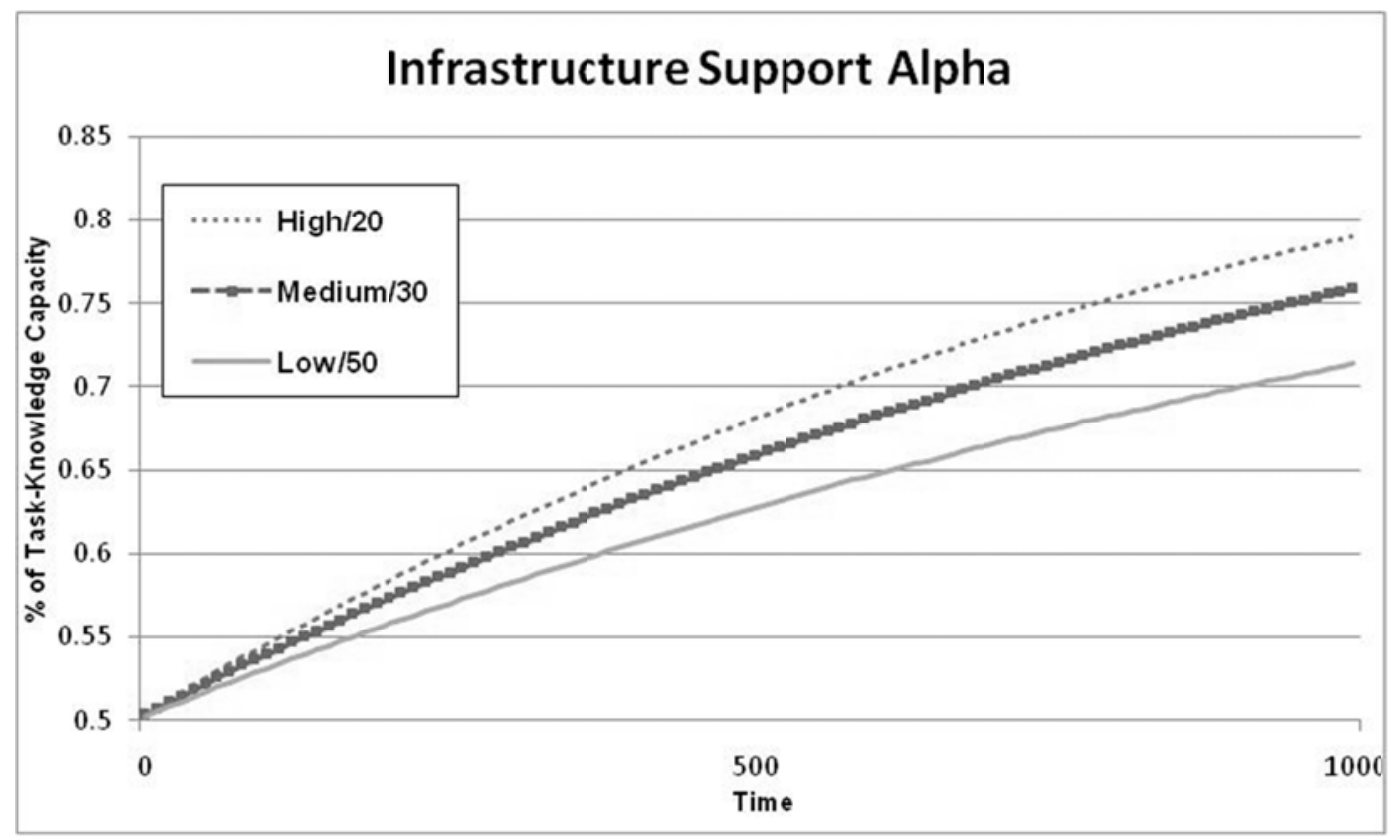

Figure 2-4. Comparison of average values for Infrastructure Support Alpha variable over time.

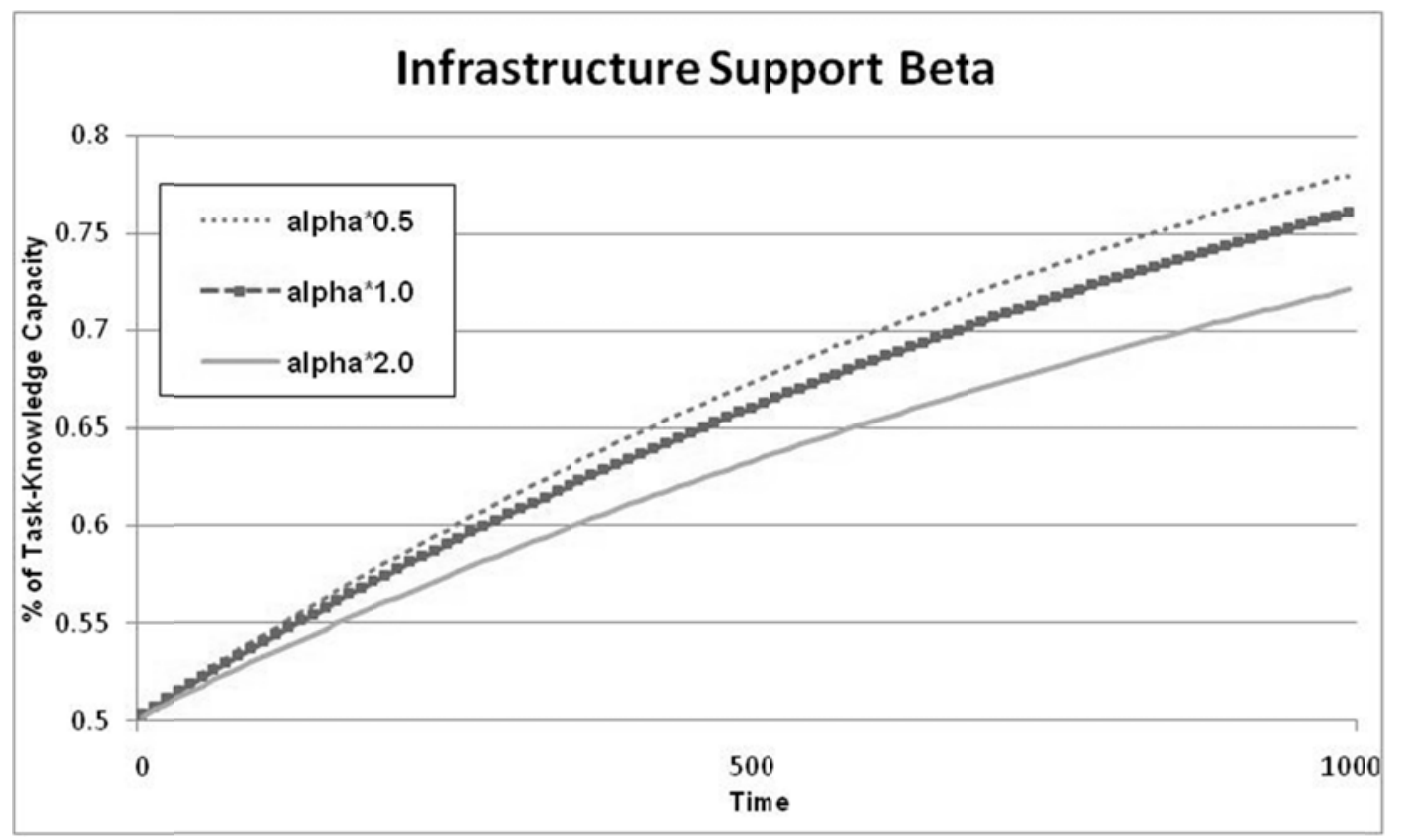

Figure 2-5. Comparison of average values for Infrastructure Support Beta variable over time.

Table 2-2 shows the regression results of the relationship between the control variables and task-knowledge transfer. The model uses time $(t)$ as a parameter since the 
regression is run on a vector of data that consists of taking a snapshot of the outcome variable every 10 time periods. The control variables do not change during the simulation run. The resulting regression model explains $88 \%\left(R^{2}=0.88\right)$ of the variance in task-knowledge transfer. The each beta coefficient in the regression model is statistically significant and indicates that each control variable has a negative effect on task-knowledge transfer. As the number of teams (organization complexity) in alpha and beta increases the expected taskknowledge transfer level will decrease. Consistent with the effect of organization complexity, as the number of actors in these teams increase (team complexity), task-knowledge transfer will decrease. Moreover, the number of knowledge concepts per team has a negative (thus infrastructure support has a positive), the effect on task-knowledge transfer.

Table 2-2. Relationship between pre-existing factors and Knowledge Transfer: OLS Estimation.

\begin{tabular}{ll}
\hline & Coefficient Estimate \\
\hline Intercept & $0.721698 * * *(0.000111)$ \\
time & $0.000251 * *(0)$ \\
& \\
Organizational Complexity & \\
$\quad$ Teams Per Org & $-0.019508 * * *(0.000007)$ \\
Team Complexity & \\
$\quad$ Agents per team alpha & $-0.000157 * *(0.000004)$ \\
$\quad$ Agents per team ratio Beta & $-0.002008 * * *(0.000035)$ \\
Infrastructure Support & \\
$\quad$ Knowledge concepts per team Alpha & $-0.001581 * * *(0.000002)$ \\
$\quad$ Knowledge concepts per team ratio Beta & $-0.024652 * * *(0.000035)$ \\
\hline
\end{tabular}

$R^{2}=0.88 ; *, * *, * * *$ indicate significance at the two-tailed $(0.10,0.05$, and 0.01$)$ levels, respectively. Standard errors are in parentheses.

\subsection{Discussion}

This study examines the effect of prior characteristics of two merging organization on the post-merger task-knowledge transfer. Virtual experiments are conducted that manipulate facets of pre-merger organizations; these include: organizational complexity, team complexity and infrastructure support. Details of the experimental design and results of the experiments are presented above. 


\subsubsection{Quantitative Findings}

Looking at the results broadly, it becomes clear that the task-outcome variable, taskknowledge characteristically either stays the same or monotonically increases over time; this is a trait of the model implemented in this experiment. For some instances of the experiments, the rate of change if the outcome variable is initially relatively high and gradually approaches zero over time. This indicates that on the outset, there is a great deal of new knowledge exchanges that over time diminishes as the amount of new knowledge left to exchange approaches zero. This is an artifact of the circumstance that initially there is a great deal of difference in the knowledge held by actors initially, which is reduced gradually as this difference in knowledge-held is reduced as knowledge diffuses in the organization.

Turning to the outcome variables specific to each experiment where a single control variable is altered, first is organizational complexity. Initially, the rate of knowledge transfer is much greater in the lower organization-complexity scenario. That is, initially the more complex an organization, the lower this immediate rate, but then later, becomes larger than the less complex organization. Over time, this rate becomes about the same no matter the level of complexity and the rate of change ultimately reaches zero.

Looking at the results for Team complexity, for the alpha variable it appears that there is very little statistical effect from varying the level of team complexity. The trajectory of the change rate for Team complexity does appear to be characteristically similar to that of Organization complexity, but the time scale is greatly shortened. This is to say that the less complexity the higher the change rate initially, and then crosses over to a lower rate over time. For the team complexity beta variable, it appears that there is little statistical effect from varying the level of team complexity for beta. The trajectory of the change rate for team complexity does appear to be characteristically similar to that of organizational complexity, but the time scale is greatly reduced. This is to say that the less complexity the higher the change rate initially and them crosses over to a lower rate over time. 
The results for Infrastructure support suggest that the variable is relevant. For the Infrastructure support alpha variable, the lower level of infrastructure support show a lower level of the outcome variable and also a lower rate of change. The same holds true for the infrastructure support beta variable.

Looking to the regression results: the formulation of the experiment outcome is a linear regression model, i.e.,

Task-Knowledge level $=0.7217+0.0003 * b_{1}-0.0200 * b_{2}-0.0002 * b_{3}-0.0020^{*} b_{4^{-}}-0.0016^{*} b_{5^{-}}$ $0.0247 * b_{6}$

$$
\text { Where } \begin{aligned}
b_{1} & =\text { time } \\
b_{2} & =\text { organization complexity } \\
b_{3} & =\text { agents per team alpha } \\
b_{4} & =\text { agent per team ratio aloha to beta } \\
b_{5} & =\text { knowledge concepts per team alpha } \\
b_{6} & =\text { knowledge concepts per team ratio alpha to beta }
\end{aligned}
$$

and Task-Knowledge Level is a continuous value between 0.0 and 1.0, inclusive. This model has an $R^{2}$ of $88 \%$, which is high.

The regression equation is consistent with and confirms the exploratory visual analysis. These results are not unexpected; they coincide with the three proposition present in the introduction: (1) The complexity of the initial organizations negatively affects the taskknowledge transfer rate of the combined organization, (2) the complexity of teams in the initial organizations positively affects the task-knowledge transfer rate of the combined organization, and (3) infrastructure support within the initial organizations positively affects the task-knowledge transfer rate of the combined organization.

What is novel from these results is not the sign of each factor, but that specific quantitative values can now be assigned to each. First, looking at the standardize coefficient for each term, the factors can now be rank-ordered from most important factor to least: (1) number of teams in the organization, (2) the infrastructure support level, (3) the relative amount of structural support in one organization relative to the other, (4) the size of the teams, and (5) the relative size of the teams in one organization relative to the other. 
Using the derived regression equation, now a reasonably estimate the level of taskknowledge in the merged organization can be determined. For example, an alpha (and beta) organization with 2 work units, with 10 employees in each unit, and 100 task-knowledge concepts per team, merging with a beta organization also with 2 work units, twice as many employees per team, and twice as much infrastructure support (50\% of the task-knowledge concepts per team) would have a task-knowledge level of 0.697 after 999 simulation periods.

From this regression equation a response to the research question set forth at the conception of this study can be determined. The research question being: what are the quantifiable effects of the initial differences between Alpha and Beta on Task-Knowledge Transfer in NewCo, with respect to the relative degrees of their organization complexity, team complexity, and infrastructure support?

The regression equation derived from this experiment can be utilized for estimating the task-knowledge exchange rate well before the consummation of a merger. There is more to be done, but this simplistic mode is a step forward in the development of a stronger more powered quantitative model. Future work involves examining other aspects of the organization with a similar research design in order to construct an idealized fuller and richer quantitative model.

\subsubsection{Conclusions}

Five pertinent qualitative conclusions can be derived from the quantitative results of this experiment:

1. Organizations with lower organization-level will make greater, more noticeable progress in task-knowledge diffusion early-on in the post-merger integration period.

2. Team-level complexity has a dynamic characteristically similar to that of organization-level complexity.

3. The relative difference in team-level complexity between the two original organizations has minimal effect on the task-knowledge transfer rate. 
4. The level of infrastructure support positively affects the speed to which the organization reaches its task-knowledge saturation point.

5. The relative difference in infrastructure support between the two original organizations has minimal effect early on in the integration process, but becomes more prominent in later stages of the process.

These results advocate that an integration manager would be well-served to assess the organizational complexity, team complexity and infrastructure support of the two merging organizations when designing a post-merger integration strategy. Herein, these factors have been shown to affect the trajectory of the task-knowledge diffusion in the post-merger organization. This study provides a base-line indication of what may transpire if the merged organization is left to integration on its own without oversight. While this is most often an unusual scenario, it does indeed happen in some cases, but is usually an ill-advised strategy for integration. 


\section{CHAPTER 3: STUDY TWO}

\section{Post-Merger Integration: Effects of Task Structure and Motivation to Exchange Information on Organization Performance}

\subsection{Abstract}

This study focuses on the relationship of structure and motivation on organization performance during the integration period following an organizational merger. Computational modeling and computer simulation is applied to generate an extensive dataset for use in constructing quantitative models of these relations. To evaluate organization performance, the amount of information held by workers, relative to the amount of specific information necessary to perform assigned tasks is measured. The extent to which task-related information is available in a group is indicative of the performance of its members; subsequently leading to the level of performance for the entire work-group and the organization, in the aggregate. The structure of the work tasks from two perspectives is studied: (a) the degree to which employees specialize, and (b) the level of team interdependence. Likewise, motivation toward task-information exchange is also studied from two perspectives, specifically: (a) employees' willingness to seek information, and (b) employees' willingness to share information. Following the execution of the virtual experiments, two quantitative models are constructed, which can be used for quantitative forecasting, and developing qualitative findings that can be applied to practice, i.e.: (a) integration managers may safely not be overly concerned about motivating the member of the acquired organization to share information with members of the acquiring organization, (b) an organization with lots of interdependent work-teams may require less attention during the post-merger integration than an organization made up of unconnected work groups, and (c) it may be just as effective to focus on information exchange only to a point of critical mass rather than attempting to attend to all members of the combined organization; the outcome may be just as positive. Finally, it can be surmised that structural characteristics may be more consequential than motivational characteristics in realworld mergers. This study has immediate relevance to post-merger integration research and 
practice by providing a precise quantitative models and qualitative appraisals, both advantageous for estimating post-merger performance and formulating post-merger integration strategy.

\subsection{Introduction}

Organizational mergers -- and other forms of strategic combinations, such as acquisitions, alliances and joint-ventures-- (M\&A) are commonplace, as business growth is expected and is expansion is part of the human yearning. M\&A involves people: It has been estimated that in a single year, $10 \%$ of the American workforce is directly affected by commercial M\&A activity (Buono \& Bowditch, 1989). Such an estimate could even be considered quite conservative (Schweiger \& Walsh, 1990). Indeed, at hyperactive periods in the economic cycle, barely a month goes by without at least one announcement of a massive corporate M\&A deal (Bruner, 2005), which can often involve a hundred thousand employees directly. No matter the size or setting, organizational mergers are ubiquitous; may they comprise corporate entities, family enterprises, government agencies, NGOs, educational institutions, church groups, or simply little-league baseball teams.

Successful mergers - those deemed to have an eventual benefit relative to a nonmerger course-are quite scarce. Achieving any level of post-merger integration success eludes most attempts (Harrison, 2007). Estimates of success rates are dire. Optimists proclaim as much as a $50 \%$ success rate (Porter, 1987), while others assert that the success rate may be as low as $20 \%$ (Marks \& Mirvis, 2001). Strategically, it is common that an M\&A deal is struck for the purpose of adding highly-valued human resources. These human assets are naturally accompanied by sought-after skills and knowledge at the individual, group and organizational level (Lin, 2006). Ordinarily, a management focus on these human resource assets and any intrinsic liabilities is vital (Bryson, 2003).

Effectively combining the human resources, including their embedded knowledge, is critical to establishing immediate post-merger and longer-term success. One approach to this task is to concentrate on the information diffusion throughout the new organization. Research indicates that knowledge and information exchange processes and dynamics during the post- 
merger integration period differ from normal times (Bresman, Birkinshaw, \& Nobel, 1999). It may be this information exchange aspect of the human resources that may be the underlying cause of integration and M\&A failure.

This research is shaped by recognizing the immense consequence of these human resources and the relevance of the informational aspect of their activities. Consequently, for this study I wholly honor the theoretic and practical, information-based view of the organization. This implies that to evaluate the effectiveness of an organization one assesses the dynamics of the information flow in the organization. A goal-seeking organization is considered effective to the extent that the information necessary for performing a task is held by those persons performing that task. Subsequently, M\&A is deemed successful if the newly formed organization is able to get information necessary to accomplish tasks to those that are performing them. This research is future grounded on the notion that specific information necessary to perform some tasks deviates from legacy requirements during organizational disruption, particularly if the entire organization changes as a result of M\&A.

This study looks at both structural and motivation facets of the information flow in the post-merger organization as they are two foundational dimensions of any human-based organizational information system. This requires investigation of this phenomenon to be conducted by way of computer simulation experiments as empirical data is practically impossible to obtain from either the real-world or fashioned from a human-subjects laboratory.

The remaining sections of this report provide: (a) First Perspectives that are important to understanding the foundations, features and limitations designed into this simulation-based study, (b) the cornerstone research questions this study is concentrated upon, (c) the methodology and design of the virtual experiments, (d) the quantitative results of the experiments, and (e) a discussion of the findings, how they can be applied and the inherent limitations of this study. Finally, an Appendix Section that details the underlying computational model that is depended upon, and that is both the theoretic foundation of how 
an organization operates in our virtual environment and how this virtual world aligns with the real world to the extent to which is modeled herein.

\subsubsection{First Perspectives}

Before proceeding into a phenomenological discussion about post-merger integration, critical first perspectives that are essential to understanding this research are presented. Herein, an agent-based model for the computer simulation is employed. A power feature of the agent-based methodology is that the modeler can purposefully craft the behavioral rules of the agent/actors under a highly-selective and purposeful basis (Note: Here, we'll refer to agents as actors). Furthermore, the modeler also has complete control over the environment within which the actors perform their activities following the behavioral rules, which allows the simulations to be highly specific toward the precise situation being modeled. The general foundations and generic details of the agent-based, computational model used herein are provided later in the Methods Section; however, herein the extensive capacities of the agentbased paradigm that are have implemented, consisting of several key features are employed. These critical, phenomena-related features are introduced and expounded below.

\subsubsection{Information Processing Paradigm}

Conceptually, an organization is viewed as as social construct designed for an instrument fashioned to handling information; abstractly, a machine a machine, perhaps. Within its boundaries, an organization is essentially an information processing system with complex internal dynamics. Subscribing to the Carnegie School of Organizational Theory (Cyert \& March, 1963; March \& Simon, 1958; Simon, 1957), the organization's informationprocessing capability is concerned a critical underpinning for decision making and performance. There have been many attempts at precisely defining the term information without settlement on any concrete consensus (Case, 2007); since this study is only abstractly modeling the real-world, the meaning of the term is preserved, as remaining highly-abstract. For example, Chaffee's (1991, p. 9) suggestion that information is "anything that exists psychologically for a person" as a possible guidepost to how it is idealize as information 
within the confines of this study. Also note that the term knowledge will often be used in the contextual discussion of this paper; readers should make no distinction between the two terms and should remain rather flexible and become unattached in envisioning the boundaries of this central construct.

\subsubsection{Information Exchange}

Considering the information processing facet of an organization (Galbraith, 1977), actors interact with one another as a matter of routine, i.e., they socialize. In the course of this repetitive socialization process, actors purposefully exchange information with one another, intentionally or unintentionally. They pass information either through direct personto-person communications or through more indirect and contemporary forms, such as electronic emails and broadcast media (Dooley \& Corman, 2004). Keeping with our abstract understanding for information, the process by which information is exchanged with others, e.g., verbally, visually, even subconsciously, should also be held as being highly abstract.

The organizational processes supportive of information exchange, or knowledge transfer, are "a basis for competitive advantage" (Argote \& Ingram, 2000, p. 150; Zander \& Kogut, 1995). Consequently, information exchange is a predominant concern of management during post-merger integration, since mergers are primarily consummated for the purpose of improving a firm's competitive advantage (Bruner, 2004). The exchange of information among the members of an organization is critical to individual and organizational performance from both social and actionable perspectives, and supports central concepts such as organizational learning (Argote, Gruenfeld, \& Naquin, 2000). In the usual sense, knowledge transfer refers to the transferal of existing "reservoirs of knowledge" (Argote \& Ingram, 2000, p. 153) across organizationally-bounded groups; however, this convention is amended to refer to such transfer occurring "within a newly-combined group" rather than across. Pre-merger groups will hold unique reservoirs of knowledge such that the a subset of the constituent elements--e.g., the tactic and extrinsic skills, contextual practices, routines, etc--, are particular and specific to their group; this holds nonetheless, even if two groups 
perform the same functional tasks; every organization has unique variances in its work processes, procedures, etc.

In the special setting brought about during post-merger integration, effective knowledge transfer is paramount. In order to realize the newly expected post-merger performance level, the previous disjoint knowledge reservoirs must ultimately meld into a single pool of shared knowledge - a post-merger knowledge reservoir united within a single group. As part of the pre-merger planning, finances can easily be combined in an orderly and predictive matter; however, in the realm of an organization's knowledge-base, this integration of human knowledge is more of a complex and dynamic-perhaps chaotic-process (Szulanski, 2000), as opposed to the obviously-important but comparatively less-dimensional legal and accounting transactional events.

\subsubsection{Factors affecting the Transfer of Knowledge}

Operative organizational knowledge transfer is a hard to accomplish (Argote, 1999; Szulanski, 2000) and is influenced by numerous factors (Becker \& Knudsen, 2006) and subprocesses, e.g., practices such as actor rotation between groups can facilitate transfer under the right conditions (Almeida \& Kogut, 1999; Argote, 1999). There is plentiful awareness and conceptual models of this intra-firm transfer process in the literature, e.g., Schlegelmilch and Chini (2003). Knowledge transfer in an organization is a process with specific constituent stages with different factors affecting its stickiness (Szulanski, 1996, 2000). The communication among a work team is critical to the development of new ideas and knowledge (Boland \& Tenkasi, 1995). In a laboratory setting, it was established that social identity affects the transfer of knowledge (Kane, Argote, \& Levine, 2005): when an individual socially-identifies with a group (and the group likewise identifies with the individual), as social identity theory (Tajfel \& Turner, 1979, 1986) suggest as being a factor in intergroup behavior, it has been found that the individuals view of the group (and the groups view of the individual) is positive. Therefore, as derived in Kane, Argote, and Levine "individuals may feel more comfortable sharing knowledge with groups with whom then share social identity than with those that they do not..”(2005, p. 57). It has been shown that an individual whom 
has temporarily left their social group to visit another group and obtain outside knowledge and ideas, returns to find that they have less influence in their home group (Gruenfeld, Martorana, \& Fan, 2000) and that such integration is essential to knowledge flow (van Wijk, Jansen, \& Lyles, 2008; van Wijk, van den Bosch, Volberda, \& Heinhuis, 2005). Research has also found that there can be inequalities among social groups in the availability and acquisition of information (Viswanath, Kahn, Finnegan, Jr., Hertog, \& Potter, 1993).

\subsubsection{Task-Knowledge}

In this study, the attention to knowledge is solely to that which is relevant to the performance of a specific task, though other information can be vitally important, e.g. to advance group innovation (Inkpen \& Dinur, 1998). These task relevant concepts are referred to as task-knowledge. Like many other aspects of this model, exactly what constitutes a relevant concept of knowledge is highly abstract although it steadfastly is some specific information that is necessary to perform the given task expertly. For example, in a pizzamaking task, knowledge of how to toss the dough is relevant to making pizza as is knowledge of how to combine the ingredients making the pizza sauce; while, knowing how to clean an over may be useful to a pizza maker, it is not necessarily essential to being a world-class pizza maker (cleaning the oven might be abstracted as being a separate task, perhaps).

\subsubsection{Task-Knowledge and Performance}

Members of a group each bring different expertise, knowledge, and information into their group (Jackson, 1992) and the distribution of the knowledge within a group varies (Liang, 1994). The knowledge held by a group directly influences the performance of the group (Shaw 1981; Steiner 1972; Stasser \& Stewart, 1992). The distribution of the knowledge within the group can be deliberately managed (King, 1999) and greatly affects group performance (Rulke \& Galaskiewicz, 2000). How broadly the information is distributed and the amount of information shared within the group has been shown to affect the group performance (Stasser, Taylor, \& Hanna, 1989; Stasser \& Titus, 1985, 1987). Moreover, there can be complicated social dynamics occurring within a small group when it comes to 
exchanging such information (Deeker, Stokman, \& Franses, 2000). Localized knowledge common to a specific group can face natural difficulties being transferred outside of that group (Almeida \& Kogut, 1999), unless there is exchange of personnel among the group, such as within an industry, for example; in our case herein, melding human assets within a newly combined organization.

To evaluate the progress of the organization during the post-merger integration period, thus measuring performance, task-knowledge capabilities within the merged organization is focused on. The amount of knowledge that has been transferred not from a generic perspective, but from the qualitative perspective is measured. That is to say, knowledge transfer is measured for only those concepts that have value to the specific members of the organization, necessary to perform their work tasks, given the task-assignment structure of the individual work units.

\subsubsection{Knowledge Exclusivity to an Organization}

This study focuses on the knowledge transfer in the combined, post-merger organization. In particular, the focus is on the task-specific knowledge that is unique to previously detached workgroups and that has become essential to newly formed groups, such that the knowledge is essential for workers to complete a given task. In some work teams, knowing how to access information contained in the group is essential (Stasser, Vaughan, \& Stewart, 2000). Research indicates that organization change can affect knowledge transfer, and thus performance (Lin, 2000). Since each organization has specific knowledge unique to itself, for the post-merger organization to fully integrate, this knowledge must be diffused to others in the combined entity. This study intendeds to be a step toward developing a predictive capability for real-world mergers, thus it incorporates the pre-merger characteristics of the two organizations into the study design. The study aim to uncover the correlations--not necessarily causality--between the performance-relevant knowledge transfer rate in the combined organization with that of relatively easy to estimate characteristics of the two organizations. 
Furthermore, the experiment is restricted to the set of task-knowledge concepts that is mutually exclusive across the same task in Alpha and Beta. For example, in a pizza-making task, knowledge of how to toss the dough is common knowledge across two organizations and is therefore not captured in this virtual environment. However, knowledge of how to combine the special ingredients making the pizza sauce differs across organizations and is therefore deemed as a task knowledge concept in this virtual environment. Only organizational unique concepts are modeled herein; it would be meaningless to track the flow of information throughout an organization if, in fact, those that need to know a particular informational concept, already do.

\subsubsection{Research Questions \& Propositions}

\subsubsection{Structure and Motivation}

I deliberate over two overarching research questions that refer to social patterns that are known to affect information exchange within an organization (see Bresnen, 2003): (a) structure (Cowan \& Jonard, 2004; Reagans \& McEvily, 2003; Wickesberg, 1968), and (b) motivation (Szulanski, 1996). An organization's social structure situates actors whom are within social reach of one another and thus are in a position to exchange their knowledge with specific others. This local and global social structure is to some degree dependent on the actors' task assignment (Van De Ven \& Delbecq, 1974; Van De Ven, Delbecq, \& Koenig, Jr., 1976). Moreover, actors' motivation to exchange their knowledge is clearly a factor as well. I investigate the extent to which these two high-level aspects of unifying organizations explain the level knowledge diffusion throughout the combined organization at a fixed point in time; this could also be reasoned as an investigation into the average speed of knowledge transfer in the organization.

Below, after elucidating and introducing the two overarching research questions (pertaining to structure, then motivation) - each corresponding to an independent virtual experiment--, I decompose each question into sub-constructs that are central to the question and also serve to operationalize it. Each sub-construct becomes a control variable in their 
respective virtual experiment. Below, I also elaborate on these constructs in detail and formulate specific research propositions which are used to guide the development of the overarching quantitative models and scientific response to the research questions.

\subsubsection{Structure}

Information flows from actor-to-actor according to the social network in which the actors co-exist, e.g., Phillips, Mannix, Nealec, and Gruenfeld (2004). Studies have shown that the structure of this network affects the dynamics of the flow of information throughout that social network. I factionalize the social network somewhat by paying attention only to the task-assignment network that the actor is a member of. I posit that the structure of this taskassignment network is a factor in the information flow and subsequence performance of the organization. The task-assignment structure in an organization is therefore an important feature of performance. For example, forming a large distributed team presents a moral hazard in the case of slacking or freeloading. I formalize a work breakdown and assignment structure as the task assignment structure for the organization. The task assignment structure is a function of the particular task that the business-line of the organization requires to be performed. There are two perspectives in structure (a) the actor perspective (actor specialization), and (b) that task perspective (team interdependence). The type of workers in important, e.g., independent knowledge workers (Kimberly \& Evanisko, 1981) as is the structure (Hinds \& Kielser, 1995).

Research Question 1: Structure: What are the quantifiable effects of the initial differences between Alpha and Beta on Task-Knowledge Transfer in NewCo, with respect to the antecedent, relative task-assignment structure? This is meant to explore how the manner in which work is assigned to and distributed among workers affects the knowledge transfer outcomes.

Underlying structure, I consider two sub-constructs: (a) the extent to which the actors specialize in their task assignments; this affects the shared-knowledge that is necessary among 
the work group, and (b) the degree to which the tasks are performed collaboratively and thus the amount of knowledge that is necessarily shared in the group.

\subsubsection{Structure: Actor Specialization}

Actor Specialization is regarded as the extent to which a worker is assigned, uniquely, to tasks, such that the worker is the only actor in a work team to be assigned to perform that task, i.e., the task-actor assignment from the task perspective is mutually exclusive. The more of these specialized tasks workers are assigned to, the greater the level of Actor Specialization in the organization. This could be thought of as one being an independent knowledge worker (see Osterloh \& Frey, 2000), e.g., a professional bowler.

In the work team, some individuals may specialize, which means they are assigned tasks, unique to others in the team. Actor Specialization can be realized as the number of tasks that an actor is assigned to uniquely, such that the actor is the only actor in a work team to be assigned to that task, i.e., the task-actor assignment from the task perspective is mutually exclusive. The greater number of these specialized tasks an actor is assigned to, the greater the Actor Specialization value. Actor Specialization refers to a non-interdependent knowledge worker (see Osterloh \& Frey, 2000, p. 546). These workers are not dependent on others when performing their tasks, they are independent knowledge workers, i.e., they do not necessarily require information from their team to perform, e.g., computer programmers, cashiers. The perspective of specialization is from that of the team. Specialization used herein, does not necessarily imply that an actor is limited to performing a single task, the actor may not have restricted task variety and indeed may be assigned to more than one task (see Narayanan, Balasubramanian, \& Swaminathan, 2009).

Proposition 1: Specialization: The organization performance is positively correlated with the level to which actors are task-specialists within their work-unit. 


\subsubsection{Structure: Team Interdependence}

I define team interdependence as the number of tasks that have multiple actors assigned to a single task and that the team members rely upon one another to mutually perform the work tasks (Van de Ven, Delbecq, \& Koenig, Jr., 1976; Jehn, 1995; Jehn, Northcraft, \& Neale, 1999).

This level of interaction and cooperation among individuals that must occur to perform a task, there is dependency and coordination necessary within a work team to perform a given task. For example, the level of team interdependence is central aspect of global virtual team communication patterns affecting performance (Maznevski \& Chudoba 2000). In one survey-based study, Janz, Colquitt, \& Noe (1997) found that reduced team interdependence affected job motivation and thus team effectiveness, though this relationship is highly mediated by the task being performed. Knowledge exchange is mediated by the present of weak / strong ties (Hansen, 1999); I presume, initially, that newly formed ties are weaker than the pre-existing ties during the post-merger integration stage.

I define team interdependence as the number of tasks that have multiple actors assigned to a single task and whom must complete tasks as a cooperative unit (e.g., Ellis, Hollenbeck, Ilgen, Porter, West, \& Moon, 2003; Hackman \& Morris, 1975). This level of interaction and cooperation among individuals that must occur to perform a task, there is dependency and coordination necessary within a work team to perform a given task. The more interdependence, the greater amount of transactive memory is necessary (Austin, 2003). For example, the level of team interdependence is central aspect of global virtual team communication patterns affecting performance (Maznevski \& Chudoba 2000). In academic definitions of team interdependence, it indicates how members rely upon one another to mutually perform work tasks (Van de Ven, Delbecq, \& Koenig, Jr., 1976; Jehn, 1995; Jehn, Northcraft, \& Neale, 1999).

The tasks assigned per actor variable for Beta is set relative to the Alpha setting and reflects the relative difference of TI between the two organizations. In the case of Alpha, each 
actor in a team will be assigned 3 of the 12 teams tasks. Beta's values will result in actors working on some tasks together as the tasks/actor value increases.

Proposition 2: Interdependence: The organization performance is positively correlated with the level to which task assignments require team interdependence within the work-unit.

Information-exchange motivation is comprised of two parts: (a) the willingness an actor has to seek out information, and (b) the willingness for an actor to share information with another. These two constituent parts form the basis for the overall absorptive capacity of the organization, which has been shown to affect the knowledge flow across units in multinational organizations (Gupta \& Govindarajan, 2000; Minbaeva, 2005; Minbaeva, Pedersen, Bjorkman, Fey, \& Park, 2003).

\subsubsection{Motivation}

Information flows are affected by the motivations of the individuals involved in the potential exchange. The extent to which an individual is motivated, or willing, to seek information from another person, clearly affects the flow of information to that individual. Moreover, the extent to which an individual is willing to share information with another when requested also clearly affects the exchange of information between the dyad, and therefore in the aggregate, throughout the organization. However, it is unclear the extent to which the organization-level is impacted given the heterogeneity of the members within the organization. There is a dilemma people face when considering their information exchange strategy and have several strategies that can be undertaken (see Larsson, Bengtsson, Henriksson \& Sparks, 1998), that directly affects the informational and brokerage power of the individual and those they chose to share or not to share their information with. Motivation has several dimensions, but is important to knowledge transfer (Osterloh \& Frey, 2000) and to the success of the organization as a whole (Osterloh, Frost, \& Frey, 2002). "Selfish individuals want to maintain their monopolistic position as holders of idiosyncratic 
knowledge" (Osterloh, Frost, \& Frey, 2002, p. 66), although sharing with your competition may be a self-positive strategy (Hamel, Doz, \& Prahalad, 1989).

Knowledge sharing by individuals is critical to organizational success (O’Dell\&Grayson, 1998; Osterloh \& Frey, 2000). Motivation is a factor in complete knowledge sharing both for the provider and recipient (Quigley, Tesluk, Locke, \& Bartol, 2007) and the communication attitudes along with actors having task knowledge has been shown to affect performance (de Vries, van den Hooff, \& de Ridder, 2006). There is extant literature on social identity issue in a merger setting (Boen, Vanbeselaere, \& Cool, 2006; Clark, Gioia, Ketchen, Jr., \& Thomas, 2010; Hogg \& Terry, 2000; Ullrich, Wieseke, \& Van Dick, 2005; van Knippenberg, van Knippenberg, Monden, \& de Lima, 2002; van Leeuwen, van Knippenberg, \& Ellemers, 2003). Recently, the notion of a transitional identity (Clark, Gioia, Ketchen, Jr., \& Thomas, 2010) has been suggested: during the integration period, individuals may take-on a temporary social identity as they transition from their pre-existing social identity into establishing a new social identity in the combined organization. For the exchange to be effective there needs to be shared understanding and mental models (Ko, Kirsch, \& King, 2005). Moreover, knowledge sharing is positively associated with performance (Du, Ai, \& Ren, 2007).

Research Question 2: Motivation: What are the quantifiable effects of the initial differences between Alpha and Beta on Task-Knowledge Transfer in NewCo, with respect to the antecedent, relative information-exchange motivation of organizational actors?

Information-exchange motivation is comprised of two parts: (a) the willingness an actor has to seek out information, and (b) the willingness for an actor to share information with another. These two constituent parts form the basis for the overall absorptive capacity of the organization, which has been shown to affect the knowledge flow across units in multinational organizations (Gupta \& Govindarajan, 2000; Minbaeva, 2005; Minbaeva, Pedersen, Bjorkman, Fey, \& Park, 2003). There is a circle of willingness to share and willingness to seek which spirals for either positively or negatively (Garud, Raghu, \& Kumaraswamy, 2005). 


\subsubsection{Motivation: Willingness to Seek}

Willingness to seek is the tendency for proactively reaching out for interaction opportunities that the actor has to interaction with another. As per Borgatti and Cross (2003) four factors, i.e., knowing, value, access, and cost, contribute to the determination of actual information seeking behavior by an individual when they evaluate reaching out to a prospective exchange partner. Furthermore, the underlying motivation to seek information and attempt to acquire from others is driven by organizational support (Eisenberger, Armeli, Rexwinkel, Lynch, \& Rhoades, 2001), social relationships (Borgatti \& Cross, 2003), among other numerous and antecedent factors involved in routine organizational activity (Crant, 2000; Wood \& Bandura, 1989) as well as in disruptive organizational change situations (Miller, Johnson, \& Grau, 1994). This willingness to seek behavior is also interrelated with help seeking behavior research, e.g., Nadler, Ellis, \& Bar, 2003; or, feedback-seeking behavior (Brett, Feldman, \& Weingart, 1990). For the exchange to be effective there needs to be shared understanding and mental models (Ko, Kirsch, \& King, 2005). Library Sciences research has found a bias towards seeking knowledge verbally and in-person over non-social information sources, such as databases, books, etc. (Weiler, 2005). Seeking information is an essential part of the group-newcomer socialization process (Morrison, 1993) and in the sense that the newcomer is trying to seek feedback reduce their environmental uncertainty (Ashford $\&$ Taylor, 1990) there seems there are parallels in the merger integration scenario. Moreover, the research into team learning behavior, e.g., Hirst, Van Knippenberg and Zhou (2009) indicate this is a complex phenomenon that supports performance, innovation, creativity and other essential individual, team and organizational processes.

Proposition 3: Willingness to Seek: The organization performance is positively correlated with the level to which individuals are willing to seek information from others.

\subsubsection{Motivation: Willingness to Share}

Willingness to share, an individual's inclination to share information, can be subject to numerous factors such as the type of information, organizational norms, or personal 
tendencies (Bock, Zmud, Kim, \& Lee, 2005; Constant, Kiesler, \& Sproull, 1994), which lead to a lack of motivation to share knowledge (Osterloh \& Frey, 2000), interpersonally or electronically virtual (Hsu, Ju, Yen, \& Chang, 2007; Kankanhalli, Tan, \& Wei, 2005; Raban, \& Rafaeli, 2007). Regardless that knowledge transfer results in benefit to an organization, numerous factors are in the influence sharing and transfer of information in an organization (Goh, 2002), which affect employee participation in this critical activity (Ardichvili, Page, \& Wentling, 2003). Information sharing is instrumental to team performance and while there are numerous moderators to the relationship, information sharing is positively and highly correlated with team performance (De Dreu, 2007; Mesmer-Magnus, \& DeChurch, 2009). Moreover, knowledge sharing is positively associated with overall organization performance (Du, Ai, \& Ren, 2007).

Proposition 4: Willingness to Share: The organization performance is positively correlated with the level to which individuals are willing to share information when interacting with others.

\subsection{Methodology}

\subsubsection{Simulated Merger Transaction}

In this simulation two autonomous, goal-seeking organizations are combined into one under the pretense of an organizational merger. Herein, I refer to the pre-merger organizations as Alpha and Beta, and the post-merger organization as NewCo. Strategically, Alpha and Beta merge in order to increase market share and thus expect to gain from economies of scale and various other synergies. Cost-cutting is not part of the strategy and workforce layoffs are of no concern, so the removal of actors from the organization is not a part of this simulated case; this is to say that all of the employees from both organizations will become members of NewCo.

This fictional, yet typical, case is construed to be a horizontal merger, so structurally both organizations have corresponding functional sub-units, e.g., accounting, sales, H.R., I.T., $\mathrm{R} \& \mathrm{D}$, etc. Both organizations are established businesses and are stable in all conceivable 
aspects; notably, prior to the merger, each is operationally performing at its maximum level. Each has reached a state of being a coherent and effective collection of integrated work-team comprised of highly-skilled (relative to their assigned tasks) and high-performing actors, all working with group-level purpose towards maximizing their individual-, work-team- and organization-level performance. Also stable and maximized, is the intra-team social network (Frantz, 2012) among the actors with inter-team ties establishing a cohesive organizational unit; any actor in the organization can plausibly interact with any other.

NewCo -- thus previously Alpha and Beta as well -- is formally organized with a work-team structure that, through the efforts of individuals within the work-team unit, aims to accomplish an explicit set of tasks. Each actor, whom is assigned to a specific work-team, performs their assigned tasks to the extent in which they have the necessary knowledge to perform that specific task. Such knowledge is acquired from others who are (or may be) sharing their knowledge with other actors, via typical social methodologies such as conversation, emails, etc. While the NewCo actors all now are members of the same social and organizational setting, because of their organization of origin (Alpha or Beta), task assignments and unique work histories, there is a probabilistic expectation of a difference in knowledge being held by each individual.

At the initialization of each virtual experiment, Alpha and Beta have just recently been merged into NewCo. The actors of each are commingled into teams through their task assignments under the amalgamated NewCo structure. Consequently, NewCo initially consists entirely of the pre-merger characteristics and pre-existing social networks of the actors from Alpha and Beta, conjoined only at the point of task-assignment. This situates $\mathrm{NewCo}$ as an eclectic mixture of socio-technical sub-networks that have simply become fused. Individual actors, in this virtual environment, now function, within the new task assignment structure, according to the social behavior theories implemented in the ascribed computational model; this computational model is described below. 


\subsubsection{Post-Merger Integration Setting}

The organizational merger transaction for this scenario is a single point-in-time event that involves combining the respective socio-technical networks of Alpha and Beta to form NewCo. At the initialization of the simulation, the functionally corresponding work teams from Alpha and Beta are matched and united with same-task work-team. Once the NewCo organization is setup in this manner, the behavioral model described above is in full control of the behaviors occurring within the virtual organization. This simulation does not differentiate between any behaviors between running in an ordinary organizational situation or in a postmerger situation.

\subsubsection{Computational Model}

These two experiments are conducted within the realm of a computational laboratory (Burton, 2003); in short, two computer simulations are similarly designed but conducted independently. The computational model of an organization used for these experiments is grounded in the Construct theory of social behavior as the theory is implemented in CONSTRUCT. CONSTRUCT is agent-based simulation software developed and maintained by CASOS (Carley, Martin, \& Hirshman, 2009). CONSTRUCT is based on the Carnegie School of social theory: people interact with one another within a network of social-technical relationships and exchange information with others in the network. CONSTRUCT has been used extensively in academic and governmental research. For extensive detail about CONSTRUCT and the underlying computational model and theories, see Appendix I in this article; additionally, readers are referred to any of the numerous descriptions by the authors (Carley \& Frantz, 2009; Frantz \& Carley, 2009a, 2009b).

\subsubsection{Representation Model}

The representational of an organization is based on the network paradigm and follows Dynamic Network Analysis (DNA; Carley, 2003) principles. This consistent with an agentbased simulation model (Elliott \& Kiel, 2004), and utilizes many of the features available in CONSTRUCT (Carley, 2002a, 2002b) software. In CONSTRUCT, relational networks are 
formed from combinations of three basic entity types: actors, knowledge and tasks. This model is an extension of PCANS (Krackhardt \& Carley, 1998), which represents an organization as a distinct set of stylized dyadic networks. From the three basic entity types, four classes of networks are formed: (a) a social network, which is an actor-to-actor relationship; (b) an assignment network consisting of actor-to-task relationships; (c) a task-toknowledge network which represents the concepts that are necessary to perform each task; and (d) a knowledge network which is an agent-to-task network representing what concepts each agent knows.

\subsubsection{Behavioral Model}

The behavioral model embedded in the individual agents for this simulation also relies upon the CONSTRUCT model (Carley, 1991). The Construct model has underpinnings based on social theories such as Symbolic Interactionism (Blumer, 1969), Structural Interactionism (Stryker, 1980), and Structural Differentiation Theory (Blau, 1970). The agent behaviors in the CONSTRUCT model are grounded in the information-processing perspective of the Carnegie School (Simon, 1957; March \& Simon, 1958; Cyert \& March, 1963), which models people as being information seeking agents. Moreover, CONTRUCT incorporates socialinformation-processing theory (Rice \& Aydin, 1991; Salancik \& Pfeffer, 1978), which posits that what knowledge a person has is largely dependent on the information they have access to via their social network. In CONSTRUCT, agents purposefully engage in multifaceted discourse (Dooley, Corman, McPhee, \& Kuhn, 2003) and social interaction (Carley, 1986) to exchange knowledge with one another. Agents regularly seek out others who have knowledge that they do not yet hold, and consequently are also being sought out by those seeking their knowledge. The selection of an interaction partner is complex and independently occurring, thus the formation of social groups is a self-organizing phenomenon (Guerin \& Kunkle, 2004). This complex interaction behavior is played out continually within an organization and mimics real-world behavior at an abstract level, leading to an approximation of the information diffusion process within an organization. 


\subsubsection{Operationalization of Constructs}

There are seven stylized constructs designed into this study: Four pertain to control variables (two constructs for each of the two experiments) and three to outcome variables (used in both experiments). These constructs are each described from an operational perspective below.

\subsubsection{Percentage of Task Knowledge (PTK)}

The percentage is calculated by the number of task-knowledge concepts that have ties in the actor-knowledge network (the number of actual) divided by the number of unique actor-knowledge ties required (task-knowledge times actor-task network times actorknowledge).

\subsubsection{Actor Specialization}

Actor Specialization is a value between 0 and 1 that indicates the proportion of the team tasks which are assigned to a specific actor, which are entirely unique to all other actors in the same team. That is, in the case of an actor being responsible for a specialized task, a given task has only actor assigned to accomplishing that task-represented by a Task node with only one tie and that tie being to a single actor node in the Task-Agent matrix. The Actor Specialization parameter is the percentage of tasks assigned to the actor that are uniquely tied to them, relative to the entire number of tasks assigned to the same actor, thus a value of 0.0 implies that the actor is assigned exclusively to tasks that other actors in the same team are assigned, e.g., a track-and-field athlete who participates in the 400 meter and 1,000 meter relay races. Whereas, a 1.0 implies that the actor is assigned to solo tasks exclusively; they have no co-workers on their assigned tasks, e.g. a track-and-field athlete who runs the 100meter and 400 meter sprint events. The Actor Specialization construct characterizes the specialist-generalist nature of the individuals in the given team. In some teams, individuals are specialists which suggest that they hold team knowledge that others in the team do not. Otherwise, some teams have a great deal of shared knowledge which is indicative of a team that has its membership consisting of less specialized members, thus having more generalist 
individuals. The notion of Actor Specialization has been studied in several academic studies, such as Ren, Carley, and Argote (2006), Austin (2003), and Child (1972).

\subsubsection{Team Interdependence}

Team Interdependence is a control parameter that indicates the fraction of agents $(n=12)$ within a single team that each previously unassigned task will be assigned to (assigned, after the actor specialization initialization process is completed). A value of 1.0 indicates that each (non-actor-specialized task) task is assigned to the full set of all actors in the work-team, implying a highly collaborative task, i.e., all members of the team work together on a given task. A value of 0.5 indicates that $1 / 2$ of the work-team is assigned to work on a given task, thus 6 actors are assigned. Using the track-and-field example, this value would indicate the fraction of team members that would be participating in a single relay race. Team Interdependence was abstracted closely in this way successfully by Lin (1994), and Lin and Carley (2003); Van Der Vegt, Emans, and Van De Vliert (1998, 2001) studied team interdependence empirically and informative research in the context of top management work teams has been published Barrick, Bradley, and Colbert (2007), and groups of others (Janz, Colquitt, \& Noe, 1997; Van Der Vegt, Van De Vliert. \& Oosterhof, 2003).

\subsubsection{Willingness to Seek}

Willingness to Seek is a proportion of the time-periods that an actor will be active in the interaction search process, i.e., the probability that a given actor will actively search for another actor to interact with in a given period. The parameter takes on a value between 0.0 and 1.0, representing the probability that the actor will reach-out to another to exchange information in each time cycle; the stochastic test is run independent for each time cycle. This parameter represents the information seeking tendency of individuals, socially. Having a willing to seek information from another, or perhaps seek advice, suggests a level of trust in the communications counterparty - or a level of information acquisition value assigned to the counterparty from the perspective of the focal actor; this phenomena is much studied, one that has been of interest to researchers in human communications research (Morrison, 2002; 
Morrison \& Vancouver, 2000), social networks (Borgatti \& Cross, 2003), and general management (Miller \& Jablin, 1991).

\subsubsection{Willingness to Share}

Willingness to share is the proportion of knowledge facts that an actor will consider to exchange with an interaction partner. This is a value between 0 and 1, with the lower bound value of 0 represents that the actor will consider sharing $0 \%$ of the knowledge the actor presently holds; to the upper bound at 1.0, the actor will consider all of their known knowledge facts when interacting with a partner. From this set of willing-to-share knowledge, a uniformly random selection is made to determine the specific concept to be shared with the exchange partner. Willingness to share is an approximation of individual trust and general cooperation between exchange partners. Information sharing has been studied for its effect on organization performance (Barrett \& Konsynski, 1982; Morishima, 1991). Moreover, willingness to share has been identified as a component of organizational culture (Connelly, \& Kelloway, 2003) and teamwork (Gigone \& Hastie, 1993), as it is specifically employed and designed in this study, and as an individual attitude within an organization (Bock \& Kim, 2002; Constant, Kiesler, \& Sproull, 1994).

\subsubsection{Experimental Design}

Two virtual experiments (computer simulations) are carried out to generate an extensive amount of detailed data; such a dataset is operationally impractical to acquire from traditional real-world cases or laboratory experiments. In each of the independently-executed experiments, four control variables are value-assigned and fixed in a factorial-designed fashion. Each variable is set to one of three possible and expressive values. Arising from this combination of variables and their respective initial values, there are 81 individual and unique experimental cells $(3 \times 3 \times 3 \times 3)$. Three outcome, or operationally dependent, variables will be computed at the end of each simulation time period. I execute 120 independent replications per experimental cell, which results in 9,270 independent and unique runs per each of the two experiments. 
The sole distinction in design of the two experiments is limited entirely to the set of four control variables which are experimentally manipulated and controlled at initialization; all other aspects of the simulations and experiments are identical. The various control and output constructs for the experiments are described and explained immediately below.

\subsubsection{Initialization}

The four control variables (two constructs for each of the two experiments) are initialized at the start of the execution of the simulation. In the case of the structure experiment, these values control the formation of the formal structure in the organization at time -1 and are not directly relevant during the execution of the simulation, as virtual time progresses. In the case of the motivation experiment, the control values directly affect the simulation each time period, though the values do not change whatsoever. The initial setting for the control variables are specified in Table 3-1. Recall, that this is a factorial experiment. Table 3-1. Initial settings of control variables.

\begin{tabular}{|c|c|}
\hline Control Variable & Set of Initial Values (fixed) \\
\hline \multicolumn{2}{|c|}{ Experiment: Structure } \\
\hline \multicolumn{2}{|c|}{ Actor Specialization } \\
\hline Alpha & $0.5,1.0,2.0$ \\
\hline Beta & $0.5,1.0,2.0$ \\
\hline \multicolumn{2}{|c|}{ Team Interdependence } \\
\hline Alpha & $0.1,0.3,0.4$ \\
\hline T Beta & $0.5,1.0 ., 2.0$ \\
\hline \multicolumn{2}{|c|}{ Experiment: Motivation } \\
\hline \multicolumn{2}{|c|}{ Willingness to Seek } \\
\hline Alpha & $0.2,0.4,0.5$ \\
\hline Beta & $0.5,1.0,2.0$ \\
\hline \multicolumn{2}{|c|}{ Willingness to Share } \\
\hline Alpha & $0.2,0.4,0.5$ \\
\hline Beta & $0.5,1.0,2.0$ \\
\hline
\end{tabular}

Note: 81 cells per experiment; 120 replications each cell; 999 time-periods each replication

\subsubsection{Termination}

The execution of each simulation is halted after 999 time periods. For analytic simplicity, only the final value of the relevant outcome measure, at time period 999 , is highlighted and analyzed in this study. I have pre-determined that this approach is sound. Previous trial runs of the experiment indicate that the outcome variables each are curvilinear 
asymptote over time; accordingly, their upper-bound value, unity (1.0), is a horizontal asymptote of these response curves. The shape of the over-time plot is a convex arc curve from 0.5 to 1.0. The curves are similar in shape and characteristic as the cumulative distribution function for an exponential distribution. At time period 999 some cases begin to reach unity (1.0) so continuing beyond this time period would be useless as the results would be indiscriminate thereafter. All outcome variables eventually reach unity (1.0) if the simulation is run endlessly; therefore, what differs is the asymptotic growth rates for each outcome variable. For simplicity, instead of approximating and analyzing coefficients of complex curves, I simply look where the outcome curve is at a fixed point in time (time period 999 suits this condition quite well), before all cases reach unity.

\subsection{Results}

In this section, I provide the quantitative results of the two virtual experiments: the first centered on structural factors and the second on motivational factors. Each experiment results in a large dataset that comprises a snapshot taken at virtual time period 999. These data are then applied in an ordinarily least squares regression process that results in estimates of the degree to which the controlled, pre-existing characteristics affect the amount of information diffused across the merged organization.

Table 3-2. Descriptive statistics for response variable, Percentage of Task Knowledge (PTK) at time period $999 ; n=9,720$ each experiment.

\begin{tabular}{llllll}
\hline & Mean & Median & Min. & Max. \\
\hline Experiment: Structure & 0.74 & 0.74 & 0.61 & 0.79 \\
& & & & 0.57 & 0.89 \\
\hline
\end{tabular}

First, in Table 3-2, I report basic descriptive statistics of the response variable for the experiments. Second, in Table 3 I provide the Ordinary Least Squares (OLS) regression results. Thirdly, via Equations 3-1 and 3-2, I show the quantitative regression models in 
complete form. Finally, I illustrate, graphically in Figure 3-1, the two models using their standardized coefficients. These are the quantitative results only; Qualitative interpretation, analysis and discussion are reserved for the Findings and Discussion Section, which follows this section.

Table 3-3. Regression results (Ordinary Least Squares).

\begin{tabular}{|c|c|c|}
\hline & $\begin{array}{c}\text { Intercept \& Coefficient } \\
(\beta) \\
\text { Estimates }\end{array}$ & $\begin{array}{c}\text { Standardized } \\
\text { Intercept \& Coefficient }(\beta) \\
\text { Estimates }\end{array}$ \\
\hline \multicolumn{3}{|l|}{ Experiment: Structure $\left(R^{2}=.96\right)$} \\
\hline Intercept & 0.731 & $0.738 * * *(0.000131)$ \\
\hline \multicolumn{3}{|l|}{ Specialization } \\
\hline Alpha & 0.124 & $0.155 * * *(0.000261)$ \\
\hline Beta Ratio & 0.100 & $0.122 * * *(0.000261)$ \\
\hline \multicolumn{3}{|l|}{ Team Interdependence } \\
\hline Alpha & 1.023 & $0.255 * * *(0.000261)$ \\
\hline Beta Ratio & 0.141 & $0.176 * * *(0.000261)$ \\
\hline \multicolumn{3}{|l|}{ Experiment: Motivation $\left(R^{2}=\right.$} \\
\hline \multicolumn{3}{|l|}{$96)$} \\
\hline Intercept & 0.450 & $0.723 * * *(0.000152)$ \\
\hline \multicolumn{3}{|l|}{ Willingness to Seek } \\
\hline Alpha & 0.506 & $0.126 * * *(0.000304)$ \\
\hline Beta Ratio & 0.075 & $0.093 * * *(0.000304)$ \\
\hline \multicolumn{3}{|l|}{ Willingness to Share } \\
\hline Alpha & 0.0002 & $0.0001 \quad(0.000304)$ \\
\hline Beta Ratio & 0.00003 & $0.00004(0.000304)$ \\
\hline
\end{tabular}

$*, * *, * * *$ indicate significance at the two-tailed $(0.10,0.05$, and 0.01$)$ levels, respectively. Standard errors are in parentheses.

Using the regression results shown in Table 3, I formulate two estimation models: Eqs. 3-1 and 3-2.

$$
P T K_{\text {estimated }}=73.1+12.4 b_{1}+10.0 b_{2}+102.3 b_{3}+14.1 b_{4}+\varepsilon
$$


Where $P T K_{\text {estimated }}$ is the structure model's estimate of the Percentage of Task Knowledge at time 999, constrained by $0 \leq P T K_{\text {estimated }} \leq 1 ; b_{1}$ is Actor Specialization for Alpha, with $b_{1}>0 ; b_{2}$ is Actor Specialization ratio for Beta, with $b_{2}>0 ; b_{3}$ is Team Interdependence for Alpha, with $b_{3}>0$, and; $b_{4}$ is Team Interdependence ratio for Beta, with $b_{4}>0$. The $\varepsilon$ variable is the residual error.

$$
P T K_{\text {estimated }}=45.0+50.6 b_{1}+7.5 b_{2}+0.02 b_{3}+0.003 b_{4}+\varepsilon
$$

Where $P T K_{\text {estimated }}$ is the motivation model's estimate of the Percentage of Task Knowledge at time 999, constrained by $0 \leq P T K_{\text {estimated }} \leq 1 ; b_{1}$ is Willingness to Seek for Alpha, with $0<b_{1} \leq 1 ; b_{2}$ is Willingness to Seek ratio for Beta, with $b_{2}>0 ; b_{3}$ is Willingness to Share for Alpha, with $0<b_{3} \leq 1$, and; $b_{4}$ is Willingness to Share ratio for Beta, with $b_{4}>0$. The $\varepsilon$ variable is the residual error.

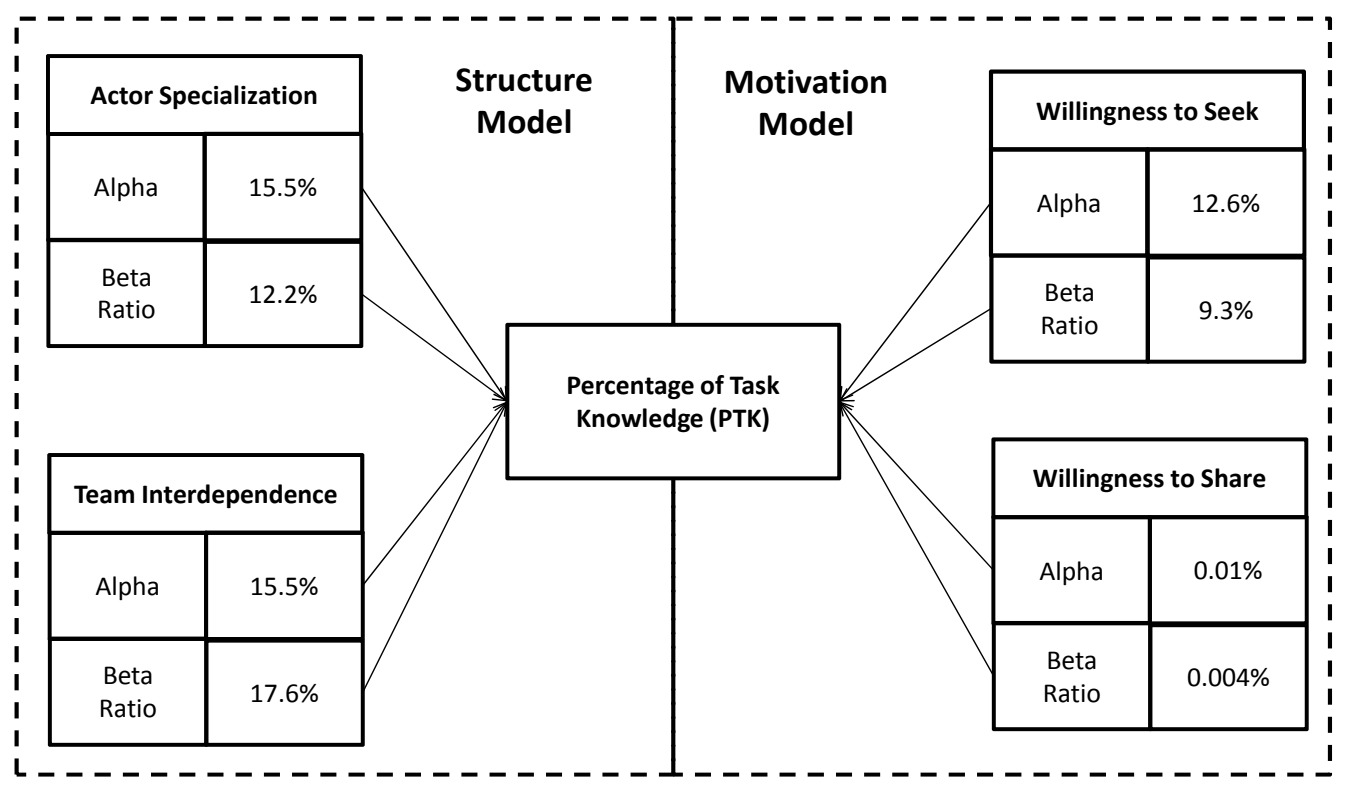

Figure 3-1. Construct Relationship Model (Standardized coefficients as a percentage).

\subsection{Discussion}

To explore the effects of task structure and motivation to exchange information on organization performance, I conducted two controlled, virtual experiments using agent-based, CONSTRUCT theory and software. With regard to structure, I focused on two features: (a) the level of task-specialization of the individuals, and (b) the team-level interdependence of 
individuals necessary to perform their tasks. Regarding motivation, I focused on two essential facets of information exchange: (a) individuals' willingness to seek information, and (b) individuals' willingness to share information. I consider the extent to which knowledge necessary for an individual (or group) to perform a task is a proxy for organizational performance; this is to recognize that a given tasked is accomplished to the extent that the individuals performing the task hold the knowledge specifically necessary to perform that particular task. I utilize a metric, Percentage of Task Knowledge (PTK), to serve as an organization-level aggregate measure of this concept of organizational performance.

Each virtual experiment consisted of 81 experimental cells, with 120 replications in each. Simulations were each stopped at time period 999 and the PTK measure logged at that point. The quantitative results of these experiments were detailed previously in the Qualitative Results Section, which includes a presentation of the ordinary least squares regression models that represent the data sets. In this section I qualitatively present the findings and discuss them phenomenologically.

\subsubsection{Structural Effects}

I find that the structure regression model met the broad expectations I initially put forth in the Introduction Section: The arithmetic sign of the regression coefficient in both instances is positive, as expected; thus, I confirm that actor specialization, team interdependence are both positively correlated with organization performance (Eq. 3-1). The coefficient of determination $\left(R^{2}=0.96\right)$ is near unity for the model; however, such high level of fit is not unexpected as this is ordinary in the case of controlled virtual experimentation.

Attending to the structure-based regression model (Eq. 3-1), the Specialization factor has coefficients of 12.4 and 10.0 for Alpha and Beta, respectively. Statistically, this difference between the two value is small and can be the attributed to sampling chance. I interpret the likeness of these two values as confirming the previously expected impact of the Beta variable; recall that Beta is a setting that indicates the Actor Specialization for the Beta portion of NewCo, relative to the Alpha setting, e.g., setting the beta value to 2.0 implies that 
for the Beta, the Actor Specialization setting is twice that of the Alpha setting for that instance and that 1.0 means that Alpha and Beta are the same setting. Actor Specialization contributes to performance in a manner that has a narrow target. Specific information needs to get to specific individuals to get specific tasks accomplished.

Turning our attention to Team Interdependence, the metric coefficient for the Alpha variable is worthy of extra emphasis due to its magnitude. I interpret that the coefficient of 102.3 for Alpha indicates the far-reaching relevance of team interdependence on the postmerger integration process. This metric coefficient suggests that high team Interdependence makes it much more likely that information is available to perform tasks because interdependences provides a wider net for specific information to get to an individual within a group. The conspicuously less value for the Beta coefficient can be interpreted as indicating that there may be a threshold that when surpassed provides less leverage to information diffusion in the combined organization. With Alpha being so high, the Beta Ratio has little relevance to the outcome.

\subsubsection{Motivational Effects}

I find that the motivation regression model met the broad expectations I initially put forth in the Introduction Section: The arithmetic sign of the regression coefficient in both instances is positive, as expected; thus, I confirm that willingness to seek and willingness to share are both positively correlated with organization performance (Eq. 3-2). The coefficient of determination $\left(R^{2}=0.96\right)$ is near unity for the model; however, such high level of fit is not unexpected as this is ordinary in the case of controlled virtual experimentation.

Attending to the motivation-based model (Eq. 3-2), the willingness to seek metric coefficient is 50.6 for Alpha and 7.5 for Beta. I surmise that the Beta is much smaller than that of Alpha for the same reason I concluded, above, that explained the Team interdependence condition. A threshold may have been reached so that increasing the willingness to seek for the Beta side of NewCo has less value if Alpha has a high value in willingness to seek. 
However, I are surprised about the near-zero value of the metric coefficient for the willingness to share variable. After investigating this apparent anomaly, I conclude that the willingness to share of any specific individual may actually be immaterial to the information exchange and thus organization performance because should a single actor not be responsive with information when approached for information, the numerous other actors in the organization can easily make up for that unresponsiveness when approached. This presumes that the unresponsive actor does not hold any particular knowledge exclusively to the extent that a large number of tasks require that exclusive knowledge. Therefore, it does make sense that willingness to share may not be a significant determinate of information flow in an organization. In the abstract, this situation is indicating a network affect that reflects the same phenomena as the Internet. The Internet is a computer network designed and structures to minimize the impact of, or resolve, specific node outages. If a given computer node goes out of service, the Internet is robust enough that the request for information, if it is redundant somewhere in the network, can still be satisfied even if one node is offline. This parallels what I found in the experiment. If a given actor refuses to share their knowledge, as long as that piece of knowledge is exclusively held by that to that one actor, the actor seeking that information will ultimately obtain it. I surmise, however, that this irrelevance hold true up to a certain threshold; there may be a level in an organization when a large enough number of actors, all who are unresponsive with no willingness to share, would result in a noticeable change in individual and organization performance. Determine that level would require more experimentation directed specifically toward that question is necessary.

\subsubsection{Conclusion}

Extending our analysis above, I put forth three stimulating conclusions that warrant further practitioner and academic investigation, empirically and experimentally. First, I abandon the common assumption that individuals' willingness to share information, hence their motivation, is entirely critical to knowledge diffusion and thus integration success; integration managers may safely not be overly concerned with motivating the merged organization to extensively share information with members of the acquiring organization. 
Second, I submit that an organization consisting of interdependent work-teams may require less attention than one consisting of independent work groups, while still achieving desirable organization performance. Thirdly, when it comes to information diffusion in a post-merger organization, it may be just as effective to focus on information exchange only to a point of critical mass rather than attempting to attend to all members of the combined organization; the outcome may be just as positive without all-encompassing effort. These three ideas somewhat conflict with what may be conventional (and unproven) management practice and orientation.

After subsequent pondering about the findings of these two experiments, I contemplate trying to grasp the broad difference of structural versus motivational factors in the post-merger integration scenario. Noticing the undeniable strength of the constituent factors in the structure experiment and the surprising weakness of those factors in the motivational experiment, I brazenly conjecture broadly that structural factors may actually be more critical than motivational factor during the post-merger integration process. This is congruent with research from academics that focus on organizational structure (e.g., Burton \& Obel, 1984; Sydow \& Windeler, 1998; Uzzi, 1997) and contrasts with motivational-focus researchers (e.g., Bunderson \& Sutcliffe, 2002; Jehn \& Shah, 1997; Lin, 2007; Martin-Cruz, Martin-Perez, \& Trevilla-Cantero, 2009; Martin-Perez, Martin-Cruz, \& Estrada-Vaquero, 2012; Miranda \& Saunders, 2003). Certainly more experimentation is necessary to better understand the relative effect difference between structure and motivation, along with empirical case-based investigation.

I conjecture that the methodological-based strength of this study suggests that these models have robust and theoretically-predictive power to quantitatively forecast dynamics in real-world mergers; however, this must be systematically confirmed through extensive laboratory and case-based study. This study accelerates a desire for additional development of detailed and reliable estimators and thus advances the obtainability of reliable forecasts which would be invaluable in real-world merger circumstances. 


\subsubsection{Limitations}

This study is based on a computational model and a computer simulation, which by design is a separation from the real-world. This paradigm relies upon abstraction but it is purposeful in the design of a model, e.g., the transfer of knowledge is modeled within a closed system. While the application of computational modeling is effective for developing organization theory with practical application (Dal Forno \& Merlone, 2004; Phelan, 2004), findings derived from artificial modeling should be applied with appropriate cautiousness (Bankes \& Lempert, 2004). Validation is imperative, but verification of the accuracy of these models can be a insurmountable (Burton, 1998; Keller-McNulty, Bellman, Carley, et al., 2006; Prietula \& Carley, 1994; Robinson, 1997) and comprehensive validation is difficult in the setting of the social sciences.

A notable limitation of this simulation is found in the partner-selection step of the exchange process: herein, only a dyad's knowledge-similarity determines the priority of potential exchange partners. This characteristic guides the likelihood that one actor will interact with another, thus affecting the speed in which little-known information is diffused throughout the broader organization. This phenomenon is a feature designed into the CONSTRUCT behavioral model in order to implement social homophily theory (Byrne, 1971). Future models might instead utilize other theoretical characteristics to select exchange partners, such as relative expertise, leadership style, and access to resources.

\subsubsection{Executive Summary}

This study advances our understanding of post-merger integration phenomenon and serves as an illustrative example of effectively applying agent-based modeling to management and organizational theory research. Virtual experiments were conducted to study the knowledge-transfer dynamics within a newly formed organization following the consummation of an organizational combination, i.e., merger, acquisition or alliance. Specifically, I focused on the effect of pre-existing - characteristics of the original 
organizations -- structural and motivational features on the knowledge transfer in the combined organization.

Detailed structural facets of pre-merger actor task-specialization and within-team interdependence, and motivational characteristics, such as information seeking and sharing are manipulated in a controlled manner to reflect the various profiles within a range of merger scenarios. The knowledge transfer rate of the new organization was examined with respect to the antecedent, experimental-control variables of each scenario. The experiments show that these factors do indeed vary in the amount of influence each has on the ensuing knowledgetransfer rate. Moreover, the combined strength of these factors has strong predictive power to quantitatively forecast the task-knowledge diffusion; these findings contribute to developing high-level, organization-performance projections and forecasts. I believe that this study has immediate relevance to future post-merger integration research and practice, by providing a theoretically sound, quantitative model that can be useful for rationally estimating the range of possible knowledge transfer outcomes in analogous organizational scenarios.

From the analysis of the experiment results, I put forward three ideas for integration managers: (a) managers may safely not be overly concerned about motivating the member of the acquired organization to share information with members of the acquiring organization, (b) an organization with lots of interdependent work-teams may require less attention during the post-merger integration than an organization made up of unconnected work groups, and (c) it may be just as effective to focus on information exchange only to a point of critical mass rather than attempting to attend to all members of the combined organization; the outcome may be just as positive.

I surmise that the strength of this study suggests that these models have robust and theoretically-predictive power to quantitatively forecast dynamics in real-world mergers; this must be confirmed through extensive laboratory and case-based study. This research facilitates additional development of detailed and reliable estimators and thus advances the reliable forecasts that may be invaluable in real-world merger situations. 


\section{CHAPTER 4: STUDY THREE}

\section{The Effects of Legacy Organization Culture on Post-Merger Integration}

\subsection{Abstract}

I explore the relationship between the characteristics of pre-existing organization cultures and post-merger integration dynamics; this study involves examining data produced by computer simulation. Two characteristics of organization culture, its characteristic complexity and its propensity for members' to share information, are controlled in computational experiments. To characterize post-merger integration dynamics, I measure the transfer of information with respect to two types: (a) that which is necessary in performing work tasks, and (b) that which underlies the features of a group's culture. The extent to which this information is common in a group is indicative of task performance and the cultural cohesiveness of its members; leading to the level of performance for the group. I consider cultural knowledge as it pertains to both that of the entire organization and at the work-team level; often times, these can be dissimilar. I find that cultural-complexity and exchangemotivation vary in their influence on the diffusion of task- and cultural-knowledge: the more complex the culture, the longer for post-merger integration to complete, while simultaneously task-performance suffers. However, the inclination for an organization to energetically share their culture with another group does not immensely impact the diffusion of cultural or task knowledge; moreover, high levels of task focus in a culture can hinder cultural diffusion, though performance is positively correlated with this characteristic. This study has relevance to post-merger integration research and practice by providing a theoretically-grounded, quantitative model useful for estimating the post-merger dynamics of cultural awareness and knowledge diffusion for a specific merger situation.

\subsection{Introduction}

An organization can be regarded as a repository for a body of knowledge (e.g., cultural tendencies, operational procedures, etc.) that is unique to any other organization. This body of knowledge is an important influence on the performance of the organization (Baum \& 
Ingram, 1998; Darr et al., 1995; Epple, Argote, \& Murphy, 1996) and can result in a competitive advantage for the organization (Argote \& Ingram, 2000; DeLong \& Fahey, 2000). The process of diffusing this knowledge throughout various actors in the organization varies widely (Argote, 1999). In the case of an organizational merger, success often requires that the pre-existing knowledge held by both organizations must be diffused widely throughout the new organization (Heo \& Yoo, 2002; Peltoniemi, 2007) to capture and maximize post-merger performance. Therefore, this study focuses on this diffusion process by looking at the existing, pre-established cultural characteristics of the two merging organizations to reveal their association with future post-merger outcomes. Our principal aim is to develop mathematical models, whose inputs are basic cultural features of the predecessor organizations, which can estimate post-merger organizational outcomes.

A central feature of an organization is its unique culture, which can be the root cause of failure (Vaughn, 1996), "indigestion” (Schein, 1990, p. 117), or success. Furthermore, an organization-level culture can have within it differing sub-cultures at the work-group level (Van Maanen \& Barley, 1985), consisting of both similar and dissimilar characteristics. The culture of the merging organizations affect the post-merger integration outcome; for example, an organization's formal structure has been shown to affect these dynamics (Frantz \& Carley, 2010), although the full impact of the informal structure remains an open area for investigation (Frantz, 2012).

In this study, which is part of a larger post-merger research program being conducted by the authors, I examine four aspects of organization culture: the complexity of the culture at the organization level, the complexity of the team-level culture, the groups' exchangemotivation and the task-focus orientation of its members. Cultural complexity can be regarded as the richness or exoticness of a culture and conceived as the number of attributes unique to a given culture; the greater the number of these unique attributes, the greater its complexity. This notion holds for culture complexity at both the organizational and the team levels. Every culture has some perceived level of complexity associated with it (Lundberg, 1985; Murdock \& Provost, 1973). Exchange-motivation is regarded as the members' enthusiasm for sharing information with others. Cultural exchange-motivation is the tendency for an individual to 
reach out to another to share information about their culture as well as their interest in accepting knowledge about a different culture from another. One's motivation to share and receive cultural knowledge is a characteristic of positive organizational citizenship (Jo \& Joo, 2011), which can be markedly influenced by the social structure of the organization (Lin, 1999; von Krogh, 2003). The tendency for sharing knowledge makes unique information accessible to others (Makino \& Inkpen, 2003); this information transfer enables cultural integration with and task performance to others in a group in which one identifies themselves (Nahapiet \& Ghoshal, 1999). Task-focus orientation can be regarded as the tendency of an individual to focus their attention on, and therefore increasingly communicate about, tactical and formalized information over and above more casual non-task oriented information, such as when engaging in social gossip. In this study, I question the extent to which cultural complexity, at the organizational and team levels, cultural outreach, and task focus are factors in the organizational dynamics and performance during post-merger integration.

\subsubsection{First Perspectives}

A potent feature of computational modeling and virtual experimentation using the agent-based paradigm is that the researcher has design control over behaviors of the virtual actors and the environment in which they behave. To provide the reader with important perspectives embodied in the virtual world crafted for this experiment, I elaborate on three central design perspectives: (a) I frame an organization as a collection of information processing agents that seek out, collect and exchange information among other actors, (b) I take the standpoint that a culture is fundamentally shared knowledge among the members of a group, and (c) I presume that task performance is directly correlated with holding the knowledge necessary to perform that task. These three perspectives are explained in more detail below.

\subsubsection{Organization as Information Processor}

I take the information-processing view of an organization: an organization is an organized collection of agents, each of which is an information processor. The Carnegie 
School of Organizational Theory (Cyert \& March, 1963; March \& Simon, 1958; Simon, 1957) proposed this information-processing perspective as a basis for organizational decision making. According to this information-centric perspective, individual behavior and ultimately organizational performance can be explained in terms of what information is available to an organization and specifically who within the organization holds the information; this critical for performing tasks effectively. In an organization, actors interact as a matter of routine, and in the course of their interaction, they exchange such information. This is to say that they exchange knowledge amongst one another as they interact, either through direct person-toperson communications or through indirect forms such as through electronic and broadcast media (Dooley \& Corman, 2004). The transfer of this knowledge throughout members of an organization is critical from social and organizational perspectives, and underpins concepts such as organizational learning (Argote, Gruenfeld, \& Naquin, 2000).

\subsubsection{Culture as Knowledge}

Culture is learned shared knowledge in a group (Reyes-Garcia et al., 2003) and is preserved through a socialization process (Schein, 1990) that involves transferring cultural information, through behavioral cues and artifacts, etc. In this study, I take anthropological and cognitive views of culture (Duranti, 1997), which regards culture as the sum total of a group's knowledge (Linton, 1940) consisting of the facts of the cultural norms, traditions, expectations, customary behaviors, etc. A contemporary example of such a norm in the organization setting is when and in what circumstances it is acceptable to use email over using direct verbal communication (El-Shinnawy \& Markus, 1998; Rowe \& Struck, 1999), or even submit to using social media. This knowledge of one's culture is held by each member of a group or organization in the form of numerous, specific knowledge concepts (D'Andrade, 1995; Kroeber \& Kluckholm, 1952; Romney, 1999). The members embedded in a culture intimately know detailed aspects about their culture which are implicitly and explicitly shared and reinforced with other in-group persons. Often such knowledge is held closely within the group and can sometimes intentionally be withheld from out-group persons in order to exclude unwanted others from the group. I take the perspective that a specific culture itself is a pool of shared and distinctive cultural information that is uniquely held by members of a 
specific group (D’Andrade, 1981), and that such information is not necessarily known or embraced by others outside of that particular group (Cremer, 1993). I must note that in practice, it may not be in the strategic interest that the combined organization meld the two pre-existing cultures into one (Vestring, King, \& Rouse, 2003).

\subsubsection{Task-Performance as Knowledge}

Naylor and Dickson (1969) offer that a factor of team performance is the structure of the task itself, and that the complexity of the task is then based on the amount of information required to perform that task. I adopt this similar perspective that task-performance is directly associated with task-knowledge, which is also aligned with other research (Beirly \& Chakrabarti, 1996; DeCarolis \& Dees, 1999). It follows, all other aspects being equal that the more task-specific knowledge an actor has for performing a given task, the better the actor will be at performing that task, and greater the amount of knowledge necessary for performing a given task, the higher the degree of difficulty will be for performing the task. Tasks can differ in the level of their complexity (Wood, 1986) and I assign the number of knowledge concepts to a specific task according to the complexity of that task, thus suggesting that a more complex task takes longer to master. An illustrative example of this would be a neighborhood pizza shop: if a pizza maker has no knowledge of how to make pizza, it would clearly result in a disappointing meal, while a pizza-maker with expert knowledge of how to make pizza, you can reasonably be assured you will have a delicious pizza nearly every time. This task knowledge often can be transferred from person to person and from group to group (Darr et al., 1995). I pragmatically acknowledge the influence of localized motivation on taskperformance, but for this study I remove such task-performance motivation from consideration in order to focus on the wider, more global cultural dimension of inclination for information exchange.

\subsubsection{Research Questions}

This study seeks to uncover the extent to which the cultural aspects of two pre-merger organizations explain the amount of cultural and task knowledge that is then diffused among 
members of the combined organization. I measure the amount of information transfer with respect to two distinct types of knowledge: (a) that which is necessary in performing work tasks, and (b) that which pertains to the particulars of a group's culture. This study considers cultural knowledge as it pertains to both the culture of the organization as a whole and at the work-team level; often times, these can concurrently be dissimilar cultures. I investigate the influence of cultural complexity and exchange-motivations on the post-merger diffusion of this task- and cultural-knowledge.

Hereafter, I refer to the two pre-merger organizations as Alpha and Beta, and the postmerger organization is referred to as NewCo. Formally stated this study involves two overarching research questions that each correspond to an independently executed virtual experiment:

\subsubsection{Research Question: Complexity}

What is the effect of pre-established cultural complexity on the diffusion of cultureand task-knowledge in the combined organization?

\subsubsection{Research Question: Exchange-Motivation}

What is the effect of pre-established cultural exchange-motivation on the diffusion of culture- and task-knowledge in the combined organization?

\subsubsection{Propositions}

Supportive of the two overarching research questions pertaining to characteristics of culture: its complexity and its exchange motivation; I craft numerous propositions to be directly considered in this study. These propositions are concisely summarized in Table 4-1, with each being presented and described at more length following. 


\subsubsection{Cultural Complexity: Organizational-Level}

Organizational-level cultural complexity can be regarded as the richness or exoticness of a culture and conceived as the number of attributes unique to a given culture; the greater the number of these unique attributes, the greater its complexity. This notion holds for culture complexity at both the organizational and the team levels. Every culture has some perceived level of complexity associated with it (Lundberg, 1985; Murdock \& Provost, 1973). For example, the Japanese are branded as having many nuanced traditions in their behaviors, protocols, norms and social cues, while American culture might be considered as simpler and somewhat less nuanced and therefore less complex. Moreover, I can conceive a metric to quantify the complexity of a culture by counting the number of specific cultural traits that are specific to a given culture. Thus, the Japanese might have a value of 10, while Americans have a culture complexity value of 3 . Of course, this would be difficult and impractical in reality, so consistent with computational models I am working here within an abstract realm. Simply put, the greater the number of these underlying cultural traits, the more complex the culture is. If forced to craft specific values, an especially simple approach to expressing this metric could be taken: one could satisfy a metric value by using a weighed form of the Hofstede (1983) values.

Table 4-1. Summary of Propositions (Proposition id \& expected directional influence).

\begin{tabular}{|c|c|c|c|}
\hline \multirow[b]{3}{*}{$\begin{array}{l}\text { Pre-existing } \\
\text { characteristics }\end{array}$} & \multicolumn{3}{|c|}{ Post-merger Integration Outcomes } \\
\hline & \multirow[b]{2}{*}{ Task-knowledge } & \multicolumn{2}{|c|}{ Culture-awareness } \\
\hline & & Organization-level & Team-level \\
\hline \multicolumn{4}{|l|}{ Cultural Complexity } \\
\hline Organization-level & 1a. negative & 1b. negative & 1c. negative \\
\hline Team-level & 2a. negative & 2b. negative & 2c. negative \\
\hline \multicolumn{4}{|c|}{ Cultural Exchange-Motivation } \\
\hline Outreach & 3a. negative & 3b. positive & 3c. positive \\
\hline Task-focus & 4a. positive & 4b. negative & 4c. negative \\
\hline
\end{tabular}

Cultural complexity is of immediate relevance in the post-merger integration process. In order to unify two cultures into a single culture in NewCo, members of Alpha will be 
exposed to the specific cultural traits of Beta, and visa-versa-possibly spawning an entirely new culture, for NewCo itself. The relative difference (Hofstede, 1983) between the two premerger cultures, in particular the difference in the complexity, will lead to a more complex integration. This is to say that the greater the number of these concepts that must be diffused, the greater the amount of human interaction necessary for transferring these concepts from one individual to another. This may have implications for task performance because instead of an interaction exchanging task-knowledge, organizational culture concepts are transferred in its place. Therefore, I propose the greater the number of these organizational culture concepts, the longer the time for complete culture-awareness to be reached at both the organization and team levels.

\subsection{Proposition 1a}

Organizational-level cultural complexity negatively affects the diffusion rate of taskknowledge.

\subsection{Proposition $1 b$}

Organizational-level cultural complexity negatively affects the diffusion rate of organizational-level culture-awareness.

\subsection{Proposition 1c}

Organizational-level cultural complexity negatively affects the diffusion rate of teamlevel culture-awareness.

\subsubsection{Cultural Complexity: Team-Level}

Team-level cultural complexity, similarly to organization-level culture complexity, can be regarded as a count of the specific characteristics that are unique to the culture of a specific work-team, relative to another such work-team in the organization; the greater the number of these concepts the more complex the culture is deemed to be (see the discussion above). Team-level cultural complexity has relevance in the post-merger integration process as within 
a specific NewCo team, the Alpha members will become exposed to the cultural concepts of Beta and vise-versa. The greater the number of these concepts that must be exchange and diffused throughout the team, the greater the number of dyadic interactions necessary for completing the total transfer of all these concepts from one individual to another. This may have implications for task performance because in a culture of high interaction exchanging task-knowledge, team culture concepts are transferred instead; thus, the greater the number of these team culture concepts, the longer the time for full dissemination to be reached.

\subsection{Proposition $2 a$}

Team-level cultural complexity negatively affects the diffusion rate of task-knowledge.

\subsection{Proposition $2 b$}

Team-level cultural complexity negatively affects the diffusion rate of organizationlevel culture awareness.

\subsection{Proposition 2c}

Team-level cultural complexity negatively affects the diffusion rate of team-level culture awareness.

\subsubsection{Cultural Exchange-Motivation: Outreach}

Exchange-motivation Outreach is regarded as the members' enthusiasm for sharing information with others. Cultural exchange-motivation is the tendency for an individual to reach out to another to share information they have with others. The tendency for sharing knowledge makes unique information accessible to others (Makino \& Inkpen, 2003); this information transfer enables cultural integration with and task performance to others in a group in which one identifies him/herself (Nahapiet \& Ghoshal, 1999). Some groups are quite proud of their culture and are self-energized to pass it on to others; they share who they are either directly, through stories, or through tangible artifacts such as posters on a wall. A high level of cultural outreach would suggest that individuals seek out others who differ from 
themselves and have exchanges that would have snippets such as "this is how we do it", or "we always do..." A low cultural outreach culture would result in overall less cultural transmission from the in-group to the unlike others, the out-group. For example, in an organizational culture that structures employee pay according to individual performance and is thus highly competitive, there could be less of this "free" advice being passed from one person to another, relative to a team-oriented culture supported by team bonus pay.

\subsection{Proposition $3 a$}

Culture-outreach negatively affects the diffusion rate of task-knowledge.

\subsection{Proposition $3 b$}

Culture-outreach positively affects the diffusion rate of organization-level cultureawareness.

\subsection{Proposition 3c}

Culture-outreach positively affects the diffusion rate of team-level culture-awareness.

\subsubsection{Cultural Exchange-Motivation: Task-Focus}

Task-focus orientation in a culture can be regarded as the tendency for individuals to focus their attention on, and therefore increasingly communicate about, tactical and formalized information. This would be opposed to a more relationship-oriented culture that communicates more casual non-task oriented information, such as when engaging in social gossip. One culture can be more task-oriented than another and then would be inclined to have more interactions involving task-knowledge exchange than other non-task knowledge. An example of a task-oriented culture might be an organization engaged in engineering design, or computer programming. A less task-focused culture might reside in an entrepreneurial organization. American culture is considered highly task-focused, while Japanese culture is considered less task-focused (Mujtaba \& Alsua, 2011). In academic 
research, it has been shown that a task-focus is more conducive to organization performance (Bass, 1967), though I take this view with caution as such findings are highly contextual.

\subsection{Proposition $4 a$}

Task-focus positively affects the diffusion rate of task-knowledge.

\subsection{Proposition $4 b$}

Task-focus negatively affects the diffusion rate of organization-level cultureawareness.

\subsection{Proposition $4 c$}

Task-focus negatively affects the diffusion rate of team-level culture-awareness.

\subsection{Methodology}

\subsubsection{Simulated Case Scenario}

In a typical merger scenario, two self-governing organizations, referred as Alpha and Beta, formally merge into a new self-governed organization, referred as NewCo. Strategically, Alpha and Beta are both seeking to gain a larger share of its market and when combined, NewCo hopes to gain economies of scale through various resource synergies. Cost-cutting is not part of NewCo's forward strategy; moreover, workforce redundancies are not of concern and therefore layoffs are not part of the simulated case scenario.

This is a horizontal merger; therefore, there are conceivably many structural and cultural similarities between Alpha and Beta. Both organizations are demographically and socially stable and each has matured into a coherent and industrious group of individuals working with a group-level purpose towards maximizing their organization's performance. There exists a tight intra-team social network (Frantz, 2012) among the actors with inter-team ties forming a united organization; anyone can interact with anyone else. Both organizations are organized by work units that, through the efforts of individuals within the unit, aim to 
accomplish a specific set of tasks. The individual actors, assigned to specific work-teams, perform their assigned tasks by accumulating knowledge that is obtained from others who are diffusing their knowledge in their interaction with other actors. However, though the actors navigate in the same social environment, because of their unique histories there is the prospect of cultural differences between the cultures of Alpha and Beta. These pre-merger cultural characteristics formulate the explicitly designed scenarios that will be implemented as the virtual experiments' control variables, which are later described in detail and substantiated in depth.

At the start of the virtual experiment, when Alpha and Beta are initially merged into NewCo, the actors of each are commingled at the team-level through task assignments under the NewCo structure. Consequently NewCo consists entirely of the pre-merger characteristics and pre-existing social networks of Alpha and Beta, conjoined at the point of task-assignment. This situates NewCo as a possibly eclectic mixture of socio-technical sub-networks that have become intermingled and must now interact according to the proscribed social behavior theories contained in the computational model, which I describe next.

\subsubsection{The Computational Model}

This research is conducted in a computational laboratory (Burton, 2003), specifically a computer simulation is designed and run. The organizational computational model used for these experiments is grounded in the Construct theory of social behavior as implemented in CONSTRUCT, which is software developed and maintained by CASOS (Carley, Martin, \& Hirshman, 2009). CONSTRUCT is grounded in the Carnegie School of social theory: people interact with one another within a complex network of social-technical relationships and exchange personally-held information, or knowledge between themselves. For deeper detail about the underlying computational model and simulation refer to various presentations by the authors (Carley \& Frantz, 2009; Frantz \& Carley, 2009a, 2009b). 


\subsubsection{The Representation Model}

The representational model of the organization for this agent-based simulation model (Elliott \& Kiel, 2004) is based on the CONSTRUCT model (Carley, 2002a, 2002b), which implements Dynamic Network Analysis (Carley, 2003) principles. Relational networks are formed from combinations of three basic entity types: actors, knowledge and tasks. This conceptual model is an extension of the PCANS model (Krackhardt \& Carley, 1998), which represents a functional virtual organization from a set of stylized, but simple and basic network forms. From the three entity types, four types of networks are formed. The first is the social network, which is an actor-to-actor relationship. The second is an assignment network consisting of actor-to-task relationships. The third is a task-to-knowledge network which represents the concepts that are necessary to perform each task. The fourth is the knowledge network which is an agent-to-task network representing what concepts each agent knows.

\subsubsection{The Behavioral Model}

The behavioral model of the agents for this simulation is also an operationalization of the CONSTRUCT model (Carley, 1991). Carley's Construct Theory has underpinnings from social theories such as Symbolic Interactionism (Blumer, 1969), Structural Interactionism (Stryker, 1980), and Structural Differentiation Theory (Blau, 1970). The behavioral aspect of the CONSTRUCT model takes the information-processing perspective of the Carnegie School (Simon, 1957; March \& Simon, 1958; Cyert \& March, 1963), which concludes that people are information seeking agents. CONTRUCT also integrates social-information-processing theory (Rice \& Aydin, 1991; Salancik \& Pfeffer, 1978), which states that what knowledge a person has is dependent on what information they have access to via their social network. In CONSTRUCT, agents purposefully interact through complex discourse (Dooley, Corman, McPhee, \& Kuhn, 2003) and social interaction (Carley, 1986) to exchange their knowledge with one another. Agents seek out others who have knowledge that they do not yet hold, and are sought out by others seeking their knowledge. The selection of their interaction partner(s) is dynamic and thus the formation of social groups is a self-organizing phenomenon (Guerin 
\& Kunkle, 2004). This interaction dynamic is played out numerous times within an organization and simulates real-world behavior at a conceptual level, leading to a reasonable approximation of information diffusion within an organization.

\subsubsection{The Merger Transaction Scenario}

The organizational merger transaction for this simulation is a single point-in-time event that involves combining the various socio-technical networks of Alpha and Beta to form NewCo. The respective work teams from Alpha and Beta are paired and combined with sametask work-team at the start of the simulation run. Once the NewCo organization is setup in this manner, the behavioral model described above is in full authority of the virtual behaviors occurring within the simulation from then on. This simulation does not differentiate any behaviors between running in an ordinary organizational situation or in a post-merger situation.

\subsubsection{Experimental Design}

Two virtual experiments (computer simulations) were carried out to generate extensive amounts of detailed data, which is operationally impossible to obtain from the traditional real-world cases or laboratory experiments. In each of the two experiments, the four control variables are set in a factorial-designed manner. They each have their value determined from a set of three possible values. From this combination of variables and initial values there are 81 individual experimental cells $(3 \times 3 \times 3 \times 3)$. Three outcome variables are computed at the end of each simulation time period. I execute 120 independent replications per cell, which results in 9,270 runs per experiment.

Each execution of the simulation is halted after 999 time periods. For analytic simplicity only the final value of the outcome measure, at time period 999, is presented and analyzed in this report. Trial runs of the experiment indicate that the outcome variables each are curvilinear asymptote as time advances. Accordingly, their upper-bound value, unity, is a horizontal asymptote of these response curves. The shape of the outcome is convex arc curve 
from 0.5 to 1.0 . It is at time period 999 that some samples begin to hit unity so looking beyond this time period the results would be indiscriminate. All outcome variables reach unity if the simulation are run forever, what differs therefor is the asymptotic growth rates for each outcome variable. For simplicity, instead of dealing with complex curves, I simply look where the outcome curve is at a fixed point in time (time period 999 suits this condition quite well), before all reach unity.

The only difference between the two experiments is the set of four control variables and their values used to initialize the simulation; all other aspects of the two simulations are identical. The constructs for both experiments explained in the following section.

\subsubsection{Constructs and Initialization}

There are four constructs making up the control variables (two constructs for each of the two experiments) and three outcome constructs (used in both experiments). These constructs are each described in Table 4-2. The control constructs each have two initialization values, one for pre-existing Alpha organization and one for the pre-existing Beta organization. The Alpha value is an absolute value, while the Beta value is a value which is relative to the Alpha value. For example, for Culture Outreach Alpha may be assigned a setting of 0.4, and Beta's Culture Outreach setting may be 2.0, which indicates that Beta's culture outreach setting is set to $0.8(0.4 \times 2.0)$. This means that Beta's Culture Outreach is twice that of Alpha's Culture Outreach setting. For the Task Focus variable, however, Beta will have an absolute value, in the same style as for Alpha. The initial values of the control variables are presented in Table 4-3.

Table 4-2. Glossary of Constructs.

\begin{tabular}{|c|c|}
\hline \multicolumn{2}{|c|}{ Construct Name } \\
\hline Definition & Operational Definition \\
\hline Cultural Complexity Organizational-Level \\
\hline
\end{tabular}


The complexity of a culture refers to the number of unique traits held by a culture (Denton, 2004; Hannerz, 1991; Murdock \& Provost, 1973; Sackmann, 1997). In an anthropological study of 100 societies, hundreds of such unique traits were uncovered (Carneiro, 1970); this is one example study that actually quantifies the notion of culture according to the number of concepts identified and making up the unique aspects of various cultures. Murdock and Provost (1973) developed a process for quantifying a culture, as has Chick (1997), while their different procedures are not suitable for use in this computational model, they have developed methods to quantify different cultures that can indicate differences in their complexity.
The count of unique and abstract culture concepts pertinent to the entire organization; value is a whole number zero or greater. A number of unique culture concepts are assigned to each Alpha and Beta team prior to the merger. After the merger, these concepts are exchanged during the dyadic interactions. This construct has a corresponding setting for Beta, but is designed as a relative ratio value-relative to the assigned value for Alpha.

\section{Cultural Complexity Team-level}

The same meaning as described above, but in the context of individual workteams. Groups, or work-teams, within a culture often have a subculture that differs from that of the broader organization (Hannerz, 1991; Sanchez, Lanero, Yurrebaso, \& Tejero, 2007).
This is operationalized in the same manner as for the organization-level construct, but instead this indicates the number of concepts per work-team within the organization.

\section{Culture Outreach}


The prospect that an actor will seek out interaction with others who are different than themselves according to the knowledge both hold at the time. According to homophily theory, people tend to interact with similar others. If an actor has a tendency to seek out different others they increasingly serve as a social bridge between homophily groups and become a conduct for the exchange of information that is not already known to the group (Benito \& Gripsrud, 1992). In the setting or an organizational merger, individuals proud of their culture and work performance is more motivated to share information with those perceived as being less fortunate, according to the focal individual; this is a form of cultural pride (Pullar, 1992) and expansion (DiMaggio \& Useem, 1978). Empirically, in the case of the 1990s merger of Morgan Stanley and Dean Witter, there was a tendency for the Morgan Stanley group to reach out to the Dean Witter group during causal interactions and promote the merits of the firms culture and work ethic.
The mean value for the knowledge similarly weight network which indicates the probably of interaction across specific dyads. Cultural outreach is a value in the range $0-1$, which is passed to CONSTRUCT as a parameter to the knowledge similarity weight network. The parameter is passed as the mean value for the weight network generation that occurs at the initial stage of the simulation. The greater this value, the more likely the individual will seek out others that are less like them relative to their knowledge base. This construct has a corresponding setting for Beta, but is designed as a relative ratio value-relative to the assigned value for Alpha.

\section{Task Focus}

Task focus is the tendency for an actor to provide information in an exchange with another actor that pertains to their
The priority assigned to the knowledge priority network for the actor/knowledge dyad, which influences the 


\begin{tabular}{|c|c|}
\hline $\begin{array}{l}\text { assigned tasks, versus providing cultural } \\
\text { information in that exchange. Several studies } \\
\text { have uncovered the relevance of task focus in } \\
\text { group relations. Brewer and Miller (1988), in } \\
\text { a study on racial desegregation, found } \\
\text { positive effects when opposed groups focus } \\
\text { on task in their interaction. Studies by Smith, } \\
\text { Carroll, and Ashford (1995) and Yilmaz, and } \\
\text { Hunt (2001) both found that groups can } \\
\text { productively cooperate if they are focused on } \\
\text { accomplishing a particular task. Moreover, } \\
\text { Bettencourt, Brewer, Croak, \& Miller (1992) } \\
\text { found that such task-focused cooperation } \\
\text { tended to reduce intra-group bias. }\end{array}$ & $\begin{array}{l}\text { selection of the specific knowledge concept } \\
\text { transferred in an interaction between two } \\
\text { actors. This is a categorical value \{Low, } \\
\text { Medium, High\} which is translated then } \\
\text { passed to CONSTRUCT as a parameter to the } \\
\text { knowledge priority weight network. The } \\
\text { parameter is passed as a setting for each agent } \\
\text { to knowledge pair that sets a weighting to the } \\
\text { knowledge for each individual that affects the } \\
\text { selection of which knowledge concept (task } \\
\text { of cultural) is exchanged in a given dyad. The } \\
\text { higher this setting, the more likely the task } \\
\text { concepts will be favored over the cultural } \\
\text { concepts when a dyad exchanges information } \\
\text { during an interaction. This construct has a } \\
\text { corresponding setting for Beta, which is } \\
\text { independent of the Alpha setting. }\end{array}$ \\
\hline Percentage of Task Knowledge & \\
\hline $\begin{array}{l}\text { Task knowledge refers to the set of } \\
\text { specific information that is required to } \\
\text { completing a specific task. In this study, these } \\
\text { information concepts are those that are } \\
\text { unique to performing a specific task in a } \\
\text { specific organization or work-group. For } \\
\text { example, in a bookkeeping task, knowledge } \\
\text { of how to manually record a cash sale is } \\
\text { common knowledge across two organizations } \\
\text { and is therefore not captured in this virtual }\end{array}$ & $\begin{array}{l}\text { A knowledge entity that has "task" as } \\
\text { an attribute. This entity will have } \\
\text { membership in the task-knowledge and the } \\
\text { actor-knowledge networks. The percentage is } \\
\text { computed by the number of task-knowledge } \\
\text { concepts that have ties in the actor- } \\
\text { knowledge network (the number of actual) } \\
\text { divided by the unique number of actor- } \\
\text { knowledge ties required (task-knowledge } \\
\text { times actor-task network times actor- }\end{array}$ \\
\hline
\end{tabular}




\begin{tabular}{|c|c|}
\hline $\begin{array}{l}\text { environment. However, knowledge of how to } \\
\text { run the automated bookkeeping system in one } \\
\text { organization differs from the other and is } \\
\text { therefore considered as a task knowledge } \\
\text { concept in this virtual environment. }\end{array}$ & knowledge). \\
\hline \multicolumn{2}{|c|}{ Percentage of Team-Level Culture (PTC) } \\
\hline $\begin{array}{l}\text { Knowledge concepts that pertain to } \\
\text { the unique characteristics of a specific-team's } \\
\text { culture, consisting of knowledge concepts. } \\
\text { These are unique knowledge entities that } \\
\text { make up the actor-knowledge network } \\
\text { specific to actor-team membership. }\end{array}$ & $\begin{array}{l}\text { A knowledge entity that has "team- } \\
\text { culture" as an attribute. This entity will have } \\
\text { membership in the task-knowledge and the } \\
\text { actor-knowledge networks. The percentage is } \\
\text { computed by the number of these team- } \\
\text { culture concepts in the task-knowledge } \\
\text { network that have ties in the actor-knowledge } \\
\text { network (the number of actual) divided by the } \\
\text { unique number of actor-knowledge ties } \\
\text { required. }\end{array}$ \\
\hline \multicolumn{2}{|c|}{ Percentage of Organization-level Culture (POC) } \\
\hline $\begin{array}{l}\text { Knowledge concepts that pertain to } \\
\text { the unique characteristics of the organizations } \\
\text { culture, consisting of knowledge concepts. } \\
\text { These are unique knowledge entities that } \\
\text { make up the actor-knowledge network } \\
\text { specific the actor's original organizational } \\
\text { membership, either Alpha or Beta. }\end{array}$ & $\begin{array}{l}\text { A knowledge entity that has } \\
\text { "organization-culture" as an attribute. This } \\
\text { entity will have membership in the task- } \\
\text { knowledge and the actor-knowledge } \\
\text { networks. The percentage is computed by the } \\
\text { number of these organization-culture } \\
\text { concepts that have ties in the actor- } \\
\text { knowledge network (the number of actual) } \\
\text { divided by the number of Alpha and Beta } \\
\text { knowledge entity concepts combined. }\end{array}$ \\
\hline
\end{tabular}


Table 4-3. Parameter Values of Control Variables.

\begin{tabular}{|c|c|}
\hline Control Variable & Assigned Values \\
\hline \multicolumn{2}{|c|}{ Experiment: Complexity } \\
\hline \multicolumn{2}{|c|}{ Organizational-Level Cultural Complexity } \\
\hline Concepts per Organization Alpha & $10,30,50$ \\
\hline Concepts per Organization Ratio Beta & $0.5,1.0,2.0$ \\
\hline \multicolumn{2}{|l|}{ Team-Level Cultural Complexity } \\
\hline Concepts per Team Alpha & $2,6,18$ \\
\hline Concepts per Team Ratio Beta & $0.5,10 ., 2.0$ \\
\hline \multicolumn{2}{|c|}{ Experiment: Exchange-Motivation } \\
\hline \multicolumn{2}{|l|}{ Culture Outreach } \\
\hline Outreach Alpha & $0.2,0.4,0.5$ \\
\hline Outreach Ratio Beta & $0.5,1.0,2.0$ \\
\hline \multicolumn{2}{|l|}{ Task Focus } \\
\hline Task Focus Alpha & Low, Medium, High \\
\hline Task Focus Beta & Low, Medium, High \\
\hline
\end{tabular}

\subsection{Results}

I conducted two virtual experiments: one exploring complexity characteristics and the second exploring the exchange-motivation characteristics of the cultures of the pre-merger organizations. Both experiments, separately, establish the degree to which pre-existing, cultural characteristics affect the diffusion level at time period 999 of: (a) task-knowledge, (b) organizational-level culture awareness, and (c) team-level culture-awareness. In this section, I report the quantitative outcomes from conducting these experiments.

Table 4-4 reports descriptive statistics of the response variables for the two experiments and Ordinary least squares (OLS) regression results for the experiments are presented in Table 4-5. 
Table 4-4. Descriptive statistics for response variables, $n=9,720$ each experiment.

\begin{tabular}{lccccc}
\hline & & Min. & Mean & Median & Max. \\
\hline Experiment: Complexity & & & & & \\
& PTK & 0.61 & 0.73 & 0.73 & 0.86 \\
PTC & 0.64 & 0.76 & 0.76 & 0.91 \\
POC & 0.86 & 0.93 & 0.94 & 0.98 \\
Experiment: Exchange-Motivation & & & & \\
PTK & 0.53 & 0.70 & 0.70 & 0.78 \\
PTC & 0.65 & 0.72 & 0.71 & 0.82 \\
POC & 0.51 & 0.87 & 0.95 & 0.97 \\
\hline
\end{tabular}

Table 4-5. OLS Regression Results.

\begin{tabular}{|c|c|c|}
\hline & $\begin{array}{c}\text { Coefficient } \\
\text { Estimate (B) }\end{array}$ & $\begin{array}{c}\text { Standard } \\
\text { Coefficient } \\
\text { Estimate (b) }\end{array}$ \\
\hline \multicolumn{3}{|c|}{ Experiment: Complexity PTK $\left(R^{2}=.91\right)$} \\
\hline Intercept & 0.863 & $0.726 * * *(0.000139)$ \\
\hline \multicolumn{3}{|c|}{ Organization Culture Complexity } \\
\hline Alpha & -0.002 & $-0.061 * * *(0.000278)$ \\
\hline Beta Ratio & -0.024 & $-0.029 * * *(0.000278)$ \\
\hline \multicolumn{3}{|c|}{ Team Culture Complexity } \\
\hline Alpha & -0.004 & $-0.056 * * *(0.000278)$ \\
\hline Beta Ratio & -0.015 & $-0.018 * * *(0.000278)$ \\
\hline \multicolumn{3}{|c|}{ Experiment: Complexity PTC $\left(R^{2}=.86\right)$} \\
\hline Intercept & 0.901 & $0.760 * * *(0.000190)$ \\
\hline \multicolumn{3}{|c|}{ Organization Culture Complexity } \\
\hline Alpha & -0.002 & $-0.062 * * *(0.000379)$ \\
\hline Beta Ratio & -0.024 & $-0.030 * * *(0.000379)$ \\
\hline \multicolumn{3}{|c|}{ Team Culture Complexity } \\
\hline Alpha & -0.004 & $-0.058 * * *(0.000379)$ \\
\hline Beta Ratio & -0.016 & $-0.020 * * *(0.000379)$ \\
\hline \multicolumn{3}{|c|}{ Experiment: Complexity $\mathrm{POC}\left(R^{2}=.87\right)$} \\
\hline Intercept & 0.998 & $0.934 * * *(0.000083)$ \\
\hline \multicolumn{3}{|c|}{ Organization Culture Complexity } \\
\hline Alpha & -0.001 & $-0.026 * * *(0.000166)$ \\
\hline Beta Ratio & -0.013 & $-0.016 * * *(0.000166)$ \\
\hline
\end{tabular}




\begin{tabular}{|c|c|c|}
\hline Alpha & -0.002 & $-0.027^{* * *}(0.000166)$ \\
\hline Beta Ratio & -0.008 & $-0.009 * * *(0.000166)$ \\
\hline \multicolumn{3}{|c|}{ Experiment: Exchange-Motivation PTK $\left(R^{2}=.51\right)$} \\
\hline Intercept & 0.656 & $0.644 * * *(0.000609)$ \\
\hline \multicolumn{3}{|l|}{ Culture Outreach } \\
\hline Alpha & -0.020 & $-0.005^{* * *}(0.000545)$ \\
\hline Beta Ratio & -0.005 & $-0.006 * * *(0.000545)$ \\
\hline \multicolumn{3}{|l|}{ Task Focus } \\
\hline Alpha Medium & 0.033 & $0.033 * * *(0.000667)$ \\
\hline Alpha High & 0.024 & $0.024 * * *(0.000667)$ \\
\hline Beta Medium & 0.047 & $0.047 * * *(0.000667)$ \\
\hline Beta High & 0.051 & $0.051^{* * *}(0.000667)$ \\
\hline \multicolumn{3}{|c|}{ Experiment: Exchange-Motivation PTC $\left(R^{2}=.64\right)$} \\
\hline Intercept & 0.701 & $0.690 * * *(0.000519)$ \\
\hline \multicolumn{3}{|l|}{ Culture Outreach } \\
\hline Culture Outreach alpha & -0.017 & $-0.004 * * *(0.000464)$ \\
\hline Culture Outreach ratio Beta & -0.004 & $-0.005^{* * *}(0.000464)$ \\
\hline \multicolumn{3}{|l|}{ Task Focus } \\
\hline Task Focus Alpha Medium & 0.015 & $0.015^{* * *}(0.000568)$ \\
\hline Task Focus Alpha High & -0.023 & $-0.023^{* * *}(0.000568)$ \\
\hline Task Focus Beta Medium & 0.053 & $0.053^{* * *}(0.000568)$ \\
\hline Task Focus Beta High & 0.056 & $0.056^{* * *}(0.000568)$ \\
\hline \multicolumn{3}{|c|}{ Experiment: Exchange-Motivation POC $\left(R^{2}=.75\right)$} \\
\hline Intercept & 0.657 & $0.644 * * *(0.001539)$ \\
\hline \multicolumn{3}{|l|}{ Culture Outreach } \\
\hline Culture Outreach alpha & -0.022 & $-0.005^{* * *}(0.001377)$ \\
\hline Culture Outreach ratio Beta & -0.004 & $-0.006 * * *(0.001377)$ \\
\hline \multicolumn{3}{|l|}{ Task Focus } \\
\hline Task Focus Alpha Medium & 0.172 & $0.172^{* * *}(0.001686)$ \\
\hline Task Focus Alpha High & 0.220 & $0.220 * * *(0.001686)$ \\
\hline Task Focus Beta Medium & 0.136 & $0.136 * * *(0.001686)$ \\
\hline Task Focus Beta High & 0.160 & $0.160 * * *(0.001686)$ \\
\hline
\end{tabular}

$*, * *, * * *$ indicate significance at the two-tailed $(0.10,0.05$, and 0.01$)$ levels, respectively. Standard errors are in parentheses.

Using the results reported in Table 4-5, I formulate six estimator models, Eqs. 4-1 through 4-6, which are presented below. 


$$
\begin{aligned}
& P T K=86.3-0.2 b_{1}-2.4 b_{2}-0.4 b_{3}-1.5 b_{4} \\
& \text { PTC }=90.1-0.2 b_{1}-2.4 b_{2}-0.4 b_{3}-1.6 b_{4} \\
& \text { POC }=99.8-0.1 b_{1}-1.3 b_{2}-0.2 b_{3}-0.8 b_{4}
\end{aligned}
$$

Whereas PTK is the Percentage of Task Knowledge at time 999, constrained by $0 \leq$ $P T K \leq 1 ; P T C$ is the Percentage of Task-level Culture at time 999, constrained by $0 \leq P T C \leq$ 1; $P O C$ is the Percentage of Organization-level Culture at time 999, constrained by $0 \leq P O C$ $\leq 1 ; b_{1}$ is Organization-level culture complexity for Alpha, with $b_{1}>0 ; b_{2}$ is Organizationlevel culture complexity ratio for Beta, with $b_{2}>0 ; b_{3}$ is Team-level culture complexity for Alpha, with $b_{3}>0$, and; $b_{4}$ is Team-level culture complexity ratio for Beta, with $b_{4}>0$.

$$
\begin{aligned}
& P T K=65.6-2.0 b_{1}-0.5 b_{2}+3.3 b_{3}+2.4 b_{4}+4.7 b_{5}+5.1 b_{6} \\
& P T C=70.1-1.7 b_{1}-0.4 b_{2}+1.5 b_{3}-2.3 b_{4}+5.3 b_{5}+5.6 b_{6} \\
& P O C=65.7-2.2 b_{1}-0.4 b_{2}+17.2 b_{3}+22.0 b_{4}+13.6 b_{5}+16.0 b_{6}
\end{aligned}
$$

Whereas PTK is the Percentage of Task Knowledge at time 999, constrained by $0 \leq$ $P T K \leq 1 ; P T C$ is the Percentage of Task-level Culture at time 999, constrained by $0 \leq P T C \leq$ $1 ; P O C$ is the Percentage of Organization-level Culture at time 999, constrained by $0 \leq P O C$ $\leq 1 ; b_{1}$ is Culture outreach for Alpha, with $0 \leq b_{1} \leq 1 ; b_{2}$ is Culture outreach ratio for Beta, with $b_{2}>0 ; b_{3}$ is the Task Focus Medium dummy variable for Alpha, with $b_{3} \in\{0,1\} ; b_{4}$ is the Task Focus High dummy variable for Alpha, with $b_{4} \in\{0,1\} ; b_{5}$ is the Task Focus Medium dummy variable for Beta, with $b_{5} \in\{0,1\}$, and; $b_{6}$ is the Task Focus High dummy variable for Beta, with $b_{6} \in\{0,1\}$. Figure 4-1 provides a graphical presentation of the six models (Eqs. 4-1 to 4-6). 


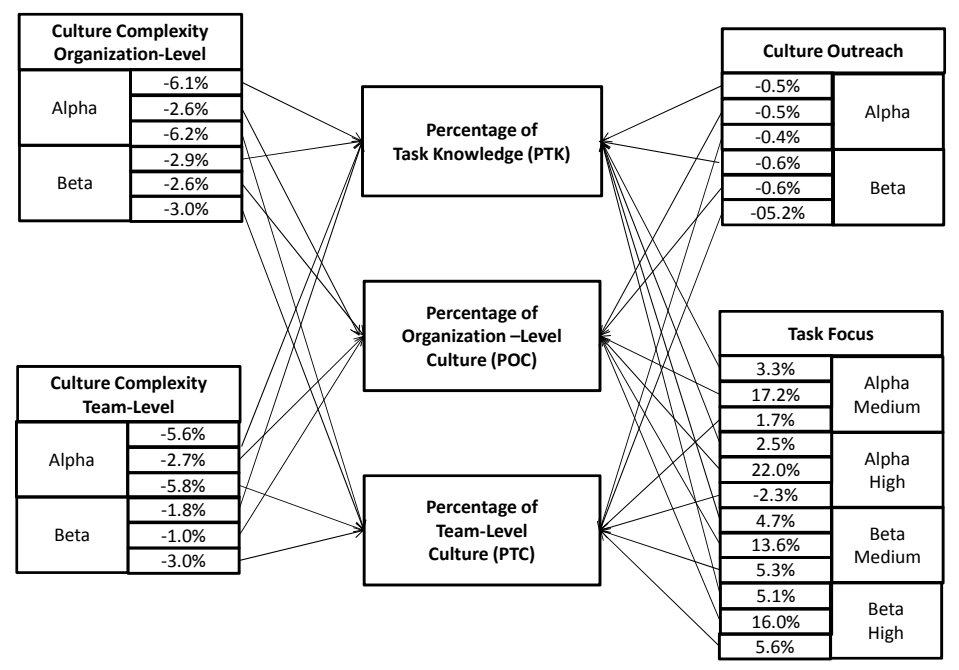

Figure 4-1. OLS-based Construct Relationship Model.

\subsection{Discussion}

To explore the relationship of pre-existing organization culture with integration dynamics following an organizational merger, I conducted two controlled virtual experiments, using the CONSTRUCT model. I focused on two key features of organization culture: (a) its characteristic complexity, and (b) the members' exchange motivation. To characterize postmerger integration dynamics, I measure the level of information transfer with respect to two distinct types of knowledge: (a) that which is necessary in performing specific work tasks, and (b) that which pertains to the particulars of a group's culture. Separately, these are indicators of the work performance of the organization and of the cultural cohesiveness within the organization. The virtual experiments involved cultural knowledge at both the organization and the team level, independent of one another.

\subsubsection{Cultural Complexity}

Looking first at the results of the cultural complexity experiment, the signs of the beta coefficients of the three models (Eqs. 4-1 to 4-3) constructed are consistent with the directional expectations summarized in Table 1. A high level of cultural complexity in the 
pre-merger culture clearly increases the length of the diffusion process with respect to the diffusion of cultural information at both the organizational and team levels. Moreover, cultural complexity also negatively affects the diffusion of task knowledge, as expected according to previous theory. A simple analysis of the y-intercepts for Eqs. 4-1 to 4-3 also shows clearly that due to the near-to-100 values the factors in the model, namely organization complexity at the organization and team level, can only hinder the speed of diffusion of cultural and task knowledge throughout the merged organization. These equations also indicate that complexity of organization-level culture has a greater influence in the overall culture-awareness and task-knowledge diffusion rates than does the complexity of the teamlevel culture, although these differences re negligible. The task-knowledge diffusion (Eq. 4-1 and Table 4-3), indicates that there is not a great deal of difference in the impact of organizational complexity relative to the complexity at the team-level. Moreover, looking at the standardized coefficients for the two culture metrics (POC \& PTC; Table 4-3), there is not a strikingly difference in the betas either. Although, at the fine level the complexity of organization-level culture has more of an influence in the overall culture-awareness and taskknowledge diffusion rates than does the complexity of the team-level culture. From this review of the cultural complexity experiment, I discern that culture complexity does indeed increase the length of time for diffusion of culture at the organization and team levels, and that culture complexity does also negatively affect the diffusion of task knowledge throughout the organization. I also find that there is not a marked difference in the impact of culture complexity at the organization versus the complexity at the team level.

\subsubsection{Exchange Motivation}

Looking at the exchange-motivation experiment, a comparison of theoretic expectations and the experiment results (Table 4-1 \& Eqs. 4-4 to 4-6) immediately presents us with an unexpected outcome: in the case of Percentage of Organization-level Culture (POC) and Percentage of Team-level Culture (PTC), shown by Eqs. 4-5 \& 4-6, culture-outreach has a negative effect on the diffusion of culture; rather than having the expected positive effect; moreover, task-focus has a positive effect, rather than a negative effect as theory expects. 
Both results are counter to what I expected from previous conjecture. I see that the motivational aspect of the pre-established cultures is positively correlated with the rate of diffusion, although the importance of its constituent parts is more difficult to quantify and assess with as much confidence. Note that a high level of task-focus is more a factor for the diffusion of organization- level culture-awareness, but a low level of task-focus is more relevant for the diffusion of team-level culture-awareness. I posit that this is due to the inherent nature of working as a team that task-knowledge will diffuse merely as a requirement for individuals to perform; individuals need to exchange task-knowledge regardless of the characteristic of their culture. I suggest that an explanation for this is that the organizational members have highly-prioritized the transmission of task information to the extent that cultural information is left withheld in the individual members and thus not diffused to others. An explanation for this unexpected result also may be that by the increasing focus on task knowledge creates a situation of increased knowledge-similarity between two actors. Individuals increase the probability that they will select one another as interaction partners. As the frequency of this interaction increases, the likelihood of an exchange occurring between the pair increases even more so; coupled with having already exchanged the prioritized task-related knowledge, the pair then exchanges the remaining culture-related concepts; leading to increased culture-awareness diffusion within individuals and thus the organization as a whole. To summarize, these unexpected results in the exchange-motivation experiment indicate that: (a) increased cultural outreach tendencies do not result in more rapid culture-knowledge diffusion, and (b) an increased level of task focus positively contributes to the increased diffusion of cultural knowledge, both for the organizational and team levels. In short, within a culture, motivation is indeed pertinent to diffusion, but the outcome is directional according to the type of motivation. A high motivation for sharing task knowledge is quite supportive of the post-merger integration process, while a high motivation for sharing cultural information is less supportive of the integration process. 


\subsubsection{Combined}

Combining the two experiments, I find that cultural-complexity and exchangemotivation vary in their influence on the diffusion of task- and cultural-knowledge. The more complex the culture the longer for post-merger organizational and team cultural integration to occur; task performance also suffers. However, the inclination for an organization to energetically share their culture with another group does not immensely impact the diffusion of cultural or task knowledge. Moreover, a task focus in a culture can benefit both culturaland task-knowledge diffusion.

From the above review, I can conclude by evaluating the quantitative results from the virtual experiments that the designed model used for generating the simulated data adequately corresponds with established organizational theory. Our broad expectations of how the model would behave according to our theory-based propositions (Table 4-1) were generally consistent with regression estimators that are derived from the outcome data (Table 4-5 and Eqs. 4-1 to 4-6), though I uncovered some surprise. Therefore, I am comfortable with forming some broad conclusions according to experiment results and the discussion presented in prior sections.

\subsubsection{Quantitative Models}

I have constructed a quantitative model (Eqs. 4-1 to 4-6) that can provide a functional response to the two research questions put forth at the start of this study: (a) what is the effect of pre-established cultural complexity on the diffusion of culture-and task-knowledge in the combined organization, and (b) what is the effect of pre-established cultural exchangemotivation on the diffusion of culture-and task-knowledge in the combined organization? The models derived from these experiments can be used for estimating the task-knowledge exchange and the culture exchange rates well before the consummation of a merger; these models can be a potent planning tool. By applying this model it is possible to offer an initial estimated baseline assessment of the dynamics of a real-world merger from the perspective of diffusion of culture-awareness and task-knowledge during an integration stage. There is 
obviously much more research to be done, but this early model is a step forward to the development of even richer quantitative models. Future work, using this same research methodology, includes examining other facets of culture to develop other quantitative models.

\subsubsection{Qualitative}

From a qualitative perspective, I can conclude that pre-existing cultural features, namely cultural complexity and exchange-motivation, vary in the influence each has on the diffusion of task and cultural knowledge. The more complex the culture, the greater the time for post-merger organizational and team cultural integration to complete, while simultaneously task-performance suffers as well. However, the inclination for an organization to energetically share their culture with another group does not immensely impact the speed of diffusion of cultural or task knowledge, which is counter-intuitive. Moreover, high levels of task focus in a culture can hinder culture-related diffusion, though performance is positively correlated with this characteristic. Overall, I suggest that two cultures both having a balance of culture-outreach and task-focus has the speediest post-merger integration time.

From these experiments, the intricate idea of motivation clearly requires much more research. I can quickly deduce that the organizational characteristics of culture-outreach and task-focus are not simple factors, and I suspect at a minimum there may be an important interaction between these two that need to be studied more closely and modeled with more

precision. Some of the work will be in the experimental design and some likely in the modeling aspect of these sort of virtual experiments.

I conjecture that the strength of this study suggests that these models have robust and theoretically-predictive power to quantitatively forecast dynamics in real-world mergers; this of course must be confirmed through case study. Ultimately this research facilitates further development of detailed and reliable estimators and thus advances the improvement of reliable forecasts which may be invaluable in real-world merger situations. 


\subsubsection{Limitations}

A virtual experiment, as with any methodology, inherently has limitations. While the application of computational models can be an effective method for developing organization theory with practical application (Dal Forno \& Merlone, 2004; Phelan, 2004), findings derived from such a methodology should be applied with caution (Bankes \& Lempert, 2004). Validation is important, but authentication of these models can be a challenge (Burton, 1998; Keller-McNulty, Bellman, Carley, et al., 2006; Prietula \& Carley, 1994; Robinson, 1997) and total validation could be considered impossible in the setting of the social sciences. Consequently, computational models are often relegated to provoking thought and discussion rather than being absolutely predictive.

The most noteworthy limitation of this study is found in the partner-selection step of the simulated exchange process, only a dyad's knowledge-similarity determines the priority of potential exchange partners. This dyad-based metric directs the likelihood that one actor will interact with another. This, therefore, affects the speed in which minority, or little known, information is diffused throughout the organization. This phenomenon is legitimacy designed into the CONSTRUCT behavioral model according to social homophily theory (Byrne, 1971). Future modelers could also consider other characteristics such as relative expertise, leadership style, and resource availability in this partner selection process. 



\section{CHAPTER 5: QUANTITATIVE RESULTS}

This chapter includes a restatement of the constituent studies' results as well as a synthesis of these results newly unified, which offers a quantitative perspective not accessible in the individual studies. This chapter also provides a short note on the overall validity of the computer model employed in these studies. However, before delving into these quantitative results, several clarifications must be elucidated so that one does not misinterpret these models; these important points are presented next.

\subsection{Clarifications}

Below, seven clarifying points are presented to establish the context within which the following models should be construed; these include both what is and what is not. Since this study is conducted in a virtual laboratory, the first points presented are notice to the reader about the areas of the experiments' departure from the real world - that is the computational model's high-degree of abstractness. Later points are notices about how to properly interpret unique aspects of the data and results. These points may be construed as limitations; indeed, any model representing reality is froth with reductions, but in the realm of the social sciences, these abstractions are the power of such models. Herein, these abstractions are purposeful design decisions that stimulate the generalization of the results. These points identify the cognitive boundaries separating the model and experiments from the real-world phenomena; these pin-point several departures from the mental model of reality and interpretations that reader will typically make.

\subsubsection{Abstract Entities}

The computational models employed herein are highly abstract representations of reality; understanding the extent of this abstraction is critical to properly conceptualizing the results. If the results are understood purely on their own without also understanding the magnitude of the abstractness, the reader may be misdirected. Consistent with the methodology, only a portion of the real-world is designed into the virtual experiments; what is 
included or not is entirely purposeful. The notion of a Task in the model is left precisely unspecified as a given task could be as wide-spread as performing the bookkeeping function for billion-dollar corporation, or as minute and mundane as the task of tying a shoelacethink shoe-shine person at the train station. Another example is the notion of Knowledge concept (or a distinct piece of information); recall, a Task requires specific Knowledge for the Task to be performed. A single Knowledge concept could be as majestic as knowing how to pilot an Airbus 380 superliner, or as minute as knowing the capital city of France.

The critical issue with this abstraction is that the units across these models remain the same, or at least are consistent; of course, these units themselves can be quite abstract. Again, the task of being a bookkeeper for the large company is in no way comparable to the task of tying a shoelace. These two tasks are a grave mismatch and are rather incompatible from many dimensions - for example, in the complexity of performing the task, if complexity is the unit—, therefor, they should not be modeled within the same simulation. A more comparable and equitable match of tasks could be the bookkeeping task and the task of managing the sales department, for example. These two are arguably similar in their complexity to perform, though still distinctly different tasks. To restate, the unit of measure itself does not necessarily matter, but what is paramount is the consistency of employing the same unit across the same entities in the entire model.

\subsubsection{Dissimilar Features Only}

These experiments do not replicate the post-merger integration process in its grand entirety; instead, only the concepts of knowledge, or information, that are unique to one of organizations are involved. Diffusion of knowledge that others already hold is not socially dynamic and thus does not warrant scientific investigation. However, the diffusion of knowledge that is held only by some and not others in an organization is indeed dynamic and thus scientifically motivating. Conversely, only those Tasks which are common to the two organizations are included in the model. It is this intersection of performing tasks that necessitate the need for knowledge transfer across merging organizations. With this in mind, the interpretation of the result must be strictly maintained within this context: two knowledge- 
identical organizations merging are not expected to offer dynamics; just as adding cold water to cold water, versus adding boiling water to cold. Therefore, with these two rubrics, the outcome variable, the organization performance metric, is not a holistic metric as typically interpreted; it should not be construed as the performance of the entire organization, per se. The performance measure is a percentage ratio that pertains strictly to those tasks included in the modeled and pertains to the portion of the task that relies upon the information unique to the organization. The relevance of this performance ratio depends on an estimate of its weighting relative to the task information that is commonly available in both organizations.

\subsubsection{Time Unit is Abstract}

Unit of time in these models is an abstract concept that may have little discernable direct association to real-world time. While exact transformation from virtual time to realtime is left to individual interpretation, the relativeness of one time period to another in the simulation is appropriate. In the simulation each time period represents one complete cycle of the communications process within the organization. The time unit, a cycle, is consistent throughout the simulation, though mapping it to real-world could be conceivable but might be rather complex and unreliable. For example, each time period could be mapped to one 8-hour business working day, though it would be a rare day at work if an actor exchanged only a single item of information to an individual, but this depends on the meaning, or magnitude, of the transferred knowledge concept. The clock time to share knowledge related to flying an airplane has a different magnitude than the clock time to share knowledge on tying a shoelace. Furthermore, in some organizations, the work days do not include weekends, and so forth.

\subsubsection{Non-Zero Outcomes}

At no point in the simulation does an actor have zero performance, and therefor at no point does the organization have zero performance. In fact, performance at time zero begins at $50 \%$ since, by design, the required task-information initially is spread amongst the two organizations evenly - they are designed to be in the same line of business. Also, not all experiments start at exactly the same performance level, though most do begin at $50 \%$. Any 
differences are dependent on the control variable settings but are strictly consistent within an experimental cell.

\subsubsection{Outcome Trajectory}

In these models the organization learning, thus knowledge diffusion, is always growing - knowledge does not get lost or depreciate; it only accumulates. Once an actor learns or holds a specific knowledge concept, that concept is never detached from that actor. Subsequently, the organization performance, over sequential time-steps, will always follow a monotonic increasing trajectory. The form of the over-time trajectory is consistent with a cumulative distribution curve for an exponential function; it monotonically increases from 0.5 to 1.0. The notion of forgetting is not implemented in this study, though it certainly is a phenomena reasonably present in the real-world. However, in an organizational merger, the loss of knowledge, either through forgetting, or though depreciation, is not a relevant dynamic as the length of real-world time for the integration is not usually suggestive of skill-loss or obsolesce. This organizational model does not implement the concept knowledge having a half-life (Winter, 1987). This trajectory suggests that on the outset, there is a great deal of new knowledge exchanged that over time such a rate diminishes as the amount of new knowledge left to exchange approaches zero. There is more fresh information to be exchanged and as time goes on, information exchanges between dyads is increasingly redundant information. The rate of change - the slope of the performance-level curve-approaches zero as more information becomes redundant and less is learned in each successive time period.

\subsubsection{Single Data-Point}

These data are captured and reported at a single point in virtual time only-time period 999. Recall that time is not mapped to the real-world and the trajectory between time 0 and 999 is not necessary a linear second derivative. This single data-point capture approach results in some lost fidelity reaching into the early stages of the integration period, but because of the similarity of the path across all experiments there is limited value - relative to the research question - in adding additional analytic complexity. 


\subsubsection{Comparative Analysis}

Because of the abstractness of the computational models, the results of the experiments have restricted use if applied to a particular merger scenario in isolation. Instead, the results are highly valued in a comparative analysis setting-comparing two different scenarios for the same merger, or comparing two different potential merger targets for a same acquirer. More extensive explanation of how to use the results in an applied setting-along with illustrative examples - is provided in Chapter 7 Conclusions.

\subsection{Models From Constituent Studies}

Each of the constituent studies presents their distinctive results in the respective published article (see Chapters 2, 3, and 4). Recall, the methods and the experiments across the three studies are the same; only the focal control and outcome variables differ. For convenience, the resulting quantitative models from the studies are repeated in this section.

5.2.1 Study One: "Post-Merger Knowledge Transfer: Examining Antecedent Structural Complexity":

Task-Knowledge level $=0.7217+0.0003 * b_{1}-0.0200 * b_{2}-0.0002 * b_{3}-0.0020^{*} b_{4^{-}}-0.0016^{*} b_{5^{-}}$ $0.0247 * b_{6}$

$$
\begin{aligned}
& \text { Where } \quad b_{1}=\text { time } \\
& b_{2}=\text { organization complexity } \\
& b_{3}=\text { agents per team alpha } \\
& b_{4}=\text { agent per team ratio aloha to beta } \\
& b_{5}=\text { knowledge concepts per team alpha } \\
& b_{6}=\text { knowledge concepts per team ratio alpha to beta }
\end{aligned}
$$

and Task-Knowledge Level is a continuous value between 0.0 and 1.0, inclusive. This model has an $R^{2}$ of $88 \%$, which is high.

5.2.2 Study Two: "Post-Merger Integration: Effects of Task Structure and Motivation to Exchange Information on Organization Performance"

$$
P T K_{\text {estimated }}=73.1+12.4 b_{1}+10.0 b_{2}+102.3 b_{3}+14.1 b_{4}+\varepsilon
$$


Where $P T K_{\text {estimated }}$ is the structure model's estimate of the Percentage of Task Knowledge at time 999, constrained by $0 \leq P T K_{\text {estimated }} \leq 1 ; b_{1}$ is Actor Specialization for Alpha, with $b_{1}>0 ; b_{2}$ is Actor Specialization ratio for Beta, with $b_{2}>0 ; b_{3}$ is Team Interdependence for Alpha, with $b_{3}>0$, and; $b_{4}$ is Team Interdependence ratio for Beta, with $b_{4}>0$. The $\varepsilon$ variable is the residual error.

$$
P T K_{\text {estimated }}=45.0+50.6 b_{1}+7.5 b_{2}+0.02 b_{3}+0.003 b_{4}+\varepsilon
$$

Where $P T K_{\text {estimated }}$ is the motivation model's estimate of the Percentage of Task Knowledge at time 999, constrained by $0 \leq P T K_{\text {estimated }} \leq 1 ; b_{1}$ is Willingness to Seek for Alpha, with $0<b_{1} \leq 1 ; b_{2}$ is Willingness to Seek ratio for Beta, with $b_{2}>0 ; b_{3}$ is Willingness to Share for Alpha, with $0<b_{3} \leq 1$, and; $b_{4}$ is Willingness to Share ratio for Beta, with $b_{4}>0$. The $\varepsilon$ variable is the residual error.

5.2.3 Study Three: "The Effects of Legacy Organization Culture on Post-Merger Integration":

$$
\begin{aligned}
& P T K=86.3-0.2 b_{1}-2.4 b_{2}-0.4 b_{3}-1.5 b_{4} \\
& \text { PTC }=90.1-0.2 b_{1}-2.4 b_{2}-0.4 b_{3}-1.6 b_{4} \\
& \text { POC }=99.8-0.1 b_{1}-1.3 b_{2}-0.2 b_{3}-0.8 b_{4}
\end{aligned}
$$

Whereas $P T K$ is the Percentage of Task Knowledge at time 999, constrained by $0 \leq$ $P T K \leq 1 ; P T C$ is the Percentage of Task-level Culture at time 999, constrained by $0 \leq P T C \leq$ 1; $P O C$ is the Percentage of Organization-level Culture at time 999, constrained by $0 \leq P O C$ $\leq 1 ; b_{1}$ is Organization-level culture complexity for Alpha, with $b_{1}>0 ; b_{2}$ is Organizationlevel culture complexity ratio for Beta, with $b_{2}>0 ; b_{3}$ is Team-level culture complexity for Alpha, with $b_{3}>0$, and; $b_{4}$ is Team-level culture complexity ratio for Beta, with $b_{4}>0$.

$$
\begin{aligned}
& P T K=65.6-2.0 b_{1}-0.5 b_{2}+3.3 b_{3}+2.4 b_{4}+4.7 b_{5}+5.1 b_{6} \\
& \text { PTC }=70.1-1.7 b_{1}-0.4 b_{2}+1.5 b_{3}-2.3 b_{4}+5.3 b_{5}+5.6 b_{6} \\
& \text { POC }=65.7-2.2 b_{1}-0.4 b_{2}+17.2 b_{3}+22.0 b_{4}+13.6 b_{5}+16.0 b_{6}
\end{aligned}
$$

Whereas PTK is the Percentage of Task Knowledge at time 999, constrained by $0 \leq$ $P T K \leq 1 ; P T C$ is the Percentage of Task-level Culture at time 999, constrained by $0 \leq P T C \leq$ 1; $P O C$ is the Percentage of Organization-level Culture at time 999, constrained by $0 \leq P O C$ $\leq 1 ; b_{1}$ is Culture outreach for Alpha, with $0 \leq b_{1} \leq 1 ; b_{2}$ is Culture outreach ratio for Beta, 
with $b_{2}>0 ; b_{3}$ is the Task Focus Medium dummy variable for Alpha, with $b_{3} \in\{0,1\} ; b_{4}$ is the Task Focus High dummy variable for Alpha, with $b_{4} \in\{0,1\} ; b_{5}$ is the Task Focus Medium dummy variable for Beta, with $b_{5} \in\{0,1\}$, and; $b_{6}$ is the Task Focus High dummy variable for Beta, with $b_{6} \in\{0,1\}$.

\subsection{RESULTS META-ANALYSIS}

Considering the results from the three experiments, in the aggregate, a meta-analysis provides valuable insight into the dynamics of a merger by looking at all of the factors sideby-side and combined. By combing the beta coefficients from all of the experiments' models into a single rank-ordering list offers insight that is not readily apparent in any single experiment. In addition, looking at the coefficients from their classification of being for a structural or motivational variable provides important insight. A meta-analysis of the three articles is provided below. First, the rank-ordering of control variables is presented, followed by an interpretation of the results.

\subsubsection{Interaction Effects}

The findings of Study Three (see Section 4.3.5, also Section 6.2), in particular, warrant an investigation into possible interactions between the independent variables in this study. To investigate this, additional analysis is necessary that go beyond the pre-planned experiments and analysis. Typically, deeper analysis is conducted on a combined dataset, in this case, data from all five experiments reported in this research. Phenomenologically, such a secondary investigation into a multiplicative effect between the structure of the culture and the characteristics of the culture can be carried out by simply rerunning an ordinary least squares multivariate regression model on the combined data. However, due to the nature of the data, being from controlled experiments - the independent variables are purposefully contrived for this factorial-designed experiment(s), rather than phenomenologically random-there exists extensive conditions of singularity, leading to multicollinearity problems with the interaction terms, leading to un-usable regression results from the combined dataset. 
For example, Figure 5-1 graphically illustrates the relationship between the two independent variables found in separate experiments (experiments \#1 and \#2), Actor Specialization Beta Ratio and Organization Culture Complexity; their correlation is exactly 1.0. The scatter-plot shows the correlation of the 68,040 data points, which is limited to just 4 unique datapoints on the dataplot. To work around this issue, separate and additional experiments must be conducted (See Sections 7.6 and 7.6).

Figure 5-1. Pearson correlation of two independent variables: Combined dataset.

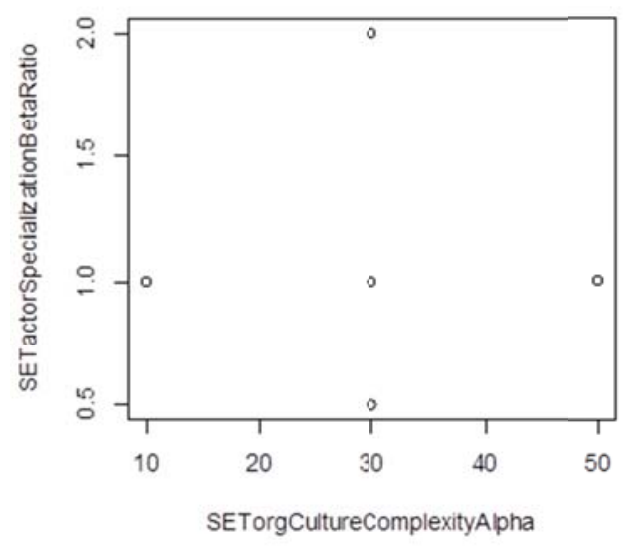

\subsubsection{Rank-Ordering of Factors}

Since the experiments are designed and carried out in an identical manner, the slopes therefore are able to be compared to one another (Becker \& Wu, 2007); Table 5-1 shows a combined rank ordering of the constructs according to the absolute-value of the coefficient.

Looking at the table, several observations can be made, which will be qualitatively interpreted in Chapter 6 Qualitative Results:

1. The size of the organization (number of work units) highly and negatively affects performance

1. The degree of infrastructure support positively affects task performance to a large extent (relative to other factors in the study).

2. The size of the work teams (number of actors) positively affects the performance 
3. The work design of the immediate work unit (Team interdependence and actor specialization) is next in relevance

4. An individual's tendency to seek out information is positively correlated with performance

5. Remaining factors included in this study indicate that they play a relatively minor role in shaping the performance outcome.

6. An individual's willingness to share information, or not, has virtually no relationship with the performance of the group.

Table 5-1. Rank order of Beta coefficients (standardized) for independent factors, gathered from all experiments (Absolute value; ordered greatest to least).

\begin{tabular}{|c|l|l|}
\hline $\begin{array}{c}\text { Type } \\
\text { (Structural /Motivational) }\end{array}$ & \multicolumn{1}{|c|}{ Construct } & \multicolumn{1}{c|}{ Beta Coefficient (Alpha / Beta) } \\
\hline S & Organizational Complexity & -18.0757 \\
\hline S & Infrastructure Support & $6.2712 / 4.8932$ \\
\hline S & Team complexity & $0.7112 / 0.2058$ \\
\hline S & Team Interdependence & $0.255 / 0.176$ \\
\hline S & Actor Specialization & $0.155 / 0.122$ \\
\hline M & Willingness to Seek & $0.126 / 0.093$ \\
\hline S & Culture Complexity Org & $-0.061 /-0.029$ \\
\hline S & Cultural Complexity Team & $-0.056 /-0.018$ \\
\hline M & Task Focus & $0.033(\mathrm{H}): 0.024(\mathrm{M}) / 0.047(\mathrm{H}): 0.051(\mathrm{M})$ \\
\hline M & Culture Outreach & $-0.005 /-0.006$ \\
\hline M & Willingness to Share & $0.0001 / 0.00004$ \\
\hline
\end{tabular}

\subsubsection{Structural Factors Outweigh Cultural}

An examination of the rank order list (Table 5-1) along with the classification of each factor indicates that as a group, structural factors have a stronger relationship-relative to cultural factors-with the post-merger performance outcome. Performing a test of difference between means on the beta coefficients (using the Alpha values only) according to the 
classification of the factor (Table 5-2), indicates that the two factors statistically do differ; thus a hypothesis that structural factors are of greater relevance than cultural factors cannot be rejected statistically.

Table 5-2. Z-Test: Two Sample for Means.

\begin{tabular}{lcc}
\hline & Structure & Cultural \\
\hline Mean & 3.655 & 0.041 \\
Known Variance & 45 & 0.003 \\
Observations & 7 & 4 \\
Hypothesized Mean & 0 & \\
$\begin{array}{l}\text { Difference } \\
z\end{array}$ & 1.425 & \\
P $(Z<=z)$ one-tail & 0.077 & \\
$z$ Critical one-tail & 1.645 & \\
\hline
\end{tabular}

This analysis indicates that structural characteristics affect the post-merger organizational performance outcome more so than do the cultural characteristics.

\subsection{Validation}

The Construct model has been validated numerous times (Carley, 1990; Carley \& Hill, 2001; Carley \& Krackhardt, 1996; Schreiber \& Carley, 2004b, 2007), and used extensively for organizational research; so it is deemed reliable and correct. An inspection of the trajectory outcome variables meets the validity face test, as in every case the monotonic behavior of the outcome variables was realized, as expected. More in-depth discussion on validation is provided in Section 7.5 Limitations and Section 7.8 Future Work. 


\section{CHAPTER 6: QUALITATIVE FINDINGS}

This chapter provides both a restatement of the constituent studies' findings, and an all-inclusive, combined synthesis of those finding, which provides perspectives not accessible looking any individual article. Before delving into the qualitative findings there are several decidedly significant clarifications that must be presented so that the reader does not misinterpret the discussion; these clarifying points are presented next.

\subsection{Clarifications}

Below, two clarifying points are presented to establish the context within which the qualitative discussion should be understood. Since this study is conducted in a virtual laboratory, clarification of these points is important and these may be construed as limitations, but are basically consequences of purposeful design decisions.

\subsubsection{Fixed Characteristics}

Organizations and individuals change over time as a normal progress of following a stressful event such as a merger. However, this study locks in the characteristics of NewCo based on the original characteristics of Alpha and Beta, as they were prior to the consummation of the merger deal. In real-world the announcement of the merger, for example, can greatly affect the cultural response of the individuals involved and even reverse their motivations, e.g., an individual may be energized by a merger occurring which could significantly change their perception and thus their motivation for sharing information, one way or the other. Moreover, this study does not recognize that an integration manager may restructure the NewCo in a manner that differs from either Alpha or Beta.

\subsubsection{Comparative Analysis}

Because of the abstractness of the computational models, the results of the experiments have limited use if applied to a specific merger scenario in isolation. Instead, the results are highly valued in a comparative analysis setting — comparing two different scenarios 
for the same merger, or comparing two different potential merger targets for a same acquirer. More extensive explanation of how to use the results in an applied setting - along with illustrative examples_-is provided in Chapter 7 Conclusions.

\subsection{Synopsis of Constituent Studies}

Each of the principal studies concludes with qualitative conclusions; these are repeated below:

\subsubsection{Study One}

"Post-Merger Knowledge Transfer: Examining Antecedent Structural Complexity", established five points:

(1) Organizations with lower organization-level complexity will make greater, more noticeable progress in task knowledge diffusion early-on in the post-merger integration period.

(2) Team-level complexity has a dynamic characteristically similar to that of organization-level complexity.

(3) The relative difference in team-level complexity between the two original organizations has minimal effect on the task-knowledge transfer rate.

(4) The level of infrastructure support positively affects the speed to which the organization reaches its task-knowledge saturation point.

(5) The relative difference in infrastructure support between the two original organizations has minimal effect early on in the integration process, but becomes more prominent in later stages of the process. 


\subsubsection{Study Two}

"Post-Merger Integration: Effects of Task Structure and Motivation to Exchange Information on Organization Performance" established three points:

(1) Managers may safely not be overly concerned about motivating the member of the acquired organization to share information with members of the acquiring organization,

(2) An organization with lots of interdependent work-teams may require less attention during the post-merger integration than an organization made up of unconnected work groups, and

(3) It may be just as effective to focus on information exchange only to a point of critical mass rather than attempting to attend to all members of the combined organization; the outcome may be just as positive.

\subsubsection{Study Three}

“The Effects of Legacy Organization Culture on Post-Merger Integration" established three points:

(1) The more complex the culture, the greater the time for post-merger organizational and team cultural integration to complete, while simultaneously task-performance suffers as well.

(2) The inclination for an organization to energetically share their culture with another group does not immensely impact the speed of diffusion of cultural or task knowledge, which is counter-intuitive.

(3) High levels of task focus in a culture can hinder culture-related diffusion, though performance is positively correlated with this characteristic. 
Organizational characteristics of culture-outreach and task-focus are not simple factors, and there may be an important interaction between these two that need to be studied more closely and modeled with more precision (see Section 5.3).

\subsection{Combined Findings}

\subsubsection{Rank-Ordering of Factors}

Below is a restatement of the combined quantitative findings presented in the previous chapter with qualitative comments added indicating the following numerous conclusions:

1. The size of the organization (number of work units) highly and negatively affects performance

a. Increases the amount of informational noise the individual takes in that does not directly improve task performance

2. Increased infrastructure support positively affects task performance to a large extent (relative to other factors in the study).

a. There is less knowledge that must pass between individuals

3. The size of the work units positively affects the performance

a. There are more individuals in close proximity that have information relevant to an individual's work task

4. The work design of the immediate work unit (Team interdependence and actor specialization) is next in relevance

5. An individual's tendency to seek out information is positively correlated with performance

6. Remaining factors included in this study indicate that they play a relatively minor role in shaping the performance outcome.

a. The complexity of the culture(s), both organizational and team level hinders task performance.

b. Task focus, thus disregarding cultural information thus learning about the other culture does not greatly hinder the performance, though this construct does not involve the ability to understand and communicate, etc. 
c. Making specific effort to teach others about aspects your own culture does slow down performance increases

7. An individual's willingness to share information, or not, has virtually no relationship with the performance of the group.

a. Individuals simply find other avenues to get the information they need.

\subsubsection{Structural Factors Outweigh the Cultural}

This simple analysis in general terms, establishes that structural characteristics affect the organizational performance outcome more so that cultural factors. This finding is congruent and support of research that emphasizes organizational structure (e.g., Burton \& Obel, 1984; Sydow \& Windeler, 1998; Uzzi, 1997) and is in stark contrast with culturallyfocus researchers (e.g., Bunderson \& Sutcliffe, 2002; Jehn \& Shah, 1997; Lin, 2007; MartinCruz, Martin-Perez, \& Trevilla-Cantero, 2009; Martin-Perez, Martin-Cruz, \& EstradaVaquero, 2012; Miranda \& Saunders, 2003).

\subsection{Validation}

The findings and conclusions of the above qualitative analysis are reasoned to meet the scrutiny of a face validity test by investigating real-world examples. There are numerous non-simulated situations that support the above qualitative findings. For one, looking outside the world of business-oriented organizations, but an organization just as any other, the integration of military services from differing countries in the Iraq and Afghanistan wars, is a strong case in point. The collation forces from The United States of America, the United Kingdom, and Australia (to name a few), successfully integrated into a distinct and unified performing organization - regardless of their different cultures - that were successful in their mission. Clearly, the various militaries melded into a single cohesive that was as focused on the task as any progressive business organization. Another military example occurred during the American Civil War: Blacks successfully fought beside Whites with little senior military leadership attention to the cultural differences between the two culturally different groups. To lend further support from the opposite view is the poor performance of the Americans in the Vietnam War: the American soldiers were fraught with cultural distractions leading to the 
feuding between Blacks and Whites which just could not integrate in the war trenches; this war was effectively lost.

A business organization example, where the focus on the task led to a successful integration, could be found in the 1997 merger of Morgan Stanley \& Co. and Dean Witter \& Co. In this case, the cultural integration of the two vastly different cultures was secondary to the performance of the merger. This author personally witnessed this strategy playing out over several years of working for the combined company. The senior management of the combined company first talked of the business benefits of the combination and spoke consistently about achieving corporate objectives and very little mention was made of the immense cultural differences between the two financial firms. This approach is still the operational mode of the company, which is now thriving financially from the combination first started nearly two decades ago.

Finally, consistent with the findings that a focus on the work structure is a more effective approach to integration management, there is the frequent success of conglomerate mergers which are, by definition and by design, less focused on organization integration and almost entirely focused on the primary task of producing business results; conglomerate mergers tend to be more successful financially and tend to have fewer debilitating cultural integration problems.

From the perspective of computational modeling and the use of the informationprocessing view of the organization as cornerstones to this study, previous research has demonstrated that these approaches have merit and are effective in formulating theory. A ground-breaking example is the Virtual Design Team (VDT) framework (Jin \& Levitt, 1996) which is consistent with the computational and information-centric approaches taken herein, and has been extensively validated (Levitt, Orr, \& Nissen, 2005). The VDT model is a wellaccepted by the scholarly community for over two decades (Levitt, 2004) as corresponding with actual behaviors in organization and thus is a productive computational model of organization behavior (see Wong \& Burton, 2000), just as the CONSTRUCT model is. 


\section{CHAPTER 7: CONCLUSIONS}

This study has two distinct, science-based deliverables: (a) the description of a qualitative A Behavioral Theory of the Merger, and (b) the presentation of a quantitative postmerger integration forecasting tool. The qualitative theory is expounded below, followed by a presentation of the quantitative forecasting tool. Afterwards, several practical ideas of how to apply the theory and tool to research and practice are put forth. This chapter continues with a presentation of the limitations of this study, suggestions for future work, an executive summary, and a formal, Research Funding Acknowledgements Section.

\subsection{A Behavioral Theory of the Merger}

The theory is grounded on the premise that information exchange-or, considered from a higher level, organization learning - is fundamental to the social integration process. Moreover, information exchange is essential to the development of constructive team performance. As presented earlier, scholarly literature is supportive of both of these principles. Indeed, effective managers are those that communicate well with essential others (see Appendix B for more on this perspective). With these two underlying principles in mind and given the experiments conducted in this study that drive the findings, there are two central facets of the Behavioral Theory of the Merger:

I) In the post-merger integration period, features relating to the structure of the work supersede, in their impact, those features that are cultural related, when it comes to the performance of the organization. The design of the work and the assignment of the task have greater influence on the performance outcome than do the cultural aspects of the organization. This suggests that scholars are being led astray by the increasing attention on cultural issues during post-merger integration. Indeed, practitioners often blame cultural differences for the failure of the integration, and scholars follow suit by prudently investigating culture. $A$ Behavioral Theory of the Merger offers that scholars and practitioners have been misdirected and that the management-controlled work structure may actually be more effectual and thus is actually to blame for integration failure; problematic, pre-existing cultures may simply be 
an undeserving scapegoat. This theory implies that management-controlled work structure can overcome cultural issues.

This conclusion is supported by the quantitative results presented in Section 5.3.1 and 5.3.2, where the rank-ordering of the complete set of thirteen organizational characteristics in these experiments were presented and analyzed collectively. It was found that nearly all seven structural features ranked higher than the cultural features - there was only one exception, willingness to seek (see Table 5-1). Statistical analysis (two-sample means t-test) showed that there is a reasonable expectation that there is indeed a difference $(p<0.05)$ between the structure-based features and the culture-based features according to these simulation experiments. The standardized coefficient for structure was 3.6 and for culture was 0.04 , indicating that structure has a stronger effect on the integration process, both in terms of organization performance and culture integration. Moreover, this conclusion is supported in conventional organization research programs-focused on organizational design and structure - conducted by scholars such as Burton and Obel (1984, 1998), Galbraith (1974, 1977), Burton, Eriksen, Hakonsson, and Snow (2006), and Gerloff (1985).

II) This theory also finds that during the post-merger integration period, there is an awkward competition for the limited communications capacity, or bandwidth, that individuals and groups are bound to. A Behavioral Theory of the Merger suggests that cultural differences do indeed create issue with organization performance, not because of the cultural behavior and conflicts per se, but because of the reduction of task-related learning, or information flow, that flows through the communications pipeline this reduction is caused by a limited communications capacity. This study finds that when there are cultural differences, actors communicate with one another by passing information about the problematic, cultural differences instead of communicating performance-critical, task-related information. This reduction in task-related information transfer slows down the organizational learning and thus hinders the progress of increasing organization performance.

This conclusion is supported by the holistic interpretation of the qualitative findings presented in Chapter 6 in combination with the quantitative findings put forth in Chapter 7. 
Specifically, culture complexity, both at the organization- and team-level negatively affected both organizational task performance and cultural integration (organization and team levels), as does cultural outreach, while task-focus positively affects both task performance and cultural integration (organization and team levels). This negative affect of cultural complexity and cultural outreach, coupled with the positive affect of task focus, and a detailed review of the underlying processes in a social and information-processing view of the organization, lead to this interpretation that the shared (task and culture information) information-exchange bandwidth is being overtaken by the volume of cultural information being exchanged; thus, pushing out available space for the task-oriented infomation. Moreover, this aspect of the theory is supported in conventional organization research programs - focused on the information processing view of the organization and the social information processing approach to organizational phenomena — conducted by scholars such as Galbraith (1974, 1977), Tushman and Nadler (1978), Daft, and Macintosh (1981), Rice and Aydin (1991), and Salancik and Pfeffer (1978).

These two facets making up the Behavioral Theory of the Merger reinforce to a manger that information exchange us an essential process in the post-merger integration period. It is an integral dimension of designing effective work structures and also it is a boundary constraint in organizational learning and thus in cultural integration. Indeed information exchange is foremost to this theory and to management practice in general.

\subsection{A Forecasting Tool}

The computational models developed from this study can serve as a forecasting tool in real-world situations, though with its application should be limited to very strict circumstances and conditions. A simple and practical example application of the model is to compare the merging of two small businesses, say two pizza shops. Alpha Pizza is choosing between a merger with Beta Pizza or with Charlie Pizza. Beta Pizza has 5 employees and Charlie Pizza has 10 employees. All other aspects, relative to Alpha Pizza, of Beta Pizza and Charlie Pizza are functionally identical. Applying the model from Study One, the coefficient for Alpha to Beta Agent Per Team Ratio is $-0.02 \%$, so I can expect post-merger integration 
performance for knowledge-unique tasks of Alpha-Charlie Pizza to trail Alpha-Beta Pizza by $(2.0 \times-0.02 \%=-0.04 \%)$ during the integration stage. The overall difference of performance (all else being equal) would be $-0.04 \%$ based on and due only on the size difference of Beta Pizza (5 employees) and Charlie Pizza (10 employees). Applying this to expected revenue of, say, US $\$ 100,000$ per year, the difference in annual revenue could be around US $\$ 40$. The takeaway could very well be that the size difference should not materially affect the decision of merging with Beta Pizza or Charlie Pizza.

\subsection{Applying the Theory to Practice}

In practice, A Behavioral Theory of the Merger can be applied in numerous ways, each according to the characteristics of the specific situation. Broadly, for a more rapid postmerger integration result, managers are advised to concentrate greater attention on the structure of the task and work teams, more so than emphasizing the organization's cultural differences and accompanying cultural issues. Managers should focus more of their attention on the task-related aspects of the organization; in short, structure the work effectively and focus subordinates' attention on the work to be done and performing well, rather than directing their attention to cultural integration. Doing so enables actors to focus their attention and their communications energy on the tasks to be accomplished, rather than sharing how one group differs or conflicts from the other with communicative counterparts. Moreover, any work-performance success may possibly carry over to more rapid cultural integration as the actors likely will socially bond faster as a result of the positive performance feedback. Regardless of culture group of the participants, managers should encourage subordinates to focus on accomplishing the work task and managers should focus on structuring the organization in a manner that is oriented directly to task-performance. This research finds that it is the structure of the organization - the manner in which the group is organized to perform-is paramount.

As a contrived example, in the case of an airline merger, managers might require pilot assignments to be structured so that the pilot and the co-pilot are each from the two premerger organizations. Given pilots' profession-trained attention to safety and performance, 
the two pilots would inherently prioritize their task over attention cultural differences. Furthermore, equally mixing the cabin crew would serve the same purpose and positive outcome. The inherent professionalism of the pilots and cabin attendants would override their cultural differences as they are highly-skilled and dedicated professionals self-expected to and trained to work and communicate as a coordinated team, regardless of their cultural and organizational origin. The Behavioral Theory of the Merger suggests that correctly structuring the work of the organization un such a manner will result in a more rapid integration-versus just keeping the original teams together, or separated from the other culture. One might think of this as forced integration; a progressive strategy, rather similar in the approach and along the lines of how America advanced the resolution of the race-issues of the 1960's.

Applying this Behavioral Theory of the Merger, of course, is not always assured to be the most effective approach to post-merger integration or performance, as situational and environmental circumstances can unsettle this theory and therefor affect the effectiveness of the approach; managers should not blindly apply this, or any, management theory without prudent situational attention, informed judgment and constant re-evaluation.

As an adjunct to the primary organizational event under study in this research-postmerger integration - the qualitative findings further inform managers on managing cultural conflict or issues in the business-as-usual situation, that outside of a merger event. In a nonmerger scenario, managers are best advised to focus more so on the structure of the task over and above a focus on cultural conflict. In a non-merger scenario, employees would not be sorting out group-level cultural differences and thus be distracted in their task communications; actors' communication and most actors' attention would be focused on task performance rather than disruptive cultural conflict. Indeed, conflict arises but such conflict would likely be limited to individual differences rather than systemic and widespread difference as can happen in the case or an organizational merger. One interesting situation for consideration would be the specific circumstance of a labor union formation in a nonunionized organization; in this situation an organization was previously unified but then selfdivided into conflicting fractions. 


\subsection{Contributions}

Summarizing the above conclusions, the principal discovery arising from this study is that there is clear evidence that structural aspects of the merging organizations have greater influence on the post-merger integration performance of the organization that cultural aspects; this scientific evidence contradicts what the common post-merger thinking is. This suggests that a well-structured organization may possibly overcome cultural issues, at some level.

From the substantive perspective, this study provides a basis for developing fresh theory on the dynamics of the integration process. This research takes an attribute-based view of the merger integration and takes a look at the phenomenon using an extraordinary number of variables and data points that are not available in other post-merger integration studies (Datta \& Grant, 1990; Hitt, Harrison, Ireland, \& Best, 1998). Moreover, this research unifies previous research from several separate scholarly streams beyond that specific to post-merger integration. The constituent experiments in this study provide strong evidence about the behavior dynamics of the merger from the perspective of the integration process with fidelity of data previously unseen. The quantitative models developed provide a rich understanding of the integration process and provide a strong basis for targeted empirical-based post-merger integration research

Moreover, from the methodological perspective, this research is first known published study to fully utilize computational modeling techniques for the sole purpose of investigating broad-based merger integration dynamics. It produced the first database of fully tractable metrics about the phenomena at a level of detail never before captured and with precision unavailable previously. The theoretic outcomes of this study are supported by an enormous amount data samples at levels never before available to earlier merger integration researchers.

\subsection{Limitations}

While computational models and simulations are an invaluable tool in theory development and testing, any findings should ultimately be confirmed by empirical case study. There are several aspects of organizational behavior that are not captured in the 
Construct model--for example, double-loop learning, actor incentive and reward systems (Burton \& Obel, 1984) - until confirmed multiple times, the findings from this research should not be blindly construed as being necessarily predictive (DeGreene, 1973), at this stage. Moreover, the critical issue of trust and its relationship with organization performance (Prietula \& Carley, 1999) is not addressed in the model. Furthermore, organizational unlearning (Tsang \& Zahra, 2008), both from perspective of knowledge transfer or cultural transmission, is not implemented. There are many more behavioral aspects of organizational behavior that are not captured in the model should be identified as limitations to the study from numerous other perspectives not taken in this research design.

In the combined meta-analysis of the three studies, since not all possible variables are included in the study, there may very well be antecedent characteristics that have an even stronger relationship with the post-merger integration outcomes than those involved in the study. While, this study does not necessarily aim to test all factors, the findings of this study must be understood in the context of the antecedent characteristics involved only.

Critically, this study does not account for management's integration strategy that often accompanies a merger. Although, some real-world managers do indeed take a laissez faire approach to integration management--purposely or otherwise--, herein the effect of the how the integration process is managed is left unexplored and therefore limits the applicability of the study from some operational perspectives. However, this study can adequately be considered a theoretical experiment and one in which a baseline scenario is established for later and more complex research and investigations that may include integration management strategies into the process.

This study is based on an agent-based computational model, specifically CONSTRUCT. The behaviors of the agents (See Appendix A, Section A.4) in the CONSTRUCT model reflect rationality. However, in A Behavioral Theory of the Firm, Cyert and March (1963) demonstrated that bounded rationality affects organization-level behaviors in a meaningful way. Indeed, the models employed in this study offer rational actors, thus the abstractness of the simulation is without this important component of real-world decision- 
making and behavior. Carley (2002a) points out that actor rationality is an important dimension in in computational organization theory, as well as, in the broader field of sociology (Carley, 1994). Previous research has been conducted that implements bounded rationality agents; for example, Arthur (1991) successfully implemented the rational agent in an economic simulation study. Including this feature in future work may prove to be fruitful and the results in the post-merger integration period could very well differ with such a feature active in the simulation. In this study, actors are assumed to use all knowledge available to them at all times, which is an improbable characteristic of actor behavior. The effect of this limitation is that actors will not affect the behavior of other actors decision-making, for example. The influence of others is implemented in CONSTRUCT through the process of information exchange, however, deviate decision-making or behaviors that can be influenced socially and thus spread throughout a social group which can happen in real-world situations are not possible in this model. Such deviate behaviors spreading through a group can likely affect the outcomes in a dramatic way.

Another limitation of this study is that personal trust is limited in the computational model; it is merely represented as a characteristic of willingness to seek in the experiments as a lightweight proxy for trust. Furthermore, organizational unlearning (Tsang \& Zahra, 2008), both from perspective of knowledge transfer or cultural transmission, is not implemented. Trust and unlearning are not directly represented in this study's model and should be identified as a limitation to the study and an opportunity for future work (see below).

\subsection{Future Work}

Specific future work can be identified to further refine these initial-research findings underlying A Behavioral Theory of the Merger. First, the next work to be done is to address the external validity of the results by extensively field-test the quantitative models - both to provide feedback and further inform the qualitative theory and to calibrate the quantitative models. To field-test The Theory, the researcher will need to overcome the problem of the structural challenge of procuring access to real-world cases of organizational mergers. A computer-based social simulation, such as in this study, can benefit from a field or laboratory 
test using actual human subjects, or real-world organizational cases, et cetera; of course, this study is no exception. The idealized airline merger scenario put forth in Section 7.2 (see Apply the Results) may also serve as a possible real-world, field test of the findings herein. By systematically manipulating and controlling the proportions of cabin attendants from the two original organizations for numerous flights and: (a) measuring the service feedback from passengers from numerous flights, (b) conducting blind observation by a researcher disguised as a passenger (to quantify the interaction dynamics of the crew), and (c) surveying the cabincrew subjects, a particular forecast and projection from the Behavioral Theory of the Merger - for this one particular airline merger - can be evaluated and calibrated to this realworld scenarios with some degree of favorable confidence.

Second - a much more feasible future work activity_, is to re-test the consolidated findings (see Chapter 5) by formulating a study that consists of running additional simulationbased experiments where the variables are combined in a random fashion and simulations run on those variable combinations. Such a procedure would help to better calibrate the quantitative model and serve to re-confirm the findings put forth in following the above procedures. The methodology would be entirely consistent to the methods carried out in this study.

Third, the primary finding of this study - that structure is more important than motivation-would benefit from concentrated research focused solely on that finding, to support, or disprove the overarching concept. A human laboratory experiment that would address this specifically would be complex to design and carry out effectively. Fourthly, answering the question of how to integrate the findings of this study-recall that the models focus only on the knowledge differences - with the dimensions of the knowledge that are the same in the two merging organizations; need to understand how to combine the pre-shard knowledge with the post-shared knowledge to reach a comprehensive outcome metric; for example, forecasting the true and complete organization performance rather than only that pertaining to the knowledge differenced performance. 
Fourth, one aspect of mergers of interest and could be future work, from an academic and practice-research perspective, is the period of time between the merger agreement and the consummation of the merger. Legally, in the case of a corporate merger, particularly involving industry competitors, the two firms are legally forbidden to work together or collude with one another prior to the consummation of the merger. This restriction is in place because should the merger be cancelled and thus not consummated for any reason, such cooperation would be considered collusion which is illegal in most circumstances. Competitors are legally forbidden to work together and share corporate secrets. Moreover, any integration efforts pre-merger would be highly problematic should the merger agreement not result in an actual consummation of the merger. However, not all mergers involve legallybound corporations: two Little League baseball teams can merge and can consequently engage into some pre-merger conditioning for the pending integration. To study this preconditioning stage, the Robbers Cave experiment (Sherif, Harvey, White, Hood, \& Sherif, 1961) could be reproduced with a pre-merger phase set-up such that the boys could be preconditioned for the post-merger integration by suggesting positive aspects of the other group.

Finally, interpersonal and group trust is an important and complex dimension of information exchange and organizational dynamics (Brower, Schooman, \& Tan, 2000) and it follows that it must have some effect on post-merger integration. This study utilizes the characteristics of willingness to seek in the experiments as a lightweight proxy for trust; however, this is merely one dimension of the complex phenomenon (Jeffries, 2002; Jeffries \& Becker, 2008). Trust is essential in group cooperation, exchange, and stability (Hosmer, 1995) and has a significant role in teamwork (Jones \& George, 1998). Moreover, the critical issue of trust and its relationship with organization performance (Prietula \& Carley, 1999) is not addressed in the model. Furthermore, organizational unlearning (Tsang \& Zahra, 2008), both from perspective of knowledge transfer or cultural transmission, is not implemented. Trust is not directly represented in this study's model and should be identified as a limitation to the study and an opportunity for future work. 


\subsection{Executive Summary}

This study advances the theoretic understanding of post-merger integration phenomenon. It formulates a behavioral theory of the merger: it focuses on the dynamics of the post-merger integration process, in particular the human systems. Virtual experiments were conducted to study the subtleties of information exchange within a newly formed organization following the consummation of organizational merger.

This study provided clear evidence that structural factors, e.g., work unit task assignment and the number of work teams, are more relevant to the post-merger integration outcome than cultural factors, e.g., willingness to share information and task focus of the individuals. This suggests that integration managers can influence the outcome of the postmerger integration period more so by downplaying the attention to motivating employees and instead focusing greater attention on crafting the proper work structure of the organizational units.

The strength of the methodology employed in this study suggests that these models have robust and theoretically-predictive power to quantitatively forecast dynamics in realworld mergers; this must be confirmed through extensive laboratory and case-based study. This research facilitates additional development of detailed and reliable estimators and thus advances the reliable forecasts which may be invaluable in real-world merger situations.

\subsection{Research Funding Acknowledgements}

This work is supported in part by the Air Force Office of Sponsored Research (AFOSR) (FA9550-11-1-0179) for multi-level modeling, the Office of Naval Research (ONR) (MMT N00014-06-1-0104) for dynamics, and the National Science Foundation IGERT in CASOS (NSF) (DGE 997276). Additional support came from Carnegie Mellon University's Center for Computational Analysis of Social and Organizational Systems. The views and conclusions contained in this document are those of the authors and should not be interpreted as representing the official policies, either expressed or implied, of the Air Force 
Office of Sponsored Research, the Office of Naval Research, the National Science Foundation, or the U.S. government. 
7-13 



\section{CHAPTER 8: REFERENCES}

Almeida, P., \& Kogut, B. (1999). Localization of knowledge and the mobility of engineers in regional networks. Management Science, 45, 905-917.

Ardichvili, A., Page, V., \& Wentling, T. (2003). Motivation and barriers to participation in virtual knowledge-sharing communities of practice. Journal of Knowledge Management, 7, 64-77.

Argote, L. (1999). Organizational learning: Creating, retaining, and transferring knowledge. Norwell, MA: Kluwer.

Argote, L., \& Ingram, P. (2000). Knowledge transfer: A basis for competitive advantage in firms. Organizational Behavior and Human Decision Processes, 82, 150-169.

Argote, L., Gruenfeld, D. H., \& Naquin, C. (2000). Group learning in organizational. In M. E. Turner (Ed.), Groups at work: Advances in theory and research (pp. 369-411). Hillsdale, NJ: Erlbaum.

Arthur, W. B. (1991). Designing economic agents that act like human agents: A behavioral approach to bounded rationality. American Economic Review Papers and Proceedings, 81, 353-359.

Ashford, S. J., \& Taylor, M. S. (1990). Adaptation to work transitions: An integrative approach. In G. R. Ferris and K. M. Rowland (Eds.), Research in personnel and human resource management, Vol. 8 (pp. 1-39). Greenwich, CT: JAI Press.

Austin, J. R. (2003). Transactive memory in organizational groups: The effects of content, consensus, specialization, and accuracy on group performance. Journal of Applied Psychology, 88, 866-878.

Axelrod, R. (1986). An evolutionary approach to norms. The American Political Science Review, 80(4), 1095-1111.

Bankes, S., \& Lempert, R. (2004). Robust reasoning with agent-based modeling. Nonlinear Dynamics, Psychology, and Life Sciences, 8, 259-277.

Barrett, S., \& Konsynski, B. (1982). Inter-organization information sharing systems. MIS Quarterly, 6(4), 93-105.

Barrick, M. R., Bradley, B. H., Colbert, A. E. (2007). The moderating role of top management team interdependence: Implications for real teams and working groups. Academy of Management Journal, 50(3), 544-557.

Bass, B. M. (1967). Social behaviour and the orientation inventory: A review. Psychological Bulletin. 68, 260-292.

Baum, J. A. C., \& Ingram, P. 1998. Survival-enhancing learning in the Manhattan hotel industry 1898-1980. Management Science, 44, 996-1016 
Becker, M. C., \& Knudsen, M. P. (2006). Intra and inter-organizational knowledge transfer processes: Identifying the missing links. Danish Research Unit of Industrial Dynamics, DRUID Working Paper No. 06-32.

Becker, B. J., \& Wu, M-J. (2007). The synthesis of regression slopes in meta-analysis. Statistical Science, 22(3), 414-429.

Becker, M. H. (1970). Sociometric location and innovativeness: Reformulation and extension of the diffusion model. American Sociological Review, 35, 267-282.

Benito, G. R., \& Gripsrud, G. (1992). The Expansion of Foreign Direct Investments: Discrete Rational Location Choices or a Cultural Learning Process? Journal of International Business Studies, 23(3), 461-476.

Bert, A., MacDonald, T., \& Herd, T. (2003). Two merger integration imperatives: Urgency and execution. Strategy \&Leadership, 31(3), 42-49.

Bettencourt, B., Brewer, M. B., Croak, M. R., \& Miller, N. (1992). Cooperation and the reduction of intergroup bias: The role of reward structure and social orientation. Journal of Experimental Social Psychology, 28(4), 301-319.

Bierly, P., \& Chakrabarti, A. (1996). Generic knowledge strategies in the U.S. pharmaceutical industry. Strategic Management Journal, 17, 123-135.

Birkinshaw J. M, Bresman, H., \& Hakanson, L. (2000). Managing the post-acquisition integration process: How the human integration and task integration processes interact to foster value creation. Journal of Management Studies, 37(3), 395-426.

Blau, P. M. (1970). A formal theory of differentiation in organizations. American Sociological Review, 35, 201-218.

Blumer, H. (1969). Symbolic interactionism: Perspective and method. Englewood Cliffs, NJ: Prentice-Hall.

Bock, G-W., \& Kim, Y-G. (2002). Breaking the myths of rewards: An exploratory study of attitudes about knowledge sharing. Information Resources Management Journal, $15(2), 14-21$.

Bock, G-W., Zmud, R. W., Kim, Y-G., \& Lee, J-N. (2005). Behavioral intention formation in knowledge sharing: Examining the roles of extrinsic motivators, social-psychological forces, and organizational climate. MIS Quarterly, 29, 87-111.

Boen, F., Vanbeselaere, N., \& Cool, M. (2006). Group status as a determinant of organizational identification after a takeover: A social identity perspective. Group Processes \& Intergroup Relations, 9, 547-560.

Boland, R. J., \& Tenkasi, R. V. (1995). Perspectives making and perspective taking in communities of knowing. Organization Science, 6, 350-372.

Borgatti, S. P., \& Cross, R. (2003). A relational view of information seeking and learning in social networks. Management Science, 49, 432-445. 
Bresman, H., Birkinshaw, J., \& Nobel, R. (1999). Knowledge transfer in international acquisitions. Journal of International Business Studies, 30, 439-462.

Brett, J. M., Feldman, D. C., \& Weingart, L. R. (1990). Feedback-seeking behavior of new hires and job changers. Journal of Management, 16, 737-749.

Brewer, M. B., \& Miller, N. (1988). Contact and cooperation. In Phyllis A. Katz and Dalmas A. Talyor (Eds.), Eliminating racism (pp. 315-326). New York: Springer.

Brower, H. H., Schooman, F. D., \& Tan, H. Ho. (2000). A model of relational leadership: The integration of trust and leader-member exchange. The Leadership Quarterly, 11(2), $227-250$.

Bruner, R. F. (2004). Applied mergers \& acquisitions. Hoboken, NJ: Wiley.

Bruner, R. F. (2005). Profiles of the outlying M\&A transactions, 1985 to 2000. http://dx.doi.org/10.2139/ssrn.681282

Bryson, J. (2003). Managing HRM risk in a merger. Employee Relations, 25, 14-30.

Bunderson, J. S., \& Sutcliffe, K. M. (2002). Comparing alternative conceptualizations of functional diversity in management teams: Process and performance effects. Academy of Management Journal, 45, 875-893.

Buono, A. F., \& Bowditch, J. L. (1989). The human side of mergers and acquisitions: Managing collisions between people, cultures, and organizations. San Francisco: Jossey-Bass.

Buono, A. F., Bowditch, J. L., \& Lewis, J. W. (1985). When cultures collide: The anatomy of a merger. Human Relations, 38(5), 477-500.

Burt, R. S. (1973). The differential impact of social integration on participation in the diffusion of innovations. Social Science Research, 2, 125-144.

Burt, R. S. (1980). Innovation as a structural interest: Rethinking the impact of network position innovation adoption. Social Networks, 4, 337-355.

Burton, R. M. (1998). Validating and docking: An overview, summary and challenge. In M. Prietula, K. Carley, and L. Gasser, (Eds.), Simulating organizations: Computational models of institutions and groups (pp. 215-228). Cambridge, MA:AAAI/MIT Press.

Burton, R. M. (2003). Computational laboratories for organization science: Questions, validity and docking. Computational \& Mathematical Organization Theory, 9, 91108.

Burton, R. M., Eriksen, B., Hakonsson, D. D., Snow, C. C. (2006). Organization design: The evolving state-of-the-art. New York: Springer.

Burton, R. M., \& Obel, B. (1984). Designing efficient organizations: Modelling and experimentation. NY: Elsevier Science. 
Burton, R. M., \& Obel, B. (1995). The validity of computational models in organization science: From model realism to purpose of the model. Computational \& Mathematical Organization Theory, 1(1), 57-71.

Burton, R. M., \& Obel, B. (1998). Strategic organizational diagnosis and design: Developing theory for application (2nd ed.). Norwell, MA: Kluwer Academic Publishers.

Byrne, D. (1971). The attraction paradigm. New York: Academic Press

Capron, L. (1996). Mechanism of value creation in horizontal mergers and acquisitions: A test of efficiency, market power and resource-based arguments. Richard Ivey School of Business working paper, 96-23.

Carley, K. M. (1986). Knowledge acquisition as a social phenomenon. Instructional Science, $14,381-438$.

Carley, K. M. (1990). Group stability: Socio-cognitive approach . In A E. Lawler, B. Markovsky, C. Ridgeway, and H. Walker (Eds.), Advances in Group Processes (Vol. 7, pp. 1-44). Greenwich, CT: JAI Press.

Carley, K. M. (1991). A theory of group stability. American Sociological Review, 56(3), 331354. Reprinted in Organizational Networks Research, 2011, Martin Kilduff Diageo and Andrew V. Shipilov (Eds), Sage.

Carley, K. M. (1994). Sociology: Computational organization theory. Social Science Computer Review, 12(4), 611-624.

Carley, K. M. (2002a). Smart agents and organizations of the future. In Leah A. Lievrouw and Sonia Livingstone (Eds.), The handbook of new media, social shaping and consequences of ICTS (Ch 12, pp. 206-220). Thousand Oaks, CA: Sage

Carley, K. M. (2002b). Intra-organizational computation and complexity. In J. A. C. Baum (Ed.), The Blackwell companion to organizations (pp. 208-232). Malden, MA: Blackwell Publishers.

Carley, K. M. (2003). Dynamic network analysis. In Ronald Breiger, Kathleen M. Carley, and Philippa Pattison (Eds.), Dynamic social network modeling and analysis: Workshop summary and papers (pp. 133-145). Washington, DC: National Research Council.

Carley, K. M., Diesner, J., Reminga, J., \& Tsvetovat, M. (2007). Toward an interoperable dynamic network analysis toolkit. Decision Support Systems, 43, 1324-1347.

Carley, K. M. \& Frantz, T. L. (2009). Modeling organizational and individual decision making. In A. P. Sage \& W. B. Rouse (Eds.), Handbook of systems engineering and management (pp. 723-762). New York: Wiley.

Carley, K. M. \& Hill, V. (2001). Structural change and learning within organizations. In A. Lomi and E. R. Larsen (Eds.), Dynamics of organizations: Computational modeling and organizational theories (pp. 63-92). Menlo Park: MIT Press.

Carley, K. M., Martin, M. M., \& Hirshman, B. (2009). The etiology of social change. Topics in Cognitive Science, 1(4), 621-650. 
Carneiro, R. L. (1970). Scale analysis: Evolutionary sequences and the rating of cultures. In R. Naroll and R. Cohen (Eds.), A handbook of method in cultural anthropology (pp. 834-871). New York: Garden City Press.

Carroll, T., \& Burton, R. M. (2000). Organizations and Complexity: Searching for the edge of chaos. Computational \& Mathematical Organization Theory, 6(4), 319-337.

Case, D. O. (2007). Looking for information: A survey of research on information seeking, needs, and behavior (2nd ed). Amsterdam: Academic Press, Elsevier, Ltd.

Chaffee, S. (1991). Communication concepts 1: Explication. Newbury Park, CA: Sage Publications.

Chick, G. (1997). Cultural complexity: The concept and its measurement. Cross-cultural research, 31(4), 275-307.

Child, J. (1972). Organizational structure, environment and performance: The role of strategic choice. Sociology, 6(1), 1-22.

Clark, S. M., Gioia, D. A., Ketchen, Jr., D. J., \& Thomas, J. B. (2010). Transitional identity as a facilitator of organizational identity change during a merger. Administrative Science Quarterly, 55, 397-438.

Coleman, J. S., Katz, E., \& Menzel, H. (1957). The diffusion of an innovation among physicians. Sociometry, 20, 253-270.

Coleman, J. S., Katz, E., \& Menzel, H. (1966). Medical innovation: A diffusion study. Indianapolis: Bobbs-Merrill Co.

Connelly, C. E., \& Kelloway, E. K. (2003). Predictors of employees' perceptions of knowledge sharing cultures. Leadership \& Organization Development Journal, 24(5), 294-301.

Constant, D., Kiesler, S., \& Sproull, L. (1994). What's mine is ours, or is it? A study of attitudes about information sharing. Information Systems Research, 5, 400-421.

Cording, M., Christmann, P., \& King, D. R. (2008). Reducing casual ambiguity in acquisition integration: Intermediate goals as mediator of integration decisions and acquisition performance. Academy of Management Journal, 51, 744-767.

Cowan, R., \& Jonard, N. (2004). Network structure and the diffusion of knowledge. Journal of Economic Dynamics \& Control, 28, 1557-1575.

Crant, J. M. (2000). Proactive behavior in organizations. Journal of Management, 26, 435462.

Cremer, J. (1993). Corporate culture and shared knowledge. Industrial and Corporate Change, 2, 351-386.

Cyert, R. M., \& March, J. G. (1963). A behavioral theory of the firm. Oxford: Blackwell.

D’Andrade, R. (1981). The cultural part of cognition. Cognitive Science, 5, 179-195. 
D'Andrade, R. (1995). The development of cognitive anthropology. New York: Cambridge University Press.

D’Andrade, R., \& Strauss, C. (Eds., 1992). Human motives and cultural models. Cambridge: Cambridge University Press.

Daft, R. L., \& Macintosh, N. B. (1981). A tentative exploration into the amount and equivocality of information processing in organizational work units. Administrative Science Quarterly, 26, 207-224.

Dal Forno, A., \& Merlone, U. (2004). Personnel turnover in organizations: An agent-based simulation model. Nonlinear Dynamics, Psychology, and Life Sciences, 8, 205-230.

Darr, E., Argote, L., \& Epple, D. (1995). The acquisition, transfer, and depreciation of knowledge in service organizations: Productivity in franchises. Management Science, $41,1750-1762$.

Davis, M. S. (1973). Intimate relations. New York: Free Press.

De Dreu, C. K. W. (2007). Cooperative outcome interdependence, task reflexivity, and team effectiveness: A motivated information processing perspective. Journal of Applied Psychology, 92, 628-638.

de Vries, R. E., van den Hooff, B., \& de Ridder, J. A. (2006). Explaining knowledge sharing: The role of team communication styles, job satisfaction, and performance beliefs. Communication Research, 33, 115-135.

DeCarolis, D. M., \& Deeds, D. L. (1999). The impact of stocks and flows of organizational knowledge on firm performance: An empirical investigation of the biotechnology industry. Strategic Management Journal, 20, 953-968.

Deeker, D., Stokman, F., \& Franses, P. H. (2000). Broker positions in task-specific knowledge networks: Effects on perceived performance and role stressors in an account management system. ERIM Report Series Research in Management (ERS-2000-37MKT), Rotterdam School of Management, Erasmus University.

DeLong, D., \& Fahey, L. (2000). Diagnosing cultural barriers to knowledge management. Academy of Management Executive, 14, 113-127.

Denton, T. (2004). Cultural complexity revisited. Cross-Cultural Research, 38, 3-26.

DiMaggio, P., \& Useem, M. (1978). Cultural democracy in a period of cultural expansion: The social composition of arts audiences in the United States. Social Problems, 26, 179.

Dooley, K. J., \& Corman, S. R. (2004). Dynamic analysis of new streams: Institutional versus environmental effects. Nonlinear Dynamics, Psychology, and Life Sciences, 8, 403428.

Dooley, K. J., Corman, S. R., McPhee, R. D., \& Kuhn, T. (2003). Modeling high-resolution broadband discourse in complex adaptive systems. Nonlinear Dynamics, Psychology, and Life Sciences, 7, 61-86. 
Du, Rong, A., S., \& Ren, Yu. (2007). Relationship between knowledge sharing and performance: A survey in Xi'an, China. Expert Systems with Applications, 32, 38-46.

Duranti, A. (1997). Linguistic anthropology. New York: Cambridge University Press.

Eisenberger, R., Armeli, S., Rexwinkel, B., Lynch, P. D., \& Rhoades, L. (2001). Reciprocation of perceived organizational support. Journal of Applied Psychology, 86, 42-51.

Elliott, E. \& Kiel, L. D. (2004). Agent-based modeling in the social and behavioral sciences. Nonlinear Dynamics, Psychology, and Life Sciences, 8, 121-130.

Ellis, A. P. J., Hollenbeck, J. R., Ilgen, D. R., Porter, C. O. L. H., West, B. J., \& Moon, H. (2003). Team learning: Collectively connecting the dots. Journal of Applied Psychology, 88, 821-835.

El-Shinnawy, M., \& Markus, M. L. (1998). Acceptance of communication media in organizations: Richness or features? IEEE Transactions on Professional Communication, 41, 242-253.

Epple, D., Argote, L., \& Murphy, K., (1996). An empirical investigation of the microstructure of knowledge acquisition and transfer through learning by doing. Operations Research, 44, 77-86.

Epstein, M. J. (2004). The drivers of success in post-merger integration. Organizational Dynamics, 33(2), 174-189.

Frantz, T. L. (2012). A social network view of post-merger integration. In C. L. Cooper and S. Finkelstein (Eds.), Advances in mergers and acquisitions, Vol. 10. (pp. 161-176). Oxford, UK: JAI Press.

Frantz, T. L., \& Carley, K. M. (2009a). Agent-based modeling within a dynamic network. In S. Guastello, M. Koopmans, and D. Pincus (Eds.), Chaos and complexity in psychology: The theory of nonlinear dynamical systems (pp. 475-505). New York: Cambridge University Press.

Frantz, T. L., \& Carley, K. M. (2009b). Computationally modeling the effect of organizational complexity on post-merger integration. In C. L. Cooper and S. Finkelstein (Eds), Advances in mergers and acquisitions, Vol.8 (pp. 79-101). Oxford, UK: JAI Press

Frantz, T. L., \& Carley, K. M. (2010). Post-merger knowledge transfer: Examining antecedent structural complexity. In E. Tsui (Ed.), Proceedings of ICICKM 2010, The 7th International Conference on Intellectual Capital, Knowledge Management \& Organisational Learning. The Hong Kong Polytechnic University, Hong Kong, China 11-12 November 2010 (pp. 190-199). Hong Kong: Polytechnic University.

Galbraith, J. R. (1973). Designing complex organizations. Reading, MA: Addison-Wesley.

Galbraith, J. R. (1974). Organization design: an information processing view. Interfaces, 4(3), 28-36.

Galbraith, J. R. (1977). Organization design. Reading, MA: Addison-Wesley. 
Galpin, T. J., \& Herndon, M. (2010). The complete guide to mergers and acquisitions: Process tools to support $M \& A$ integration at every level. New York: John Wiley \& Sons.

Galpin, T. J., \& Robinson, D. E. (1997). Merger integration: The ultimate change management challenge. Mergers and Acquisitions, 31, 24-28.

Garud, Raghu, \& Kumaraswamy (2005). Vicious and virtuous circles in the management of knowledge: The case of Infosys Technologies. MIS Quarterly, 29, 9-33.

Gerloff, Edwin A. (1985). Organizational theory and design: A strategic approach to management. New York: McGraw-Hill.

Gigone, D., \& Hastie, R. (1993). The common knowledge effect: Information sharing and group judgment. Journal of Personality and Social Psychology, 65(5), 959.

Goh, S. C. (2002). Managing effective knowledge transfer: an integrative framework and some practice implications. Journal of Knowledge Management, 6, 23-30.

Granovetter, M. S. (1973). The strength of weak ties. American Journal of Sociology, 78, 1360-1380.

Granovetter, M. S. (1974). Getting a job: A study of contacts and careers. Cambridge: Harvard University Press.

Gruenfeld, D. H., Martorana, P. V., \& Fan, E. T. (2000). What do groups learn from their worldliest members? Direct and indirect influence in dynamic teams. Organizational Behavior and Human Decision Processes, 82, 45-59.

Guerin, S., \& Kunkle, D. (2004). Emergence of constraint in self-organizing systems. Nonlinear Dynamics, Psychology, and Life Sciences, 8, 131-146.

Gupta, A., \& Govindarajan, V. (2000). Knowledge flows within MNCs. Strategic Management Journal, 21, 473-96.

Hackman, J. R., \& Morris, C. G. (1975). Groups tasks, group interaction process, and group performance effectiveness: A review and proposed integration. In L. Berkowitz (Ed.), Advances in experimental social psychology, Vol. 9 (pp. 45-99). New York: Academic Press.

Hamel, G., Doz, Y. L., \& Prahalad, C. K. (1989). Collaborate with your competitors--and win. Harvard Business Review, 67(1), 133-139.

Hannerz, U. (1991). Cultural complexity. New York: Columbia University Press.

Hansen, M. (1999). The search-transfer problem: The role of weak ties in sharing knowledge across organization subunits. Administrative Science Quarterly, 44, 82-112.

Harrison, J. (2007). Why integration success eludes many buyers. Mergers and Acquisitions Journal, 42(3), 18-21. 
Heo, D., Yoo, Y. (2002). Knowledge sharing in post merger integration. Case Western Reserve University, USA. Sprouts: Working Papers on Information Systems, 2. Retrieved 22 August, 2011, http://sprouts.aisnet.org/2-18

Hinds, P., \& Kiesler, S. (1995).Communication across boundaries: Work, structure, and use of communication technologies in a large organization. Organization Science, 6, 373393.

Hirst, G., Van Knippenberg, D., \& Zhou, J. (2009). A cross-level perspective on employee creativity: Goal orientation, team learning behavior, and individual creativity. Academy of Management Journal, 52, 280-293.

Hofstede, G. (1983). The cultural relativity of organizational practices and theories. Journal of International Business Studies, 14(Fall), 75-89.

Hogg, M. A., \& Terry, D. J. (2000). Social identity and self-categorization processes in organizational context. Academy of Management Review, 25, 121-140.

Hosmer, L. T. (1995). Trust: The connecting link between organizational theory and philosophical ethics. Academy of Management Review, 20, 379-403.

Hsu, M-H., Ju, T. L., Yen, C-H., \& Chang, C-M. (2007). Knowledge sharing behavior in virtual communities: The relationship between trust, self-efficacy, and outcome expectations. International Journal of Human-Computer Studies, 65, 153-169

Iwanaga, S., \& Namatame, A. (2002). The complexity of collective decision. Nonlinear Dynamics, Psychology, and Life Sciences, 6, 137-158.

Janz, B. D., Colquitt, J. A., \& Noe, R. A. (1997). Knowledge worker team effectiveness: The role of autonomy, interdependence, team development, and contextual support variables. Personnel Psychology, 50, 877-904.

Jehn, K. A. (1995). A multimethod examination of the benefits and detriments of intragroup conflict. Administrative Science Quarterly, 40, 256-282.

Jehn, K. A., \& Shah, P. P. (1997). Interpersonal relationships and task performance: An examination of mediating processes in friendship and acquaintance groups. Journal of Personality and Social Psychology, 72, 775-790.

Jehn, K. A., Northcraft, G. B., \& Neale, M. A. (1999). Why differences make a difference: A field study of diversity, conflict, and performance in workgroups. Administrative Science Quarterly, 44, 741-763.

Jeffries, F. (2002). Subjective norms, dispositional trust, and initial trust development. Journal of Behavioral and Applied Management, 3(2), 129-139.

Jeffries, F., \& Becker, Thomas E. (2008). Trust, norms, and cooperation: Development and test of a simplified model. Journal of Institute of Behavioral and Applied Management, 9(3), 316-336.

Jin, Y., Levitt, R. E. (1996). The virtual design team: A computational model of project organizations. Computational \& Mathematical Organizational Theory, 2, 171-195. 
Jo, S. J. \& Joo, K. (2011). Knowledge sharing: The influences of learning organization culture, organization commitment, and organizational citizenship behaviors. Journal of Leadership \& Organizational Studies, 18, 353-364.

Jones, G. R. \& George, J. M. (1998). The experience and evolution of trust: Implications for cooperation and teamwork. Academy of Management Review, 23, 531-546.

Kane, A. A., Argote, L., \& Levine, J. M. (2005). Knowledge transfer between groups via personnel rotation: Effects of social identity and knowledge quality. Organizational Behavior and Human Decision Processes, 96, 56-71.

Kankanhalli, A., Tan, B. C. Y., \& Wei, K-K. (2005). Contributing knowledge to electronic knowledge repositories: An empirical investigation. MIS Quarterly, 29, 113-143.

Kaufer, D. S., \& Carley, K. M. (1993). Communication at a distance: The influence of print on sociocutural organization and change. Hillsdale, NJ: Lawrence Erlbaum Associates.

Keller, R. T. (1994). Technology-information processing fit and the performance of R\&D project groups: A test of contingency theory. Academy of Management Journal, 37, 167-179.

Keller-McNulty, S., Bellman, K. L., Carley, K. M., Davis, P. K., Ivanetich, R. et al. (2006). Defense modeling, simulation, and analysis: Meeting the challenge. Washington, DC: National Academy Press

Kimberly, J. R., \& Evanisko, M. J. (1981). Organizational innovation: The influence of individual, organizational, and contextual factors on hospital adoption of technological and administrative innovations. Academy of Management Journal, 24, 689-713.

King, A. (1999). Retrieving and transferring embodied data: Implications for the management of interdependence within organizations. Management Science, 45, 918-935.

Ko, D-G., Kirsch, L. J., \& King, W. R. (2005). Antecedents of knowledge transfer from consultants to clients in enterprise system implementations. MIS Quarterly, 29, 59-85.

Krackhardt, D. (1994). Graph theoretical dimensions of informal organizations. In Kathleen M. Carley and Michael Prietula (Eds.), Computational Organizational Theory (pp. 89111). Hillsdale, NJ: Lawrence Erlbaum Associates, Inc.

Krackhardt, D., \& Brass D. (1993). Intraorganizational networks: The micro side. In S. S. Wasserman and J. Galaskiewicz (Eds.), Advances in Social Network Analysis: Research in the Social and Behavioral Sciences (pp. 230-253). Thousand Oaks, CA: Sage.

Krackhardt, D., \& Carley, K. M. (1998). PCANS model of structure in organizations. In Proceedings of the 1998 International Symposium on Command and Control Research and Technology, June, Monterrey, CA (pp.113-119). Vienna, VA: Evidence Based Research.

Krackhardt, D., \& Hanson, J. R. (1993). Informal networks: The company behind the charts. Harvard Business Review, 71(4), 104-111. 
Kroeber, A., \& Kluckholm, C. (1952). Culture: A critical review of concepts and definitions. Papers of the Peabody Museum of American Archeology and Ethnology, 47, 1-223.

Larsson, R., Bengtsson, L., Henriksson, K., \& Sparks, J. (1998). The interorganizational learning dilemma: Collective knowledge development in strategic alliances. Organization Science, 9, 285-305.

Levitt, R. E. (2004). Computational modeling of organizations comes of age. Computational \& Mathematical Organization Theory, 10, 127-145.

Levitt, R. E., Orr, R. J., \& Nissen, M. (2005). Validating the virtual design team (VDT) computational modeling environment. The Collaboratory for Research on Global Projects, Working Paper \#25, 1-15. Available at: http://crgp.stanford.edu/publications/working_papers/WP25.pdf

Liang, D. W. (1994). The effects of top management team formation on firm performance and organizational effectiveness. Unpublished Doctoral Dissertation, Carnegie Mellon University, Pittsburgh, PA.

Lin, B-W., Hung, S-C., and Li, P-C. (2006). Mergers and acquisitions as a human resource strategy: Evidence from US banking firms. International Journal of Manpower, 27(2), 126-142.

Lin, H-F. (2006). Impact of organizational support on organizational intention to facilitate knowledge sharing. Knowledge Management Research \& Practice, 4, 26-35.

Lin, H-F. (2007). Effects of extrinsic and intrinsic motivation on employee knowledge sharing intentions. Journal of Information Science, 33(2), 135-149.

Lin, N. (1999). Building a network theory of social capital. Connections, 22, 28-51.

Lin, N., \& Burt, R. S. (1975). Differential effects of information channels in the process of innovation diffusion. Social Forces, 54, 256-274.

Lin, Z. (1994). Organizational performance -- Theory and reality. Doctoral Dissertation, Carnegie Mellon University, Pittsburgh, PA.

Lin, Z. (2000). Organizational restructuring and the impact of knowledge transfer. Journal of Mathematical Sociology, 24, 129-149.

Lin, Z., \& Carley, K. (2003). Designing stress resistant organizations: Computational theorizing and crisis applications. Boston, MA: Kluwer Academic Publishers.

Linton, R. (1940). Acculturation in seven American Indian tribes. New York: AppletonCentury Company.

Lundberg, C. C. (1985). On the feasibility of cultural interventions in organizations. In P. J. Frost, L. F. Moore, M. R. Loius, C. C. Lundberg, \& J. Martin (Eds.), Organizational culture (pp. 169-186). Newberry Park. CA: Sage.

Makino, S., \& Inkpen, A. (2003). Knowledge seeking FDI and learning across borders. In M. Easterby-Smith \& M. Lyles (Eds.), Handbook of organizational learning and knowledge management (pp. 233-252). Malden, MA: Blackwell. 
Malone, T. W. (1987). Modeling coordination in organizations and markets. Management Science, 33, 1317-1332.

March, J. G., \& Simon, H. A. (1958). Organizations. New York: Wiley.

Marks, M. Lee, \& Mirvis, P. H. (2001). Making mergers and acquisitions work: Strategic and psychological preparation. Academy of Management Executive, 15(2), 80-92.

Martin-Cruz, N., Martin-Perez, V., \& Trevilla-Cantero, C. (2009). The influence of employee motivation on knowledge transfer. Journal of Knowledge Management, 13, 478-490.

Martin-Perez, V., Martin-Cruz, N., \& Estrada-Vaquero, I. (2012). The influence of organizational design on knowledge transfer. Journal of Knowledge Management, 16, 418-434.

Maznevski, M. L., \& Chudoba, K. M. (2000). Bridging space over time: Global virtual team dynamics and effectiveness. Organization Science, 11, 473-492.

Mesmer-Magnus, J. R., \& DeChurch, L. A. (2009). Information sharing and team performance: A meta-analysis. Journal of Applied Psychology, 94, 535-546.

Miller, V. D., \& Jablin, F. M. (1991). Information seeking during organizational entry: Influences, tactics, and a model of the process. Academy of Management Review, $16(1), 92-120$.

Miller, V. D., Johnson, J. R., \& Grau, J. (1994). Antecedents to willingness to participate in a planned organizational change. Journal of Applied Communication Research, 22, 5980 .

Minbaeva, D. (2005). HRM practices and MNC knowledge transfer. Personnel Review, 34, $125-144$.

Minbaeva, D., Pedersen, T., Bjorkman, I., Fey, C., \& Park, H. J. (2003). MNC knowledge transfer, subsidiary absorptive capacity and HRM. Journal of International Business Studies, 34, 586-99.

Miranda, S. M., \& Saunders, C. S. (2003). The social construction of meaning: An alternative perspective on information sharing. Information Systems Research, 14, 87-106.

Moon, M. J. (2000). Organizational commitment revisited in new public management: Motivation, organizational culture, sector, and managerial level. Public Performance \& Management Review, 24(2), 177-194.

Morishima, M. (1991). Information sharing and firm performance in Japan. Industrial Relations: A Journal of Economy and Society, 30(1), 37-61.

Morrison, E. W. (1993). Longitudinal study of the effects of information seeking on newcomer socialization. Journal of Applied Psychology, 78, 173-183.

Morrison, E. W. (2002). Information seeking within organizations. Human Communication Research, 28(2), 229-242. 
Morrison, E. W., \& Vancouver, J. B. (2000). Within-person analysis of information seeking: The effects of perceived costs and benefits. Journal of Management, 26(1), 119-137.

Mottola, G. R., Bachman, B. A., Gaertner, S. L., \& Dovidio, J. F. (1997). How groups merge: The effects of merger integration patterns on anticipated commitment to the merged organization. Journal of Applied Social Psychology, 27, 1335-1358.

Mowery, D. C., Oxley, J. E. and Silverman, B. S. (1996). Strategic alliances and interfirm knowledge transfer. Strategic Management Journal, 17 (Winter), 77-91.

Mujtaba, B. G., \& Alsua, C. J. (2011). Task and relationship orientation of Americans: a study of gender, age, and work experience. Journal of Behavioral Studies in Business, 3, 27 36.

Munro, D., Schumaker, J. F., \& Carr, S. C. (1997). Motivation and culture. New York: Routledge.

Murdock, G. P., \& Provost, C. (1973). Measurement of cultural complexity. Ethnology , 12, 379-392.

Nadler, A., Ellis, S., \& Bar, I.. (2003). To seek or not to seek: The relationship between help seeking and job performance evaluations as moderated by task-relevant expertise. Journal of Applied Social Psychology, 33, 91-109.

Nahapiet, J., \& Ghoshal, S. (1998). Social capital, intellectual capital, and the organizational advantage. Academy of Management Review, 23, 242-266.

Narayanan, S., Balasubramanian, S., \& Swaminathan, J. M. (2009). A Matter of balance: Specialization, task variety, and individual learning in a software maintenance environment. Management Science, 55, 1861-1876.

Naylor, J. C., \& Dickson, T. L. (1969). Task structure, work structure, and team performance. Journal of Applied Psychology, 53, 167-177.

O’Dell, C., \& Grayson, C. J. (1998). If only we knew what we know: Identification and transfer of internal best practices. California Management Review, 40, 154-174.

Ocker, R. J., \& Fjermestad, J. (2000). High versus low performing virtual design teams: A preliminary analysis of communication. Proceedings of the 33rd Hawaii International Conference on System Sciences, IEEE.

Olie, R. (1994). Shades of culture and institutions-in international mergers. Organization Studies, 15, 381-405.

O'Reilly, C. (1989). Corporations, culture, and commitment: Motivation and social control in organizations. California Management Review, 31(4), 9-25.

Osterloh, M., \& Frey, B. S. (2000). Motivation, knowledge transfer, and organizational forms. Organization Science, 11, 538-550.

Osterloh, M., Frost, J., \& Frey, B. S. (2002). Dynamics of motivation in new organizational forms. International Journal of the Economics of Business, 9, 61-77. 
Peltoniemi, M. (2007). Why do managers from different firms exchange information? A case study from a knowledge intensive industry. The Electronic Journal of Knowledge Management, 5, 81 -88.

Phelan, S. E. (2004). Using agent-based simulation to examine the robustness of up-or-out promotion systems in universities. Nonlinear Dynamics, Psychology, and Life Sciences, 8, 177-203.

Phelan, S. E. (2004). Using agent-based simulation to examine the robustness of up-or-out promotion systems in universities. Nonlinear Dynamics, Psychology, and Life Sciences, 8, 177-203.

Phillips, K. W., Mannix, E., A., Nealec, M. A., \& Gruenfeld, D. H. (2004). Diverse groups and information sharing: The effects of congruent ties. Journal of Experimental Social Psychology, 40, 497-510.

Porter, M. E. (1987). From competitive advantage to corporate strategy. Harvard Business Review, 65(3), 43-59.

Prietula, M. J., \& Carley, K. M. (1994). Computational organization theory: Autonomous agents and emergent behavior. Journal of Organizational Computing \& Electronic Commerce. 4, 41-83.

Pullar, G. L. (1992). Ethnic identity, cultural pride, and generations of baggage: A personal experience. Arctic Anthropology 29(2), 182-191.

Quigley, N. R., Tesluk, P. E., Locke, E. A., \& Bartol, K. M. (2007). A multilevel investigation of the motivational mechanisms underlying knowledge sharing and performance. Organization Science, 18, 71-88.

Raban, D. R., \& Rafaeli, S. (2007). Investigating ownership and the willingness to share information online. Computers in Human Behavior, 23, 2367-2382.

Reagans, R., \& McEvily, B. (2003). Network structure and knowledge transfer: The effects of cohesion and range. Administrative Science Quarterly, 48, 240-267.

Ren, Y., Carley, K. M., \& Argote, L. (2006). The contingent effects of transactive memory: When is it more beneficial to know what others know? Management Science, 52(5), 671-682.

Reyes-Garcia, V., Byron, E., Vadez, V., Godoy, R., Apaza, L., Limache, E. P., Leaonard, W. R., \& Wilke, D. (2003). Measuring culture as shared knowledge: Do data collection formats matter? Field Methods, 15, 1-22.

Rice, R. E., \& Aydin, C. (1991). Attitudes toward new organizational technology: Network proximity as a mechanism for social information processing. Administrative Science Quarterly, 36(2), 219-244.

Robinson, S. (1997). Simulation model verification and validation: Increasing the users' confidence. In S. Andradcttir, K. J. Healy, D. H. Withers, and B. L. Nelson (Eds.), Proceeding of the 1997 Winter Simulation Conference, The Society for Computer Simulation, San Diego, CA, (pp. 53-59). 
Romney, A. K. (1999). Cultural consensus as a statistical model. Current Anthropology, 40, S103-S115.

Rowe, F., \& Struck, D. (1999). Cultural values, media richness and telecommunication use in an organization. Accounting, Management and Information Technologies, 9, 161-192.

Rulke, D. L. \& Galaskiewicz, J. (2000). Distribution of knowledge, group network structure, and group performance. Management Science, 46, 612-625.

Sackmann, S. A. (1997). Cultural complexity in organizations: Inherent contrasts and contradictions. New York: Sage Publications, Inc.

Salancik, G. R. \& Pfeffer, J. (1978). A social information professing approach to job attitudes and task design. Administrative Science Quarterly, 23, 224-253.

Sanchez, J. C., Lanero, A., Yurrebaso, A., \& Tejero, B. (2007). Cultura y desfases culturales de los equipos de trabajo: Implicaciones para el compromiso organizacional [In Spanish: Culture and cultural gaps in work teams: Implications for organisational commitment]. Psicothema, 19, 218-224.

Schein, E. H. (1990). Organizational culture. American Psychologist, 45, 109-119.

Schlegelmilch, B. B. \& Chini, T. C. (2003). Knowledge transfer between marketing functions in multinational companies: A conceptual model. International Business Review, 12, $215-232$.

Schreiber, C., \& Carley, K. M. (2004a). Construct - A multi-agent network model for the coevolution of agents and socio-cultural environments. Carnegie Mellon University, School of Computer Science, Institute for Software Research International, Technical Report CMU-ISRI-04-109.

Schreiber, C., \& Carley. K. M. (2004b). Going beyond the data: Empirical validation leading to grounded theory. Computational and Mathematical Organization Theory, 10(2):155-164.

Schreiber, C., \& Carley, K. M. (2007). Agent interactions in Construct: An empirical validation using calibrated grounding. 2007 BRIMS Conference Proceedings, Norfolk, VA.

Schweiger, D. M., \& Walsh, J. P. (1990). Mergers and acquisitions: An interdisciplinary view. Research in Personnel and Human Resources Management, 8, 41-107.

Shaw, M. E. (1981). Group dynamics: The psychology of small group behavior. New York: McGraw-Hill.

Sherif, M., Harvey, O.J., White, B.J., Hood, W., \& Sherif, C.W. (1961). Intergroup conflict and cooperation: The Robbers Cave experiment. Norman, OK: The University Book Exchange.

Shrivastava, P. (1986). Postmerger integration. Journal of Business Strategy, 7, 65-76. 
Shoham, Y. \& Tennenholtz, M. (1994). Co-learning and the evolution of social activity (STAN-CS-TR-94-1511). Stanford, CA: Stanford University, Dept. of Computer Science.

Simon, H. A. (1945). Administrative behavior: A study of decision-making processes in administrative organization. New York: Macmillan Company.

Simon, H. A. (1957). Models of man: Social and rational; Mathematical essays on rational human behavior in a social setting. New York: John Wiley.

Smith, K. G., Carroll, S. J., \& Ashford, S. J. (1995). Intra-and interorganizational cooperation: Toward a research agenda. Academy of Management Journal, 38(1), 7-23.

Stasser, G., Titus, W. (1985). Pooling of unshared information in group decision making: Biased information sampling during discussion. Journal of Personality and Social Psychology, 48, 1467-1478.

Stasser, G., Titus, W. (1987). Effects of information load and percentage of shared information on the dissemination of unshared information during group discussion. Journal of Personality and Social Psychology, 53, 81-93.

Stasser, G., Vaughan, S. I., \& Stewart, D. D. (2000). Pooling unshared information: The benefits of knowing how access to information is distributed among group members. Organizational Behavior and Human Decision Processes, 82, 102-116.

Stasser, G. \& Stewart, D. (1992). Discovery of hidden profiles by decision making groups: Solving a problem versus making a judgment. Journal of Personality and Social Psychology, 63, 426-434.

Stasser, G., Taylor, L. A., \& Hanna, C. (1989). Information sampling in structured and unstructured discussions of three- and six-person groups. Journal of Personality and Social Psychology, 57, 67-78.

Steiner, I. D. (1972). Group process and productivity. New York: Academic Press.

Stryker, S. (1980). Symbolic interactionism: A Social structural version. Menlo Park, CA: Benjamin/Cummings Publishing.

Sydow, J., \& Windeler, A. (1998). Organizing and evaluating interfirm networks: A structurationist perspective on network processes and effectiveness. Organization Science, 9, 265-284.

Szulanski, G. (1996). Exploring internal stickiness: Impediments to the transfer of best practice within the firm. Strategic Management Journal, 17, 27-43.

Szulanski, G. (2000). The process of knowledge transfer: A diachronic analysis of stickiness. Organizational Behavior and Human Decision Processes, 82, 9-27.

Tajfel, H., \& Turner, J. C. (1979). An integrative theory of intergroup conflict. In W. G. Austin and S. Worchel (Eds.), The social psychology of intergroup relations. Monterey, CA: Brooks/Cole Publishing. 
Tajfel, H., \& Turner, J. C. (1986). The social identity theory of intergroup behavior. In S. Worchel \& W. G. Austin (Eds.), Psychology of intergroup relations (2nd ed., pp. 724). Chicago: Nelson-Hall.

Tushman, M. L., \& Nadler, D. A. (1978). Information processing as an integrating concept in organizational design. Academy of Management Review, 3(3), 613-624.

Ullrich, J., Wieseke, J., \& Van Dick, R. (2005). Continuity and change in mergers and acquisitions: A social identity case study of a German industrial merger. Journal of Management Studies, 42, 1549-1569.

Uzzi, B. (1997). Social structure and competition in interfirm networks: The paradox of embeddedness. Administrative Science Quarterly, 42, 35-67.

Vaara, E. (2002). On the discursive construction of success/failure in narratives of postmerger integration. Organization studies, 23(2), 211-248.

Vaighn, D. (1996). The Challenger launch decision: Risky technology culture and deviance at $N A S A$. Chicago: University of Chicago Press.

Van Der Vegt, G. S., Emans, B. J., \& Van De Vliert, E. (1998). Motivating effects of task and outcome interdependence in work teams. Group \& Organization Management, 23(2), 124-143.

Van Der Vegt, G. S., Emans, B. J., \& Van De Vliert, E. (2001). Patterns of interdependence in work teams: A two-level investigation of the relations with job and team satisfaction. Personnel Psychology, 54(1), 51-69.

Van Der Vegt, G. S., Van De Vliert, E., \& Oosterhof, A. (2003). Informational dissimilarity and organizational citizenship behavior: The role of intrateam interdependence and team identification. Academy of Management Journal, 46(6), 715-727.

Van de Ven, A. H. \& Delbecq, A. L. (1974). A task contingent model of work-unit structure. Administrative Science Quarterly, 19, 183-197.

Van de Ven, A. H., Delbecq, A. L., \& Koenig, Jr., R. (1976). Determinants of coordination modes within organizations. American Sociological Review, 41, 322-338.

van Knippenberg D., van Knippenberg B., Monden L., \& de Lima, F. (2002). Organizational identification after a merger: A social identity perspective. British Journal of Social Psychology 41, 233-252.

van Leeuwen, E., van Knippenberg, D., \& Ellemers, N. (2003). Continuing and changing group identities: The effects of merging on social identification and ingroup bias. Personality and Social Psychology Bulletin, 29, 679-690.

van Maanen, J., \& Barley, S. R. (1985). Cultural organization: Fragments of a theory. In P.J. Frost, L. F. Moore, C. C. Lundberg, \& J. Martin (Eds.), Organizational culture (pp. 31-54). Newberry Park, CA: Sage. 
van Wijk, R., Jansen, J. J. P., \& Lyles, M. A. (2008). Inter- and intra-organizational knowledge transfer: A meta-analytic review and assessment of its antecedents and consequences. Journal of Management Studies, 45, 830-853.

van Wijk, R., van den Bosch, F. A. J., Volberda, H. W., \& Heinhuis, S. M. (2005). Reciprocity of knowledge flows in internal network forms of organizing. ERIM Report Series Research in Management (ERS-2005-024-STR), Rotterdam School of Management, Erasmus University.

Van Zandt, T. (1997). Decentralized information processing in the theory of organizations. In Murat Sertel (Ed.), Economic design and behavior, (pgs. 7-125). London: MacMillan Press Ltd.

Van Zandt, Ti. (1999). Real-time decentralized information processing as a model of organizations with boundedly rational agents. Review of Economic Studies, 66, 633658.

Vestring, T., King, B., \& Rouse, T. (2003). Should you always merge cultures? Harvard Management Update, May, p. 10.

Viswanath, K., Kahn, E., Finnegan, Jr., J. R., Hertog, J., \& Potter, J. D. (1993). Motivation and the knowledge gap. Communication Research, 20, 546-563.

Von Krogh, G. (2003). Knowledge sharing and the communal resource. In M. Easterby-Smith \& M. Lyles (Eds.), Handbook of organizational learning and knowledge management (pp. 372-392). Malden, MA: Blackwell.

Weber, R. A., \& Camerer, C. F. (2003). Cultural conflict and merger failure: An experimental approach. Management Science, 49, 400-415.

Wegner, D. M., Giuliano, T., \& Hertel, P. T. (1985). Cognitive interdependence in close relationships. In W. J. Ickes (Ed.), Compatible and incompatible relationships (pp. 253-276). New York: Springer.

Weiler, A. (2005). Information-seeking behavior in generation Y students: motivation, critical thinking, and learning theory. The Journal of Academic Librarianship, 31, 46-53.

Wernerfelt, B. (1984). A resource-based view of the firm. Strategic Management Journal, $5(2), 171-180$.

Wickesberg, A. K. (1968). Communications networks in the business organization structure. Academy of Management Journal, 11(3), 253-262.

Williamson, O. E. (1971). The vertical integration of production: market failure considerations. The American Economic Review, 61(2), 112-123.

Winter, S. G. (1987). Knowledge and competence as strategic assets. Cambridge: Ballinger.

Wong, S-S., \& Burton, R. M. (2000). Virtual teams: What are their characteristics and impact on team performance? Computational \& Mathematical Organization Theory, 6, 339360. 
Wood, R. E. (1986). Task complexity: Definition of the construct. Organizational Behavior and Human Decision Processes, 37, 60-82.

Wood, R., \& Bandura, A. (1989). Social cognitive theory of organizational management. Academy of Management Review, 14, 361-384.

Yilmaz, C., \& Hunt, S. D. (2001). Salesperson cooperation: The influence of relational, task, organizational, and personal factors. Journal of the Academy of Marketing Science, $29(4), 335-357$.

Zander, U., \& Kogut, B. (1995). Knowledge and the speed of the transfer and imitation of organizational capabilities: An empirical test. Organization Science, 6, 76-92. 


\section{APPENDIX A: SYNOPSIS OF COMPUTATIONAL MODELS}

This study employs an agent-based, computational model to simulate the post-merger integration process. This section provides a synopsis of the basic models used and describes the script of the virtual merger, including the synthesized scenarios to which the computer simulation is guided and from which the virtual experiments are designed. The core features of the model include the virtual merger script, the computational representation of the agents and their environment, the agent behavioral model, and the merger transaction. These features are described below.

\section{A.1 Computational Model}

This research is conducted in a computational laboratory (Burton, 2003); where computer simulations are to create data for the statistical modeling and analysis that provide support to the research. The organizational computational model used for all experiments in this study is the Construct model of social behavior as implemented in Construct, which is software developed and maintained by CASOS. Agent based models, and Construct in particular, consists of two interrelated aspects: the representation model, and the behavioral model. Moreover, in this special case of a merger, an additional third aspect is necessary to define: the notion of the merger event. The three aspects are described below.

\section{A.2 Virtual Merger Script}

For each experiment, the same script takes place within the computer simulation laboratory: two independent organizations, Alpha and Beta, are thrust into a merger to form NewCo. Alpha and Beta provide similar products and services that complete in the same market and thus it is a horizontal merger. Alpha (the acquirer) is seeking to gain a larger share of its market and hopes to gain economies of scale through various synergies by combining with Beta. Cost-cutting is not part of NewCo's forward strategy; moreover, workforce redundancies are not of concern and therefore layoffs not part of the virtual script.

Since they compete in the same industry, there are many similarities between Alpha and Beta. Both are demographically stable organizations and each has matured into a coherent 
and industrious group of individual actors working with a purpose towards maximizing their organization's performance. There exists a tight intra-team social network among the actors with inter-team ties forming a united organization. As is the case in the real-world, there are also a few random social-ties between members of Alpha and Beta. Both organizations are organized by work teams that, through the efforts of individuals within the team, aim to accomplish a specific set of virtual tasks. The individual actors and their super-ordinate teams master their assigned tasks by accumulating local tacit knowledge that is diffused to all members of the same team in due course. Moreover, the two organizations also have a specific virtual culture that can be supposed as global knowledge and is transmitted to members of the same organization.

However, though they navigate in the same environment, because of their unique histories there is the prospect of numerous differences--or possibly further similarities-between Alpha and Beta, e.g. the number of employees, the number of work teams, the level of teamwork within the teams, the level of technological support, the norms and paradigms embedded in their work culture, et cetera. These pre-merger characteristics formulate the explicitly designed scenarios that will be implemented as the virtual experiments' control variables, which are later described in detail and substantiated in depth.

At the start of the virtual experiment, when Alpha and Beta are actually merged, the components of each are commingled at the team-level task under the NewCo umbrella. Consequently NewCo consists entirely of the pre-merger characteristics and pre-existing social networks of Alpha and Beta. This situates NewCo as a possibly eclectic mixture of socio-technical sub-networks that have become intermingled and must now interact according to the proscribed social behavior theories contained in the computational model, i.e., Construct.

\section{A.3 Representation Model}

The representation model implemented within Construct is based on the meta-network construct (Carley, 2002a, 2002b), which is a primary feature of Dynamic Network Analysis (Carley, 2003). Numerous networks are formed from three basic entity types: actors, 
knowledge and tasks. Broadly, the representation model is consistent with the PCANS model (Krackhardt \& Carley, 1998). Table A-1 lists these entity types and provides a summary of the type of networks that form the basis of the representation model. From the three entity types, four types of networks are utilized. The first is the social network, which is an actor-toactor relationship. The second is an assignment network consisting of actor-to-task relationships. The third is a task-to-knowledge network which represents the concepts that are necessary to perform each task. The fourth is the knowledge network which is an agent-totask network representing what concepts each agent knows.

Table A-1. Organizational Representation: Ontologically coded socio-technical networks.

Entity Types

\begin{tabular}{l|c|c|c}
\cline { 3 - 4 } \multicolumn{2}{c}{ Entity Type } & \multicolumn{1}{c}{ Actor } & \multicolumn{2}{c}{ Knowledge } & Tasks \\
\hline \multirow{2}{*}{ Actor } & Social Network & $\begin{array}{c}\text { Knowledge } \\
\text { Network }\end{array}$ & Assignment Network \\
\cline { 2 - 4 } & & & $\begin{array}{c}\text { Knowledge Requirement } \\
\text { Knowledge }\end{array}$ \\
\cline { 2 - 4 } Task & & & - \\
\hline
\end{tabular}

\section{A.4 Agent Behavioral Model}

The behavioral model that controls how agents operate in the simulations is an operationalization of the Construct model (Carley, 1991). Construct theory, or constructuralism, has its underpinnings in social theories such as symbolic interactionism (Blumer, 1969), structural interactionism (Stryker, 1980), and structural differentiation theory (Blau, 1970). In the Construct model, the information-processing perspective of the Carnegie School (Simon, 1957; March \& Simon, 1958; Cyert \& March, 1963), i.e., people are information seeking, is integrated with social-information-processing theory (Salancik \& Pfeffer, 1978; Rice \& Aydin, 1991), i.e., what knowledge a person has is dependent on what information they have access to via their social network. In Construct, agents purposefully 
interact through complex discourse (Dooley, Corman, McPhee, \& Kuhn, 2003) and social interaction (Carley, 1986) to exchange knowledge and frequently seek out others who have knowledge that they do not yet hold, and are sought out by others seeking their knowledge. Moreover, Construct incorporates the notion of Transactive Memory (Wegner, Giuliano, \& Hertel, 1985) into the processing model. This interaction dynamic is played out numerous times within an organization. When coupled with the organizational membership changes, e.g., hiring and firing, the emerging micro-interaction dynamics result in complex dynamics that have been validated by comparing to real-world social dynamics.

\section{A.5 Merger Transaction}

The organizational merger transaction is a single point-in-time event that involves combining the various socio-technical networks of Alpha and Beta to form NewCo. The respective organizational teams from Alpha and Beta are paired and combined at the start of the simulation run. Once NewCo is setup, as a facet of the simulation initialization process, the behavioral model described above is in full authority of the behaviors occurring within the simulation. This simulation does not differentiate any behaviors between running in an ordinary or a post-merger situation. 


\section{APPENDIX B: FIRST PERSPECTIVES OF THE ORGANIZATIONAL MODELS}

A characteristic feature of computational modeling and virtual experimentation that employs the agent-based paradigm is that the modeler has design control both over the behaviors of the virtual actors, as well as, over the environment in which the actors behave. These characteristics permit the modeler to design a comprehensive, yet simplified, virtual world, albeit once which is a highly-biased subset of reality, constructed entirely according to the modeler's wishes. Correctly understanding the outcomes of virtual world experiments requires one to be fully-informed about central behaviors embodied in the virtual world. There are three fundamental, design perspectives employed in the virtual world designed for this research: (a) I model an organization as a bounded assembly of actors preoccupied with searching for, collecting, and exchanging information with other actors, i.e., an a group of myopic, information-processing actors (b) I embrace the standpoint that organizational culture fundamentally consists of shared knowledge among the members of a group, and (c) I hold that task performance is directly correlated with holding the knowledge necessary to perform that task, performance is measures by the degree to which an actor holds the knowledge specifically required to perform that task. These three perspectives are expounded in more detail below.

\section{B.1 Organization as Information Processing Actors}

I embrace the information processing view of an organization: An organization is an organized collection of agents, each of which is a distinct information processor. The Carnegie School of Organizational Theory (Cyert \& March, 1963; March \& Simon, 1958; Simon, 1957) proposed this information-processing perspective as a basis for organizational decision making. According to this information centric perspective, individual behavior and ultimately organizational performance can be explained in terms of what information is available to an organization and specifically who within the organization holds the information; this critical for performing tasks effectively. In an organization, actors interact as 
a matter of routine, and in the course of their interaction, they exchange such information. This is to say that they exchange knowledge amongst one another as they interact, either through direct person-to-person communications or through indirect forms such as through electronic and broad-cast media (Dooley \& Corman, 2004). The transfer of this knowledge through-out the members of an organization is critical from social and organizational perspectives, and underpins concepts such as organizational learning (Argote, Gruenfeld, \& Naquin, 2000).

Conceptually, an organization is viewed as social construct designed for an instrument fashioned to handling information; abstractly, a machine a machine, perhaps. Within its boundaries, an organization is essentially an information processing system with complex internal dynamics. Subscribing to the Carnegie School of Organizational Theory (Cyert \& March, 1963; March \& Simon, 1958; Simon, 1957), one considers that the organizations information-processing capability is a critical underpinning for decision making and performance. There have been many attempts at precisely defining the term information without settlement on any concrete consensus (Case, 2007); Herein, since the real-world is only being abstractly modeled, the meaning of the term is preserved as remaining highlyabstract. For example, Chaffee's (1991, p. 9) suggestion that information is "anything that exists psychologically for a person" as a possible guidepost to how to idealize information within the confines of this study. Also note that often the term knowledge is used in the contextual discussion of this paper; readers should make no distinction between the two terms and should remain rather flexible and become unattached in envisioning the boundaries of this central construct.

\section{B.2 Information Exchange}

As social beings, organizational actors interact with one another as a matter of natural routine, .i.e., they socialize. In the course of this never-ending socialization process, actors exchange information with one another, intentionally or unintentionally, with or without purpose. They pass information either through direct person-to-person communications or through more indirect and contemporary forms, such as electronic emails and broadcast media 
(Dooley \& Corman, 2004). Keeping with our abstract understanding for information, the process by which information is exchanged with others, e.g., verbally, visually, even subconsciously, should also be held as being highly abstract.

The organizational processes supportive of information exchange, or knowledge transfer, are "a basis for competitive advantage" (Argote \& Ingram, 2000, p. 150; Zander \& Kogut, 1995). Consequently, information exchange is a predominant concern of management during post-merger integration, since mergers are primarily consummated for the purpose of improving a firm's competitive advantage (Bruner, 2004). The exchange of information among the members of an organization is critical to individual and organizational performance from both social and actionable perspectives, and supports central concepts such as organizational learning (Argote, Gruenfeld, \& Naquin, 2000). In the usual sense, knowledge transfer refers to the transferal of existing "reservoirs of knowledge" (Argote \& Ingram, 2000, p. 153) across organizationally-bounded groups; however, this convention is amended to refer to such transfer occurring "within a newly-combined group" rather than across. Furthermore, pre-merger groups will hold unique reservoirs of knowledge such that the a subset of the constituent elements--e.g., the tactic and extrinsic skills, contextual practices, routines, etc--, are particular and specific to their group; this holds nonetheless, even if two groups perform the same functional tasks; every organization has unique differences in its work processes, procedures, etc.

In the special setting brought about during post-merger integration, effective knowledge transfer is paramount. In order to realize the newly expected post-merger performance level, the previous disjoint knowledge reservoirs must ultimately meld into a single pool of shared knowledge - a post-merger knowledge reservoir united within a single group. Note that as part of the pre-merger planning, finances can easily be combined in an orderly and predictive matter; however, in the realm of an organization's knowledge-base, this integration of human knowledge is more of a complex and dynamic --perhaps chaoticprocess (Szulanski, 2000), as opposed to the obviously-important but comparatively lessdimensional legal and accounting transactional events. 


\section{B.3 Factors affecting the Transfer of Knowledge}

Operative organizational knowledge transfer is a hard to accomplish (Argote, 1999; Szulanski, 2000) and is influenced by numerous factors (Becker \& Knudsen, 2006) and subprocesses, e.g., practices such as actor rotation between groups can facilitate transfer under the right conditions (Almeida \& Kogut, 1999; Argote, 1999). In the literature, there is keen awareness and conceptual models of this intra-firm transfer process, e.g., Schlegelmilch and Chini (2003). Knowledge transfer in an organization is a process with specific constituent stages with different factors affecting its stickiness (Szulanski, 1996, 2000). The communication among a work team is critical to the development of new ideas and knowledge (Boland \& Tenkasi, 1995). In a laboratory setting, it was established that social identity affects the transfer of knowledge (Kane, Argote, \& Levine, 2005): when an individual socially-identifies with a group (and the group likewise identifies with the individual), as social identity theory (Tajfel \& Turner, 1979, 1986) suggest as being a factor in intergroup behavior, it has been found that the individuals view of the group (and the groups view of the individual) is positive. Therefore, as derived in Kane, Argote, and Levine "individuals may feel more comfortable sharing knowledge with groups with whom then share social identity than with those that they do not.."'(2005, p.57). It has been shown that an individual whom has temporarily left their social group to visit another group and obtain outside knowledge and ideas, returns to find that they have less influence in their home group (Gruenfeld, Martorana, \& Fan, 2000) and that such integration is essential to knowledge flow (van Wijk, Jansen, \& Lyles, 2008; van Wijk, van den Bosch, Volberda, \& Heinhuis, 2005). Research has also found that there can be inequalities among social groups in the availability and acquisition of information (Viswanath, Kahn, Finnegan, Jr., Hertog, \& Potter, 1993).

\section{B.4 Organizational Knowledge}

In this study, attention is limited to knowledge that is solely relevant to the performance of a specific task, though other information can be vitally important, e.g. ideas to advance group innovation (Inkpen \& Dinur, 1998). These task relevant concepts are referred to as task-knowledge. Like many other aspects of this model, exactly what constitutes a relevant concept of knowledge is highly abstract although it steadfastly is some specific 
information that is necessary to perform the given task expertly. For example, in a pizzamaking task, knowledge of how to toss the dough is relevant to making pizza as is knowledge of how to combine the ingredients making the pizza sauce; while, knowing how to clean an over may be useful to a pizza maker, it is not necessarily essential to being a world-class pizza maker (cleaning the oven might be abstracted as being a separate task, perhaps).

\section{B.5 Uniqueness of Organizational Knowledge}

This study focuses on the knowledge transfer in the combined, post-merger organization. In particular, the focus is on the task-specific knowledge that is unique to previously detached workgroups and that has become essential to newly formed groups, such that the knowledge is essential for workers to complete a given task. In some work teams, knowing how to access information contained in the group is essential (Stasser, Vaughan, \& Stewart, 2000). Research indicates that organization change can affect knowledge transfer, and thus performance (Lin, 2000). Since each organization has specific knowledge unique to itself, for the post-merger organization to fully integrate, this knowledge must be diffused to others in the combined entity. This study intendeds to be a step toward developing a predictive capability for real-world mergers, thus it incorporates the pre-merger characteristics of the two organizations into the study design. The study aim to uncover the correlations--not necessarily causality--between the performance-relevant knowledge transfer rate in the combined organization with that of relatively easy to estimate characteristics of the two organizations.

Furthermore, the experiment is restricted to the set of task-knowledge concepts that is

mutually exclusive across the same task in Alpha and Beta. For example, in a pizza-making task, knowledge of how to toss the dough is common knowledge across two organizations and is therefore not captured in this virtual environment. However, knowledge of how to combine the special ingredients making the pizza sauce differs across organizations and is therefore deemed as a task knowledge concept in this virtual environment. Only organizational unique concepts are modeled herein; it would be meaningless to track the flow of information 
throughout an organization if, in fact, those that need to know a particular informational concept, already do.

\section{B.6 Shared Knowledge is Shared Culture}

Organizational culture can be learned shared knowledge in a group (Reyes-Garcia et al., 2003). This cultural knowledge is preserved through a socialization process (Schein, 1990) that involves transferring cultural information verbally, through behavioral cues, artifacts, etc. In this study, an anthropological and cognitive views of culture (Duranti, 1997) are taken, which regards culture as the sum total of a group's knowledge (Linton, 1940) consisting of the facts of the cultural norms, traditions, expectations, customary behaviors, etc. A contemporary example of cultural knowledge such as a norm in the organization setting is when and in what circumstances it is acceptable to use email instead of using direct verbal communication (El-Shinnawy \& Markus, 1998; Rowe \& Struck, 1999), or even submit to using social media. This knowledge of one's culture is held by each member of a group or organization in the form of specific knowledge concepts (D'Andrade, 1995; Kroeber \& Kluckholm, 1952; Romney, 1999). The members of a cultural group intimately know detailed aspects about their culture which are implicitly and explicitly shared and reinforced with other in-group persons. Often such knowledge is held tightly within the group without transparency and can sometimes intentionally be with-held from out-group persons in order to exclude unwanted others from the group. Herein the perspective that a specific culture itself is a pool of shared and distinctive cultural information that is uniquely held by members of a specific group (D'Andrade, 1981), and that such information is not necessarily known or embraced by others outside of that particular group (Cremer, 1993). In practice, however, it may not be in the strategic interest that the combined organization meld the two antecedent cultures into one (Vestring, King, \& Rouse, 2003).

\section{B.7 Task Knowledge is Task Performance}

Naylor and Dickson (1969) offer that a factor of team performance is the structure of the task itself and that the complexity of the task is then based on the amount of information required to perform that task. A similar perspective is adopted here: task performance is 
directly associated with task knowledge, which is also aligned with other research (Beirly \& Chakrabarti, 1996; DeCarolis \& Dees, 1999). It follows, all other aspects being equal that the more task-specific knowledge an actor has for performing a given task, the better the actor will be at performing that task, and greater the amount of knowledge necessary for performing a given task, the higher the degree of difficulty will be for performing the task. Tasks can differ in the level of their complexity (Wood, 1986): that is, the number of knowledge concepts assigned to a specific task according to the complexity of that task, implies that a more complex task takes longer to master. An illustrative example of this would be a neighborhood pizza shop: If a pizza maker has no knowledge of how to make pizza, it would clearly result in a disappointing meal, while a pizza-maker with expert knowledge of how to make pizza, you can reasonably be assured you will have a delicious pizza nearly every time. This task knowledge often can be transferred from person to person and from group to group (Darr et al., 1995). The influence of localized culture on task performance is acknowledged, but for this study such task-performance motivation is removed from consideration in order to focus on the wider, more global cultural dimension of inclination for information exchange.

In a team setting, members of a group each bring different expertise, knowledge, and information into their group (Jackson, 1992) and the distribution of the knowledge within a group varies (Liang, 1994). The knowledge held by a group directly influences the performance of the group (Shaw 1981; Steiner 1972; Stasser \& Stewart, 1992). The distribution of the knowledge within the group can be deliberately managed (King, 1999) and greatly affects group performance (Rulke \& Galaskiewicz, 2000). How broadly the information is distributed and the amount of information shared within the group has been shown to affect the group performance (Stasser, Taylor, \& Hanna, 1989; Stasser \& Titus, 1985, 1987). Moreover, there can be complicated social dynamics occurring within a small group when it comes to exchanging such information (Deeker, Stokman, \& Franses, 2000). Localized knowledge common to a specific group can face natural difficulties being transferred outside of that group (Almeida \& Kogut, 1999), unless there is exchange of personnel among the group, such as within an industry, for example; in our case herein, melding human assets within a newly combined organization. 
To evaluate the progress of the organization during the post-merger integration period, thus measuring performance, task-knowledge capabilities within the merged organization are focused on. The amount of knowledge that has been transferred not from a generic perspective, but from the qualitative perspective is measured. That is to say, the knowledge transfer for only those concepts that have value to the specific members of the organization, necessary to perform their work tasks, given the task-assignment structure of individual work units, is of relevance and consequence. 


\section{APPENDIX C: CONSTRUCT THEORY}

Construct (Carley, 1990; Schreiber \& Carley, 2004a) is a computational model embodied in computer software that provides both a manifestation of an organizational theory and a platform for researchers to experiment. It is both a theory of social interaction and a description of an agent-based model (ABM) of the theory. It is based on well-established social principals and ground on other social theories; it reproduces individual human-agents interacting and operating within a task-performing organization. The purpose of the Construct $\mathrm{ABM}$ is to simulate the social dynamics of individuals within a goal-seeking organization, so that organization-level outcomes can be studied (Schreiber \& Carley, 2007). Construct reflects the concept that human-agent's behavior is influenced by external factors (other agents and their environment), and that the external factors are, in-turn, affected by the agent. Agents recurrently interact with their environment and socialize (interact) with other agents within that changing environment; subsequently, the agents integrate feedback resulting from their actions and those taken by other agents. Construct can therefore be used to model human groups, sub-groups and entire organizations, as agent-based complex systems. The interdependent, individual agent-interactions result in considerable non-linearity in organizational-level outcomes, such as collective group behaviors, the distribution of knowledge and overall organizational performance.

Construct's representation of agent-based behavior has its roots in symbolic interactionism (Blumer, 1969), structural differentiation theory (Blau, 1970) and structural interactionism (Stryker, 1980). Formally, these theories are combined into a social theory called Constructuralism (Carley, 1991) which is embodied in the Construct model the operationalization of that model, Construct software. It incorporates the concept of decentralized information processing (VanZandt, 1997, 1999). Among its many applications, for example, Construct can be used to simulate the diffusion of beliefs and ideas through groups as constrained and enabled by the social structure of the group as well as through nonhuman agents, such as communication technologies and processes. The model has been used extensively for analysis and forecasting in industry, non-profits, emergency response, higher 
education, military and government. The Construct model has been scientifically validated numerous times (Carley \& Hill, 2001; Schreiber \& Carley, 2004a, 2007) and results have been compared against empirical datasets, e.g., Kapferer's Zambia Tailor Shop data (Carley, 1990). Over the past decade, Construct model, which has been used extensively for developing social theory continues to evolve and be refined.

The agents in Construct are sculpted simply as information processors. The Carnegie School of Organizational Theory (Simon, 1957; March \& Simon, 1958; Cyert \& March, $1963)$ proposed the information-processing perspective. From this viewpoint, individuals' behaviors could be explained in terms of what information was available to whom, the individuals' cognitive limits to information processing abilities, organizational (social and cultural) limits to access to information, the quality of the information, and so forth. Simon (1945), March and Simon (1958), and Cyert and March (1963) examined the decision-making components of group action from this same view embedded in Construct. According to this information-processing perspective of organization theory (March \& Simon, 1958; Cyert \& March, 1963; Galbraith, 1973, 1977), individual behavior and organizational performance can be explained in terms of what information is available to whom, who holds specific information and what the person's cognitive limits are, which can ultimately result in complex, collective outcomes (Iwanaga \& Namatame, 2002). In a group, the agents interact as a matter of routine, and in the course of their interaction, they exchange information; that is to say that they exchange knowledge amongst one another as they interact, either through person-to-person communications or through various forms of broadcast media (Dooley \& Corman, 2004).

\section{C.1 The Organizational Meta-network Representation Model}

The representational model employed by Construct is based on a meta-network (Carley, 2002a, 2002b), which is a core feature of Dynamic Network Analysis (Carley, 2003). Several networks are formed from three basic entity types: actors, knowledge and tasks. Largely, the representation model is consistent with the PCANS model (Krackhardt \& Carley, 1998); from the three entity types, four types of networks are utilized: (a) a social network, 
which is an actor-to-actor relationship, (b) an assignment network consisting of actor-to-task relationships, (c) a task-to-knowledge network which represents the concepts that are necessary to perform each task, and (d) a knowledge network which is an agent-to-task network representing what concepts each agent knows.

\section{C.2 The Organizational Actor as Behavioral Agent}

Agents in the construct model can represent either human or other behavioral or static entities. In the context herein, the agents to represent humans within an organization are considered. Each agent can have numerous, or no, attributes affiliated with them; whereas each attribute serves as either being identification-oriented or as a relevant determinant for some aspect of the dynamic model. An agent can be considered active or effectively dormant in any time period and this status can be affected by numerous circumstances under control of the modeler. Construct agents purposefully seek out other agents to interact with over the course of the agent's life. Essentially, each time period agents are paired up into discreet dyads, which reflect the real-world phenomenon of human interaction. Of course, real-world interaction can involve collectives whereas several agents all interact together within a specific group; construct recognizes this as a broadcast scenario. For the purposes here, the interactions are limited to only the dyads.

This is, in turn, often constrained by the individual's position in the social structure. Structure influences the individual's decision making because it constrains access to information and because the decisions, attitudes, and actions of those to whom one is structurally connected have a strong influence on behavior. Therefore, the formal reporting and the informal social structure within the organization along with task-assignment, limits access to information, determines the quality of and individual's task performance. The organization's structure can be regarded as a coordination scheme whose cost and performance depends on the network of formal and informal connections within the organization (Malone, 1987; Krackhardt, 1994; Krackhardt \& Brass, 1993; Krackhardt \& Hanson, 1993). This work is argument is forwarded by social and dynamic network theorists. 


\section{C.3 The Organizational Actor as an Information Processor}

Over the course of time, at any specific point in time, as each dyad interacts, one of the agents presents some informational tidbit to the interaction partner. Construct theory grounds characteristics of the agent to the Carnegie School of Information processing. The Carnegie School of Organizational Theory (Simon, 1957; March \& Simon, 1958; Cyert \& March, 1963) puts forth the information-processing perspective, which suggests that individual and organizational decisions can be explained in terms of what information was available to whom in the process. Information-processing theorists (March \& Simon, 1958; Cyert \& March, 1963; Galbraith, 1973, 1977) and social-information-processing theorists (Salancik \& Pfeffer, 1978; Rice \& Aydin, 1991) have argued that individual, and hence organizational, decisions depend on what information individuals have.

The Information processing perspective notes that any person can be represented in terms of their socio-demographic characteristics, technology access characteristics, and information processing characteristics. These Information processing characteristics include establishing an interaction, sending receiving, storing information, and include variations in the amount, quality, and frequency of sending or receiving information, accuracy in storage, data retention strategies, forgetting, and errors rates in transmission and reception.

An agent can indeed receive information that they already hold and is therefore redundant and has little value to the construct Agent. In construct the agent either knows a fact, or does not.

\section{C.4 The Organizational Actor as an Adaptive Agent}

Agents adapt and change their actions in accord with their goals and the environment. In the Construct model this is manifested in the selection of an interaction partner and the choice of the information to exchange (if any) with the selected partner. A probabilistic heuristic to select an interaction partner is applied to each agent in each time period that the agent is active. From this algorithm, each candidate partner agent is assigned a weighted interaction score that is normalized with all the other possible partner scores and a probability 
of interaction is assigned. Randomly, according to the relative weights of these scores a single interaction partner is selected for the principle agent. Depending on the modeler's desires, there are three ways to determine these scores: (a) unbiased, (b) according to homophily theory, and (c) according to information seeking goal. The unbiased is essentially a purely random selection process where each candidate is a fixed score. The according to homophily approach will provide higher scores for agents that are more similar to the principal agent. Similarity can be determined by the sameness of agent attributes, social network, the knowledge network, task network, et cetera. The more similar an alter is the principal in these characteristics, the higher the score. The third embedded theory for selecting an interaction partner is according to the principles goal of obtaining information they do not already have and are seeking via their social network. In this case the principal has a preference for interacting with others that they believe have the information they do not have. The agent gives preference to those agents that they believe have a specific piece of information according to transactive memory theory.

It is through these mechanisms that the scores for the selection of interaction partners changes and evolves in a non-linear manner during the lifetime of an agent and all others. The agent adapts as those around also are adapting.

Organization researchers assert that organizational dynamics are due to, and emerge from, the level of adaptiveness of the agents within the organization. This process has a variety of names, including co-learning (Shoham \& Tennenholtz, 1994) and concurrent interaction (Carley, 1991). Co-learning is the process in which multiple actors concurrently try to adapt to others' behavior with the aim of reaching the group's goals (Shoham \& Tennenholtz, 1994). Concurrent interaction and the co-evolution of self and society are necessary for the emergence of social stability and consensus. Collectively, the findings from these two models indicate that emergent social phenomena (such as the emergence of social networks) and the evolutionary dynamics (patterns of knowledge diffusion) depend on the rate at which the agents age, learn, and move, constraints on access and other organizational processes. 
An adaptive agent is one that behaves on the basis of information, but that information changes over time in response to their exchanges with other agents. Thus the agent learns and may improve task-performance. It follows that the Construct model reflects that group dynamics are due to, and may even emerge from, the level of adaptiveness of the agents within the group. This process has been referred to by a variety of names, including colearning (Shoham \& Tennenholtz, 1994), synchronization, and concurrent interaction (Carley, 1991). According to Shoham and Tennenholtz, co-learning is occurs when several agents simultaneously try to adapt to one another's behavior so as to produce desirable group-level results. According to Carley, concurrent interaction and the co-evolution of self and society is necessary for the emergence of social stability and consensus. Together, the findings from these models indicate that emergent social phenomena (such as the emergence of hierarchy) and the evolutionary dynamics (patterns of change) depend on the rate at which the agents age, learn, and move, constraints on access and other group processes.

\section{C.5 The Organizational Actor Situated in the Organizational Meta- network}

The environment is an important dimension of an agent-based model; the environment as anything outside the boundary of the agent itself. Agents are situated within an organizational meta-network that is crafted according to the PCANS model introduced by Krackhardt and Carley. This consists of their social network, task-assignment network, knowledge network.

Through interaction the agent changes connections to others, to knowledge, resources, tasks, locations. It is important to link a more macro-perspective on the organization as a whole with the more micro-perspective on the individual. The meta-network paradigm provides a way of beginning to make this linkage.

The second primary component of an agent-based simulation design, beyond the agent behavior, is the environment. It is this environment in which an agent exists and navigates within. An effective construct to represent an environment, both conceptually and concretely in the software architecture, is to bring together multiple, relational networks that capture the 
elements of the environment and the agent's relationship to those entities. By representing environment as meta-network, the modeler benefit from techniques that are available in network science-based fields, e.g., social network analysis and organizational network analysis. In this section, how the network-styled constructs are applied to representing the environment in an agent-based simulation is discussed.

Organizational learning is closely tied to the sharing or diffusion of information. As demonstrated by Granovetter $(1973,1974)$, connections or ties among individuals determine what information is diffused and to whom. However, the strength of the relationships among individuals may actually inhibit information diffusion. One reason for this is that in groups where the level of shared information is high, communication may tend repeat known information (Kaufer \& Carley, 1993); the likelihood of new information diffusing along these existing ties can actually decrease as individuals within the organization work together and become more similar in what they know. Essentially, collaborative teams can easily become less innovative over time as no new members are added. The level and pattern of ties among individuals in a group influences the speed with which information spreads and to whom it spreads (Becker, 1970; Burt, 1973, 1980; Coleman, Katz, \& Menzel, 1957, 1966; Granovetter, 1973, 1974; Lin \& Burt, 1975). Advances in the area of diffusion that are particularly relevant to organizations have been made by researchers using social network techniques. This work demonstrates that how integrated the individual is into the organization influences the likelihood that this person will diffuse new information (Burt, 1973, 1980; Kaufer \& Carley, 1993).

An effective construct in which to represent the agents' environment is the meta network. The meta-network can be described simply as multiple, relational networks with node entities of various types, such as human agents, tasks, and knowledge. In the same manner of the PCANS model (Krackhardt \& Carley, 1998) this multi-network perspective provides a formalized data structure to richly represent the various entities and relations forming an environment in which an agent co-exists. The actors' social networks, their assigned tasks, their individual knowledge, and the knowledge necessary for performing a specific task, all are adequately captured in the meta-network construct. Further, real-world 
changes, such as birth and death of an agent, the reassignment of tasks, et cetera, are all simple matters to capture and represent within the meta-network representation.

\section{C.6 The Macro-scope on the Organization}

Construct is a model of organization behavior, yet thus far only the agent and its environment has been discussed. Construct rolls-up the agents into an organization in two ways. First the interaction sphere in which an agent operates can be restricted to a sub-group of other agents, conceptualizes as a team, department, silo, et cetera. Secondly, organizational-level perspective is viewed according to a performance metric based on the notion that organizational tasks are performed to the extent that the agents assigned to a given task have the knowledge necessary to perform that task. Tasks can be assigned to individual agents, or according to the model's needs, to a team of agents. In the case of individually assigned tasks, if the single agent has the knowledge necessary to perform the task, its performance (from 0-1.0) will be determined independent of another agent's knowledge. Alternatively, a task can be assigned to a team of agents, whereas the task is performed to the level that any of the agents have the knowledge, or more restrictively, only of all of the agents that the required knowledge. In an experimental study, it was found that the more communicative virtual team outperformed the less communicative (Ocker \& Fjermestad, 2000).

\section{C.7 Construct Software \& Platform}

Construct software is part of an integrated toolset (Carley, Diesner, Reminga, \& Tsvetovat, 2007) in which data can seamlessly flow from one step to the next in the analysis process. The CASOS integrated toolset enables the text-coding, statistical analysis, visualization and high-level reporting in a coherent and an entirely inter-operable manner.

The construct model described above is embedded in software, aptly named Construct. The software is designed to give maximum flexibility and control to the experimenter; the execution of the software is controlled by a parameters data file that gives direct access to practically every variable in the software, allowing the experimenter maximum flexibility in fine-tuning their experiments and adjusting their theoretic assumptions. The input parameters 
are as straightforward as specifying the number of agents and as detailed as specifying the specific topology if their ego- social and personal-knowledge networks. As it becomes relevant, variables can have initial and settings that change over time. Moreover, variables can be randomized according to randomization parameters provided by the researcher. For example, the knowledge-forgetting probability setting can be set to a distribution of values that are randomly selected for each time period based on a specific mean and variance value.

Construct software is freely available from CMU and runs on Linux and Windows operating systems. It has been run on basic laptop computers as well as sophisticated supercomputers. It can be obtained by visiting www.casos.cs.cmu.edu/construct. The software is written in $\mathrm{C}++$ is highly optimized for speed. Currently, the tool is developed for batch-execution only, although a graphical user interface wrapper is under development and internet-accessible distributed APIs are operational. 


\section{APPENDIX D: CONSTRUCT SOFTWARE \& CONFIGURATION}

The computer scripting software that handles the preparation, execution and data processing of the experiments is listed in detail in this chapter. Processing occurs in two steps: (1) construct the input script for CONSTRUCT execution and submit the script to the CONDOR computation networks for execution 100 times, then (2) transform the raw output from CONSTRUCT into datafiles for the analysis tools, in this case MATLAB. The program submitBuildAndCondor.pl initiates the CONSTRUCT build and run. The program runMultipleGeneric.pl transforms the raw data into analysis-software-friendly format. These two scripts and all of the customized code underlying them are presented below. There are a few utility tools used in this process that are not included here as they are purely for non-scientific, data management activities, such as moving data from one computer to another, compressing and decompressing data files, and archiving data, etc. The scripts listed and described in the table below.

Table D-1. Software Scripts.

\begin{tabular}{|c|c|}
\hline submitBuildAndCondor .pl & $\begin{array}{l}\text { Top-level script for creating CONSTRUCT } \\
\text { input files and submitting CONSTRUCT for } \\
\text { execution of the simulation }\end{array}$ \\
\hline buildExperiment.pl & $\begin{array}{l}\text { Manages the high-level specifications for the } \\
\text { experiment batches by calling buildScript.pl }\end{array}$ \\
\hline submitCondor .pl & $\begin{array}{l}\text { Creates and submits the CONDOR execution } \\
\text { script. Contains instructions to run } \\
\text { CONSTRUCT. }\end{array}$ \\
\hline buildScript.pl & 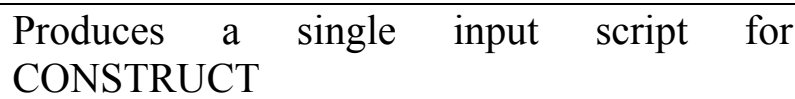 \\
\hline lib.pl & General utilities library for all scripts. \\
\hline buildRpt.pl & $\begin{array}{l}\text { Does the conversion from a single } \\
\text { CONSTRUCT output file into multiple data } \\
\text { formats for further analysis, mostly in CSV } \\
\text { format. }\end{array}$ \\
\hline runMultipleGeneric.pl & $\begin{array}{l}\text { Top-level script for transforming the } \\
\text { CONSTRUCT output datafile into other files } \\
\text { in formats for use by data analysis programs }\end{array}$ \\
\hline
\end{tabular}

D-1 


\begin{tabular}{|l|l|}
\hline runComputes.pl & $\begin{array}{l}\text { Combines data output from individual } \\
\text { replications into per-experiment data files. }\end{array}$ \\
\hline CSV.pm & Library for handling CSV-formatted data files \\
\hline kNetwork.pm & $\begin{array}{l}\text { Returns columnized data from a CSV } \\
\text { formatted data file. }\end{array}$ \\
\hline knowledgeRowColMap.pm & Manages rows of knowledge network data. \\
\hline dataFeed.pm & Handles data for an individual actor. \\
\hline smartDataFeed.pm & $\begin{array}{l}\text { Handles data for an actor, either side of the } \\
\text { information exchange. }\end{array}$ \\
\hline dataStore.pm & Moves datasets around system. \\
\hline $\begin{array}{l}\text { computeBin/ } \\
\text { createSmartDataFeedCSV.pl }\end{array}$ & $\begin{array}{l}\text { Extracts the delta between two data networks } \\
\text { (time periods). }\end{array}$ \\
\hline $\begin{array}{l}\text { computeBin/ } \\
\text { culturePctAllTpFromDataFeed.pl }\end{array}$ & $\begin{array}{l}\text { Extracts Cultural data delta between two data } \\
\text { networks (time periods). }\end{array}$ \\
\hline $\begin{array}{l}\text { computesBin/ } \\
\text { culturePct.pl }\end{array}$ & $\begin{array}{l}\text { Computes the percentage of culture diffused } \\
\text { throughout a network. }\end{array}$ \\
\hline $\begin{array}{l}\text { computesBin/ } \\
\text { culturePctAlltp.pl }\end{array}$ & $\begin{array}{l}\text { Computes the percentage of culture diffused } \\
\text { throughout a network by teams. }\end{array}$ \\
\hline
\end{tabular}

submitBuildAndCondor.pl

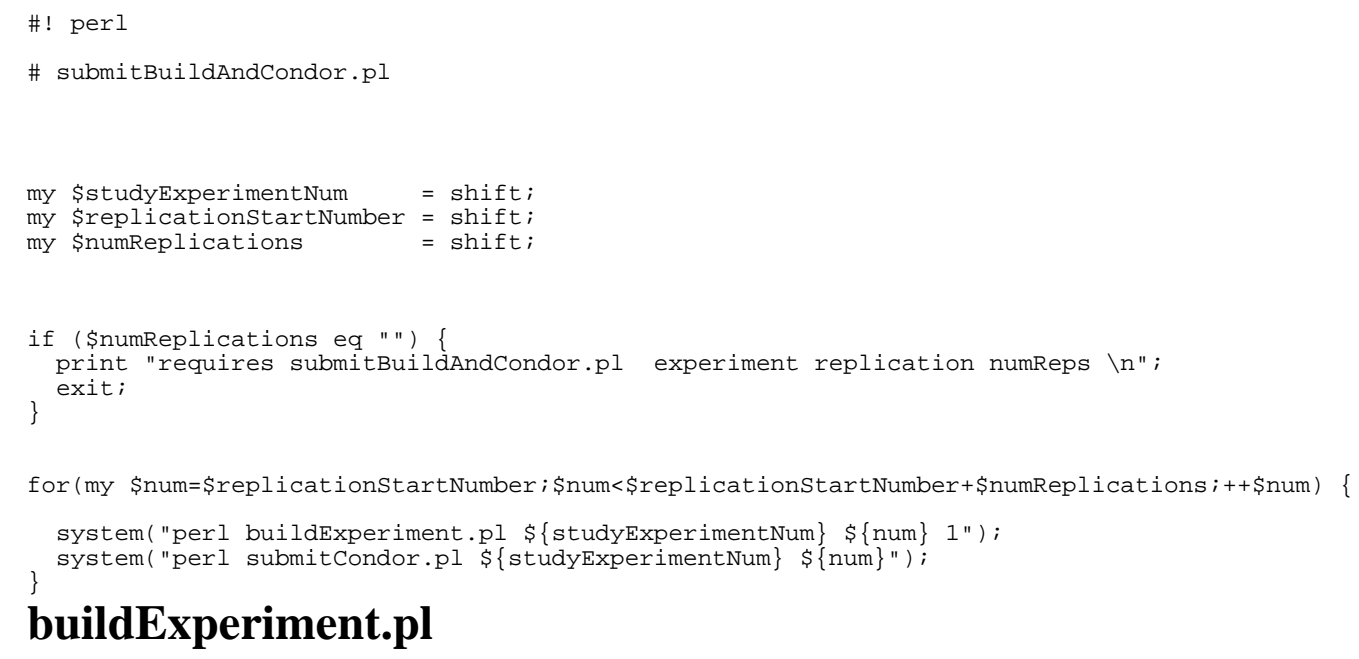

buildExperiment.pl

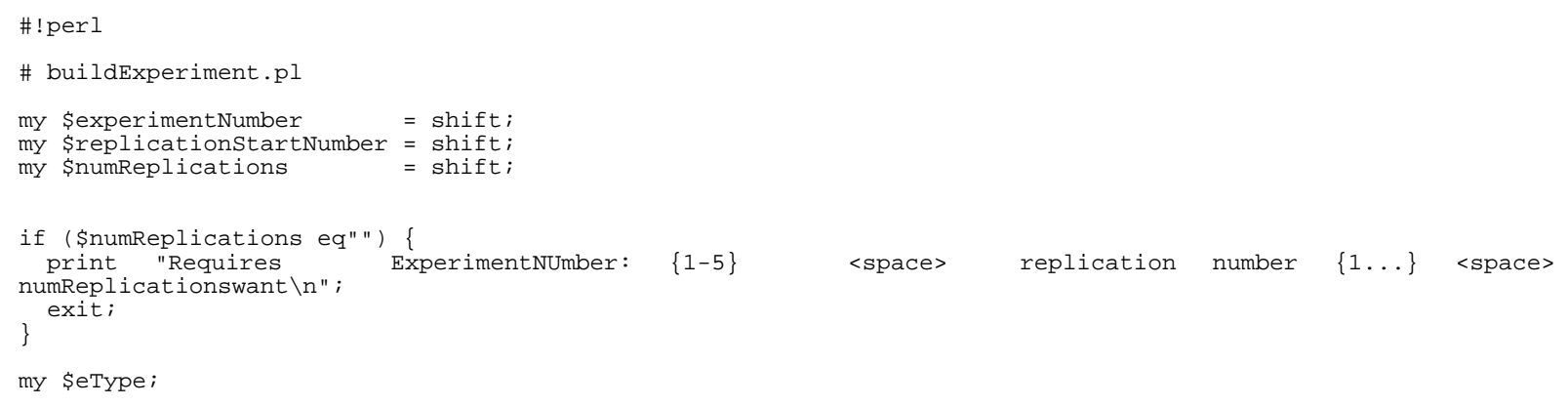

D-2 
my@timePeriodsList ;

my @teamsPerorgBothList;

my @agentsPerTeamList

my @agentsPerTeamBetaList;

my @knowledgePerTeamList

my @knowledgePerTeamBetaList :

my @actorSpecializationList

my @actorspecializationBetaList :

my @teamInterdepencelist.

my @teamInterdepenceBetaList :

my @willingnessToSeekList;

my @willingnessToSeekBetaList;

my @willingnessToShareList;

my @willingnessToShareBetaList;

my @orgCultureComplexityList

my @orgculturecomplexityBetaList:

my @teamculturecomplexityList;

my @teamcultureComplexityBetaList

my @cultureoutreachAlpaList

my @cultureoutreachBetaList;

my @taskFocusList ;

my @taskFocusBetaList;

my \$exe = 'buildScript.pl';

\#\$еType= "production_all";

\# \$етуре= "production_oneMid" ;

\$eType = "prodExp $\$\{$ experimentNumber $\} "$;

\#

\#\$eType = "production_exp2".

\# \$eType = "production_exp3".

\# \$еType = "production exp4"

\# \$еType = "production_exp5",

\#\$еType= "smalloneTeam";

\#\$еType= "smallTwoTeam".

\#\$еType= "testMax";

\#

\#\#\# set up all as a default for the midpoint

@timePeriodsList $=$ qw $/ 500 /$;

\#@timePeriodsList $=$ qw $/ 5 /$;

@timePeriodsList $=$ qw $/ 1000 /$

\#@timePeriodsList $=$ qw $/ 5000 /$;

@teamsPerorgBothList $=$ qw $/ 6 /$

@agentsPerTeamList $=\mathrm{qw} / 10 /$

@agentsPerTeamBetaList $=$ qw $/ 1.0 /$

$@$ knowledgePerTeamList $=$ qw $/ 30 /$

$@$ knowledgePerTeamBetaList $=$ qw $/ 1.0 /$;

@actorSpecializationList $=q w / 1.0 /$

@actorspecializationBetaList $=$ qw $/ 1.0 /$

@teamInterdepenceList $=\mathrm{qw} / \odot .3 /$;

@teamInterdepenceBetaList $=$ qw $/ 1.0 /$

@willingnessToSeekList = qw/ $0.4 \%$;

@willingnessToSeekBetaList = qw/ 1.0/;

@willingnessToShareList $=q w / 0.4 /$

QwillingnessToShareBetaList $=q w / 1.0 /$;

@orgCultureComplexityList = qw $/ 30 /$ :

@orgcultureComplexityBetaList $=\mathrm{qw} / 1.0 /$

@teamculturecomplexitylist $=\mathrm{qw} / 6 \%$

@teamcultureComplexityBetaList $=$ qw $/ 1.0 /$;

@culture0utreachList $=$ qw $/ 0.4 /$;

@cultureoutreachBetaList $=$ qw $/ 1.0 /$;

@taskFocusList $=\mathrm{qw} / \mathrm{M} /$;

@taskFocusBetaList $=q \mathrm{w} / \mathrm{M} /$;

if (\$eType eq "production_all") \{

\$numReplications $=1$;

@timePeriodsList $=$ qw $/ 500 / ;$

@teamsPerorgBothList = qw /2610/;

D-3 


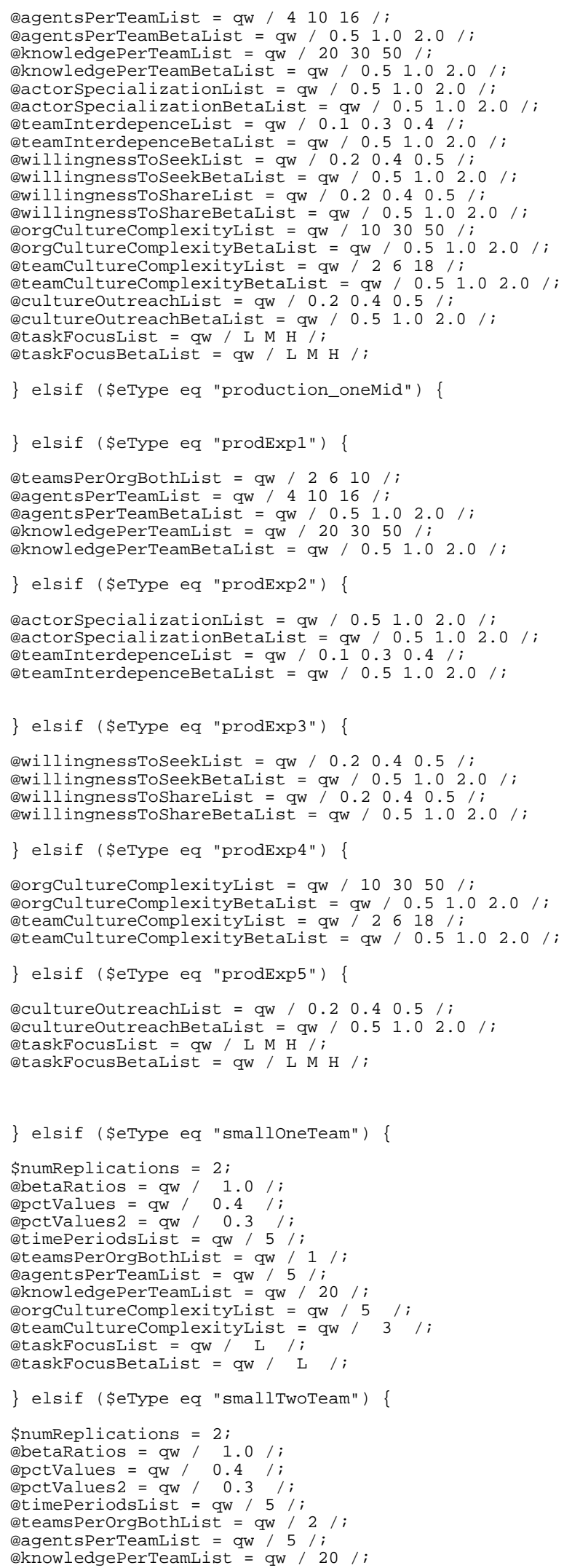




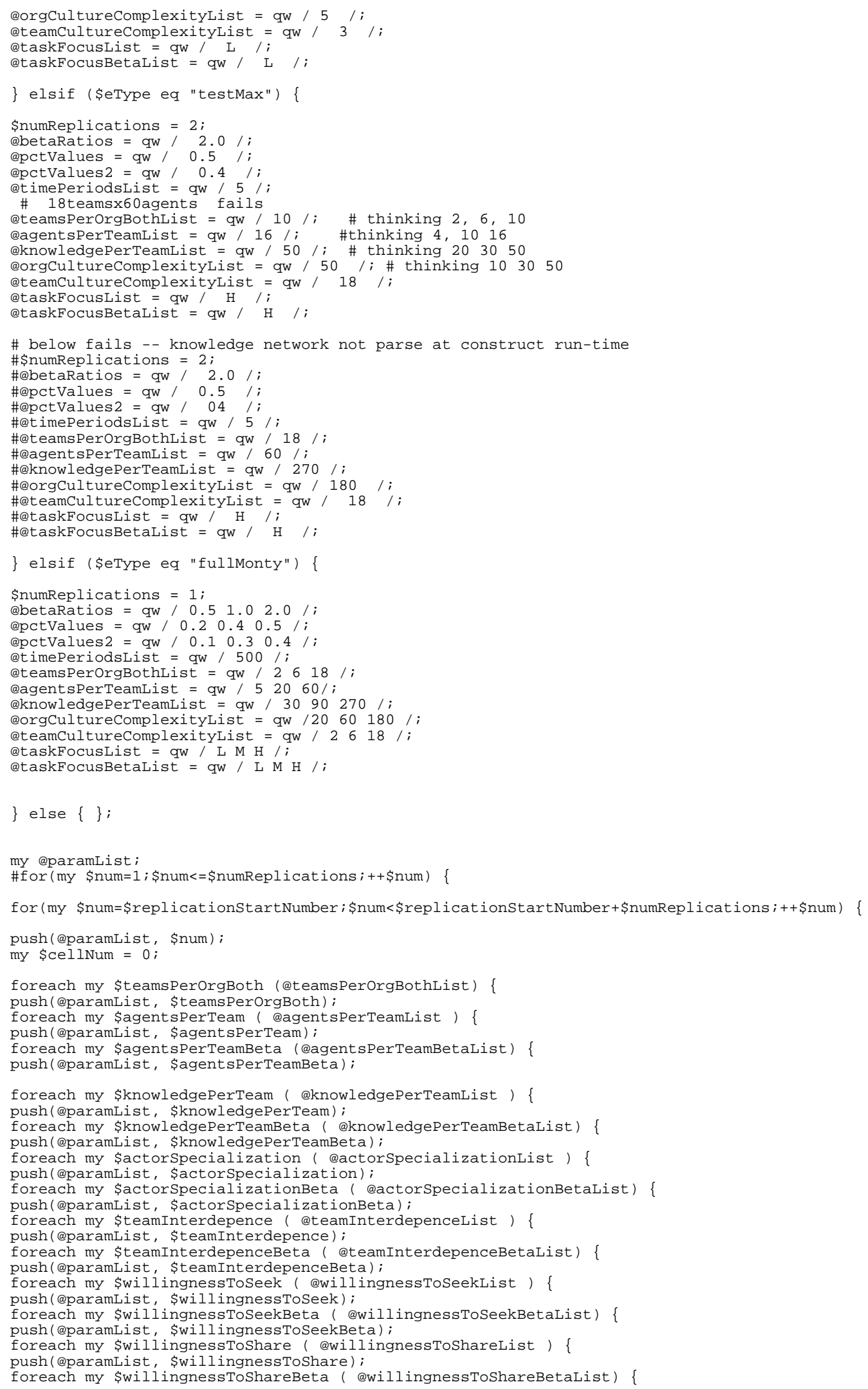

\section{D-5}




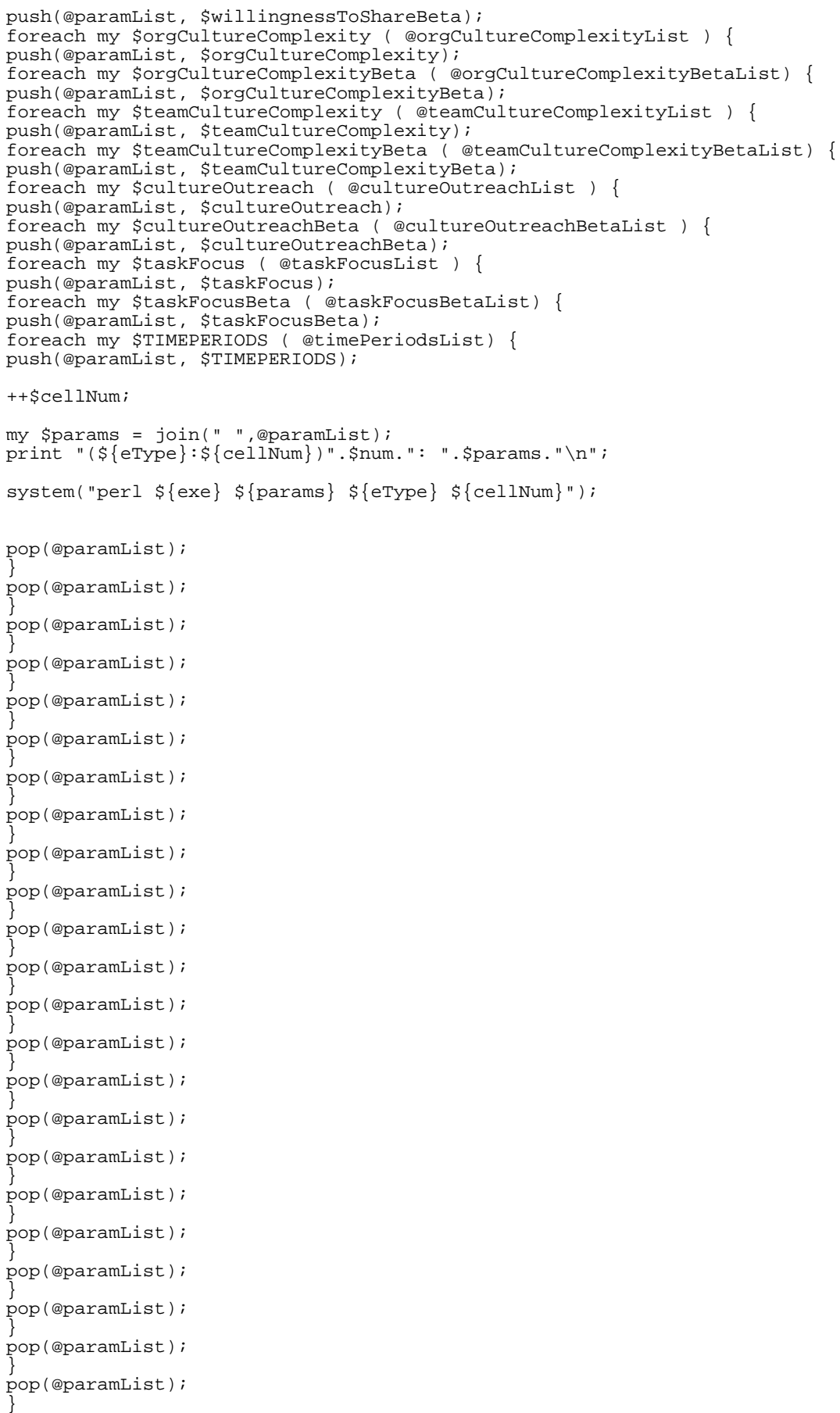

\section{submitCondor.pl}

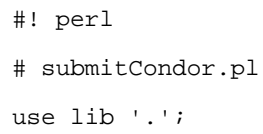




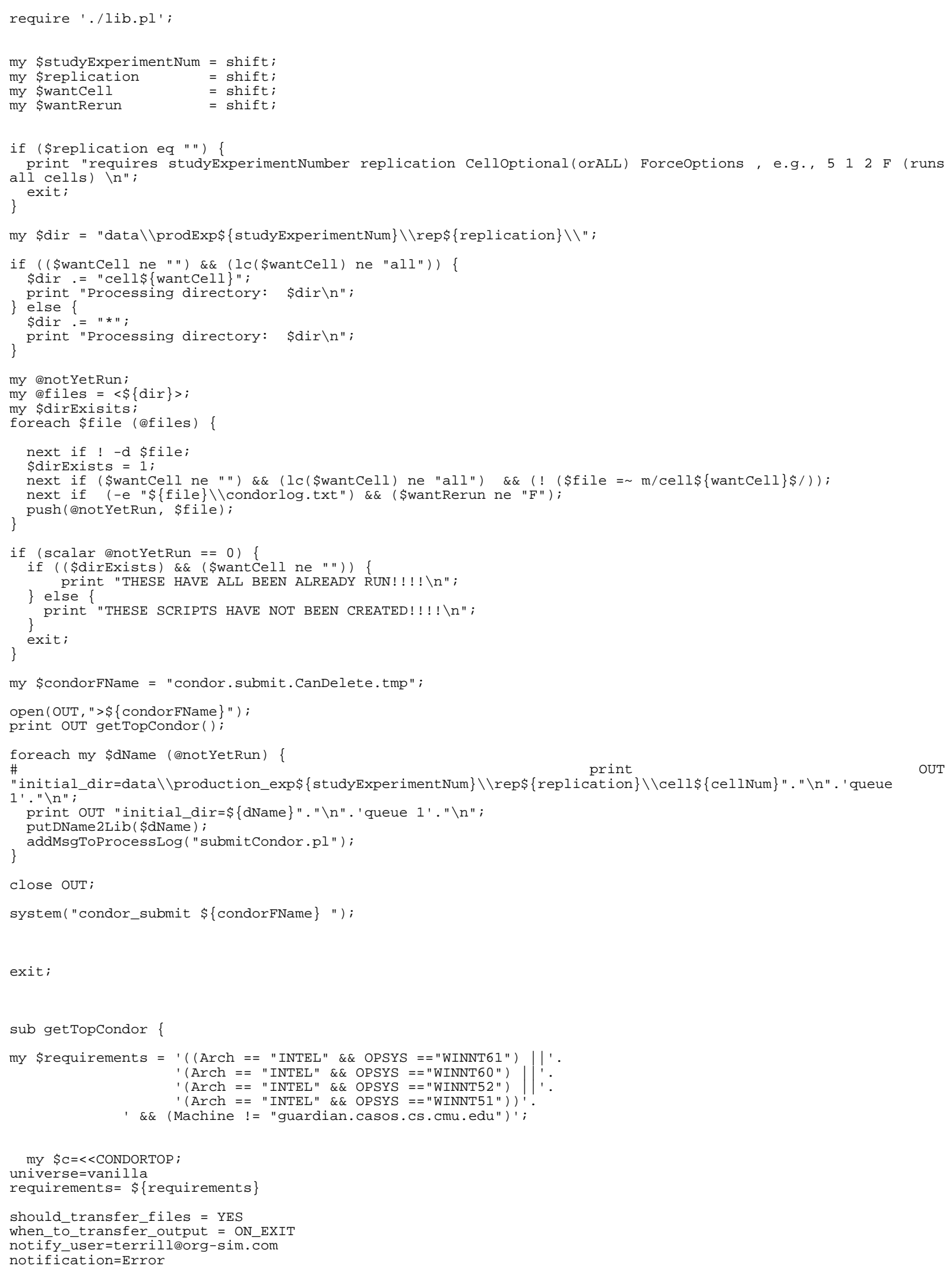

\section{D-7}




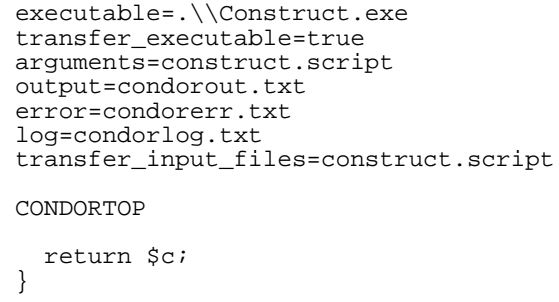

\section{buildScript.pl}

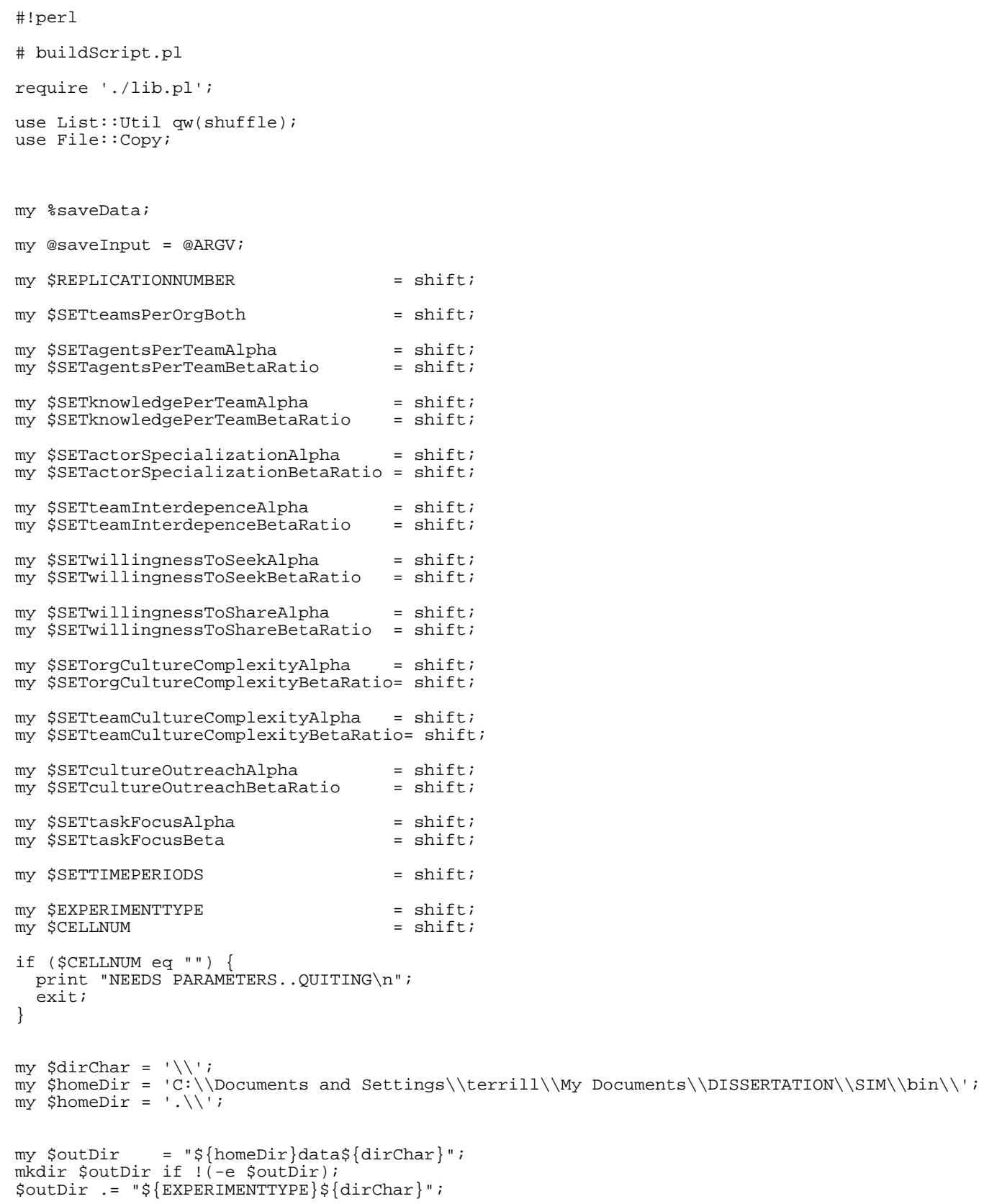

\section{D-8}




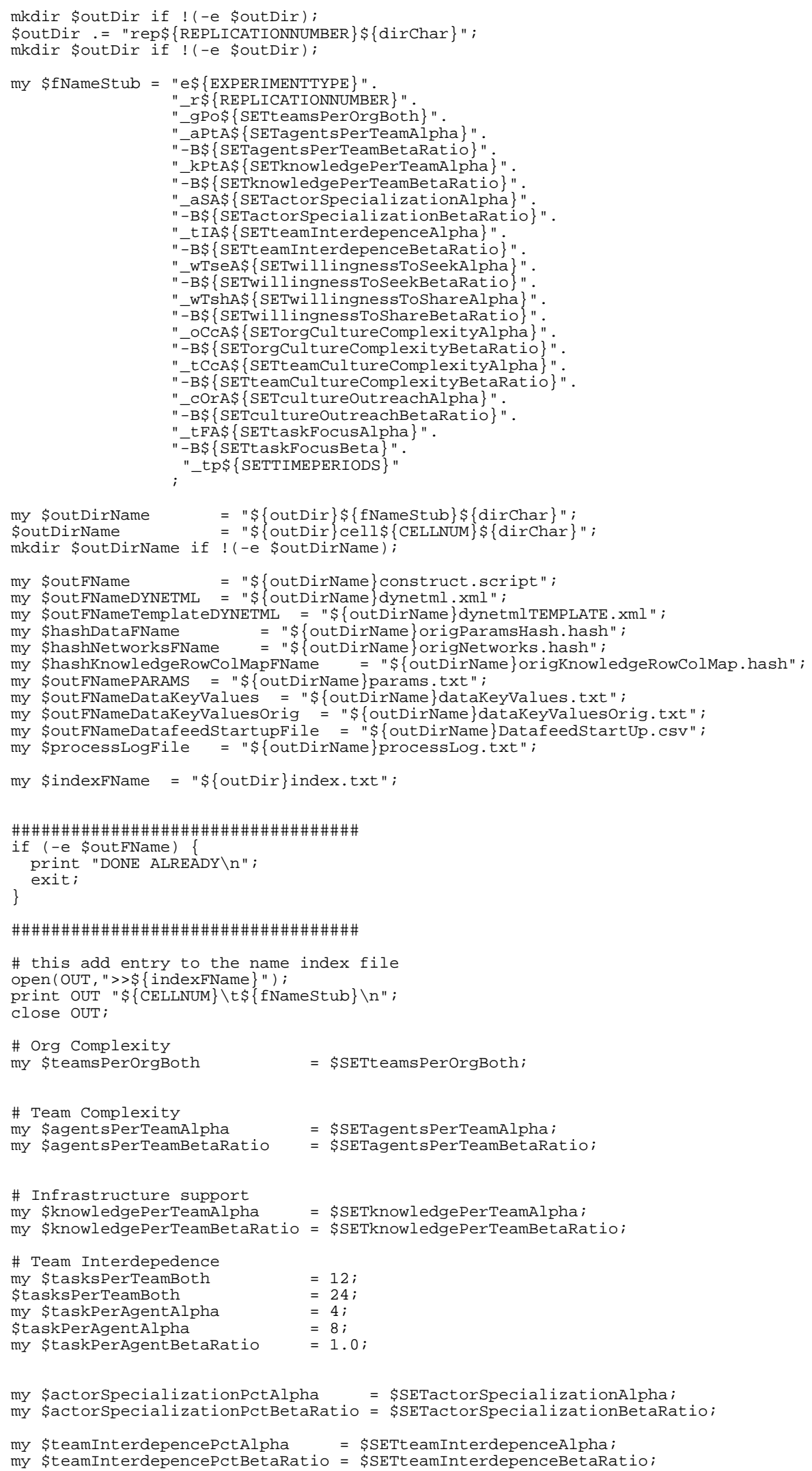




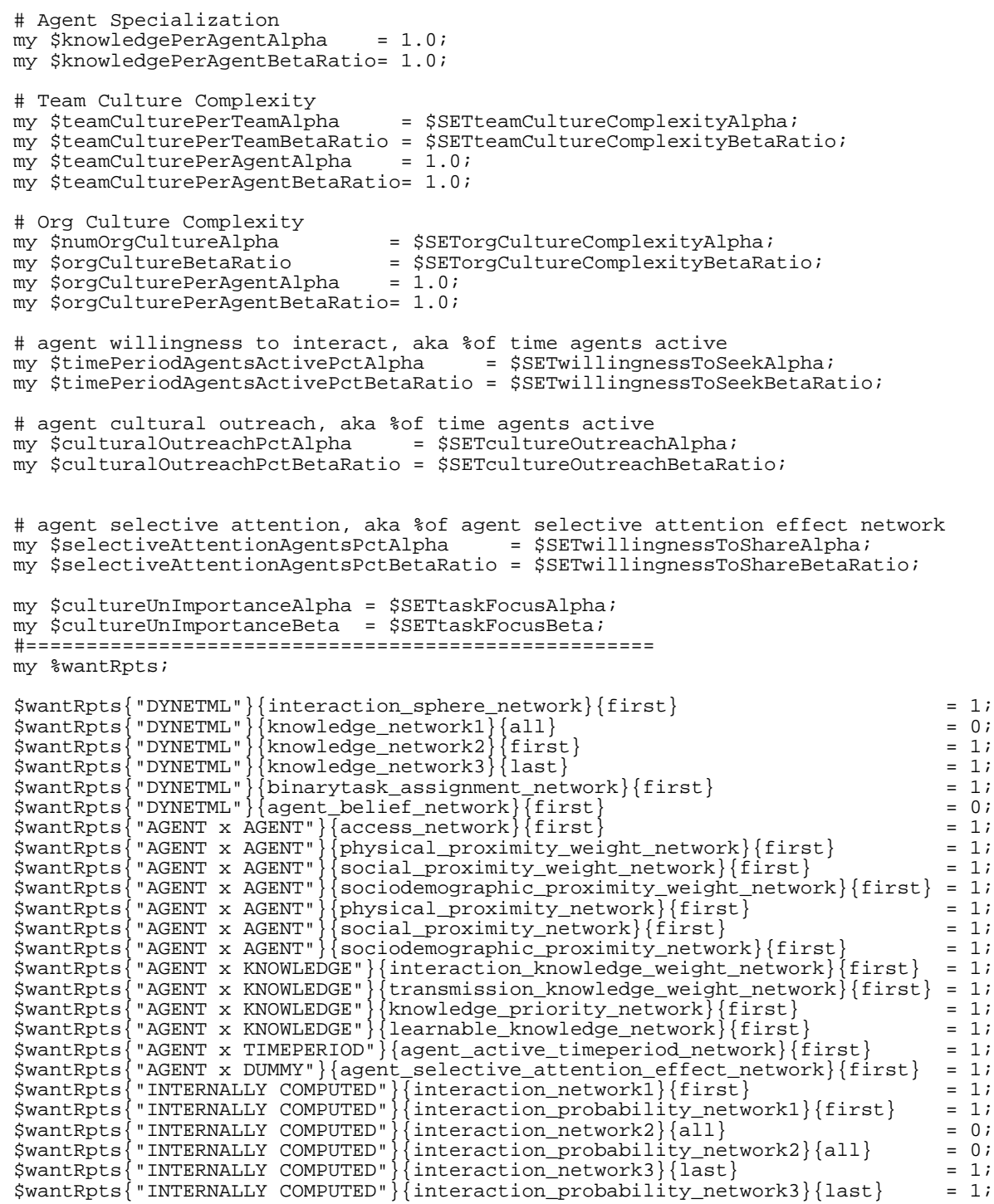




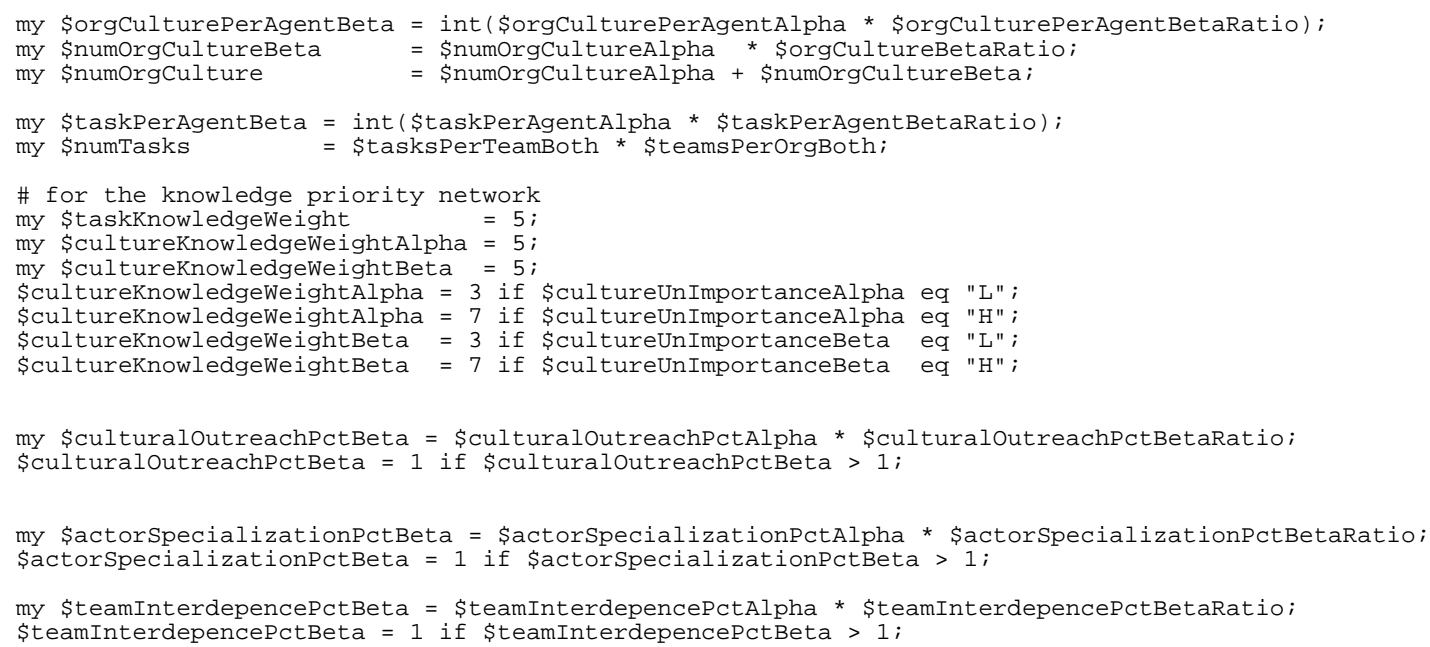




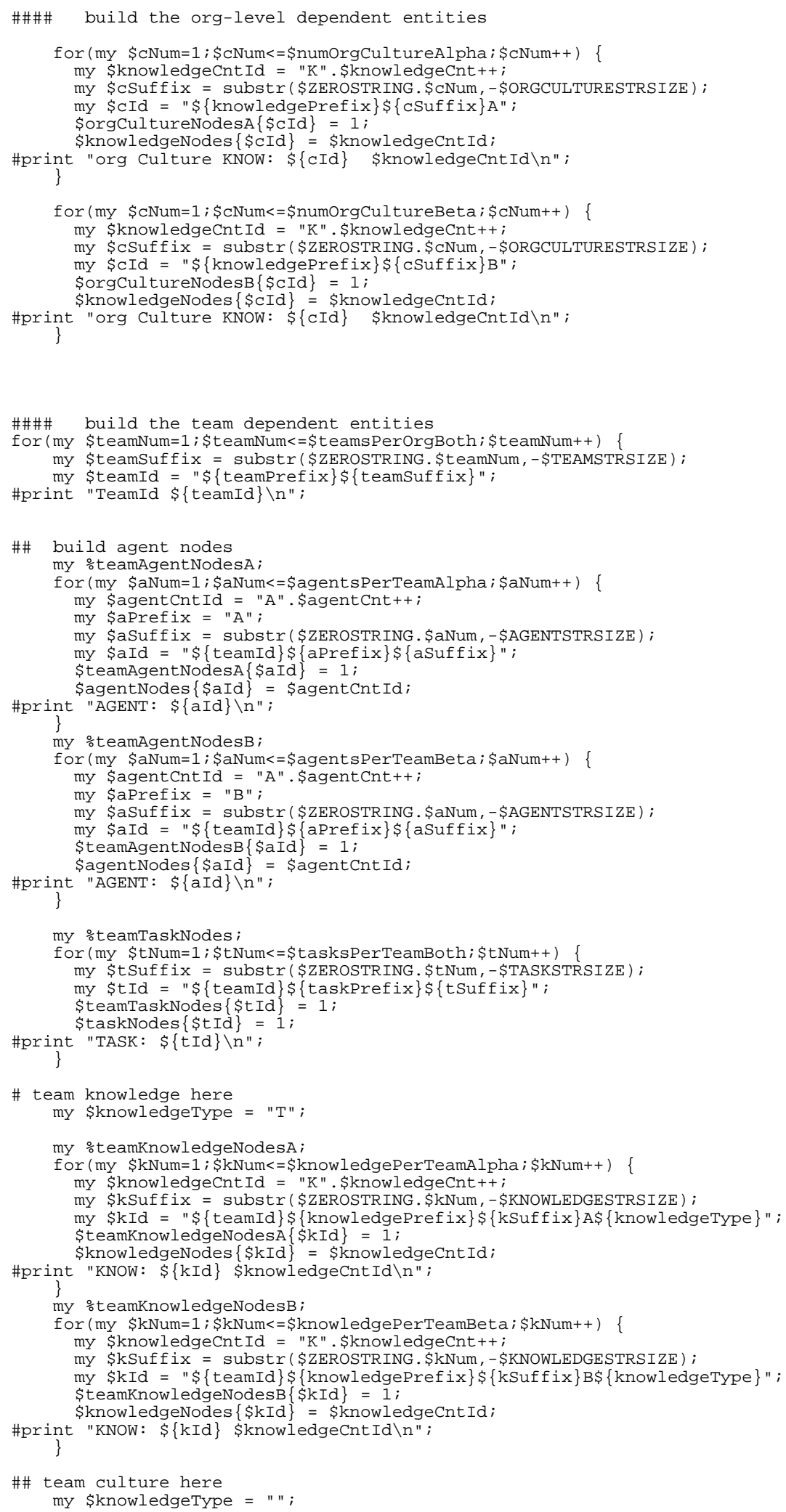




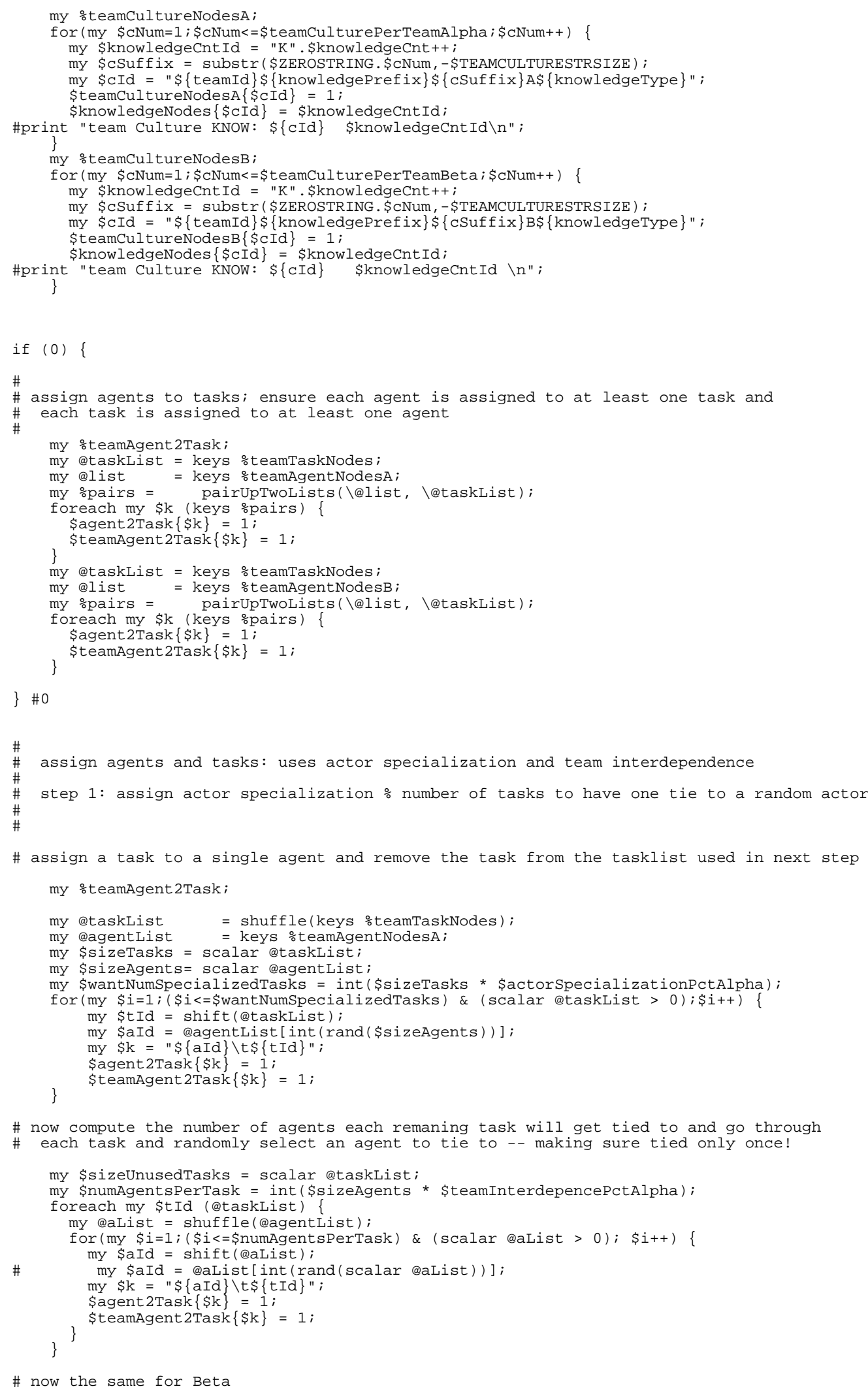




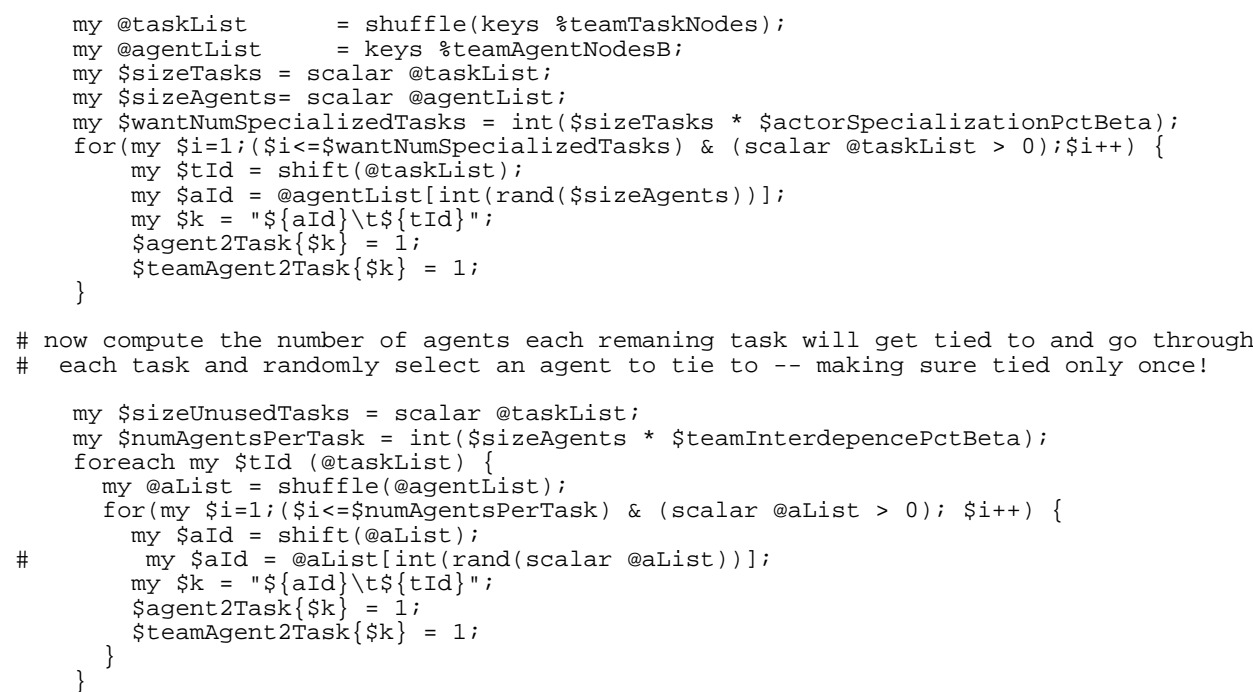

D-14 


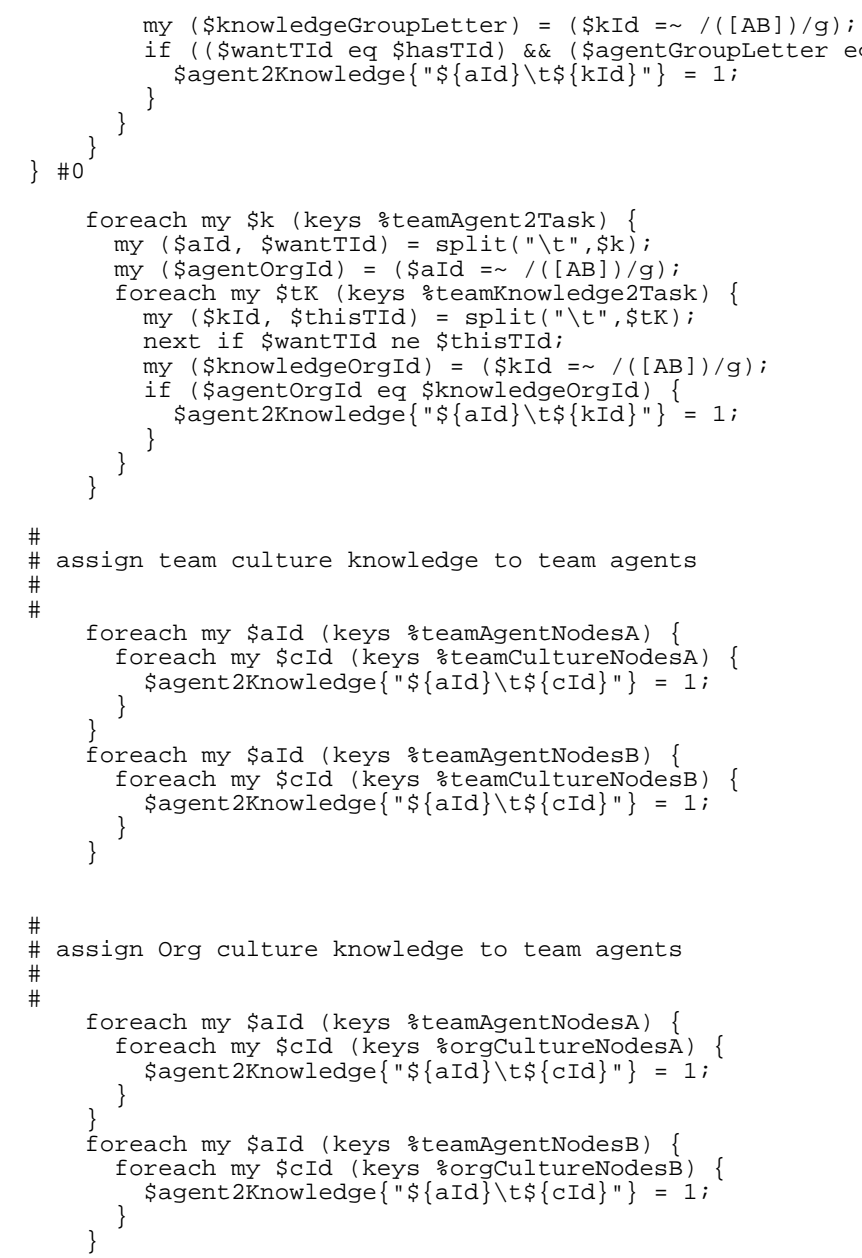

\section{D-15}




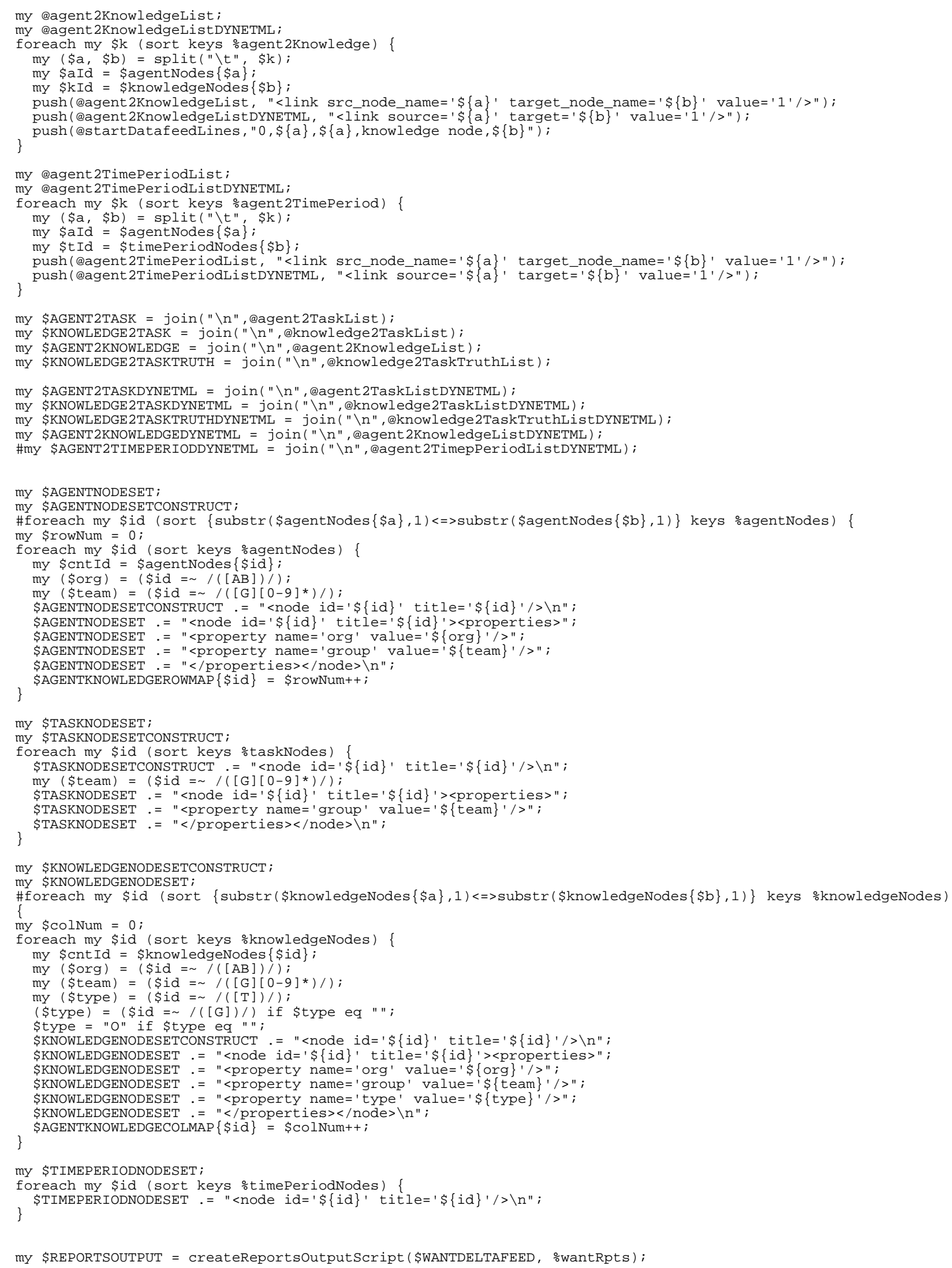

\section{D-16}




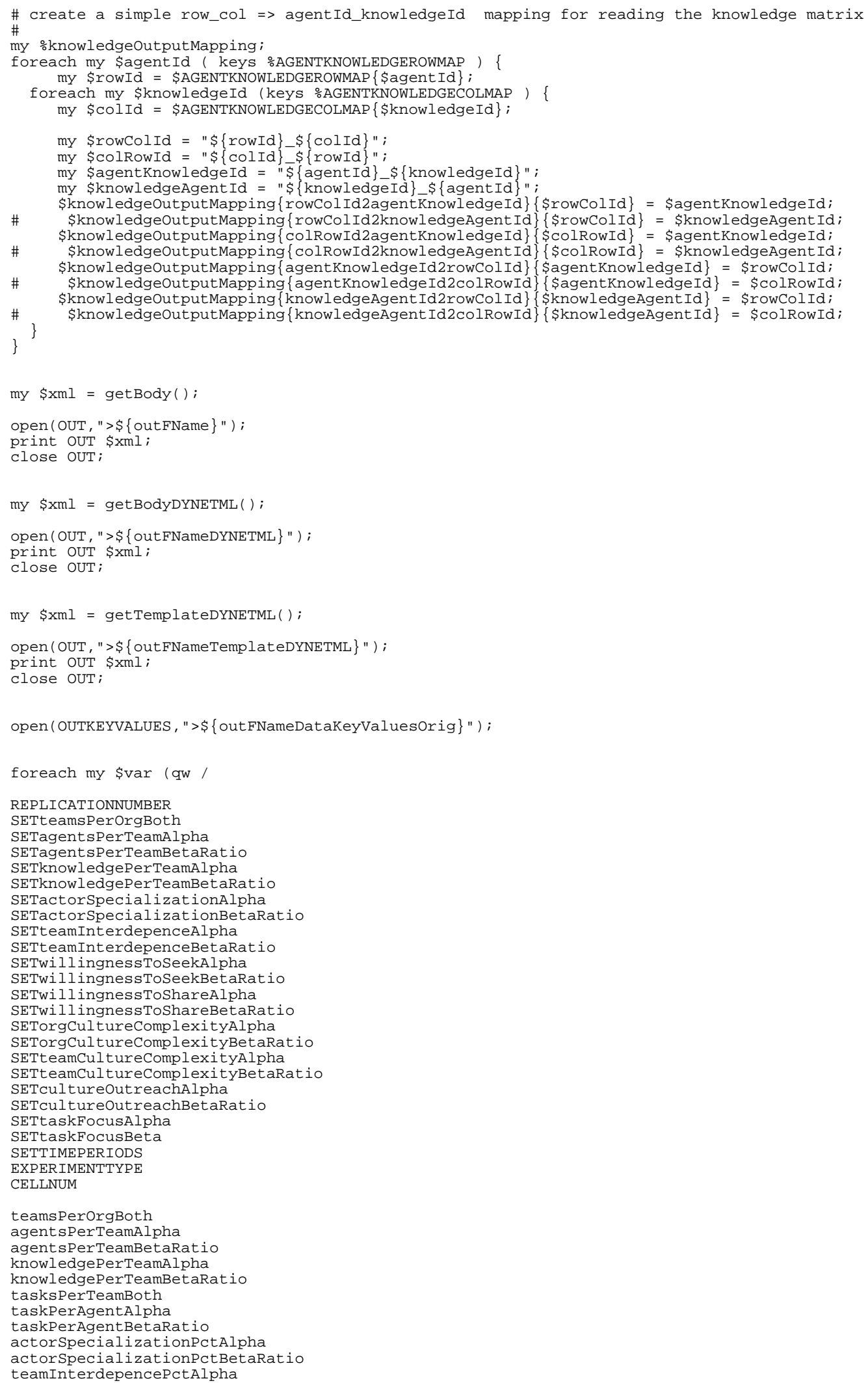




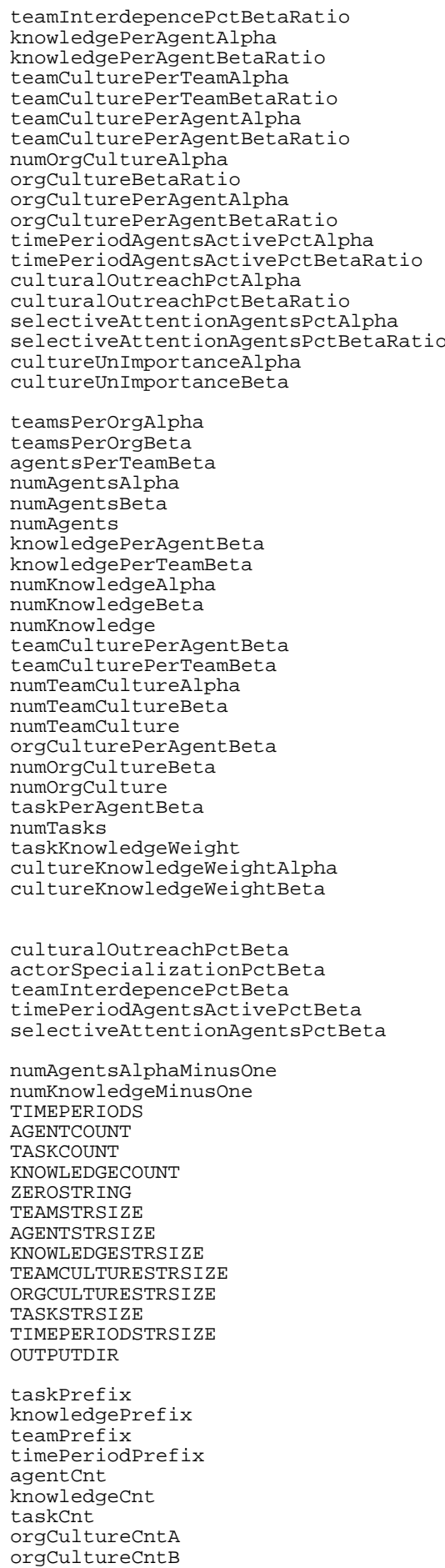

\section{/) \{}

my $\$$ stmt $=$ "\\$saveData $\{\$\{$ var $\}\}=\backslash \$ \$\{$ var $\} "$;
eval $(\$$ stmt $) ;$

my \$val;

\$stmt $=$ '\$val $=' . " \backslash \$\{$ var $\} ; " ;$ eval (\$stmt); 


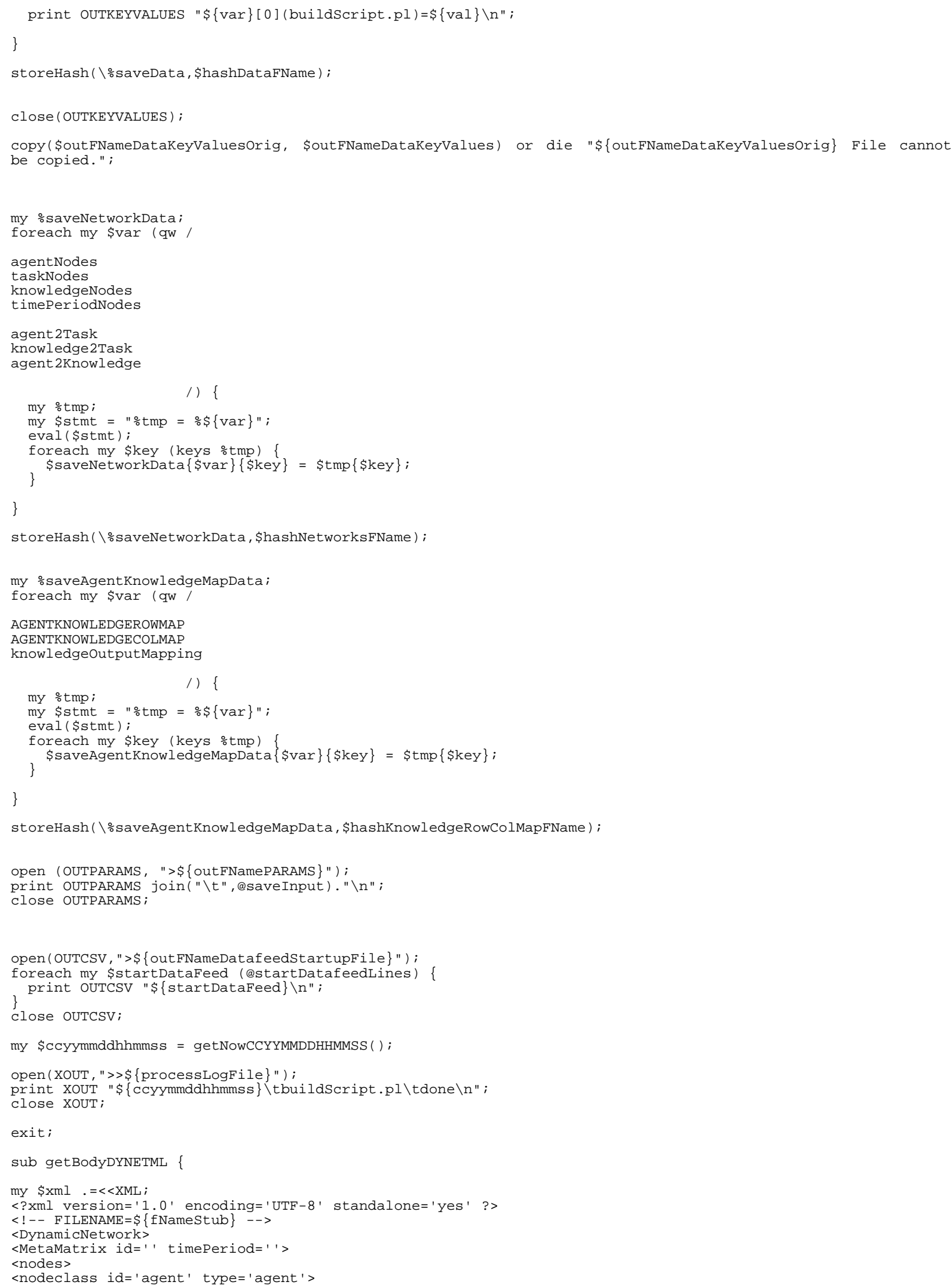




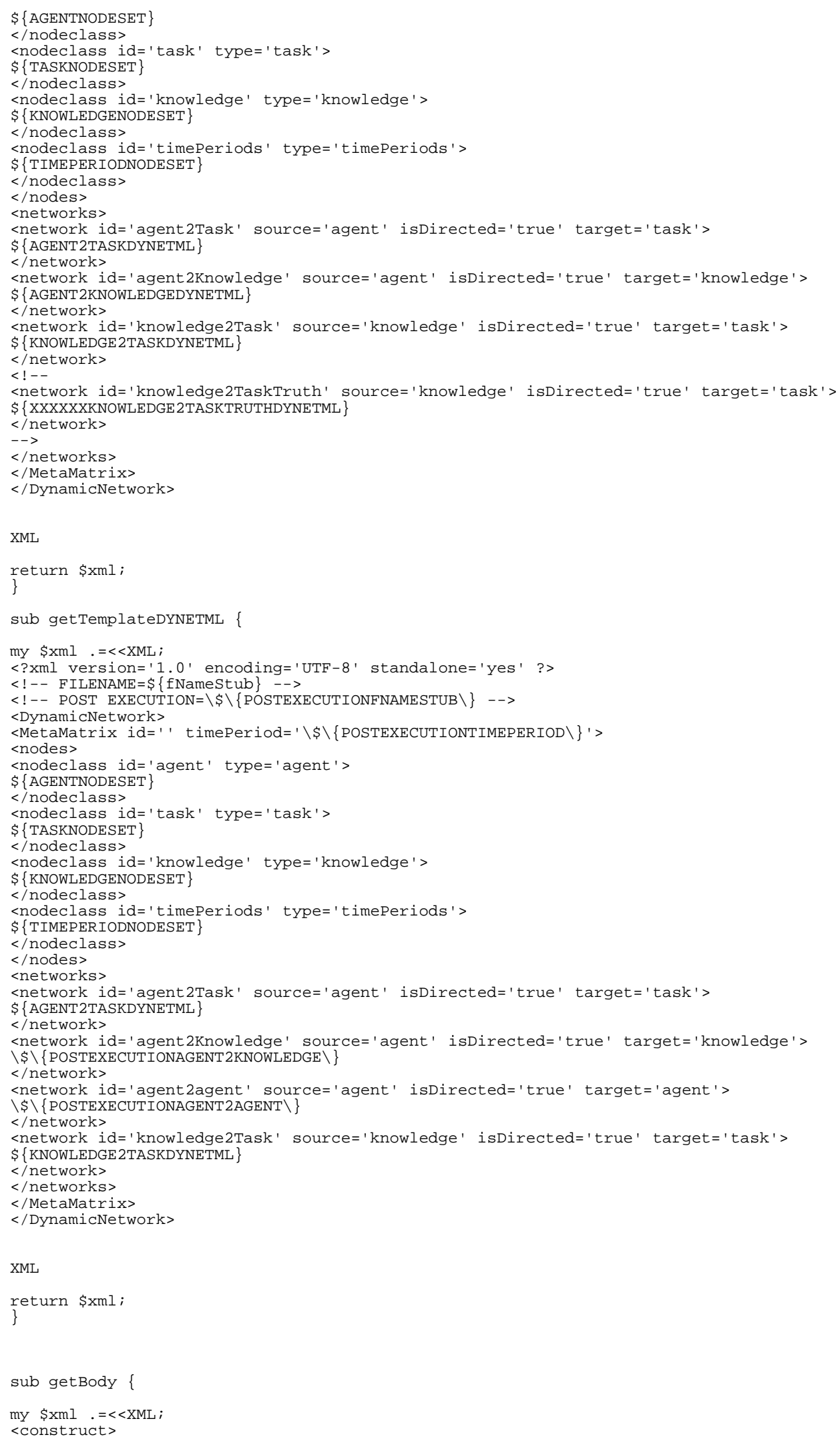




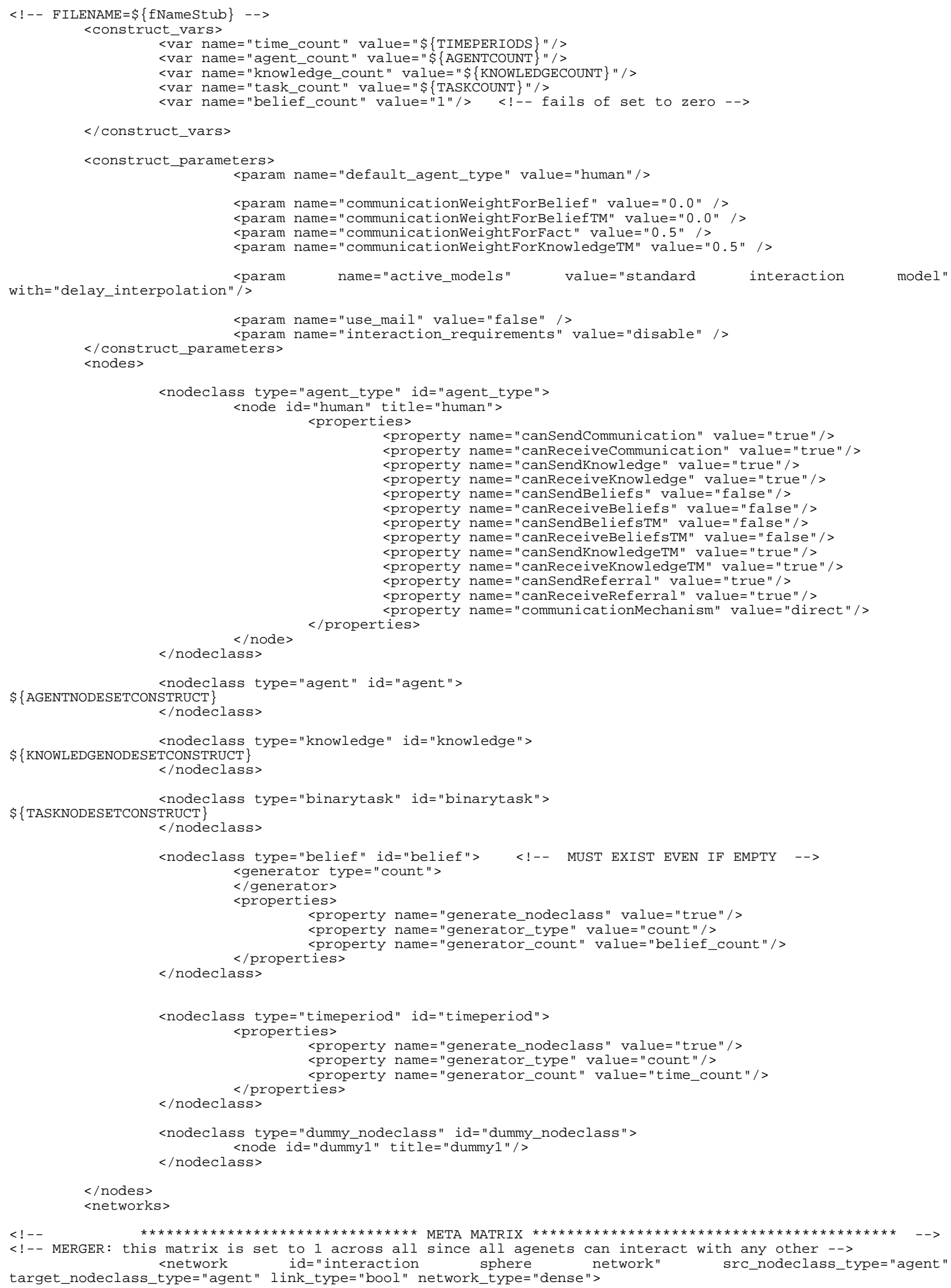

\section{D-21}




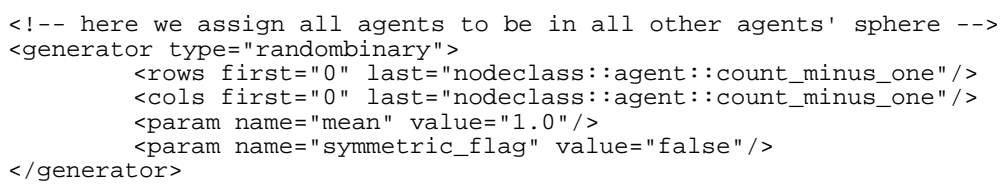




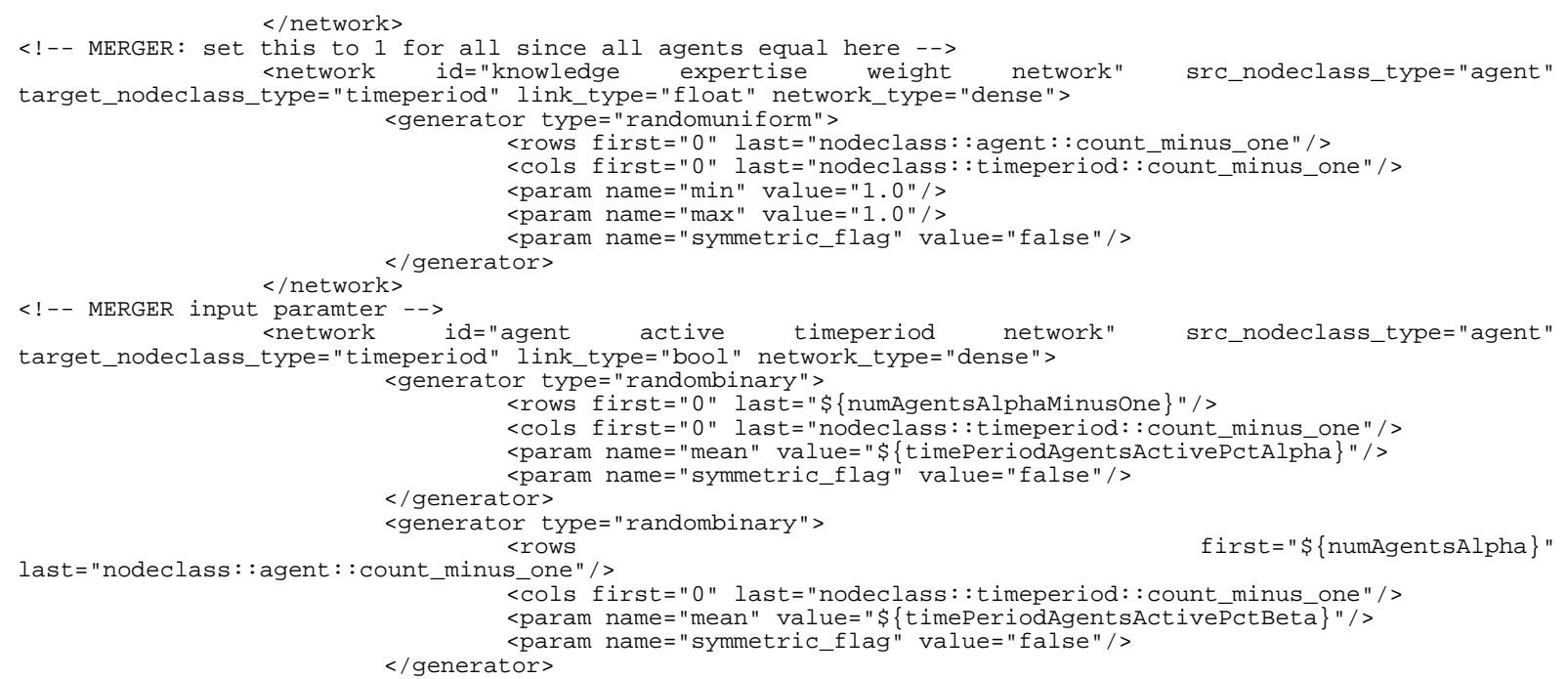

<!-- MERGER: set this to 1 for all since all agents equal here --> 


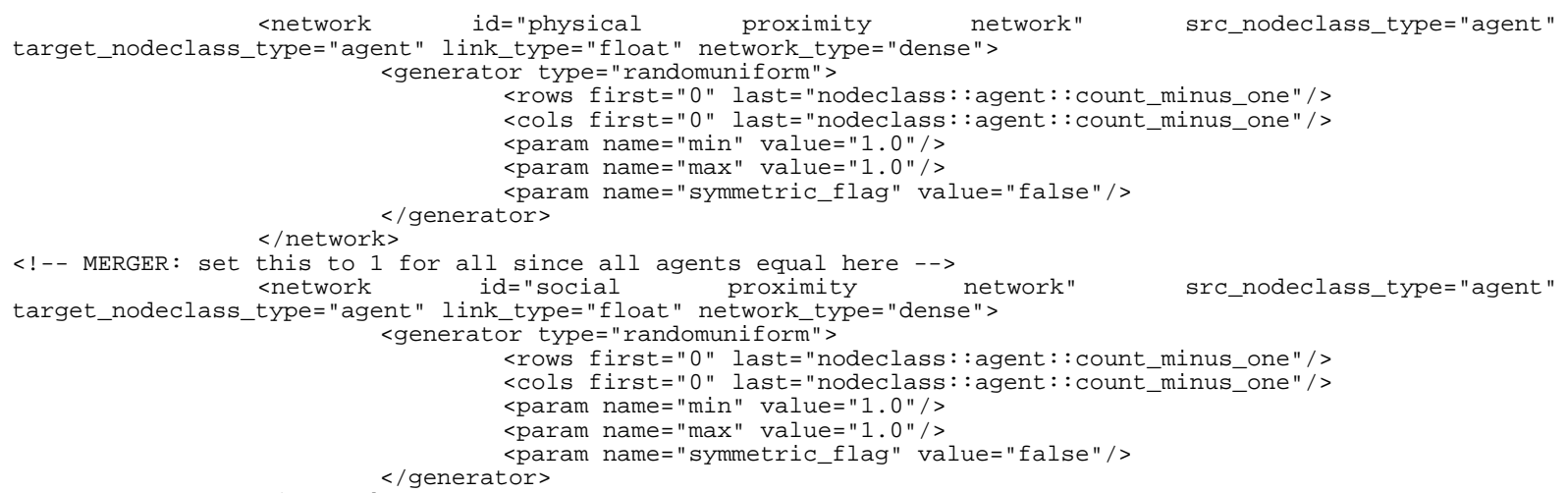


(param name="max" value="\$\{cultureknowledgeWeightBeta $\} " />$

$</$ network>

<param name="symmetric_flag" value="false"/>

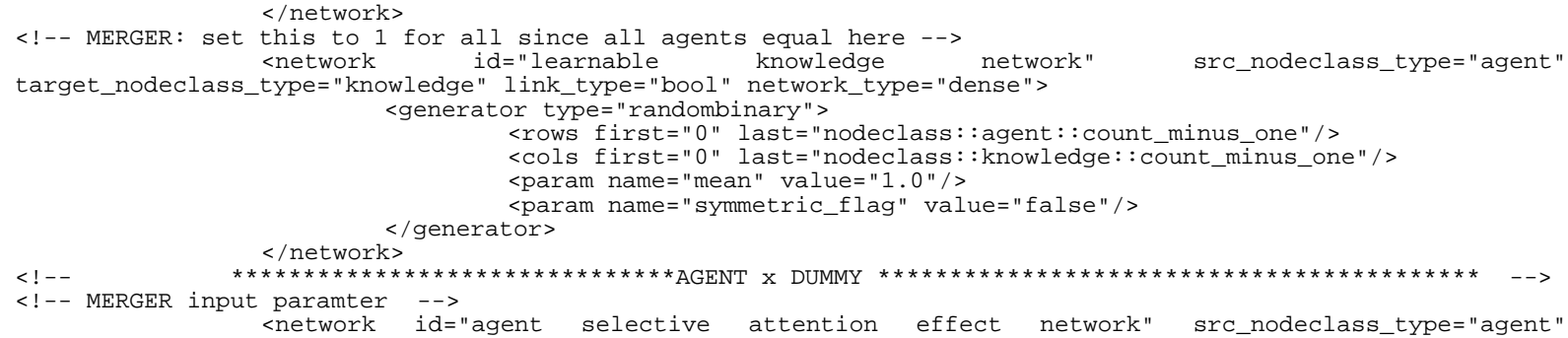




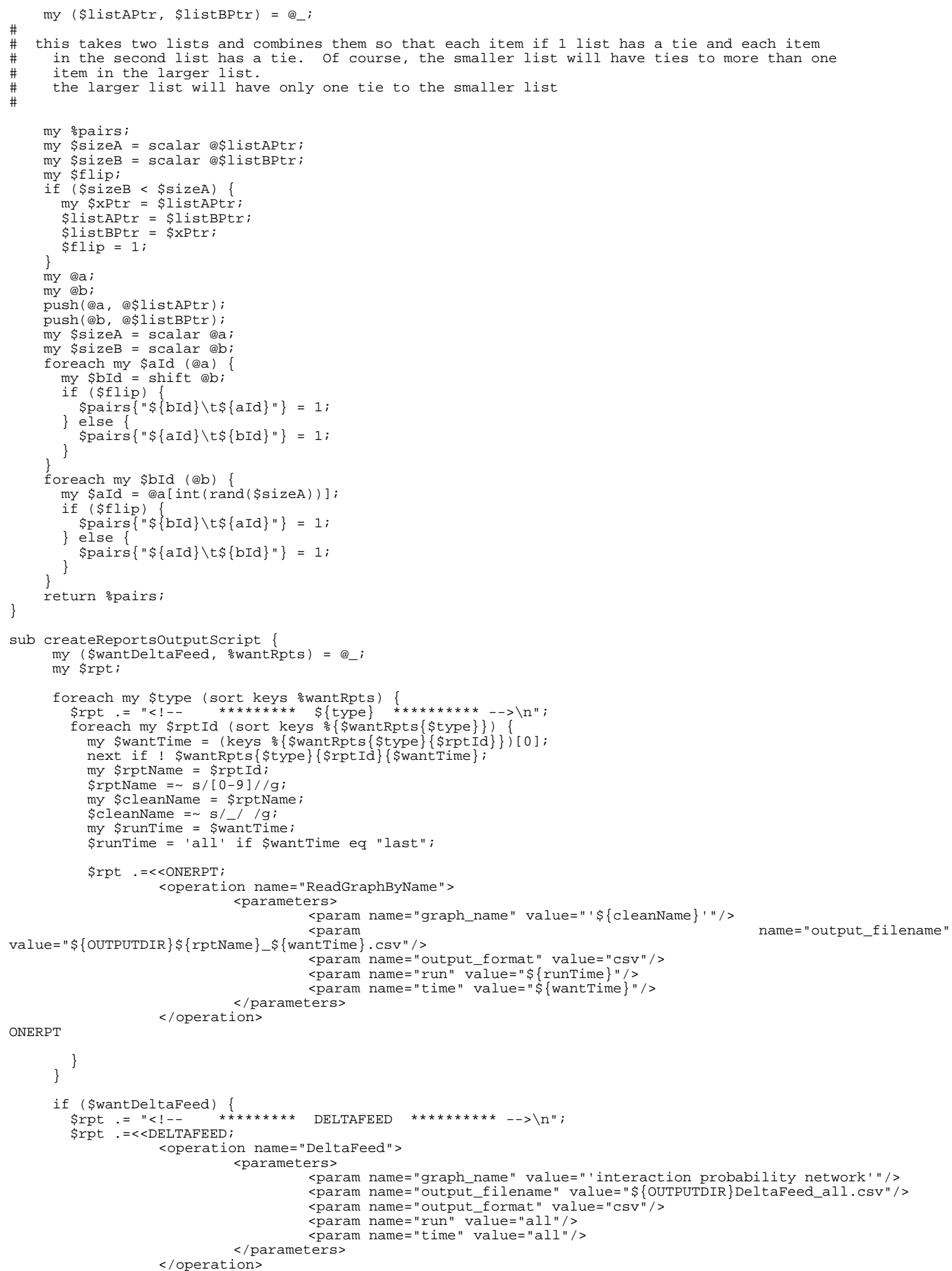

ONERPT

\}$^{3}$

\section{D-26}


DELTAFEED

return \$rpt;

\section{lib.pl}

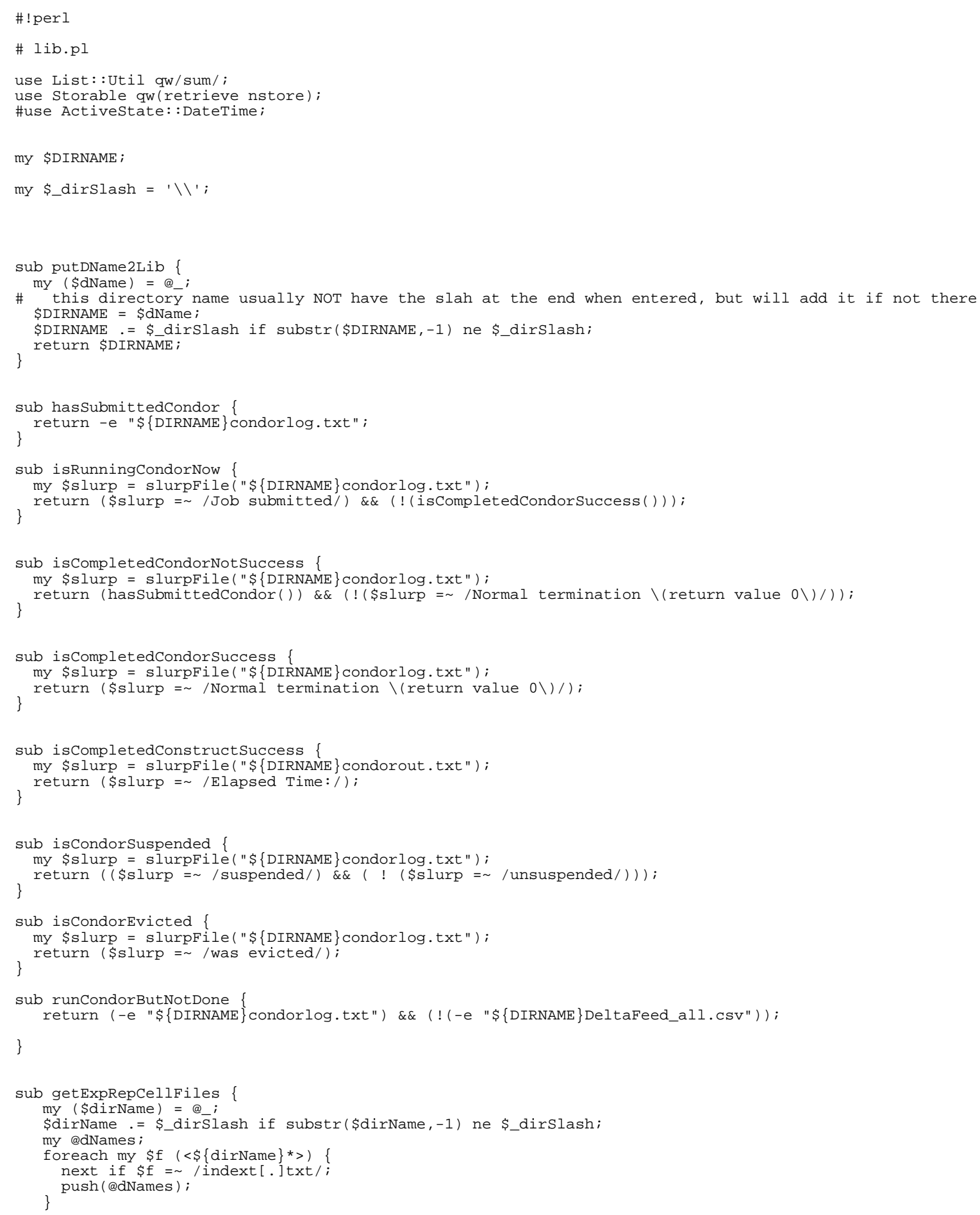




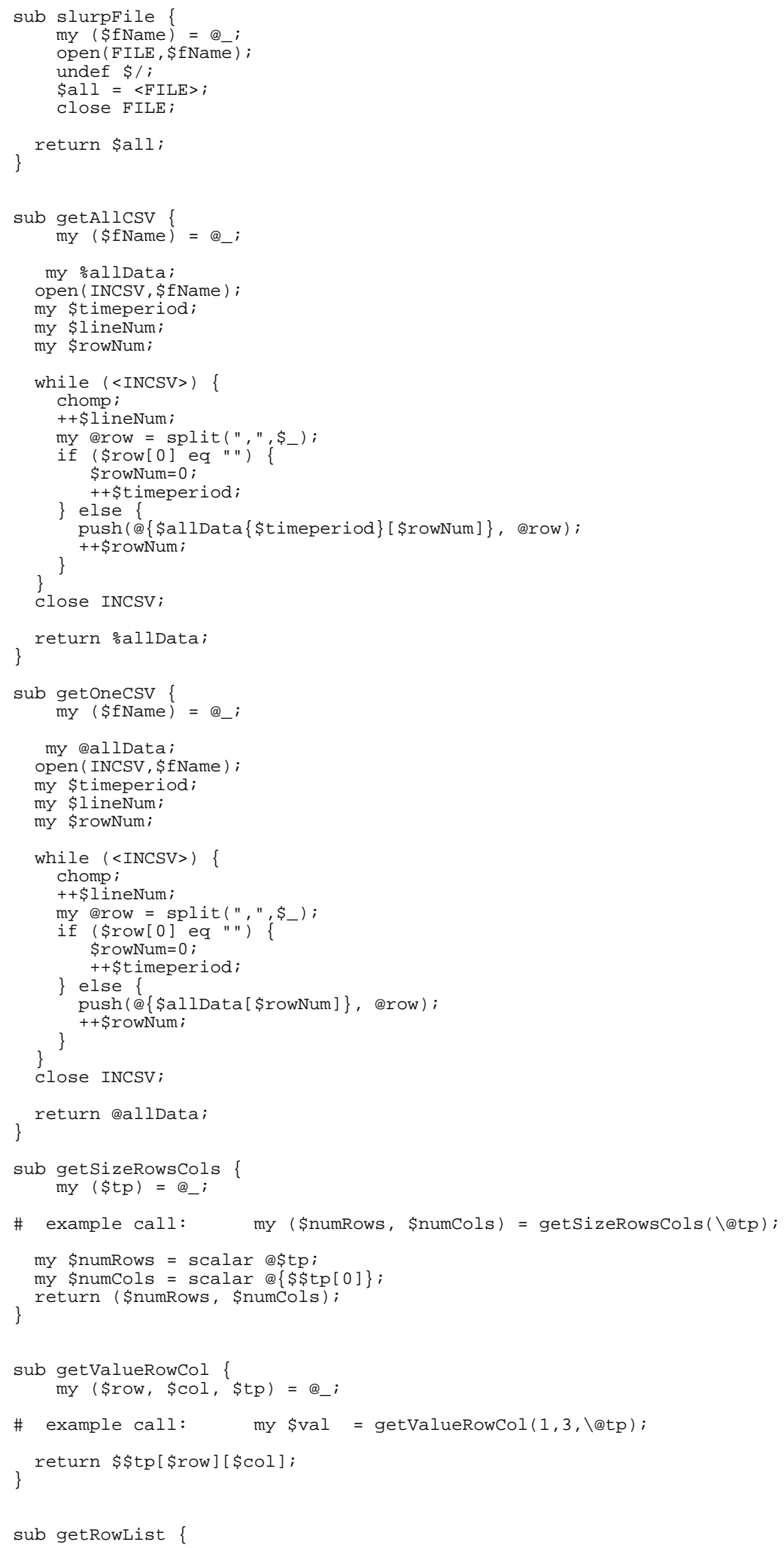

D-28 


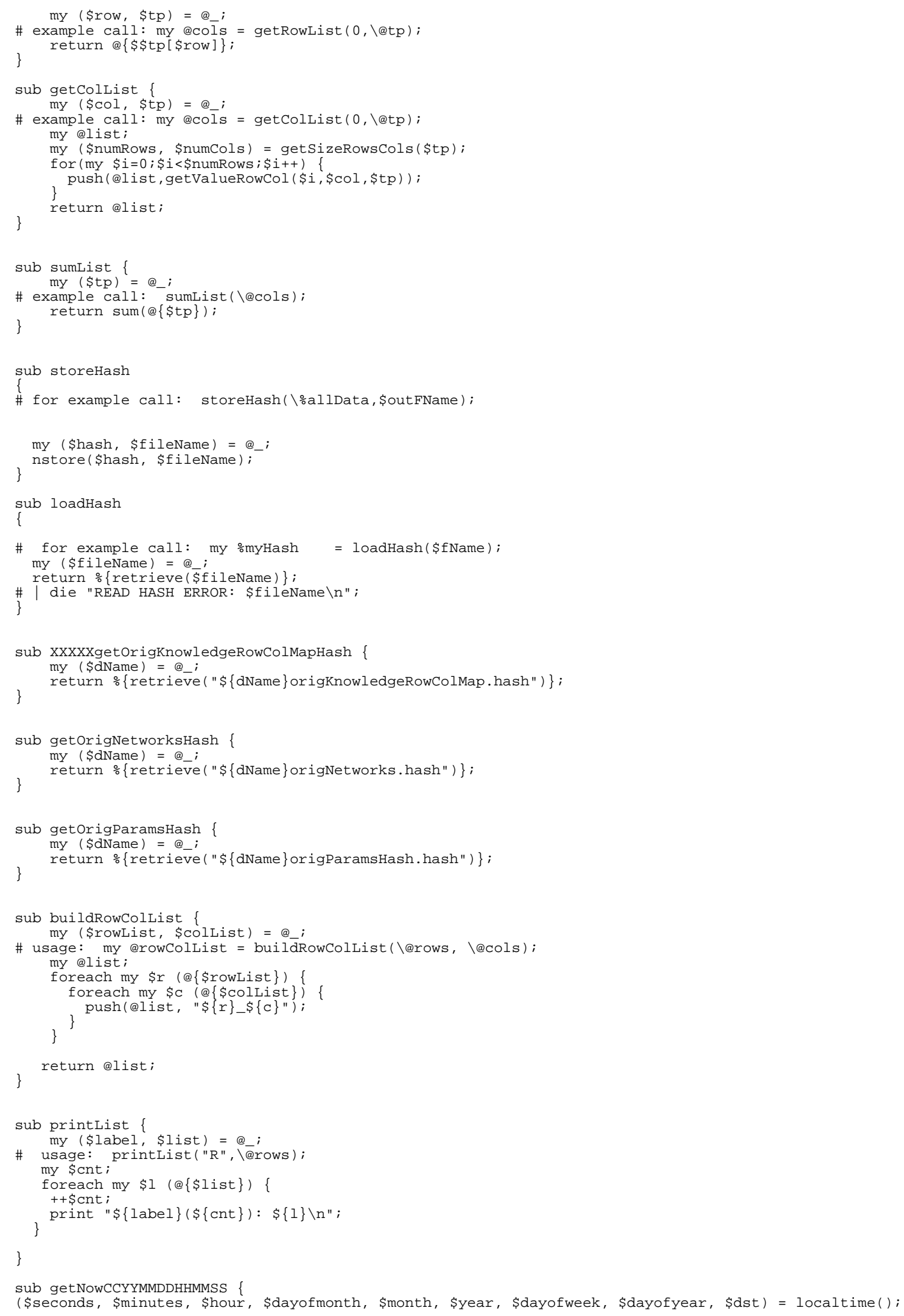

D-29 


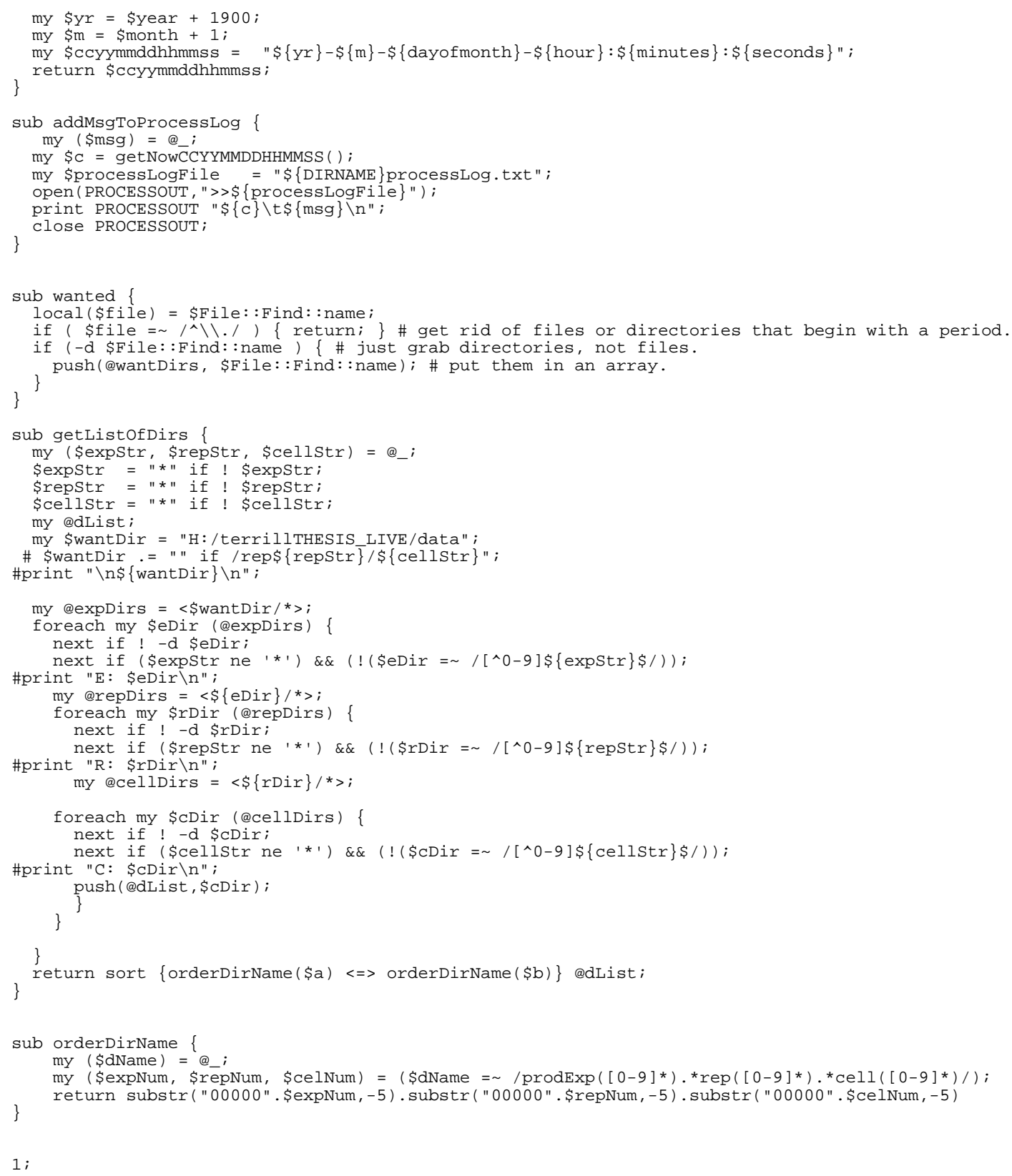

\section{buildRpt.pl}

\#! perl

\# buildRpt.pl

use lib '.';

require '.líib.pl'.

use datastore;

$\begin{array}{ll}\text { my \$rptoutName } & =\text { shift } \\ \text { my \$rptDefId } & =\text { shift } \\ \text { my \$studyExperimentNum } & =\text { shift; } \\ \text { my \$replication } & =\text { shift; } \\ \text { my \$wantCell } & =\text { shift; }\end{array}$




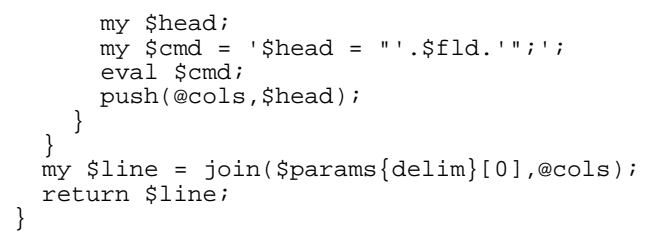

\section{runMultipleGeneric.pl}

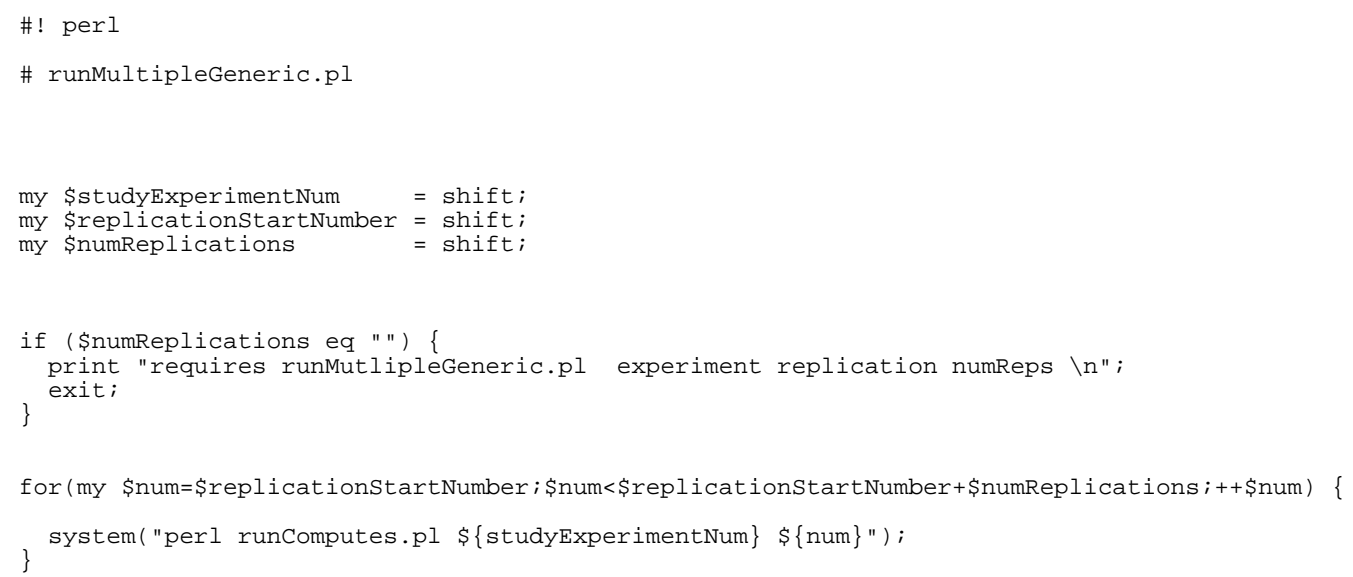

\section{runComputes.pl}

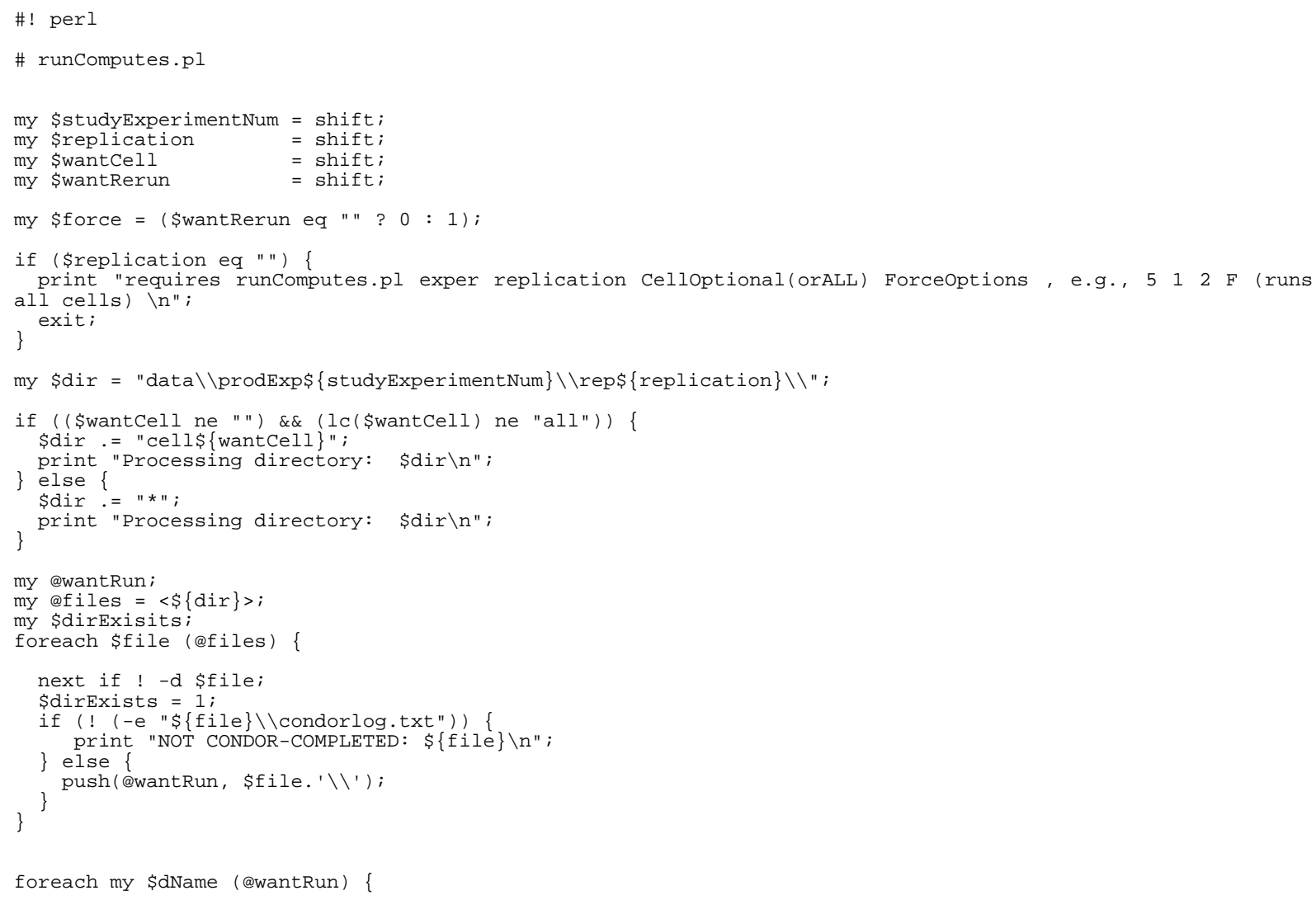


\# $\quad$ print "compute $\$\{$ dName $\} \$\{$ force $\} \backslash n " ;$

\# $\quad$ system("perl./runCompute.pl $\$\{$ dNamé $\$\{$ force $\} ")$

\# $\quad$ system("time /T $2>$ NUL");

print "COMPUTES: $\$\{$ dName $\} \backslash n "$;

\# system("perl ./computesBin/createSmartDataFeedcsV.pl \$\{dName\} \$\{inForce $\} ")$;

\# system("time /T 2>NUL");

system("perl ./computesBin/culturePctAllTpFromDataFeed.pl \$\{dName\} $\$\{$ inForce $\} ")$;

\section{CSV.pm}

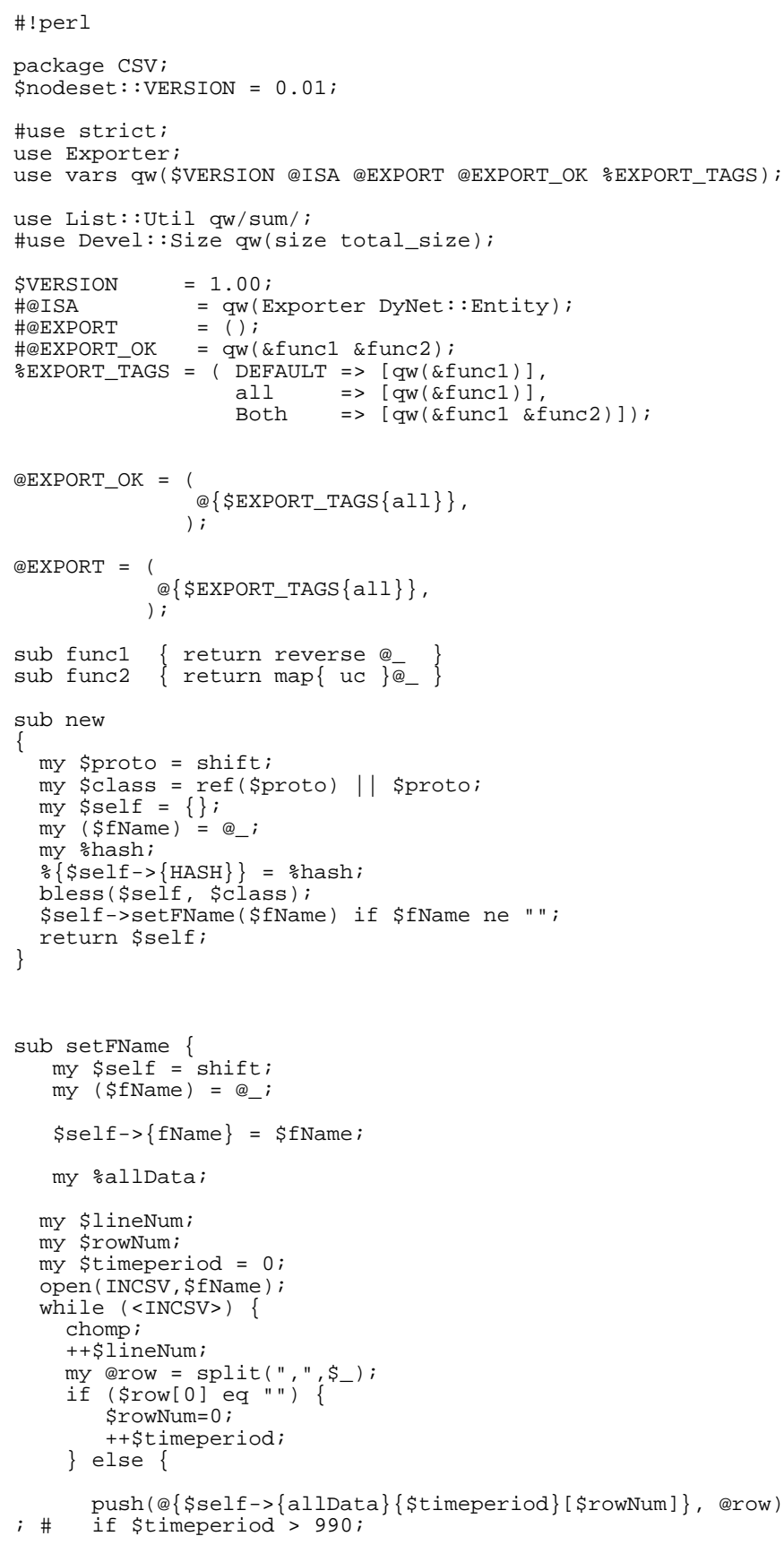

D-33 


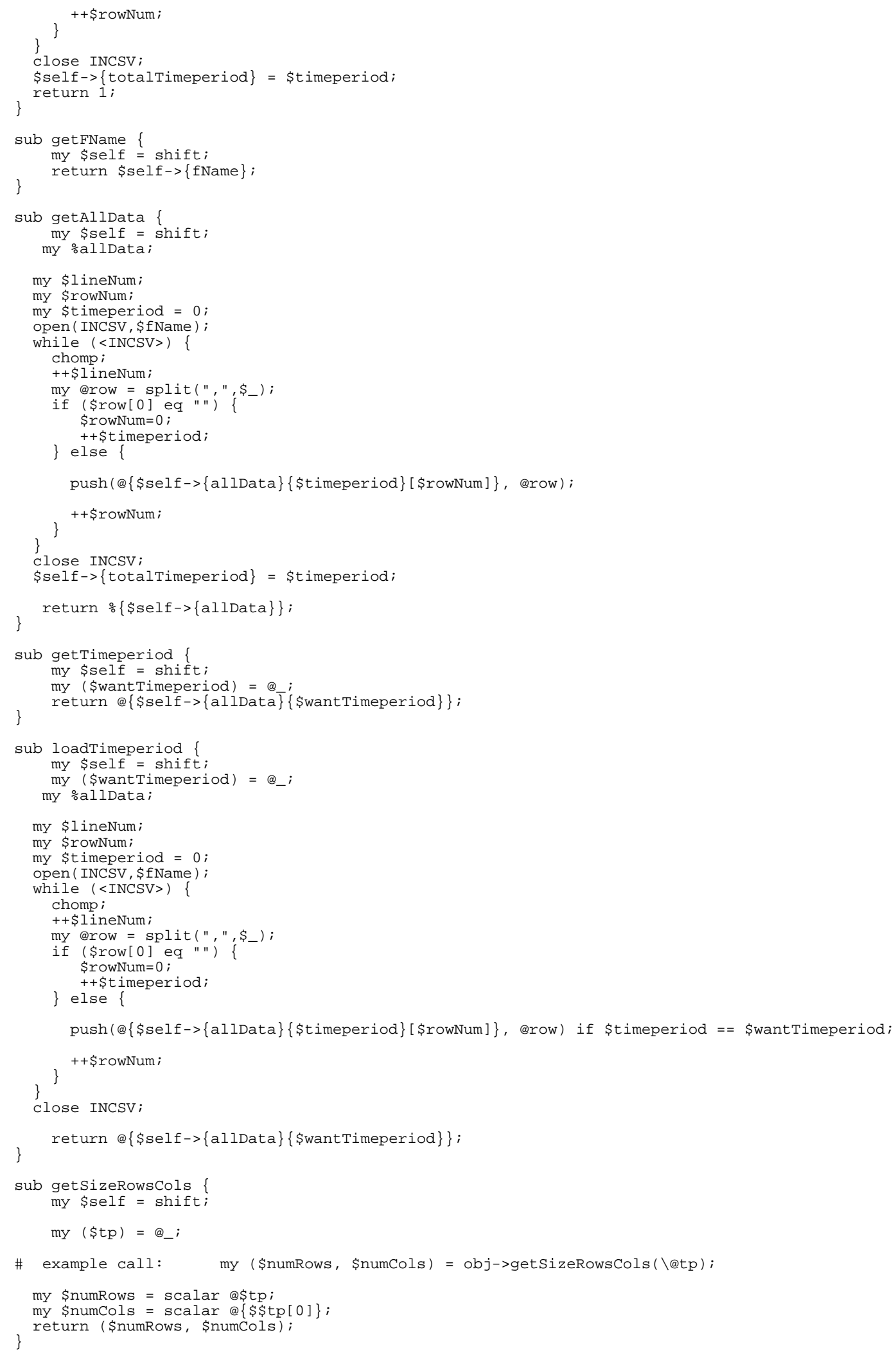

\section{D-34}




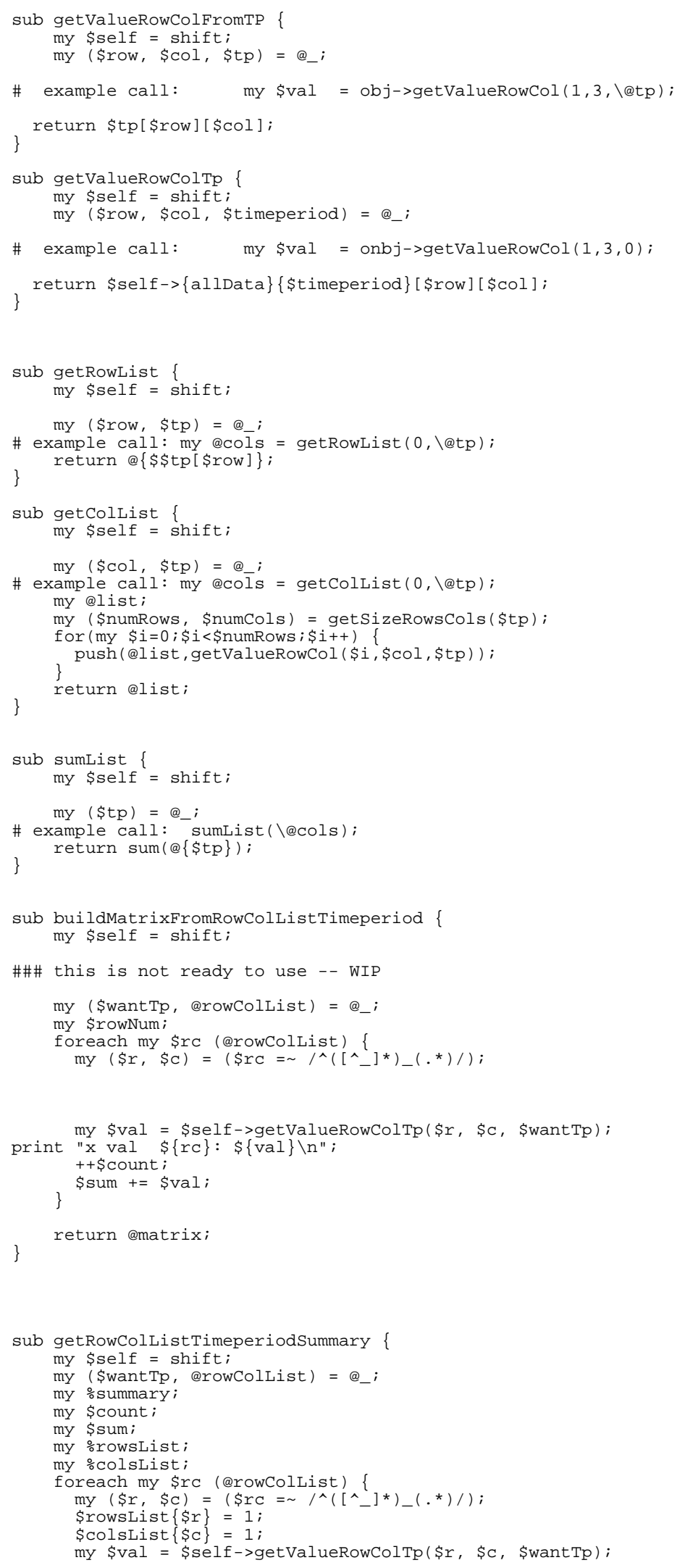

D-35 


$$
\}
$$

return $\$$ tpList $[\$ r o w][\$ \operatorname{col}]$

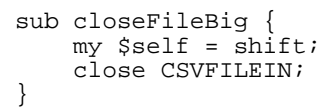

$1 ;$

\section{kNetwork.pm}

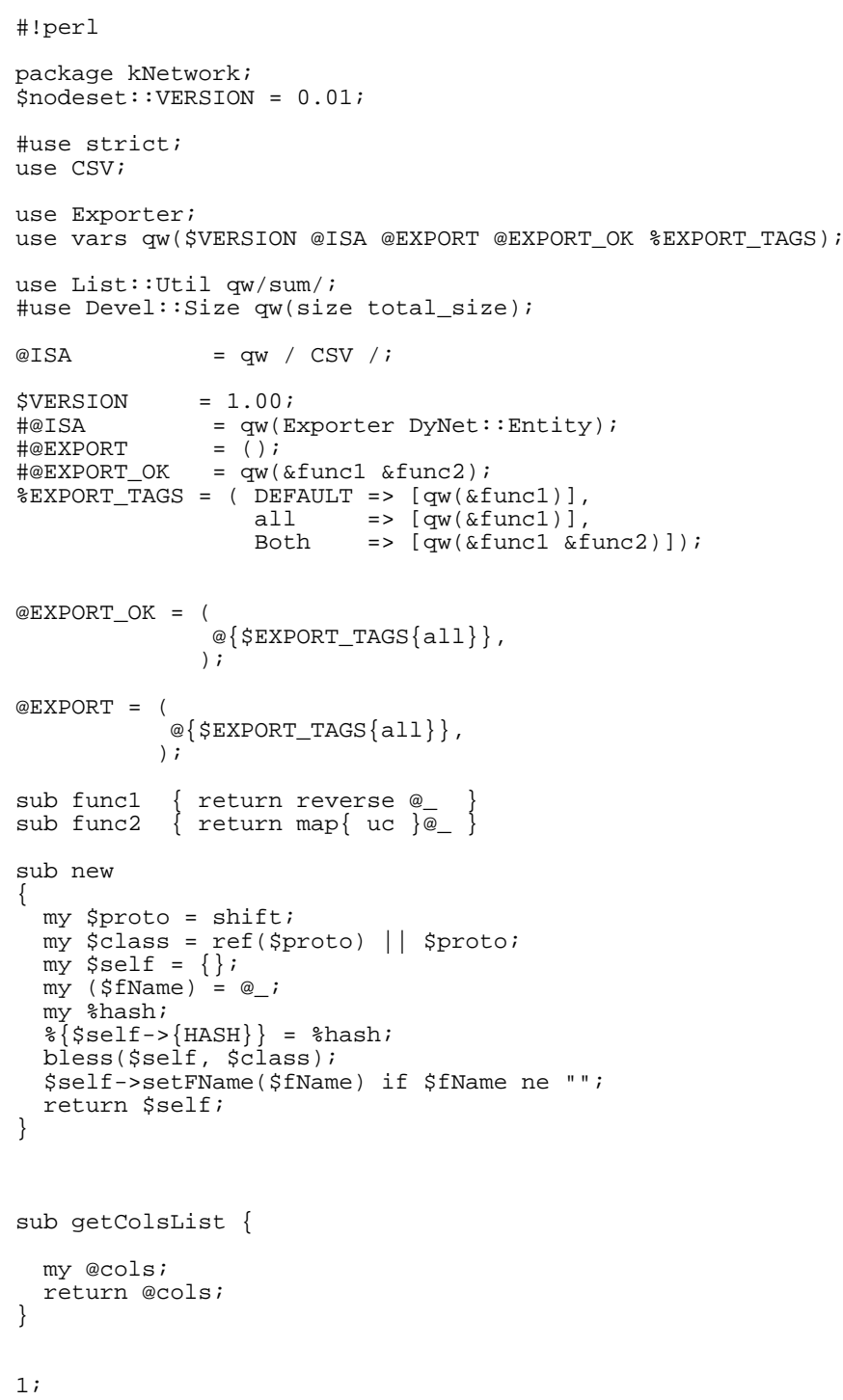

\section{knowledgeRowColMap.pm}




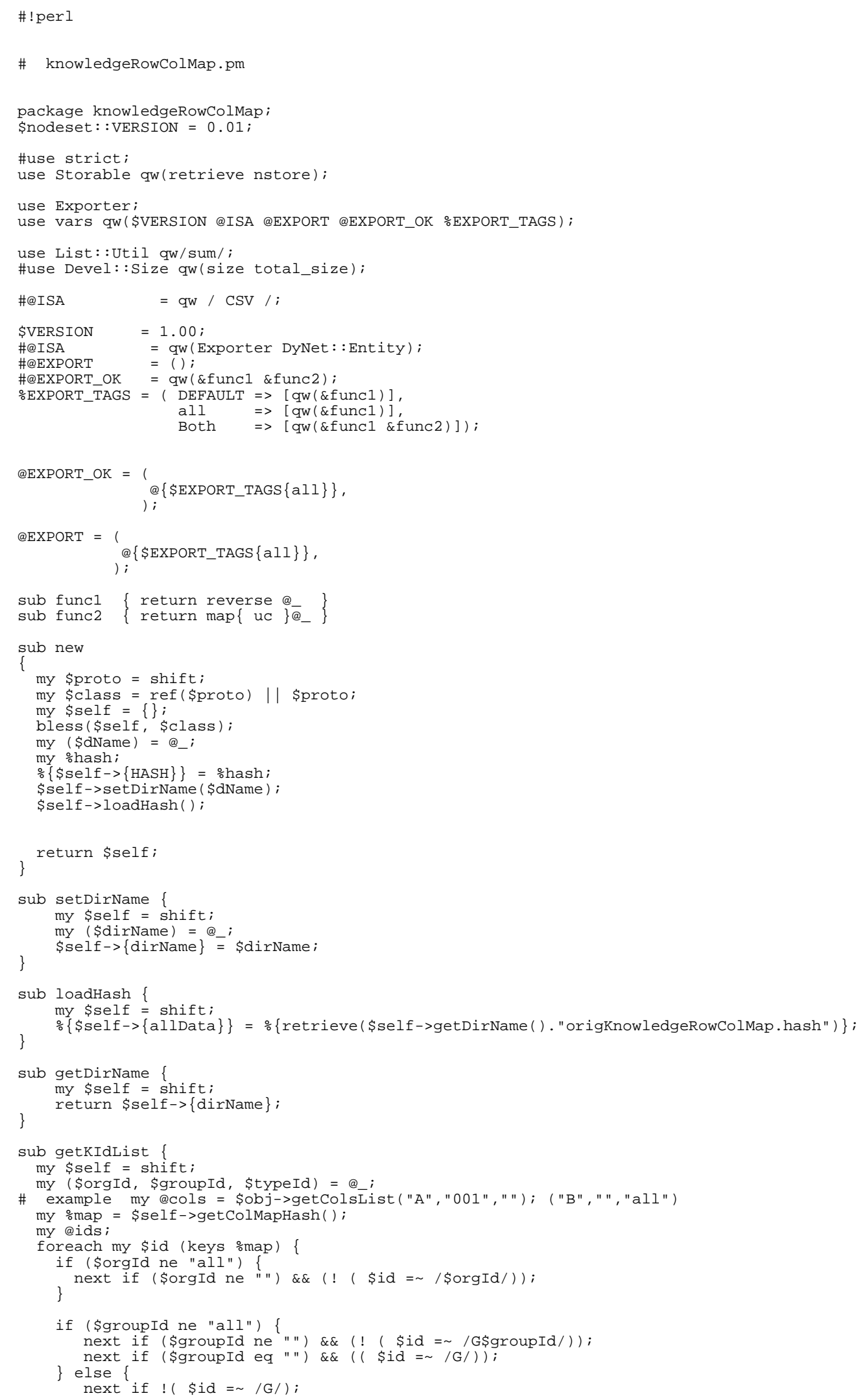

D-38 


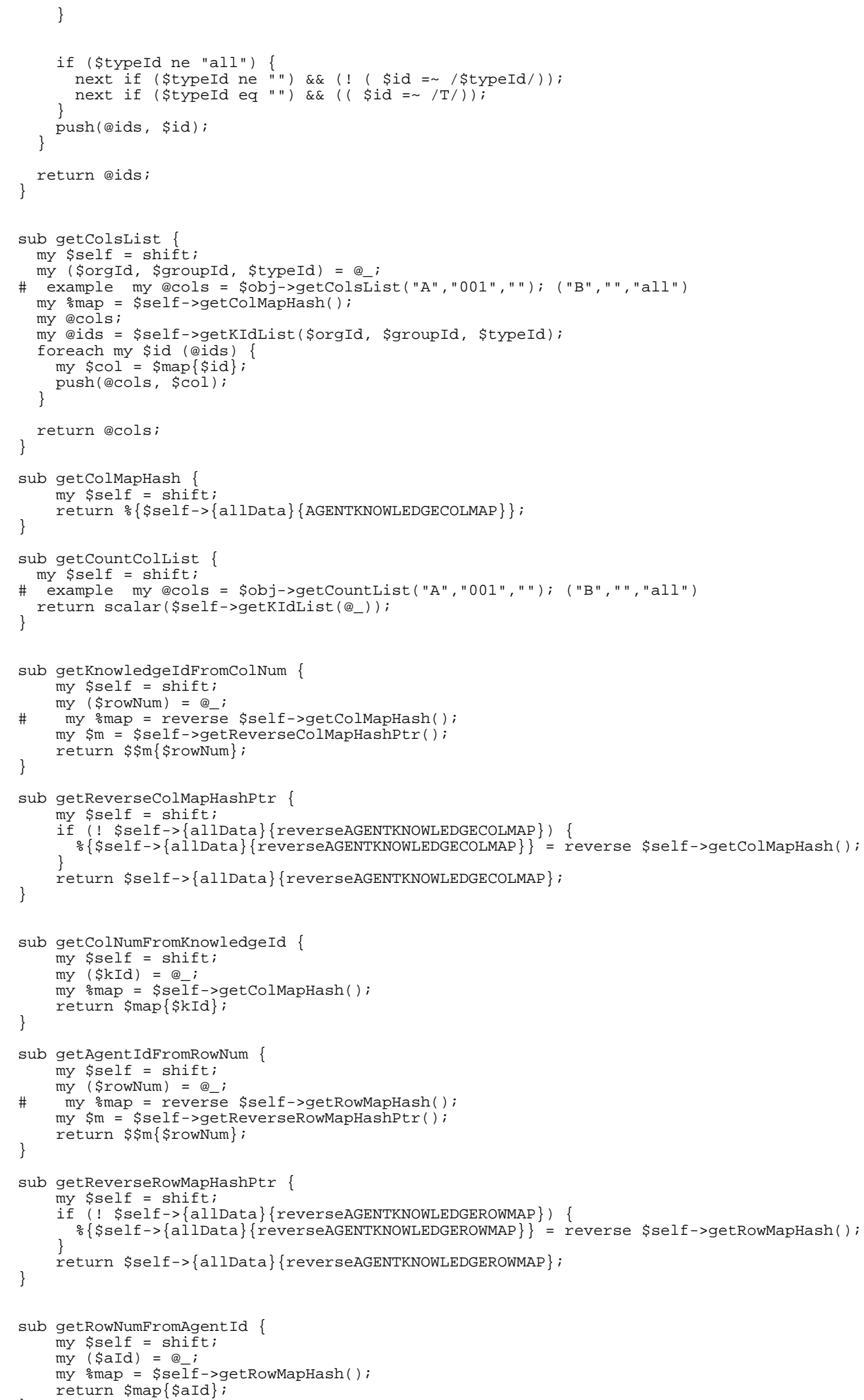




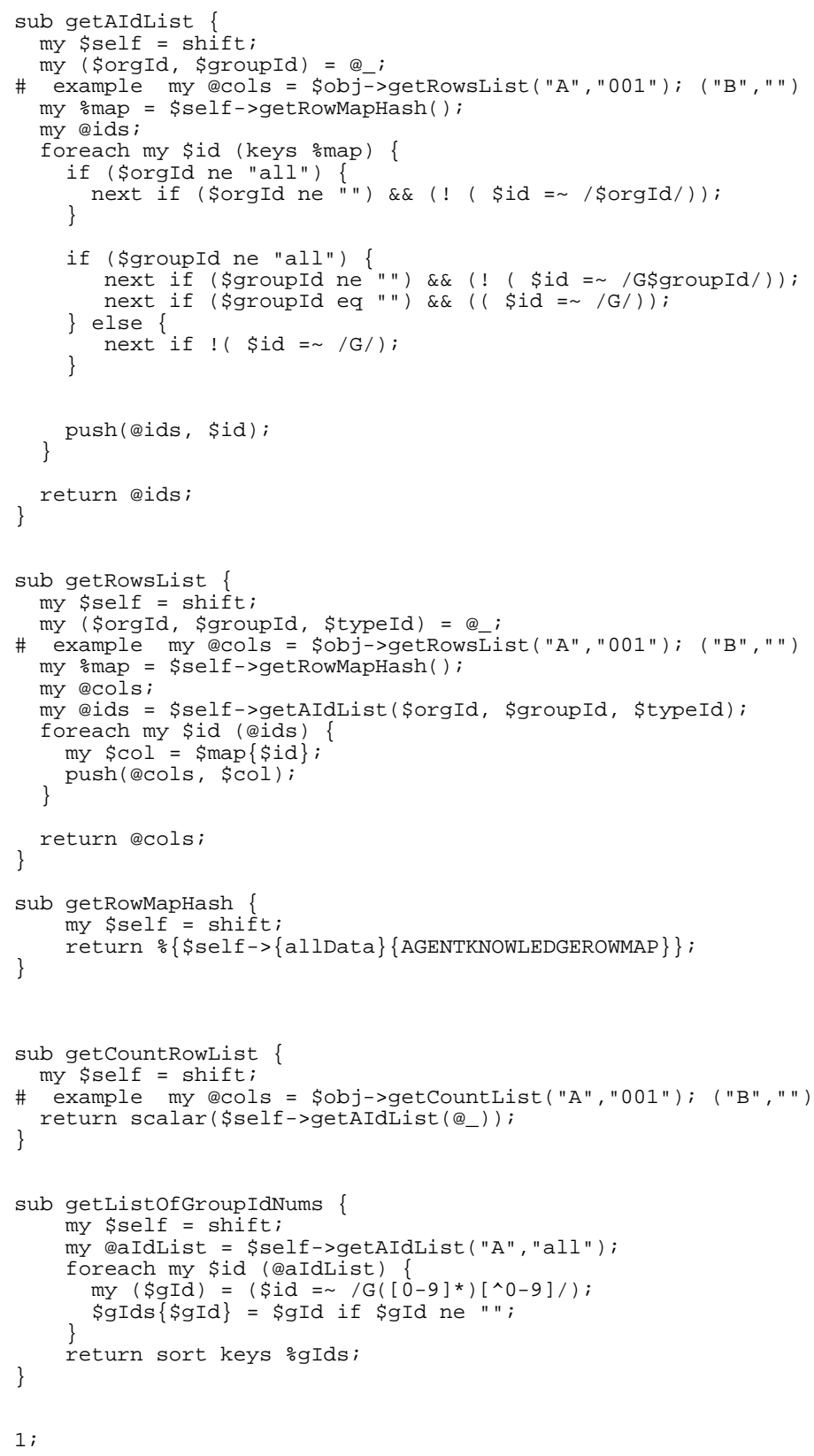

\section{dataFeed.pm}

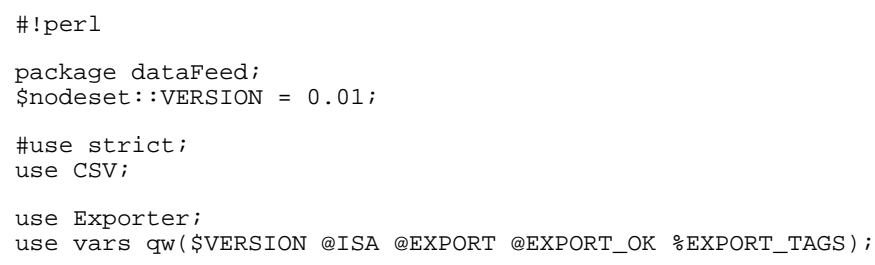




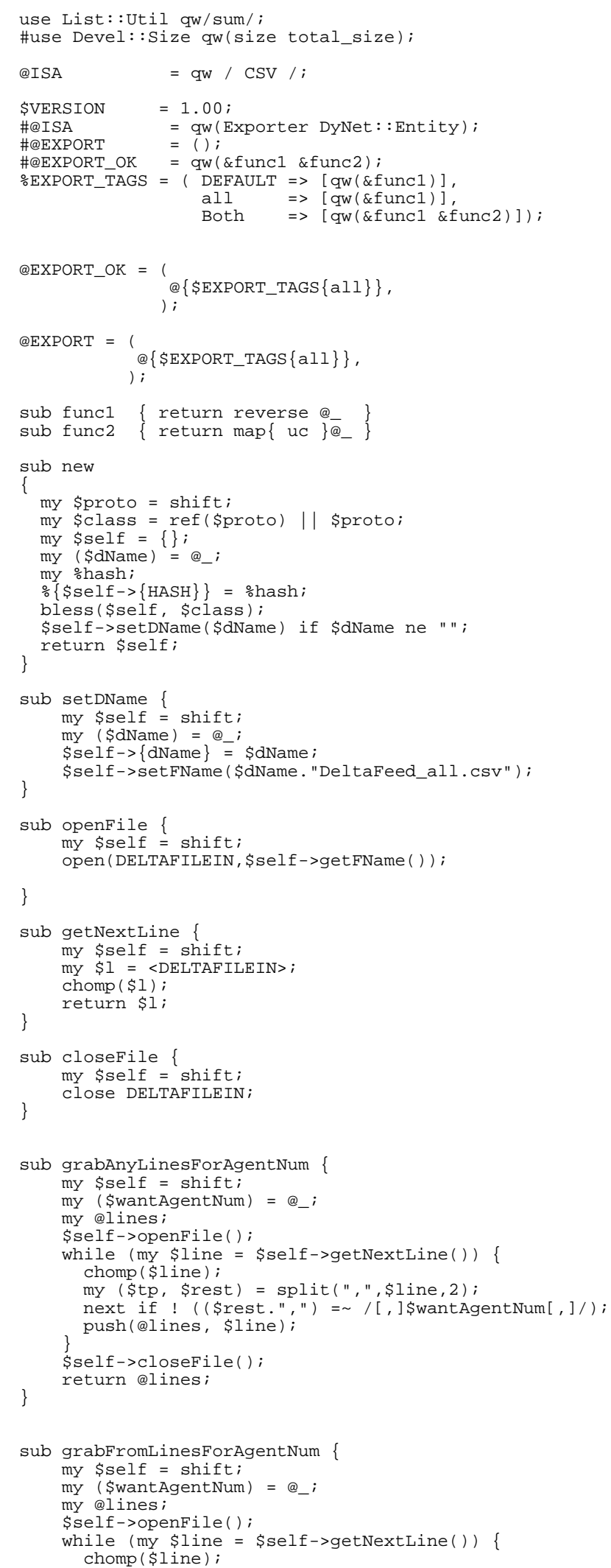




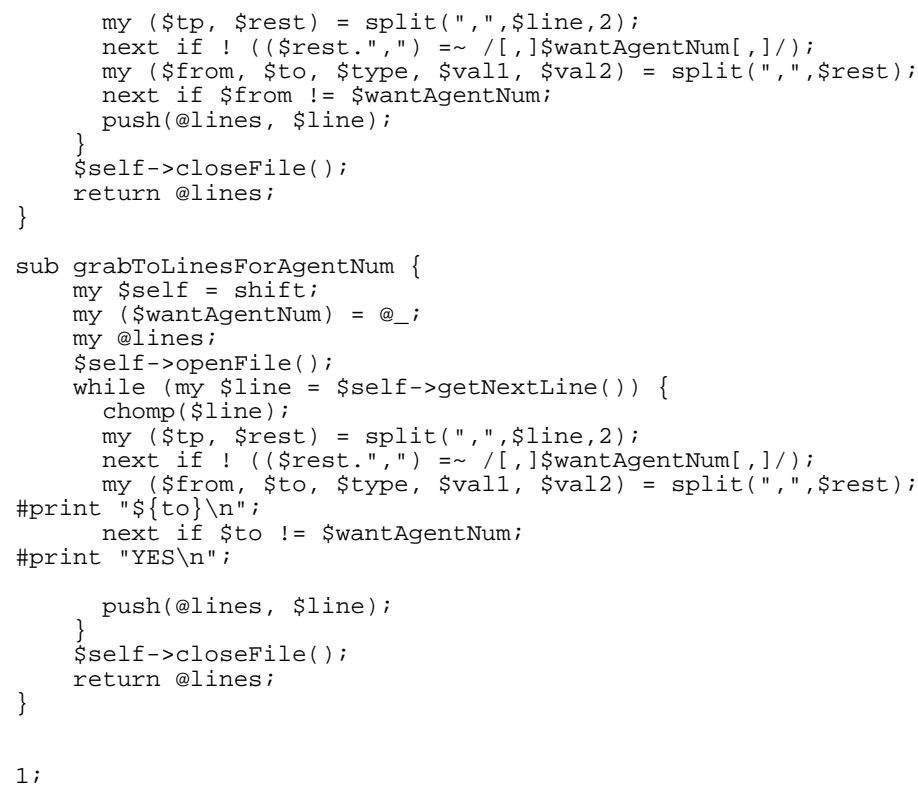

\section{smartDataFeed.pm}

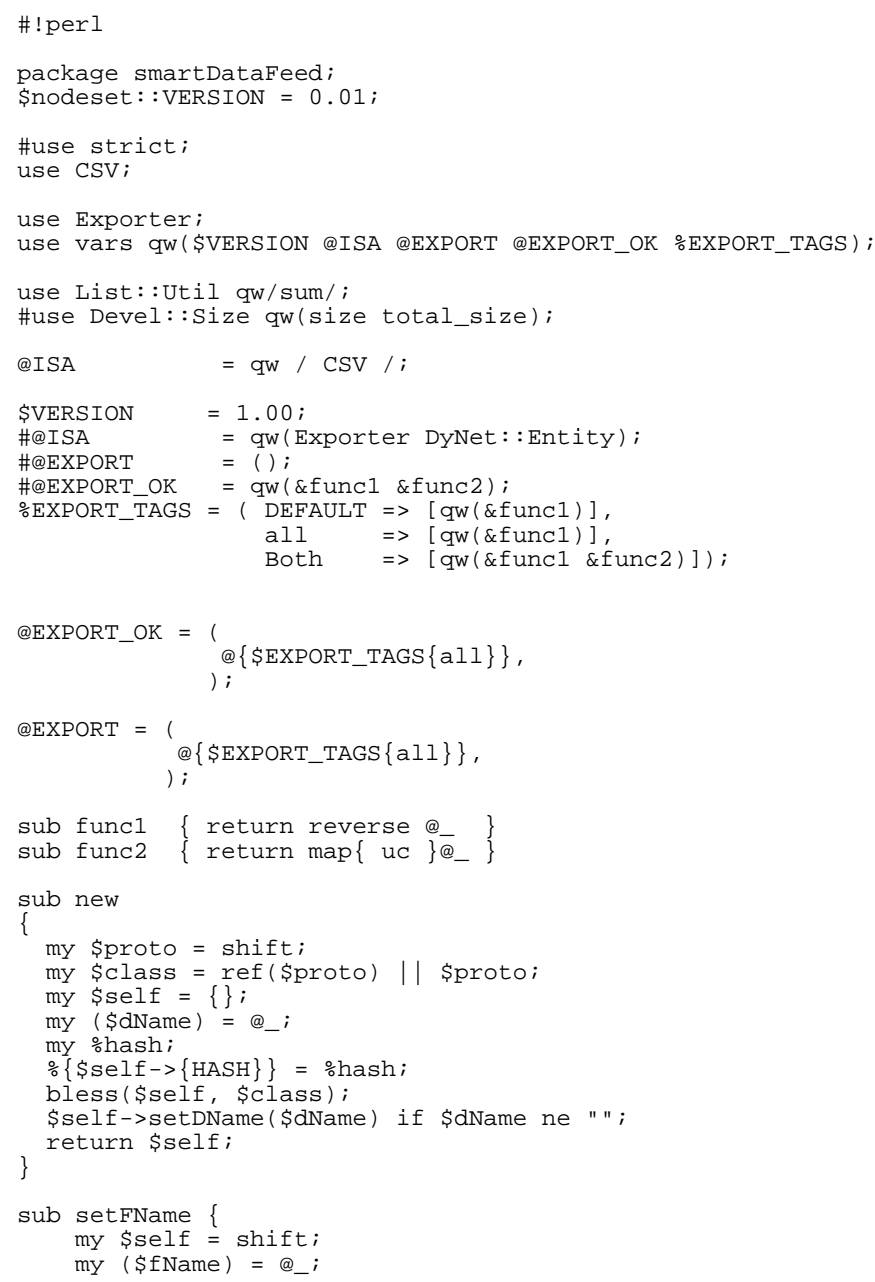




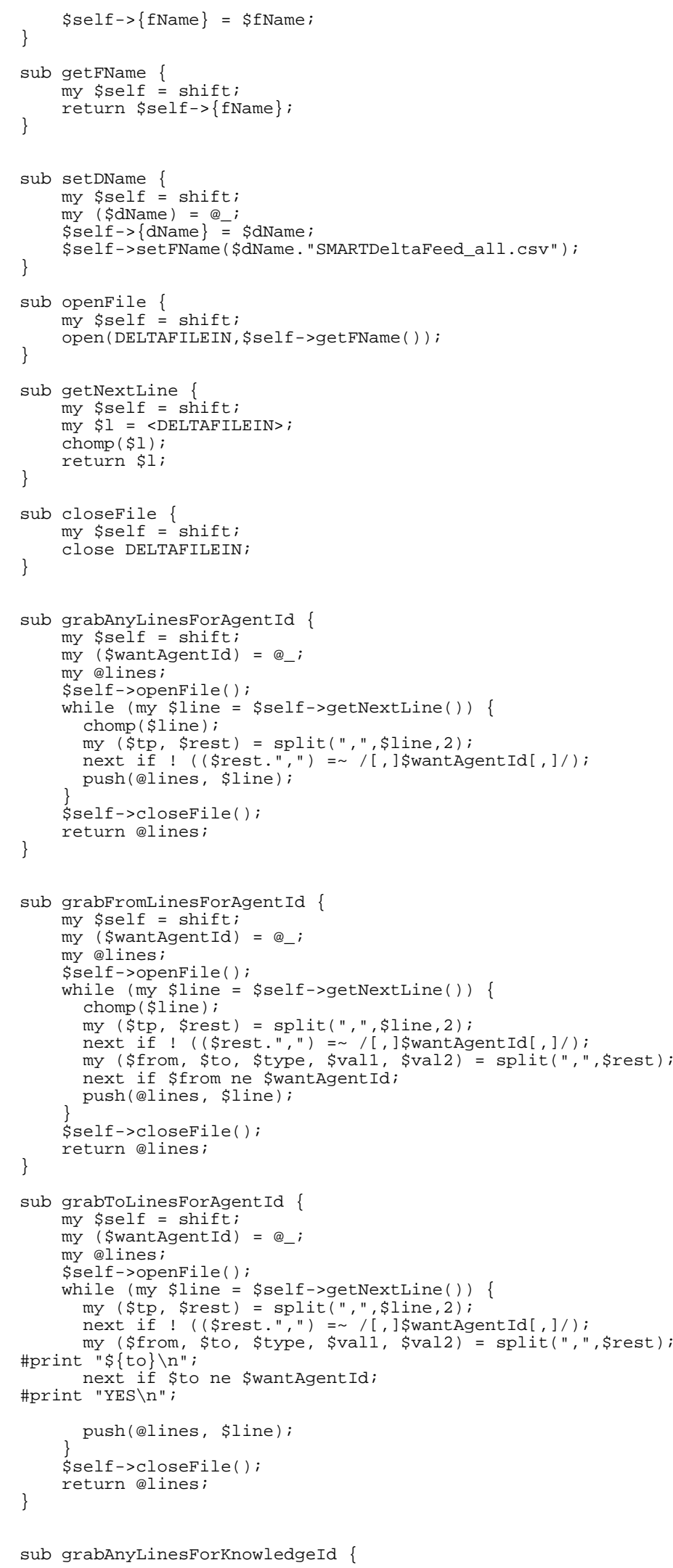

sub grabAnyLinesForKnowledgeId \{ 


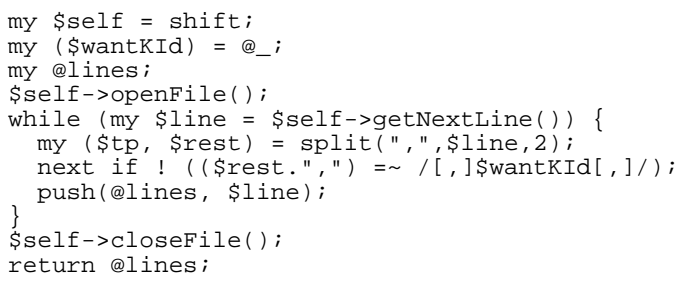

\section{dataStore.pm}

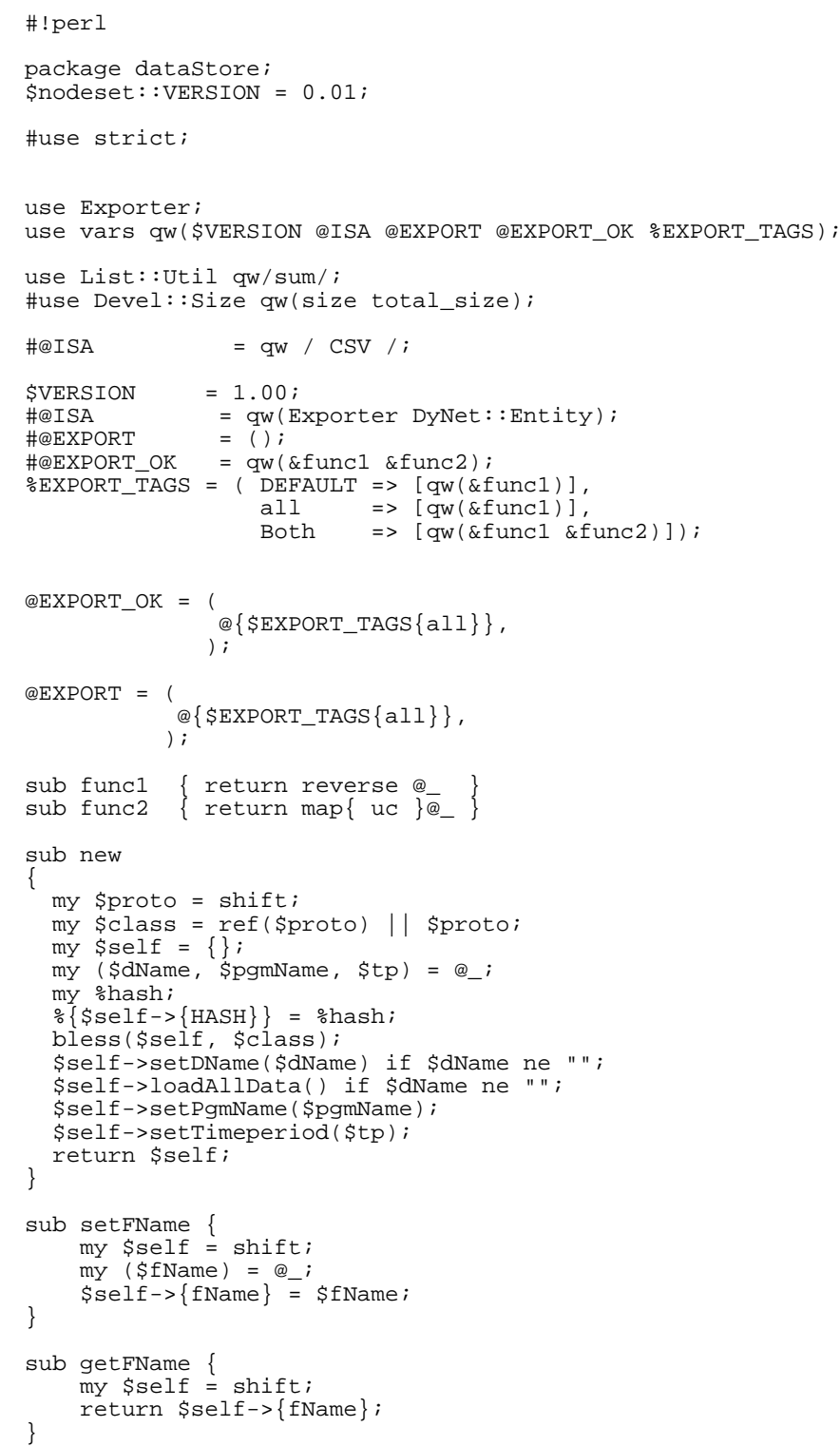




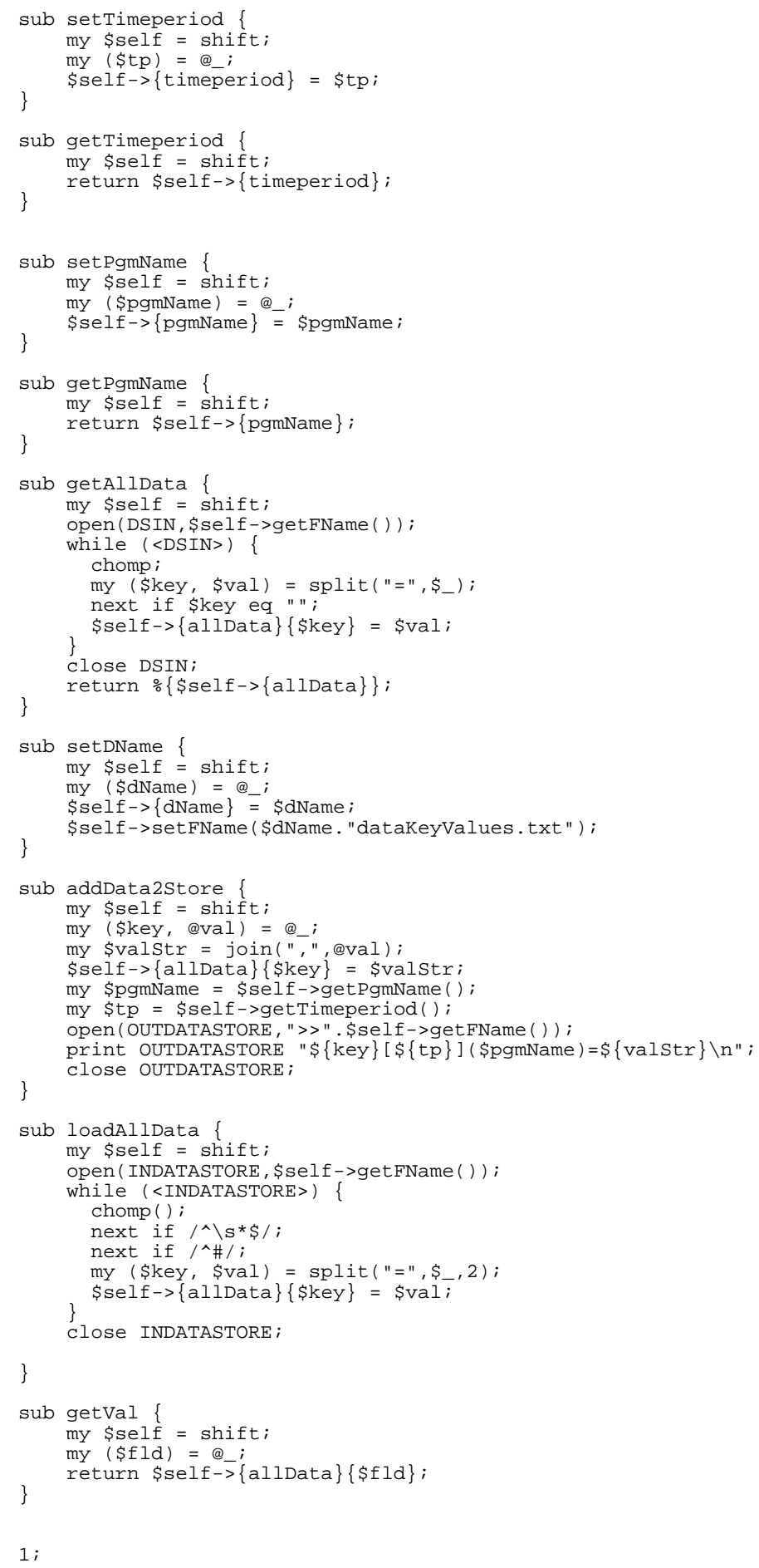




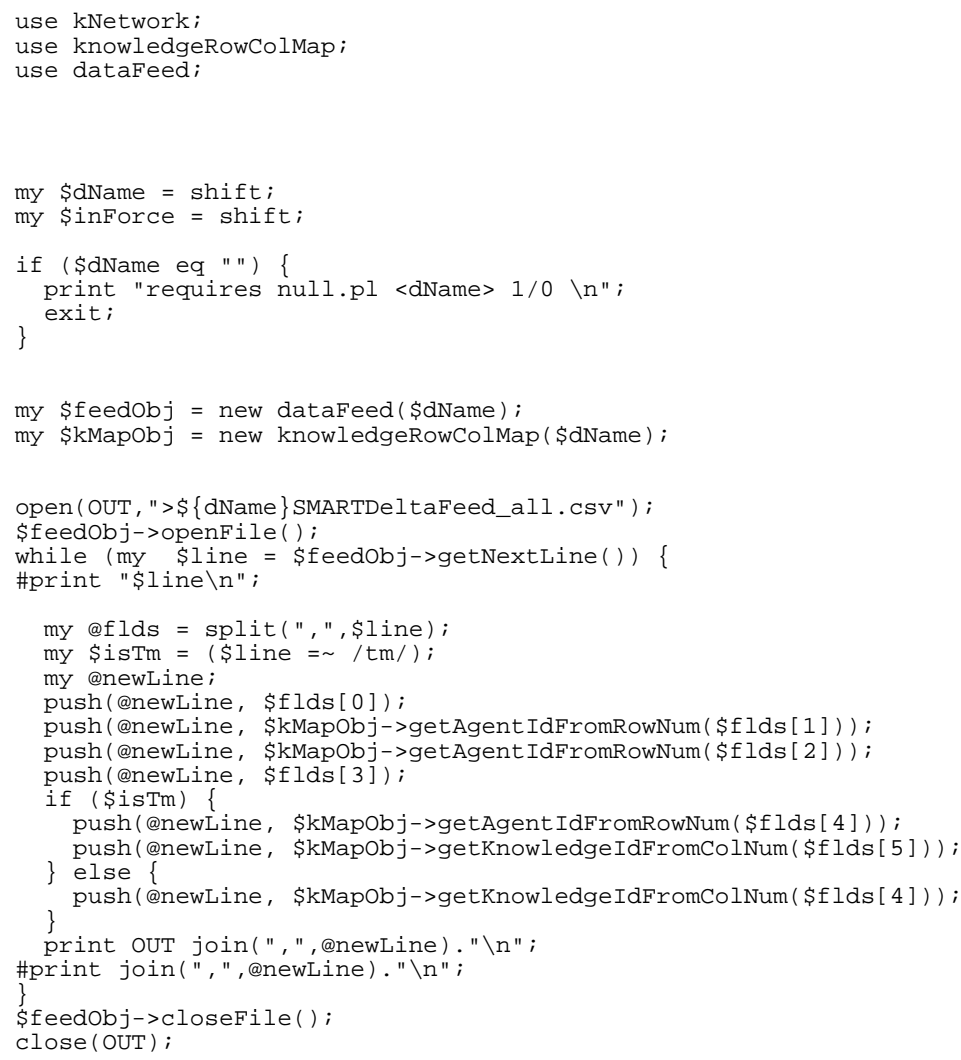

\section{exit;}

\section{computeBin/culturePctAllTpFromDataFeed.pl}

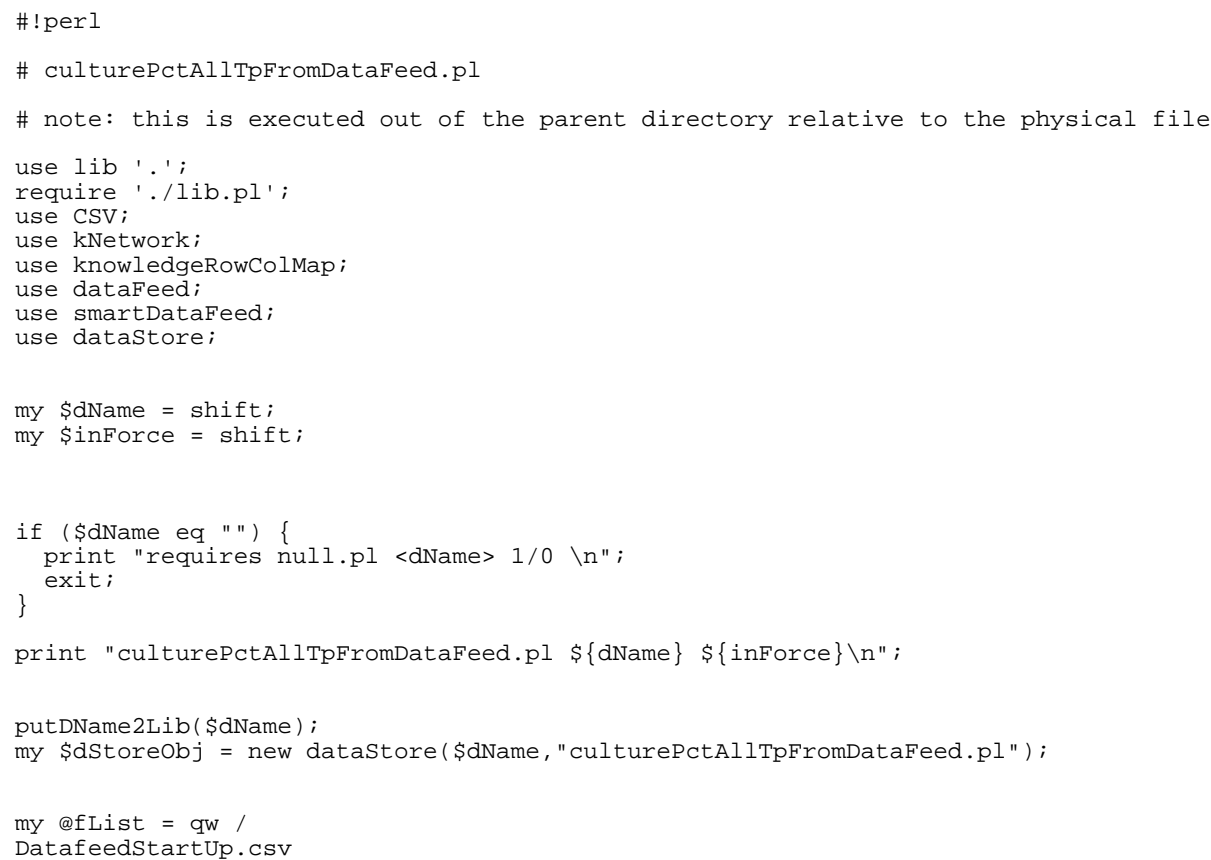




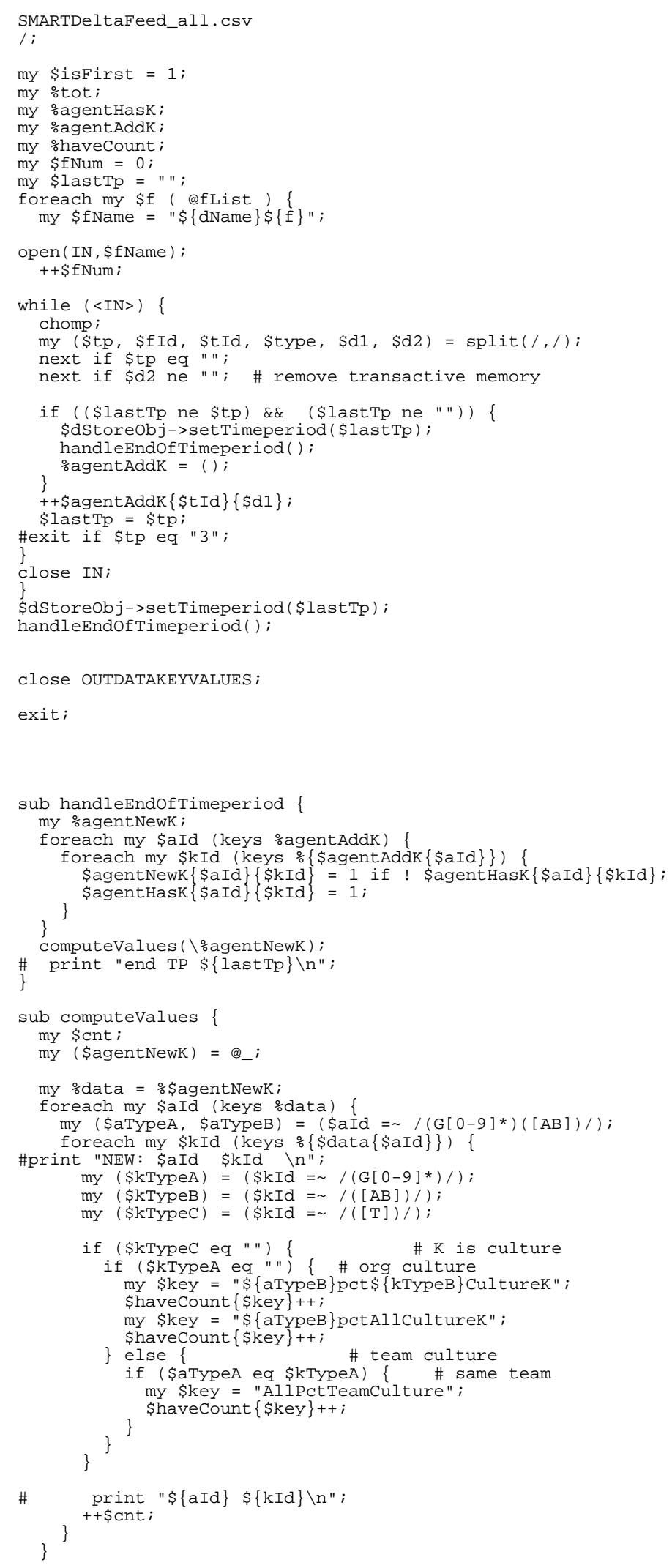




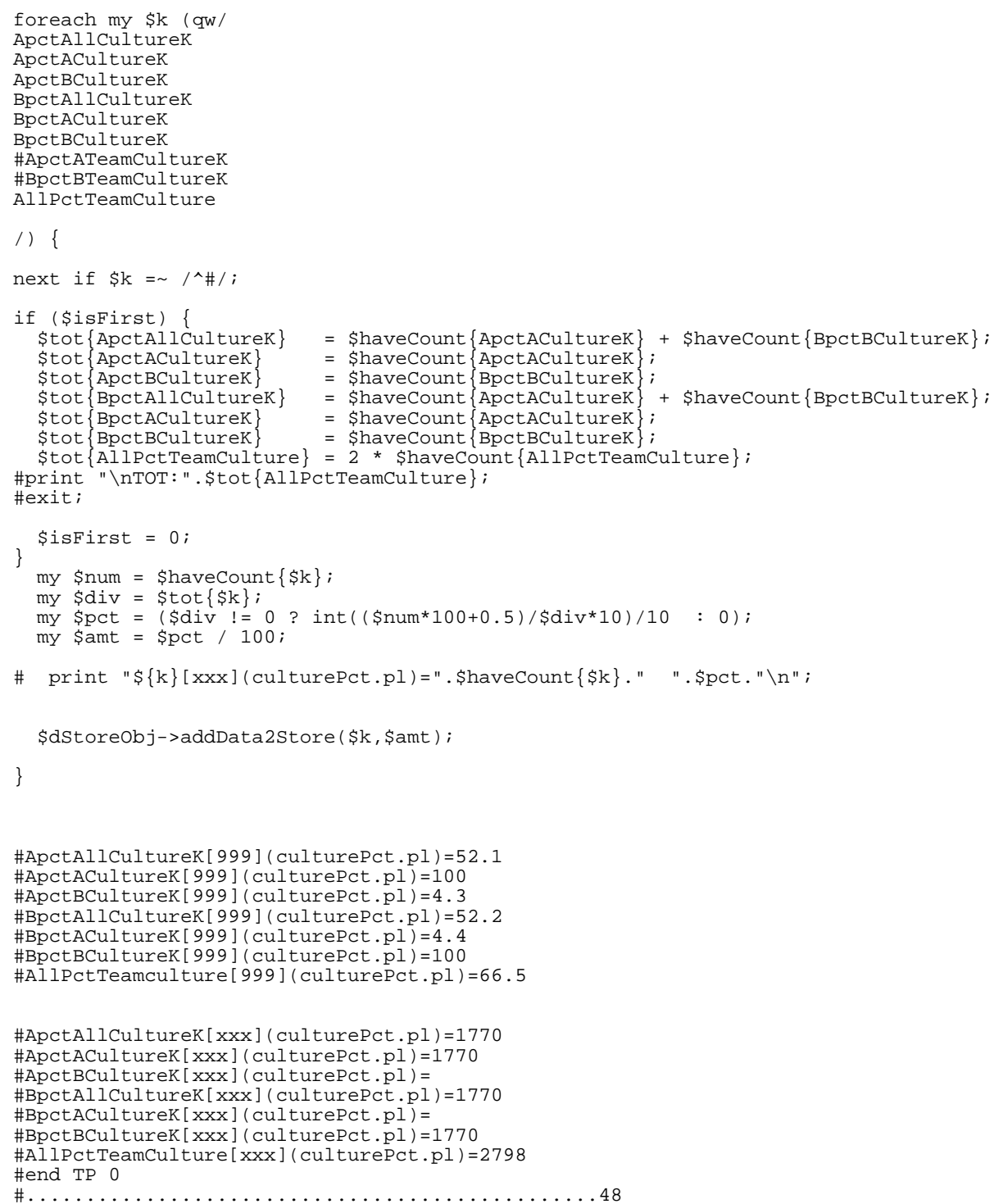

\section{computesBin/ culturePct.pl}

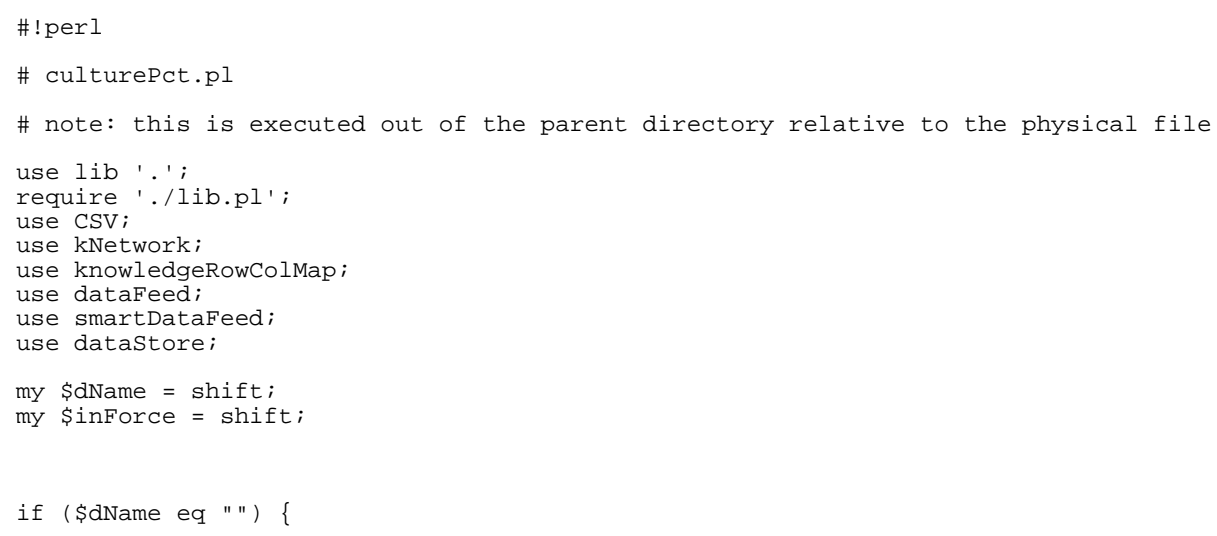




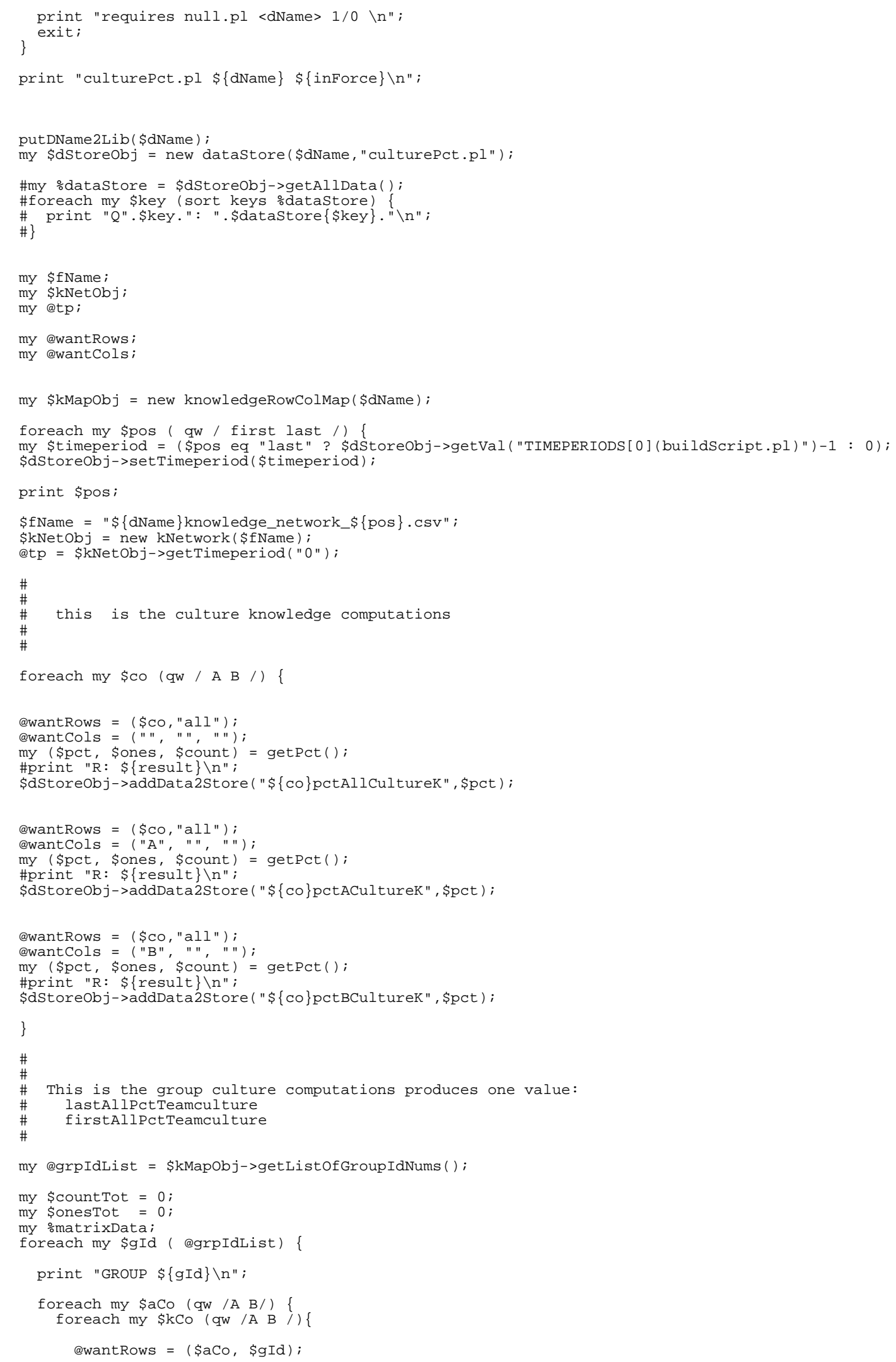




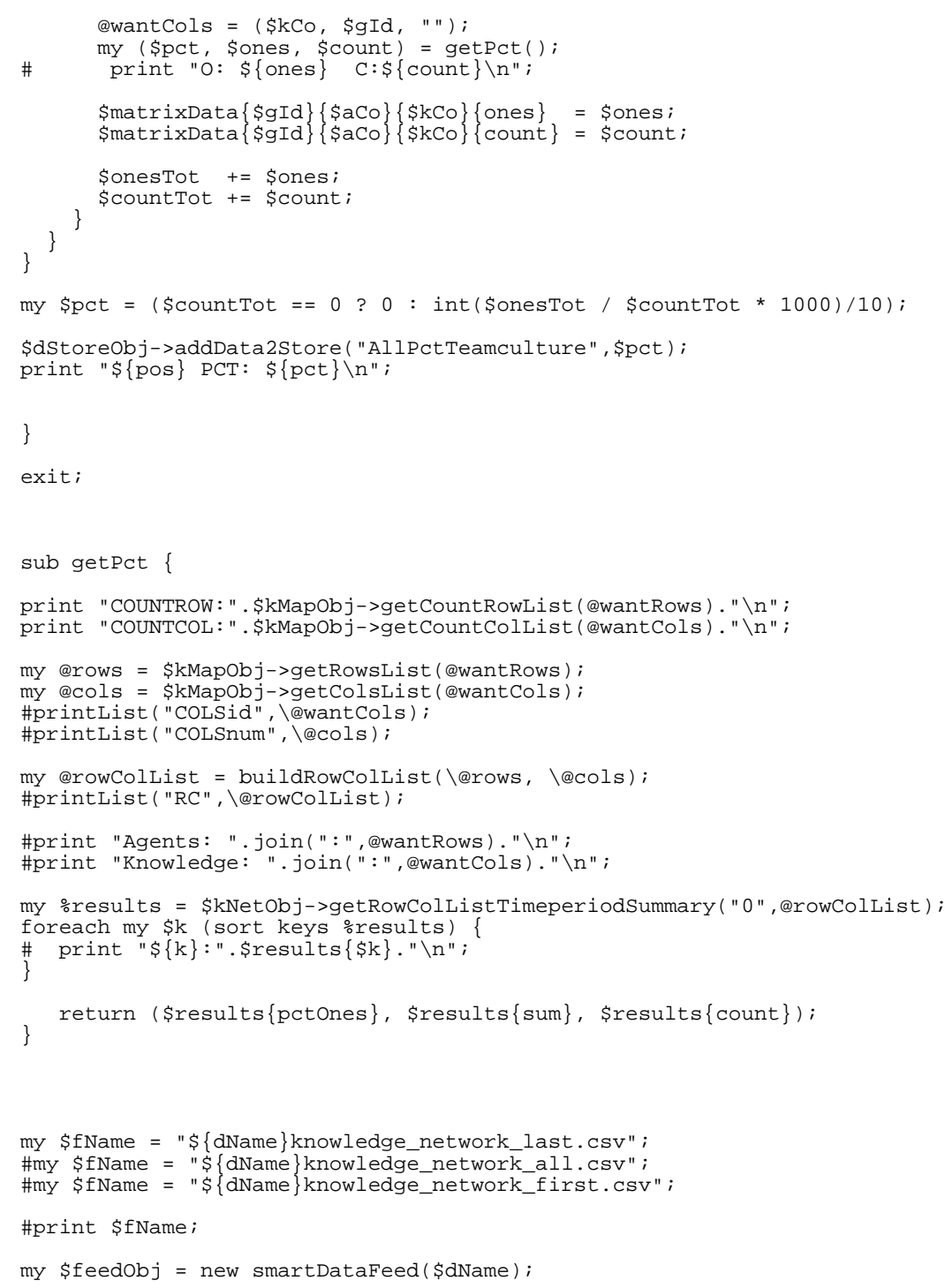




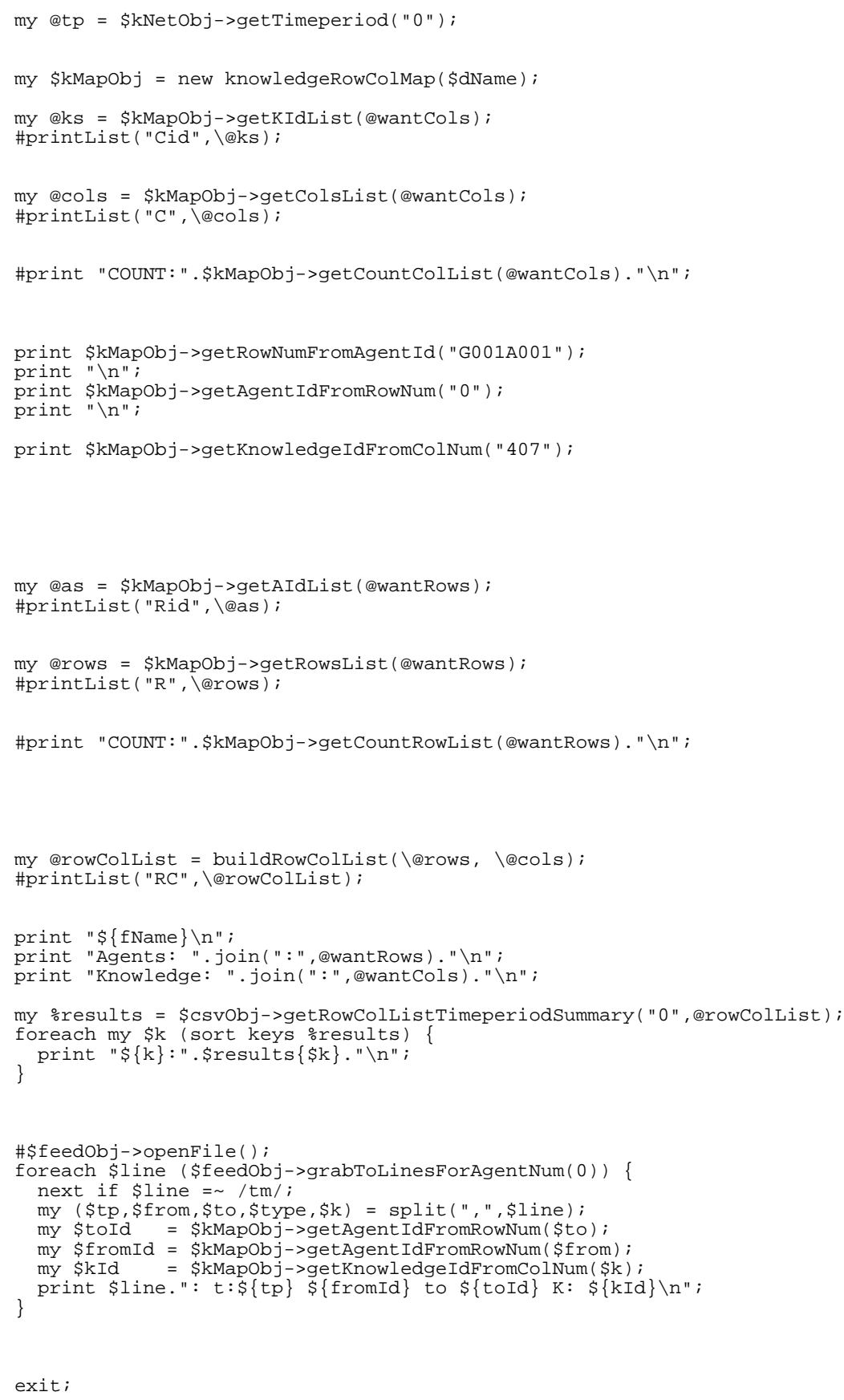

\section{computesBin/ culturePctAlltp.pl}

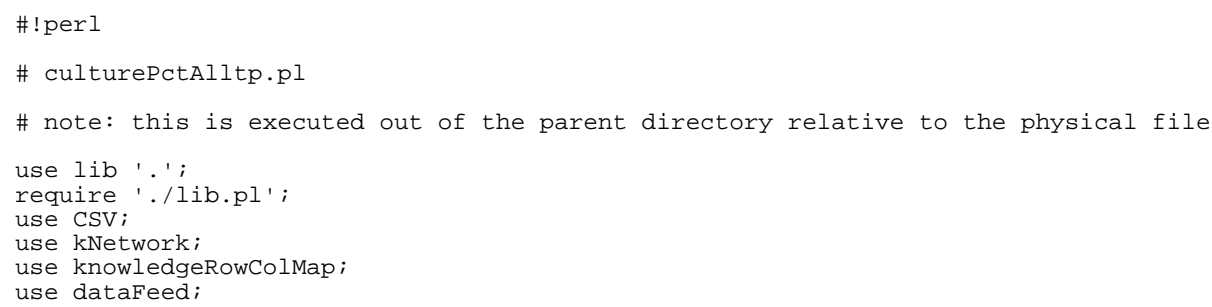




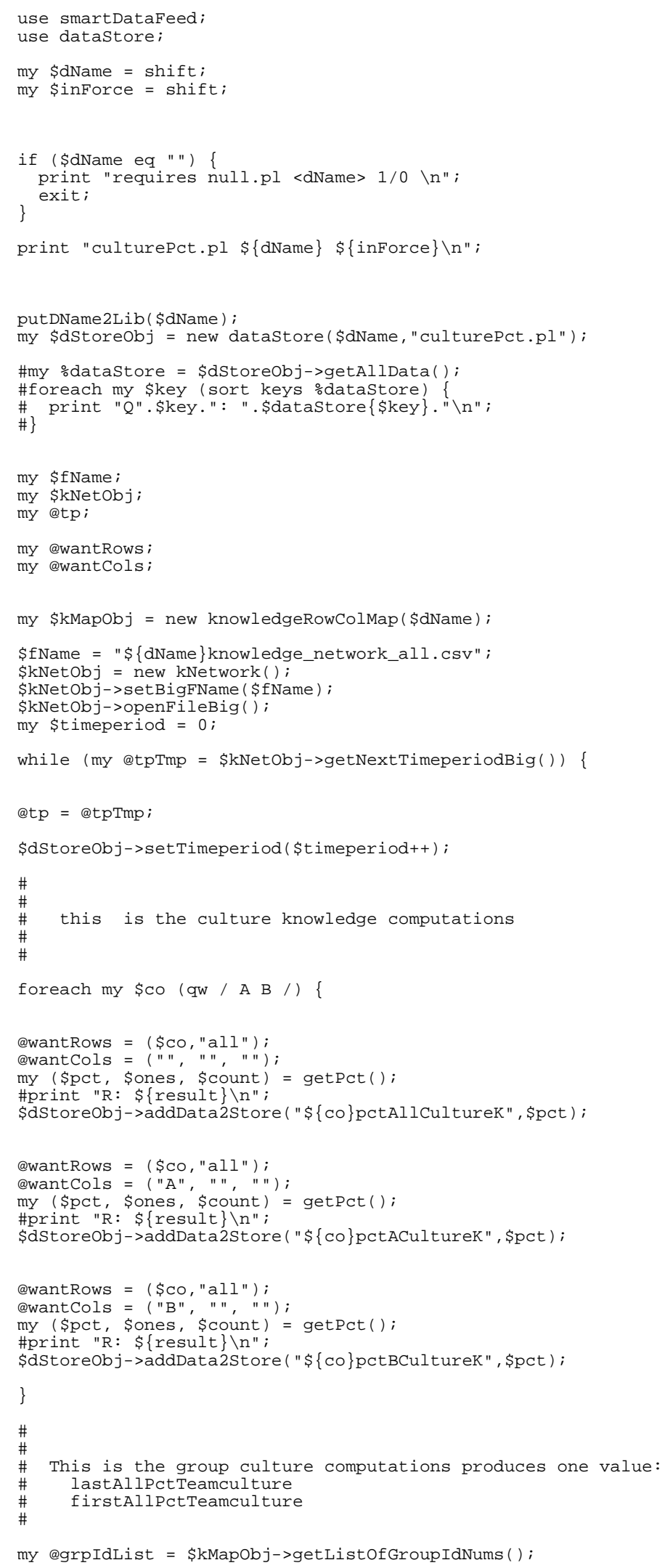




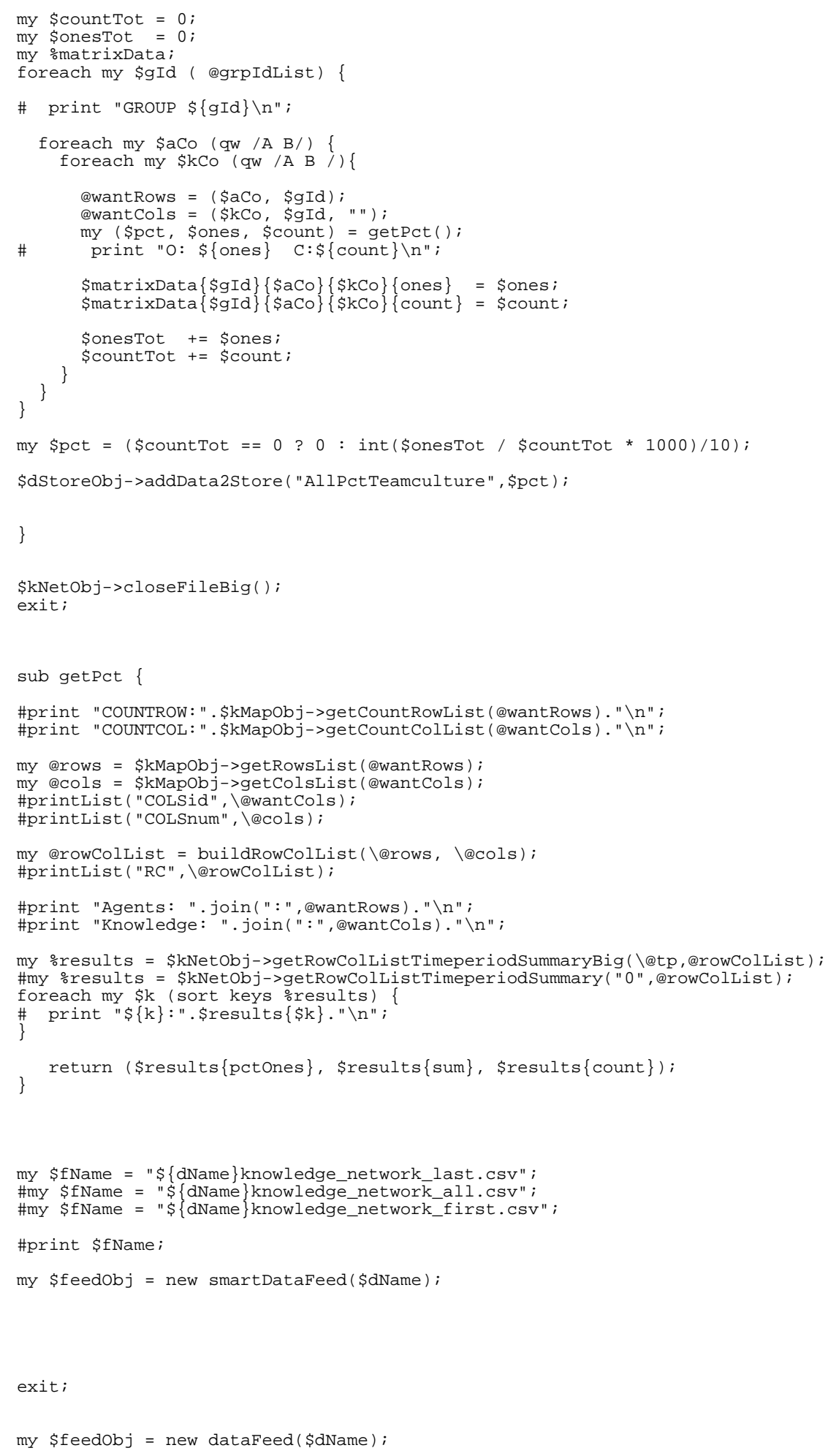

my $\$$ fName $=" \$\{$ dName $\}$ knowledge_network_last.csv"

\#my $\$$ fName = "\$\{dName $\}$ knowledge_network_all.csv";

\#my $\$$ fName = "\$\{dName $\}$ knowledge_network_first.csv";

\#print \$fName;

my $\$$ feedobj = new smartDataFeed $(\$ d$ Name $)$;

exit;

my $\$$ feedobj $=$ new dataFeed $(\$ d$ Name $)$;

my @wantRows:

\section{D-53}




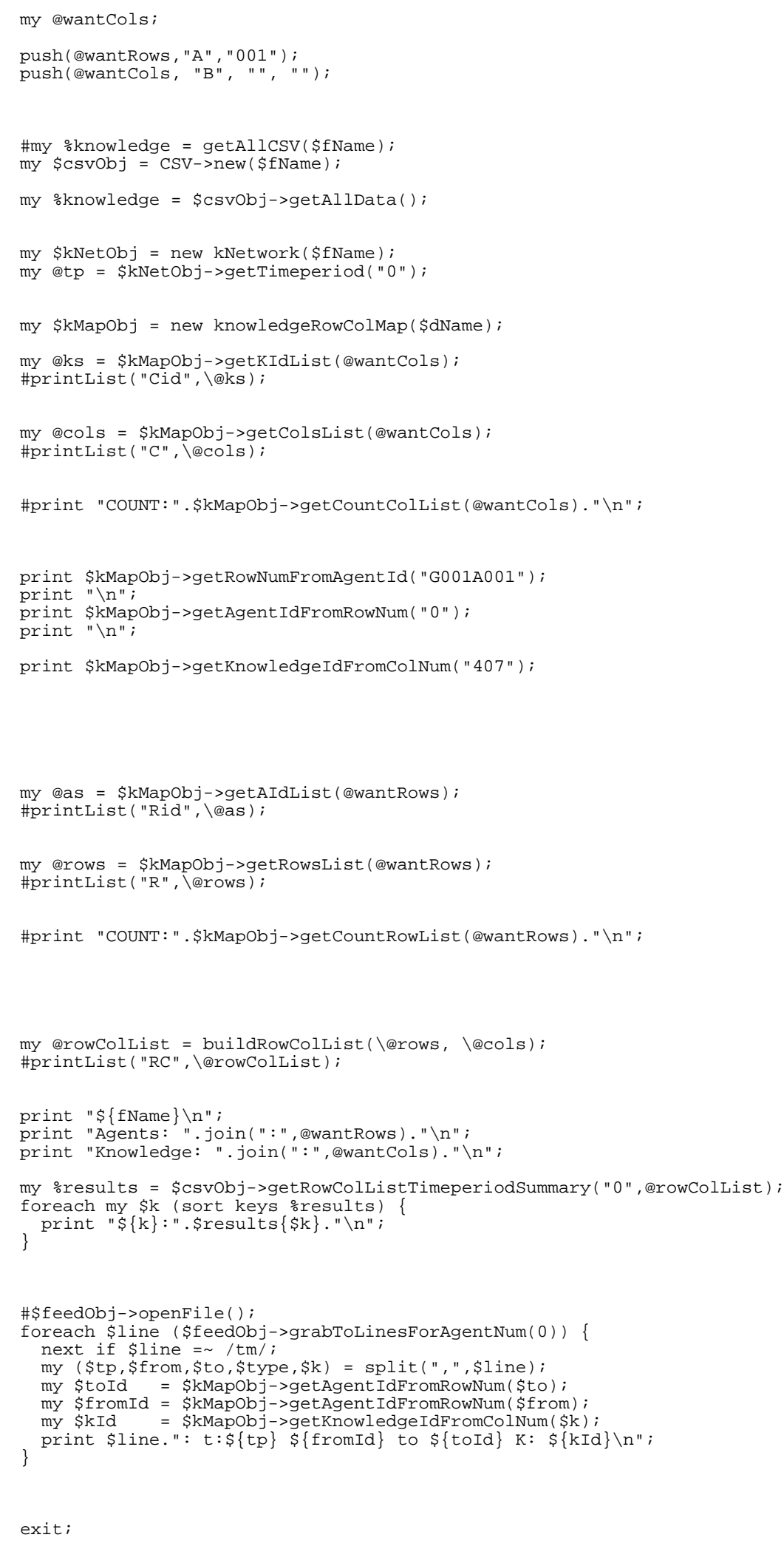


(BLANK)

D-55 
(END)

D-56 